\title{
LA-UR-12-24571
}

Approved for public release; distribution is unlimited.

Title:

Author(s):
P24 Plasma Physics Summer School 2012 Los Alamos National Laboratory Summer lecture series for students

Intended for:

Intrator, Thomas $\mathrm{P}$.

Bauer, Bruno

Fernandez, Juan C.

Daughton, William S.

Flippo, Kirk A.

Weber, Thomas

Awe, Thomas J.

Kim, Yong Ho

P24 Plasma Physics Summer School

Web

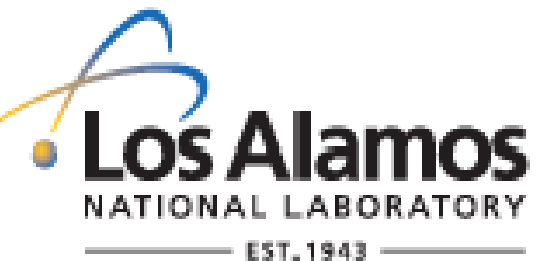

Disclaimer:

Los Alamos National Laboratory, an affirmative action/equal opportunity employer,is operated by the Los Alamos National

Security, LLC for the National NuclearSecurity Administration of the U.S. Department of Energy under contract DE-AC52-06NA25396. By approving this article, the publisher recognizes that the U.S. Government retains nonexclusive, royalty-free license to publish or reproduce the published form of this contribution, or to allow others to do so, for U.S. Government purposes.

Los Alamos National Laboratory requests that the publisher identify this article as work performed under the auspices of the

U.S. Departmentof Energy. Los Alamos National Laboratory strongly supports academic freedom and a researcher's right to publish; as an institution, however, the Laboratory does not endorse the viewpoint of a publication or guarantee its technical correctness. 


\section{P24 Plasma Physics Summer School 2012 \\ Los Alamos National Laboratory Summer lecture series for students}

1. Tom Intrator, P24 LANL: Kick off, Introduction - What is a plasma?

2. Bruno Bauer, Univ. Nevada-Reno: Derivation of plasma fluid equations

3. Juan Fernandez, P24 LANL Overview of research being done in p-24 -

4. Tom Intrator, P24 LANL: Intro to dynamo, reconnection, shocks

5. Bill Daughton X-CP6 LANL: Intro to computational particle in cell methods

6. Kirk Flippo, P24 LANL: High energy density plasmas

7. Thom Weber, P24 LANL: Energy crisis, fission, fusion, non carbon fuel cycles

8. Tom Awe, Sandia National Laboratory: Magneto Inertial Fusion

9. Yongho Kim, P24 LANL: Industrial technologies 


\title{
What is a plasma?
}

\section{The other $99 \%$ of the universe}

\author{
T. Intrator \\ P-24 Plasma Physics \\ 2012 Jun 13
}

P-24 Plasma Physics Summer School

Center for Non Linear Studies conference room

Wednesdays $1 \mathrm{pm}-2 \mathrm{pm}$ 


\section{Abstract}

This introduction will define the plasma fourth state of matter, where we find plasmas on earth and beyond, and why they are useful. There are applications to many consumer items, fusion energy, scientific devices, satellite communications, semiconductor processing, spacecraft propulsion, and more. Since $99 \%$ of our observable universe is ionized gas, plasma physics determines many important features of astrophysics, space physics, and magnetosphere physics in our solar system. We describe some plasma characteristics, examples in nature, some useful applications, how to create plasmas. A brief introduction to the theoretical framework includes the connection between kinetic and fluid descriptions, quasi neutrality, Debye shielding, ambipolar electric fields, some plasma waves. Hands-on demonstrations follow. More complete explanations will follow next week. 


\section{Outline}

- What is a plasma?

- Different examples

- How to create plasmas?

- Theoretical framework

- Particles \& fluids

- Maxwell's equations

- Debye shielding, sheath
- Magneto hydrodynamics

- Waves

- Bruno Bauer next week will amplify on these and more

- Some applications

- Summary 


\section{Large Scale Plasma Structure in the Universe}
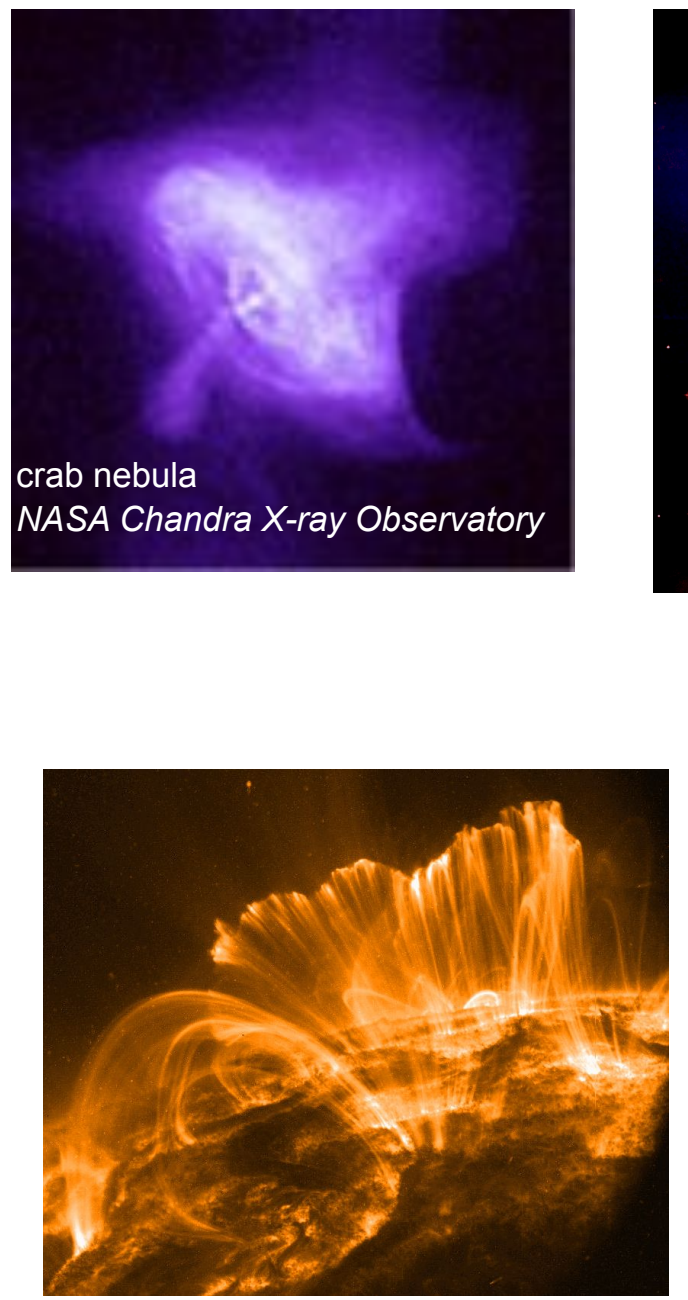

TRACE satellite VUV images

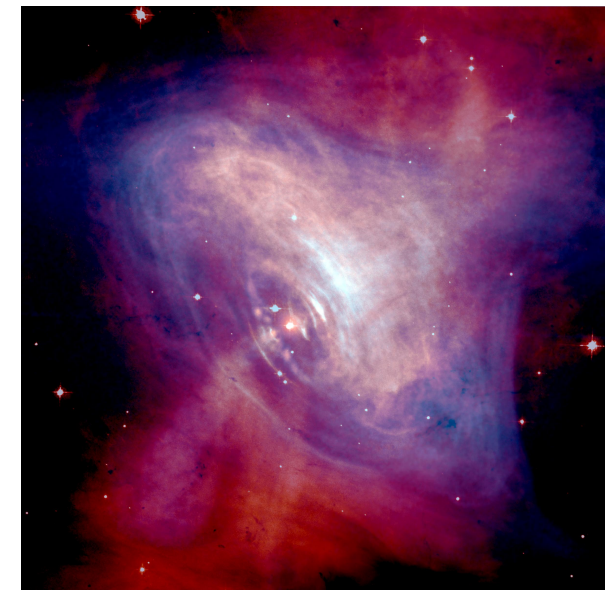

Crab Pulsar. This image combines optical data from Hubble (in red) and $X$ ray images from Chandra $X$-ray Observatory (in blue)

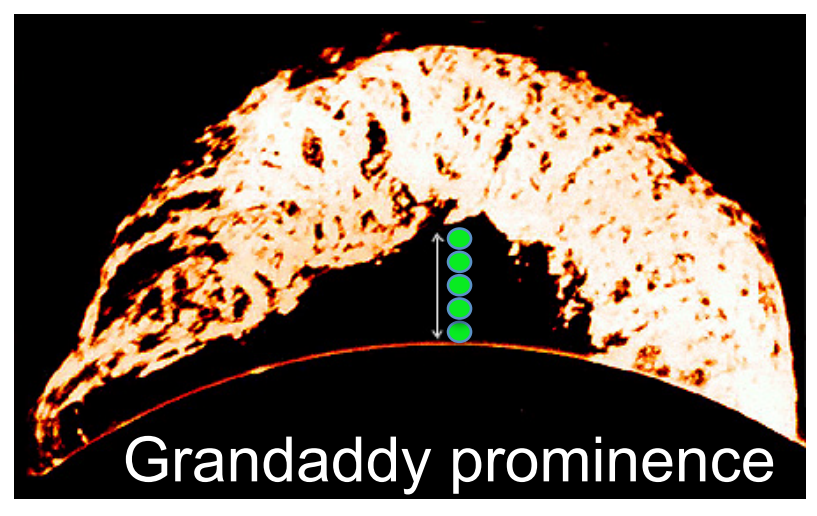

Arrow is $64,000 \mathrm{~km}, 5$ earths high, earth diameter $=$ $12756 \mathrm{~km}$

June 4, 1946 High Altitude Observatory... http://solar-center.stanford.edu/compare

Coronal arches, magnetic structures 


\section{What is a plasma?}

- Plasma state is the "fourth" state of matter

- As a solid is heated

- Bonds between adjacent molecules loosen $\quad \Rightarrow$ solid (1/40 eV)

- More heat $=>$ loosens up the lattice $\quad \Rightarrow>$ Liquid state

- More heat => neighboring bonds are broken => gas

- More heat $=>$ molecular collisions $\quad \Rightarrow$ dissociate to atoms

- More heat $=>$ collisions knock off electrons $\quad=>$ plasma $(>2 \mathrm{eV})$

- neutral particles, ions \& electrons

- $\mathrm{k}_{\mathrm{B}} \mathrm{T} \approx$ few eV, equivalent to chemical bonds

- $99 \%$ of the universe

- solid => liquid => gas => plasma 


\section{Plasma science is interdisciplinary}

- Plasma astrophysics: most of our universe

- Planetary magnetospheres

- Fusion energy

- Magnetic: tokamak, stellarator, compact toroids: MFE

- Inertial: laser, Z-pinch, pulsed: IFE

- Magneto-Inertial: MIF is in between

- High energy density plasmas, extreme states of matter

- Spacecraft thrusters

- Industrial applications: semiconductors

- Radiation generators

- Weapons 


\section{Normal atoms are charge neutral}

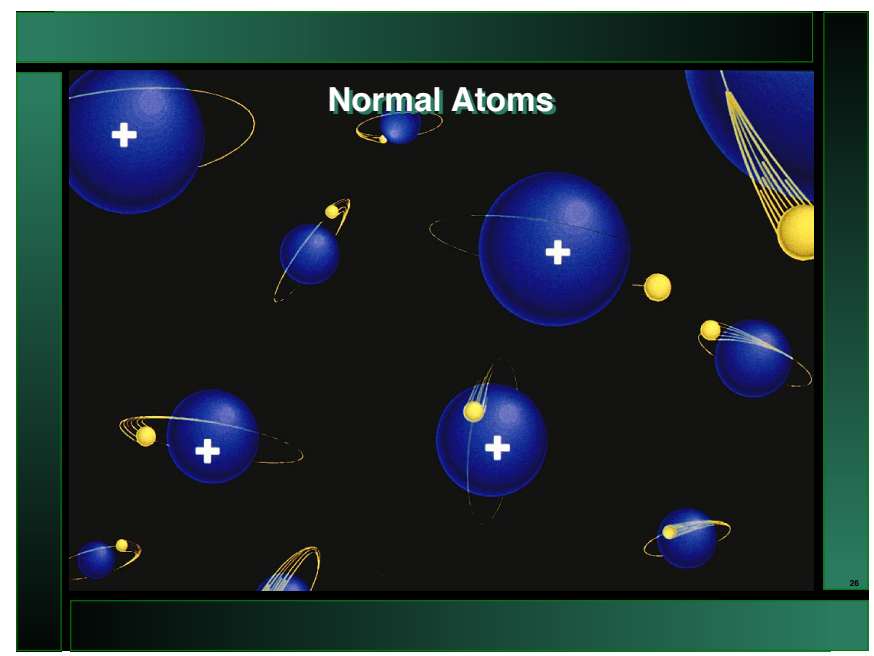

Add some thermal energy electrons acquire enough energy to escape from atomic binding forces $=>$ ions \& electrons

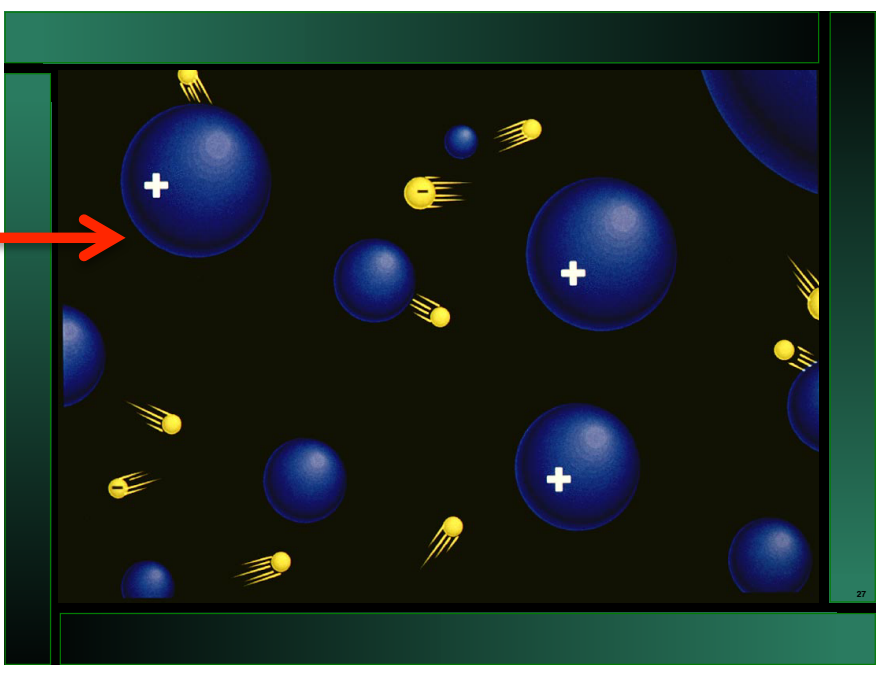




\section{plasmas differ from everyday materials}

- With most materials, nearest neighbor interactions determine properties

- BUT: In a plasma,

- charge separation generates electric fields

- Charge flow generates currents and magnetic fields

- These fields create "action at a distance" - Collective effects

- A huge range of phenomena

- Startling complexity

- Many practical applications

- Observable $99 \%$ of the universe is plasma 


\section{Plasmas - The $4^{\text {th }}$ State of Matter}

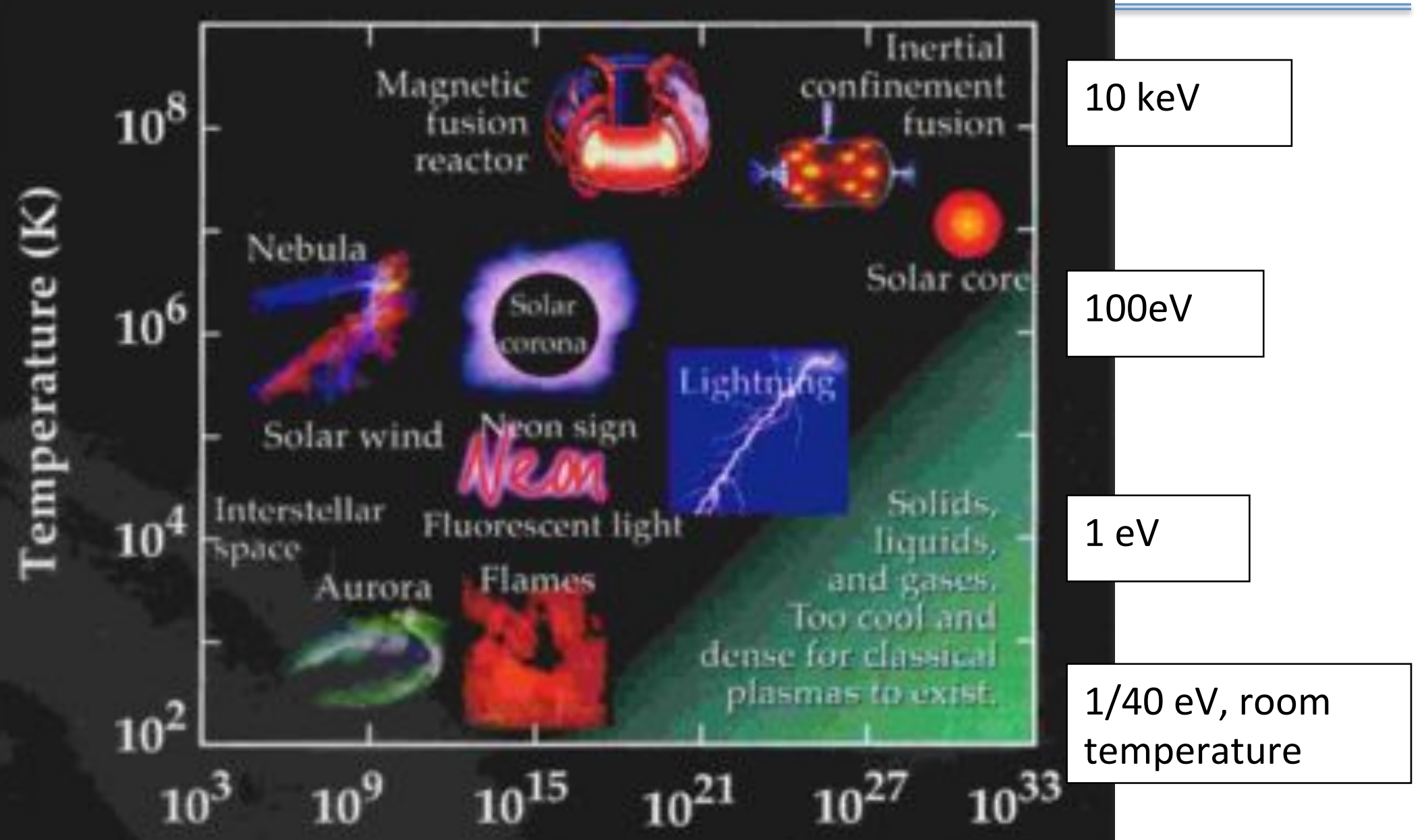

Number Density (Charged Particles $/ \mathrm{m}^{3}$ ) 


\section{Plasma - $\pi \lambda \alpha \sigma \mu \alpha$}

- The term plasma was originally coined by Langmuir and Tonks in 1929

- $\pi \lambda \alpha \sigma \mu \alpha$

- In Greek, this means moldable substance or jelly

- Today the term "plasma" is used quite generally to describe quasi neutral systems of charged particles

- Plasma physics is the study of the its behavior

- Analogous to quantum mechanics - both wave and particle properties

- Plasmas behave both as collections of particles as well as a fluid

- Fluid and electromagnetic waves, flows 


\section{Sketch the history}

- 1879 Crookes tube - "radiant matter"

- 1920's Langmuir- vacuum tubes

- 1930's Appleton- ionosphere

- 1950's thoughts about nuclear fusion

- 1960's solar wind, stellar interiors

- 1980's ... industrial applications

- Semiconductor industry

- Plasma processing of materials

- Plasma torch 


\section{Where do we find plasmas?}

- On earth in nature

- Lightning

- Aurora

- in everyday life

- Spark plug, arc welder, gas stove, fluorescent light bulb, ...

- Beyond our earth's atmosphere

- Magnetosphere pervades our solar system

- Plasma system formed by the interaction of the earth's magnetic field and the solar wind

- Stars are made of plasma, including our sun

- Most of the universe is made up of plasma

- Leave dark matter for the cognoscenti 


\section{Northern lights - aurora borealis}

electron acceleration from $1 R_{E}$ down polar field lines 


\section{How to make a plasma?}

- Heating a container with gas inside is not practical

- the container would need to be as hot as the plasma, i.e. ionized as well

- Typically in the laboratory a small amount of gas is heated and ionized. Power absorbtion can occur when you

- Pass a current through it

- Sprinkle a gas with fast electrons to collide with atoms

- Shine radio, light waves through it

- Shock it

- Container must be cooled, or insulated (e.g. with a magnetic field) 


\section{What are plasmas used for?}

- Gas lasers, visible, X-ray wavelengths

- Plasma processing of materials

- Plasma assisted chemistry

- etching \& deposition (semi-conductors, sunglasses)

- Lighting

- Next generation of particle accelerators

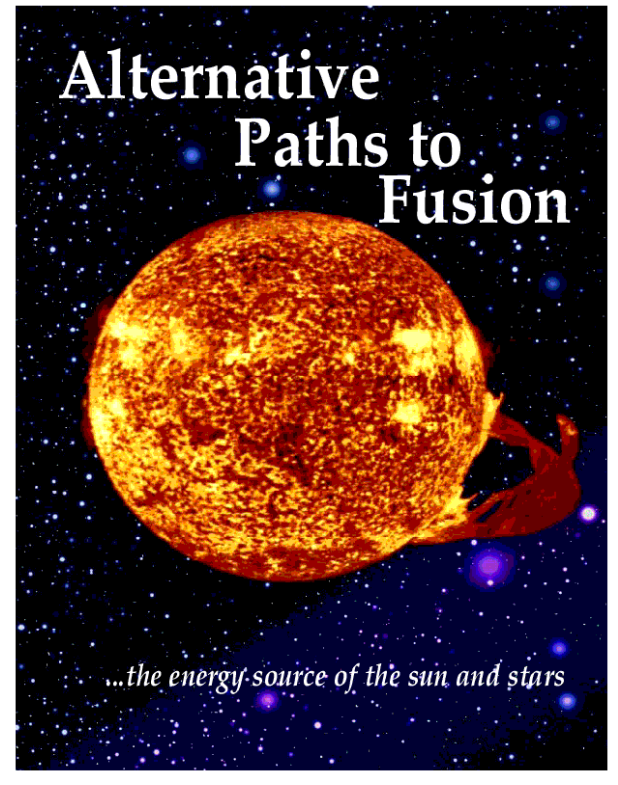

- Potential energy source from thermonuclear fusion

$-\mathrm{D}^{+}+\mathrm{T}^{+}=>\alpha(3.5 \mathrm{MeV})+\mathrm{n}(14.1 \mathrm{MeV}), \mathrm{T}_{\mathrm{i}}>10 \mathrm{keV}$

- Magnetic confinement, laser inertial confinement, Magneto Inertial Fusion

- Non carbon fuel cycle

- "greener" than fission breeder reactors 


\section{Plasma processing of materials}

- Plasma chemistry

- Surface modification

- Semi conductors

- Etching

- Oxiding

- Plasma spray, torch

- Decontamination

- Gas lasers

- Solid state plasmas

- Fusion

- Space physics

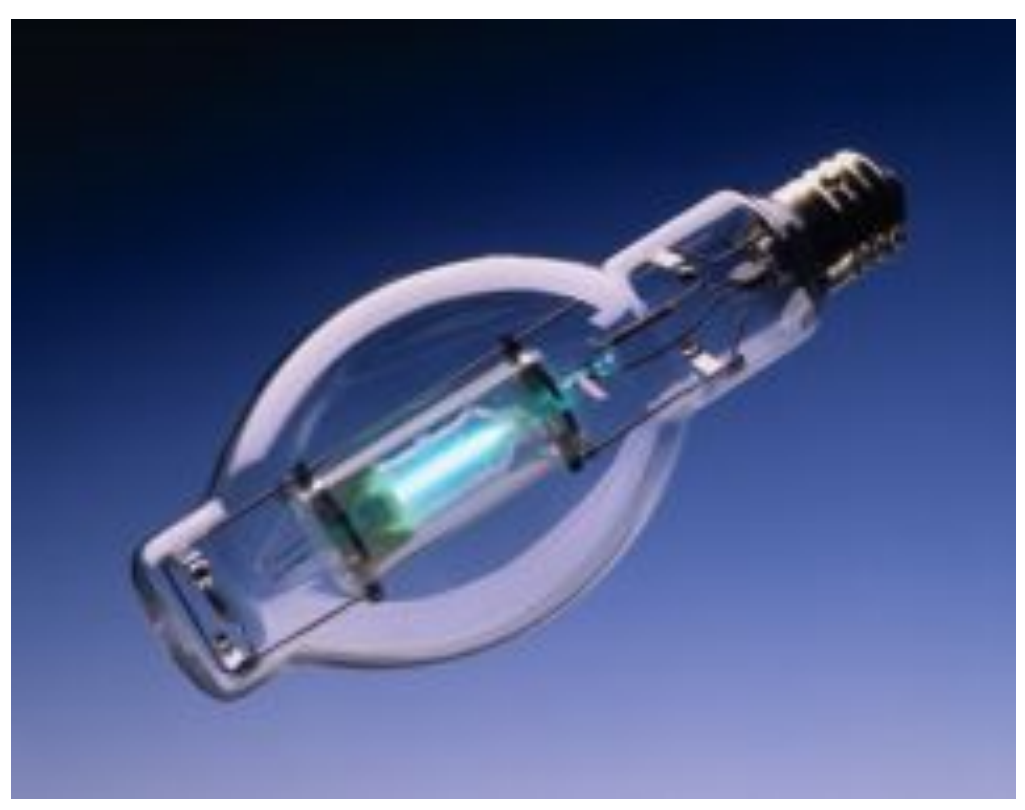




\section{Why fusion plasma science?}

- Dual nature of fusion research

- Grand Challenge frontiers of science and technology

- Plasma physics of most of the baryonic universe

- Non-fossil energy source ... could power large cities and industries

- LANL missions

- Energy security

- Threat reduction: neutrons for active interrogation, interpret satellite data

- Pushes limits of multi scale computing

- NWP: high energy density plasmas

- many other applications

- Astro, space, solar physics

- Advanced space propulsion 


\section{Theoretical framework is diverse}

- Start with gas with charged particles

- Kinetic theory of gases can be extended, include magnetic fields

- Energy transfer between waves and particles

- Many body problem for inverse square fields

- Coulomb for charged particles $\mathrm{q} / \mathrm{r}^{2}$

- Gravity for stars mMG/ $\mathrm{r}^{2}$

- Fluid dynamics with high conductivity

- Magneto Hydro Dynamics - (MHD)

- Flows convect magnetic fields, relaxation, self organization

- Similarity to a solid

- Collective wave modes are described by a dielectric tensor

- Key properties- polarization, dispersion resemble solid state physics 


\section{D view of solar wind, bow shock}

probably typical of planetary magnetospheres

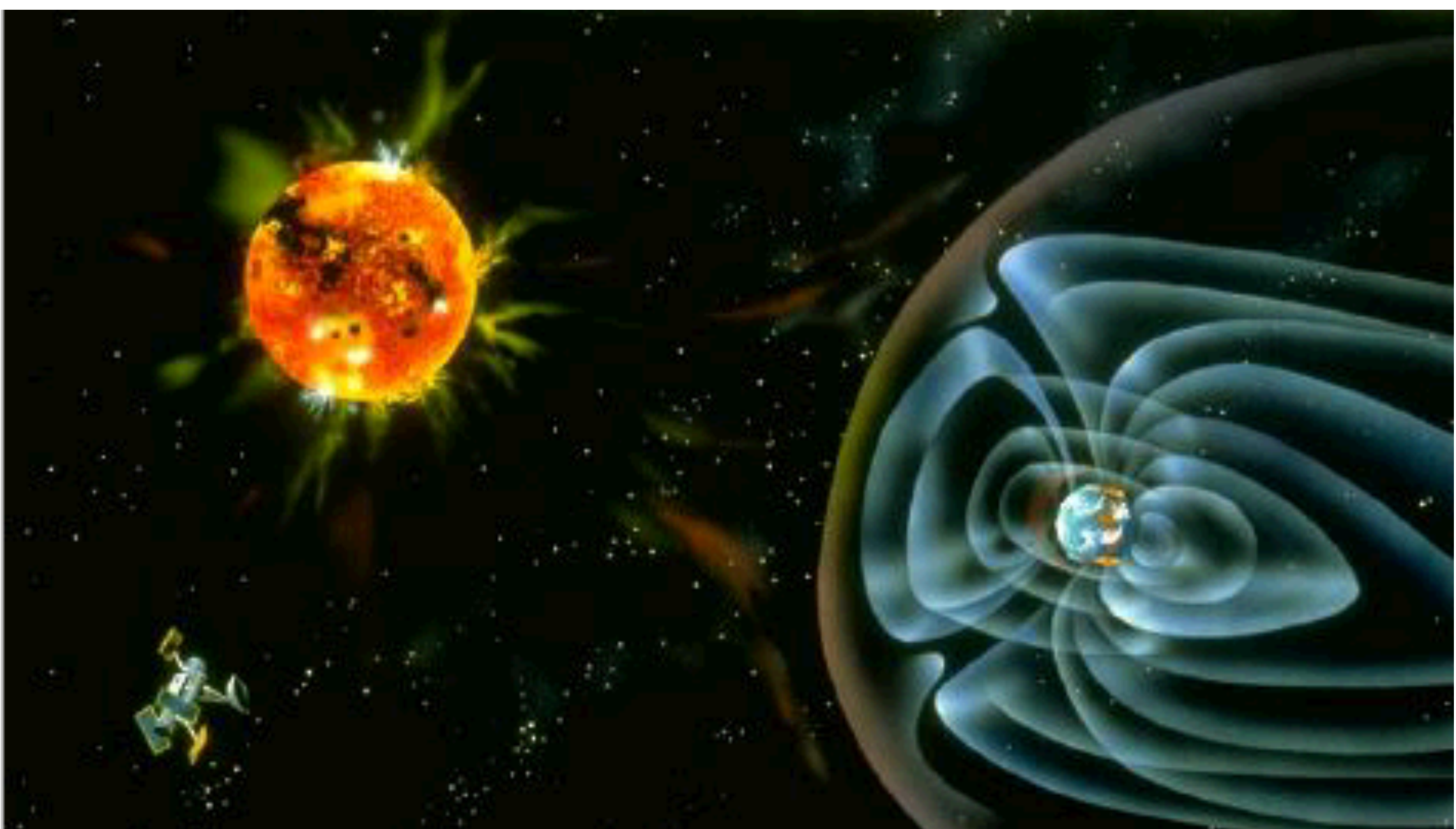

Fluid like flow in the solar wind 


\section{Fluid "feels" electromagnetic forces}

- Plasma fluid includes

- High electrical conductivity

- free positive and negative charges < => electric fields

- Charge movements $<=>$ currents

- Currents \& magnetic fields must close on themselves

- Plasma electric and magnetic fields

- This leads to a diverse "zoo" of properties

- Self consistent structure is fascinating

- Vorticity, particles <=> waves, turbulence, chaos ...

- Self organization 


\section{Maxwell' s equations (SI units)}

describe electromagnetic fields

$\begin{array}{ll}\text { Faraday' s Law } & \nabla \times E=-\frac{\partial B}{\partial t} \\ \begin{array}{l}\text { Loop currents and } \\ \text { magnetic field }\end{array} & \nabla \bullet B=0 \\ \begin{array}{l}\text { Poisson' s eqn, } \\ \text { charge density }\end{array} & \nabla \bullet \varepsilon_{0} E=e\left(n_{i}-n_{e}\right)=\rho \\ \text { Ampere' s law } & \nabla \times B=\mu_{0} J+\frac{1}{c^{2}} \frac{\partial E}{\partial t}\end{array}$

Current conservation

$\partial($ en $) / \partial t+\nabla \cdot \jmath=0$

Lorentz force equation

$F=q(E+v x B)$ 
Earth + solar wind + dipole magnetic field => convecting field lines, bowshock, magnetotail, plasmoid

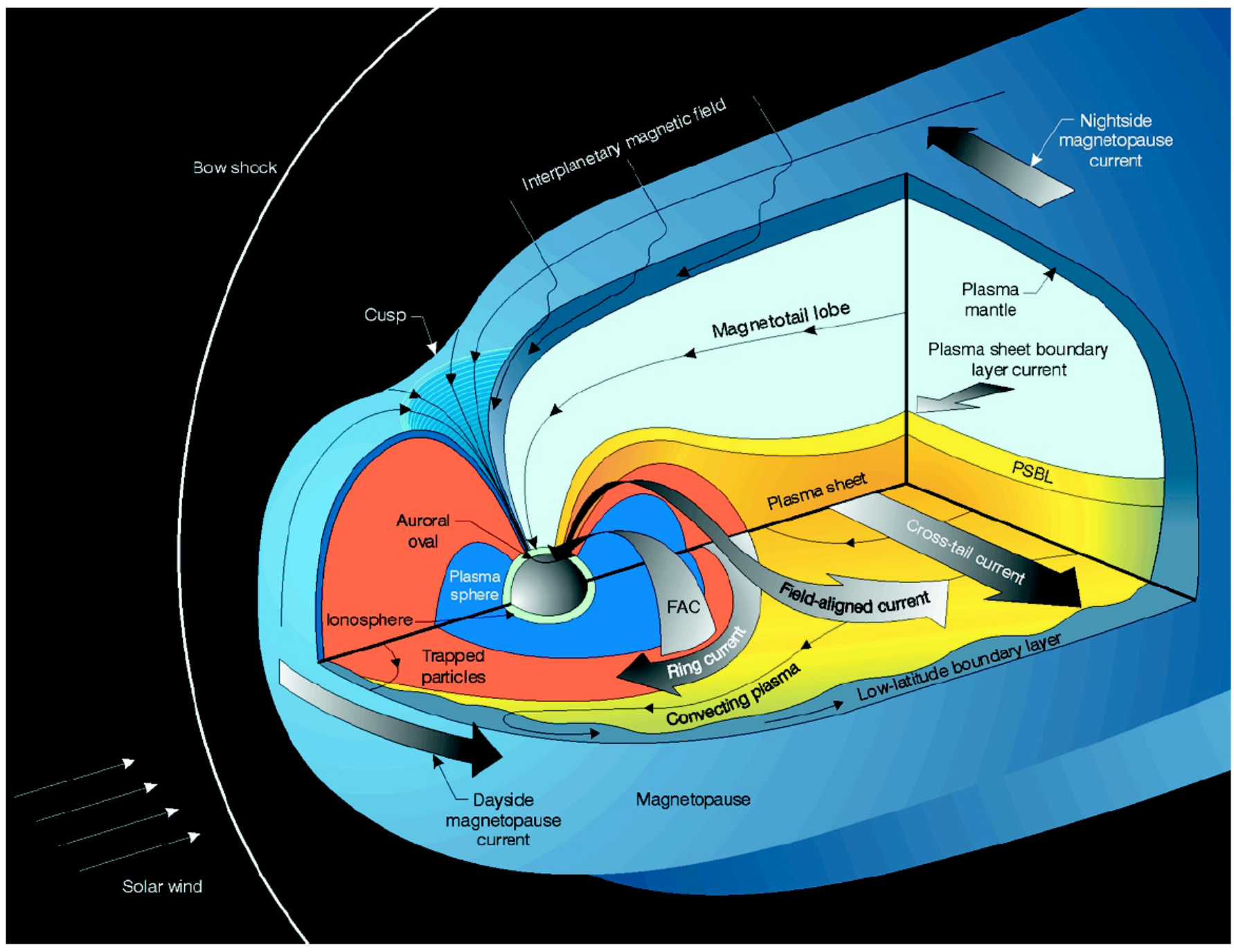




\section{Link particle and fluid pictures}

- Velocity distribution function

$-f_{v}(x, t)$

- Probability of a particle with a chosen velocity

- Thermal jitter $=>$ Maxwellian distribution

$-f(v)=$

- $\left(2 \pi v_{\mathrm{th}}{ }^{2}\right)^{-1 / 2} \exp \left(-\left(v-v_{\mathrm{D}}\right)^{2} / 2 \mathrm{v}_{\mathrm{th}}{ }^{2}\right)$

- $\mathrm{T}=\mathrm{mv}_{\mathrm{th}}{ }^{2}$

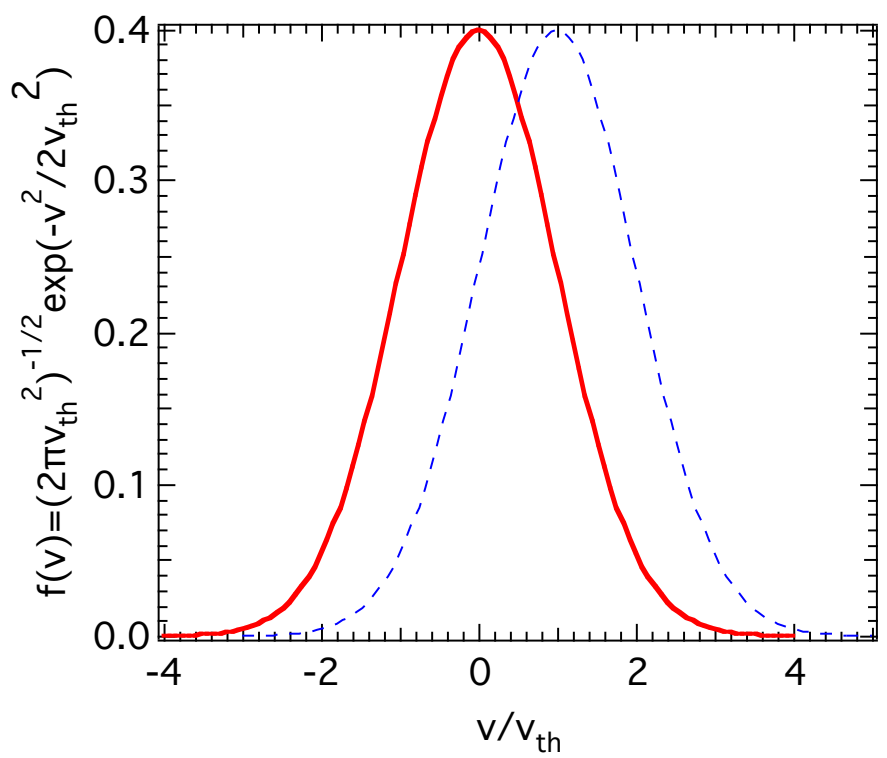

Averaged fluid quantities

- Density $n / n_{0}=\int f(v) d v$

- Thermal velocity $v_{\text {th }}{ }^{2}=\left\langle v^{2}\right\rangle=\int v^{2} f(v) d v$

- Drift velocity $v_{D}=\langle v\rangle=\int v f(v) d v$ 


\section{Charge neutrality \& Debye length}

- Consider a plasma, that initially has

- Thermo dynamic equilibrium: Maxwell-Boltzmann distribution

- uniform charge density $\mathrm{n}_{\mathrm{i} 0} \approx \mathrm{n}_{\mathrm{e} 0}$

- zero electric field

- How does the charge density behave when we attempt to change it?

- Suppose electron density drops from $n_{\mathrm{e} 0}=>(1-\delta) \mathrm{n}_{\mathrm{e} 0}, \mathrm{n}_{\mathrm{i}}(\mathrm{x}) \approx$ constant

- If there were no change in electron density the equation for potential would be

$-\mathrm{E}=-\nabla \phi$

$-\nabla \bullet \varepsilon E=e\left(n_{i}-n_{e}\right) \quad$ divergence of $D=\varepsilon E=$ free charge density

$-\quad-\varepsilon \nabla^{2} \phi \quad=e \delta n$

$-\mathrm{n}_{\mathrm{e}}(\phi, v)=\mathrm{n}_{\mathrm{e} 0} \exp \left[-\left(\mathrm{e} \phi / T+\mathrm{v}^{2} / 2 \mathrm{v}_{\mathrm{th}}{ }^{2}\right)\right]$ 


\section{Charge neutrality \& Debye length}

- Maxwellian electron distribution $\mathrm{f}($ energy) in 1 dimension

$-\quad-\varepsilon d^{2} \phi / d x^{2}=e\left\{n_{i 0}-n_{e 0} \exp \left[-\left(e \phi / T+v^{2} / 2 v_{t h}{ }^{2}\right)\right]\right\} \&$ integrate over velocity

$-\mathrm{n}(\mathrm{x}) / \mathrm{n}_{\mathrm{e} 0}=\exp \left[-\left(\mathrm{e} \phi(\mathrm{x}) / \mathrm{T}_{\mathrm{e}}\right)\right]$

- expand the ODE in terms of small $\phi / T$,

- $-\varepsilon \mathrm{d}^{2} \phi / d x^{2} \quad \approx \mathrm{e}\left[\mathrm{n}_{\mathrm{i} 0}-\mathrm{n}_{\mathrm{e} 0}(1-\phi / \mathrm{T})\right]$

- $\mathrm{d}^{2} \phi(\mathrm{x}) / \mathrm{dx^{2 }} \approx-\mathrm{e} \delta \mathrm{n}_{\mathrm{e} 0}(\mathrm{e} \phi(\mathrm{x}) / \mathrm{T}) / \varepsilon$

- Exponentially decaying solutions, shielded Yukawa potential

$-\phi(x) \approx \exp \left[-x / \lambda_{D}\right]$

- $\quad \mathrm{e}(\mathrm{x}) / T \approx \exp \left[-\mathrm{x} / \lambda_{\mathrm{D}}\right]$ normalize this equation to be dimensionless

- $\lambda_{\text {De }}=\left[\varepsilon_{0} \mathrm{~T} /\left(\mathrm{e}^{2} \mathrm{n}_{\mathrm{e}}\right)\right]^{1 / 2}$ Debye length $\lambda_{\text {De }}(\mathrm{cm})=740\left[\mathrm{~T}_{\mathrm{e}}(\mathrm{eV}) / \mathrm{n}\left(\mathrm{cm}^{-3}\right)\right]^{1 / 2}$

- Debye length $\lambda_{D}$ is the characteristic exponential decay length

- Potential and electric fields from a point charge are shielded out by the sea of other charged particles on $\lambda_{\text {De }}$ scales

- definition of a plasma is that many particles exist in a Debye sphere

- $\mathrm{n} \lambda_{\mathrm{D}}^{3}>>1$

- Validate statistical assumption of small departures from equilibrium 


\section{Quasi neutrality, ambipolar electric fields, sheath, boundary layer problem}

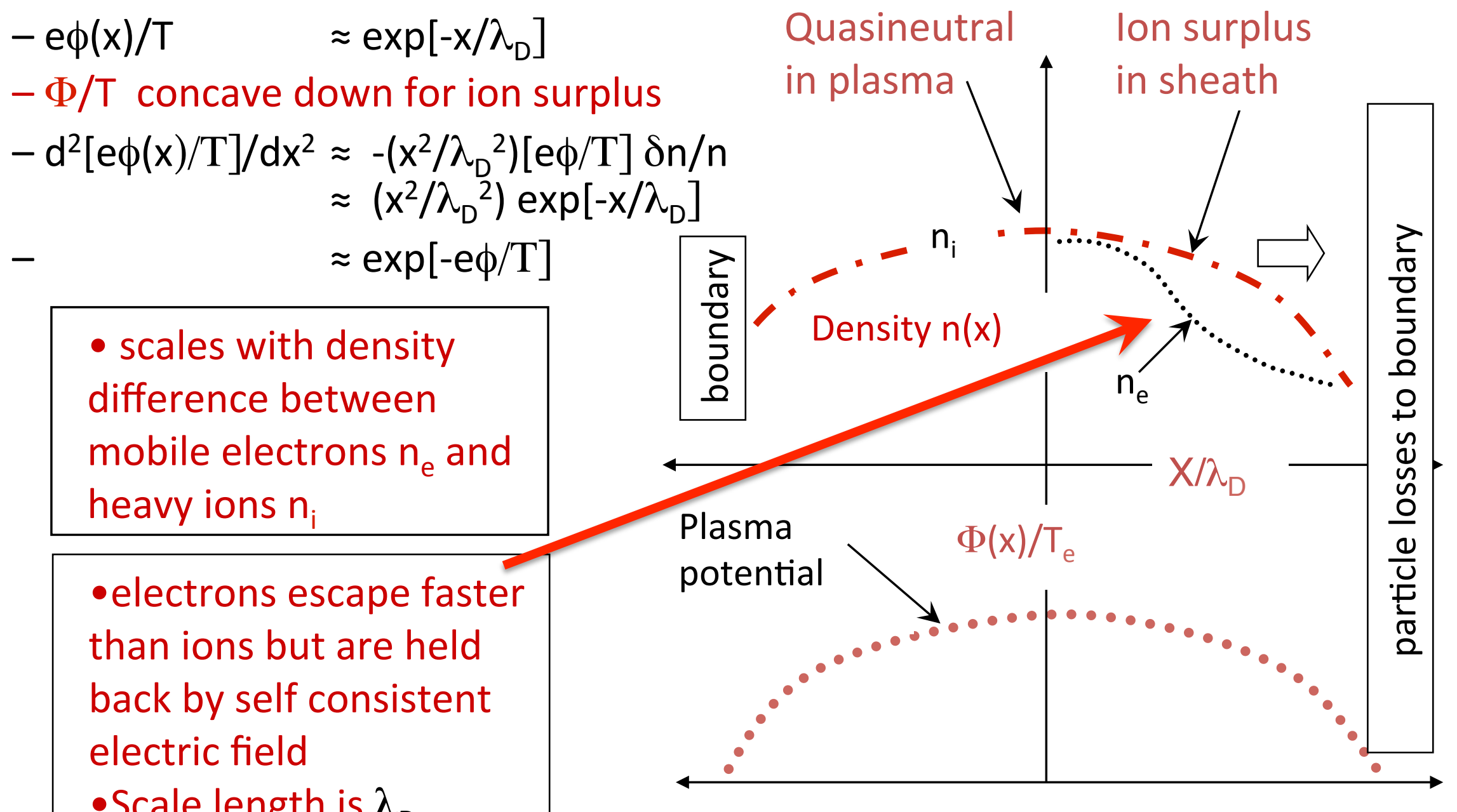


Galactic jets - fluid flow and radiation powered by annihilation of magnetic fields (reconnection)?

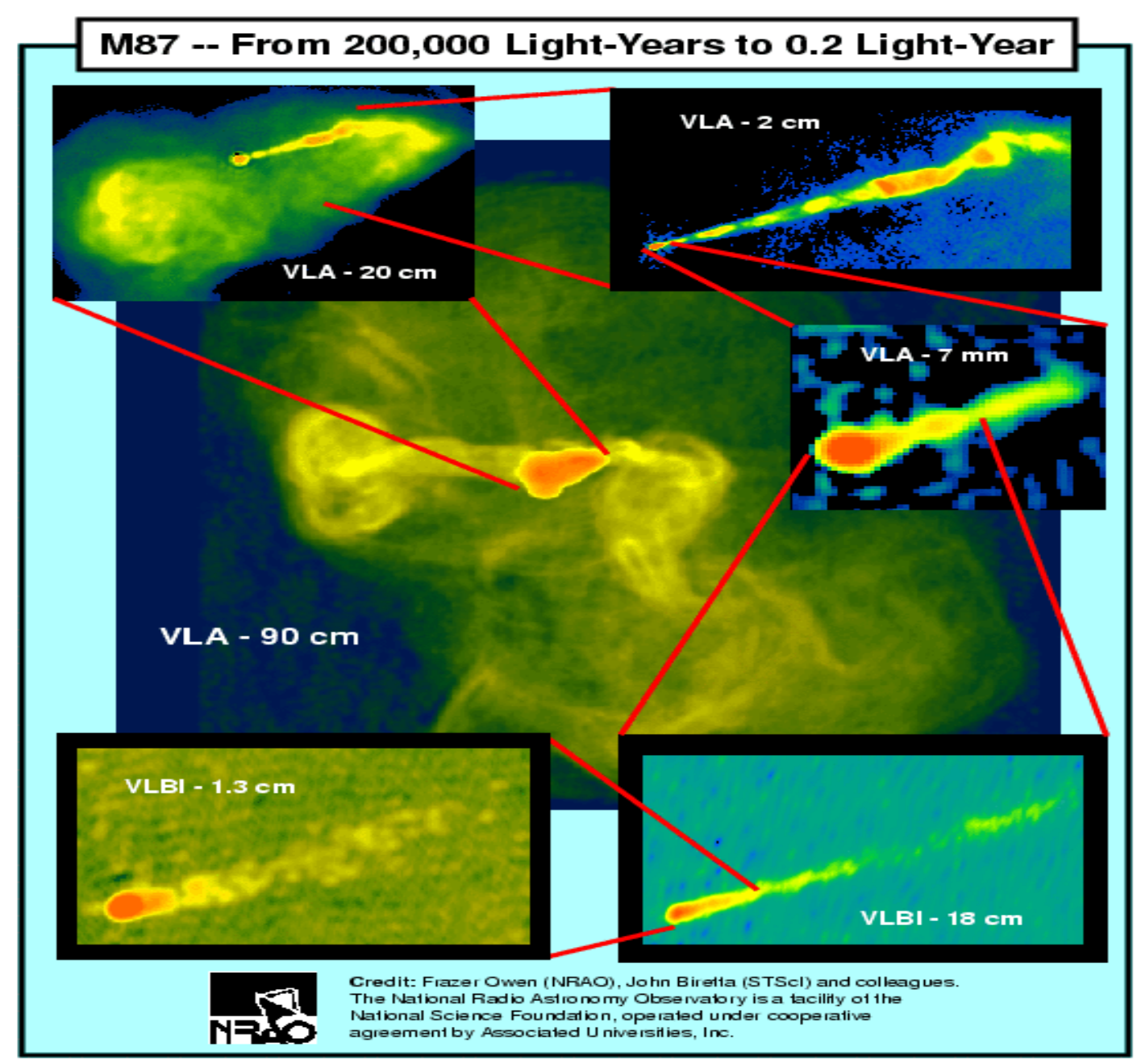




\section{Magneto Hydro Dynamic approximation}

- If a magnetized plasma has disturbances that are slow enough and large enough spatial scale

- Mobile electrons can prevent the buildup of any electric field that moves with the plasma

- Fields are "stuck" in plasma reference frame - frozen flux theorem

- Higher frequency modes of oscillation and wave propagation do not play a role

- Behavior simplifies substantially

- Magneto Hydro Dynamic (MHD) approximation 


\section{MHD force balance}

- MHD model presumes a single fluid with mass density

$-\rho=\mathrm{n}_{\mathrm{i}} \mathrm{m}_{\mathrm{i}}+\mathrm{n}_{\mathrm{e}} \mathrm{m}_{\mathrm{e}} \approx \mathrm{n}\left(\mathrm{m}_{\mathrm{i}}+\mathrm{m}_{\mathrm{e}}\right) \approx \mathrm{n} \mathrm{m}_{\mathrm{i}}$

- Charge density

- $\sigma=\left(n_{i}-n_{e}\right) e$

- Mass velocity

- $\mathbf{U}=\left(\mathrm{n}_{\mathrm{i}} \mathrm{m}_{\mathrm{i}} \mathbf{u}_{\mathrm{i}}+\mathrm{n}_{\mathrm{e}} \mathrm{m}_{\mathrm{e}} \mathbf{u}_{\mathrm{e}}\right) / \rho \approx \mathbf{u}_{\mathrm{i}}+\left(\mathrm{m}_{\mathrm{e}} / \mathrm{m}_{\mathrm{i}}\right) \mathbf{u}_{\mathrm{e}}$

- Current density

- $\mathrm{J}=\mathrm{e}\left(\mathrm{n}_{\mathrm{i}} \mathrm{u}_{\mathrm{i}}-\mathrm{n}_{\mathrm{e}} \mathrm{u}_{\mathrm{e}}\right) \approx \mathrm{ne}\left(\mathrm{u}_{\mathrm{i}}-\mathrm{u}_{\mathrm{e}}\right)$

- Single fluid equation of motion

- $\rho \mathrm{du} / \mathrm{dt}=\{\partial \mathrm{u} / \partial \mathrm{t}+\mathrm{u} \bullet \nabla \mathrm{u}\}=\sigma \mathrm{E}+\mathrm{jxB}-\nabla \mathrm{p}$

- Resistive MHD includes finite resistivity

- $E+u \times B=\eta J+\{j \times B-\nabla p\} / e n$ 


\section{Current ropes modeled in the laboratory}

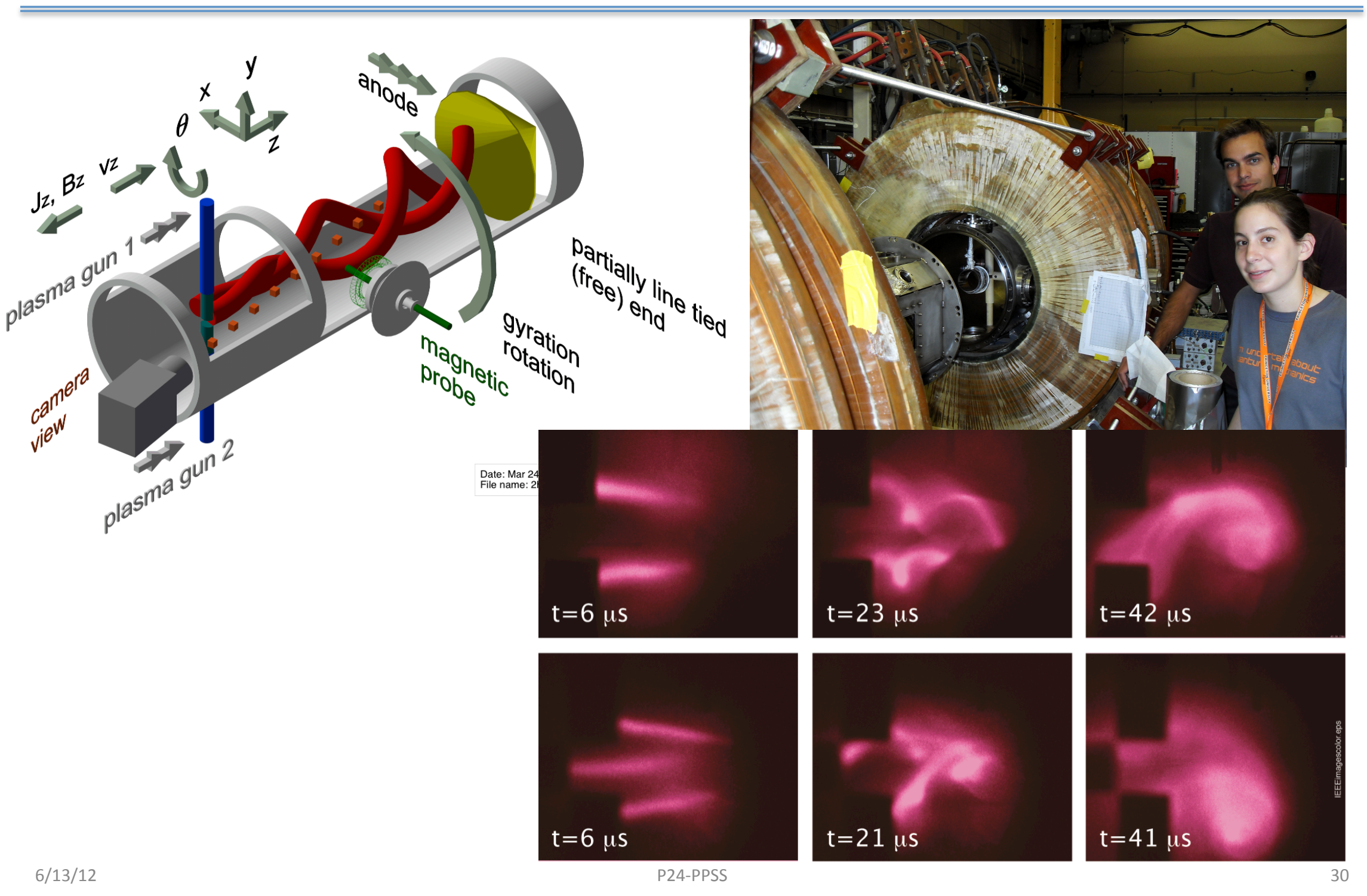




\section{Alfven waves \& MHD}

- A notable property of MHD plasmas was discovered by Hannes Alfven in 1942

- Michael Faraday had shown in 1800's that stresses in the magnetic field are equivalent to a pressure transverse to the $B$ field and $a$ tension along the field lines.

- Alfven showed that certain waves can propagate along the field

- Analogous to waves on a plucked string "Alfven waves"

- Sound and Alfven speeds 


\section{3 flux ropes in LAPD}

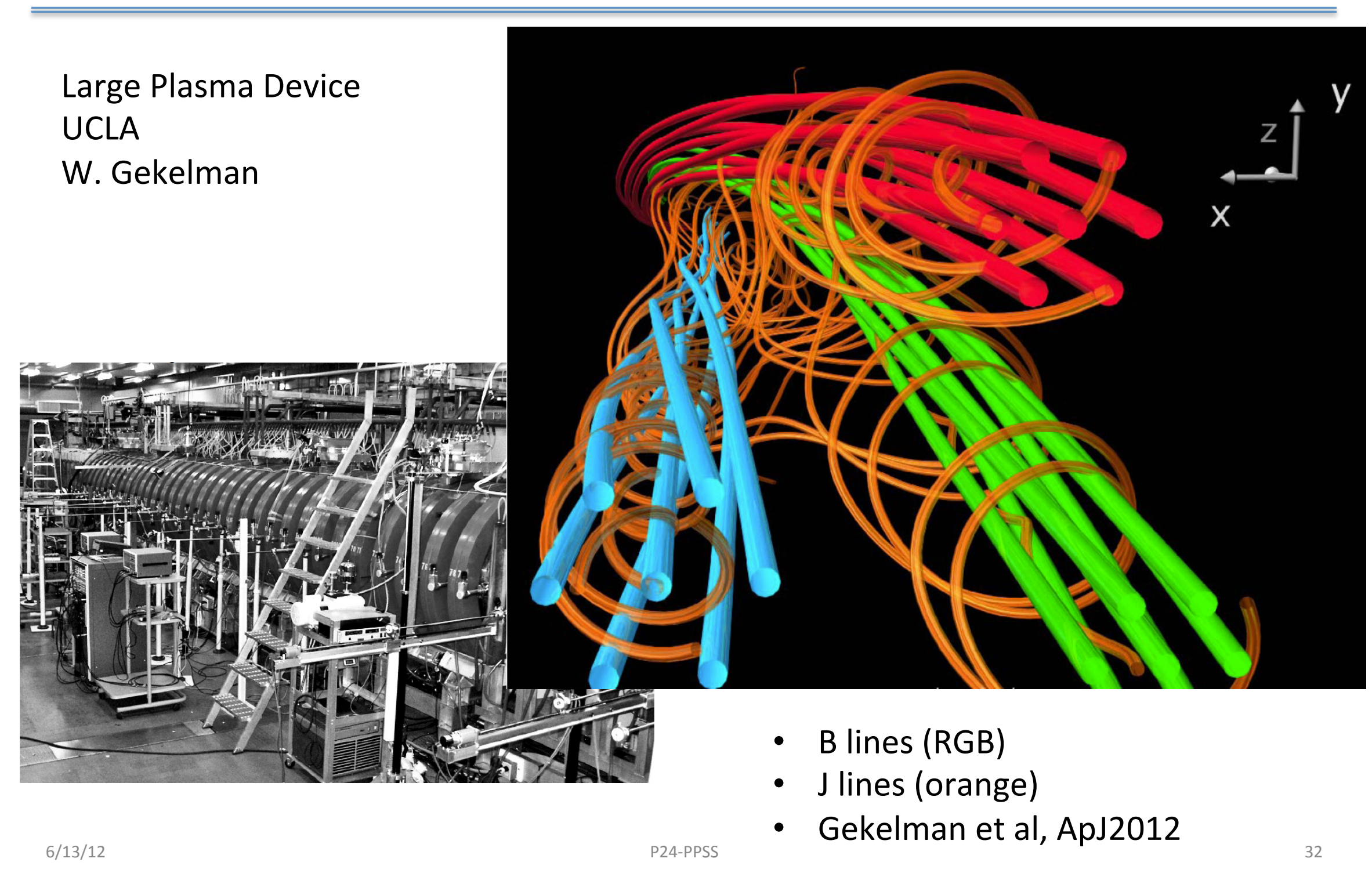




\section{Complex systems}

- Plasma systems can exhibit self organized behavior of high complexity

- Self organization occurs in many arenas

- Space \& astrophysics, biosystems, self assembly of micro and nano components, protein folding

- Selective decay processes, thermodynamics

- Dissipation of some invariant on small scales (eg energy in eddies, turbulence)

- Persistence of other quantities on larger spatial scales (e.g. helicity)

- Chaos ... a cottage industry 


\section{Solar arcades: fluid \& magnetic structure}

TRACE

spacecraft image, NASA

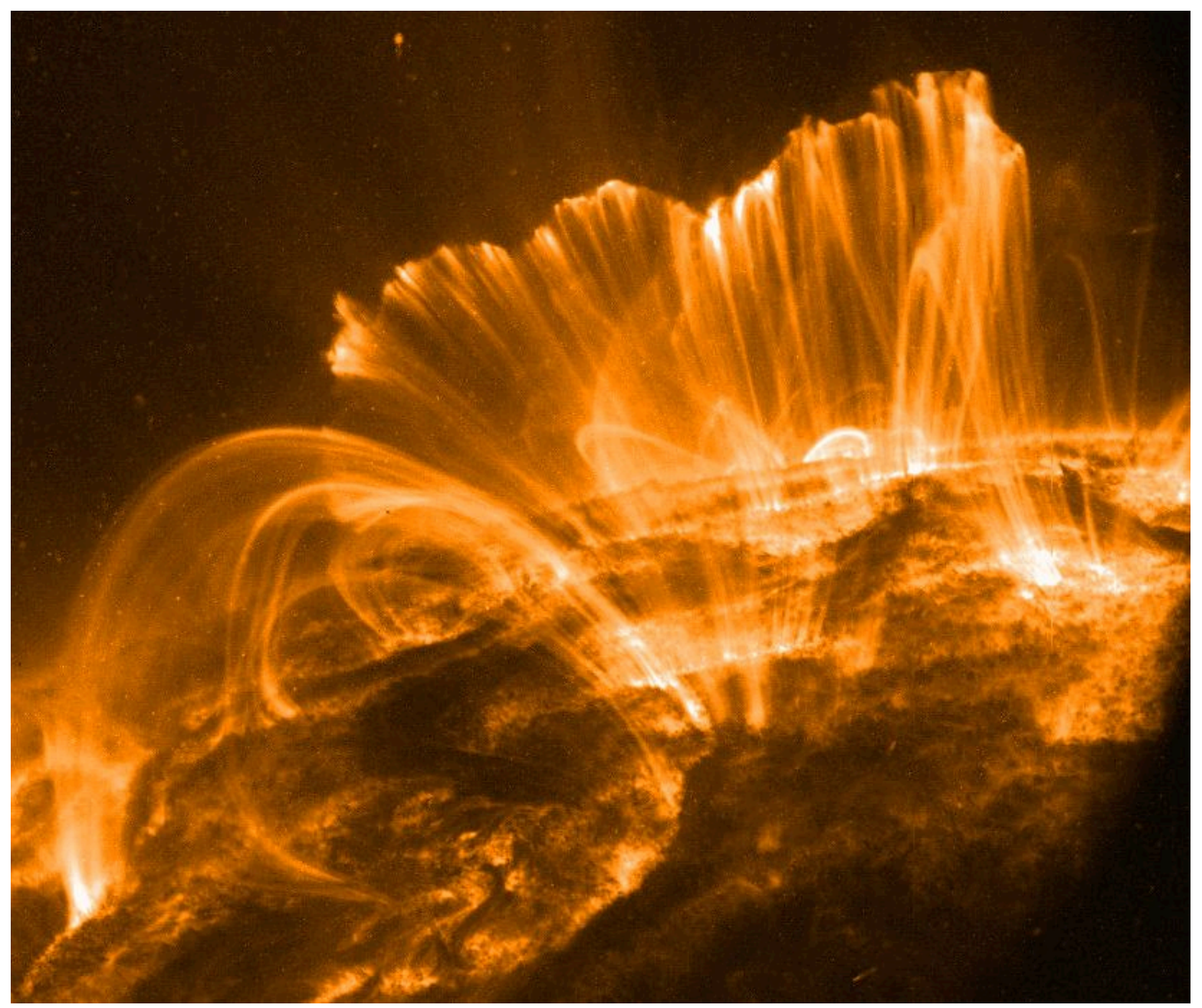




\section{Plasma etching of silicon}
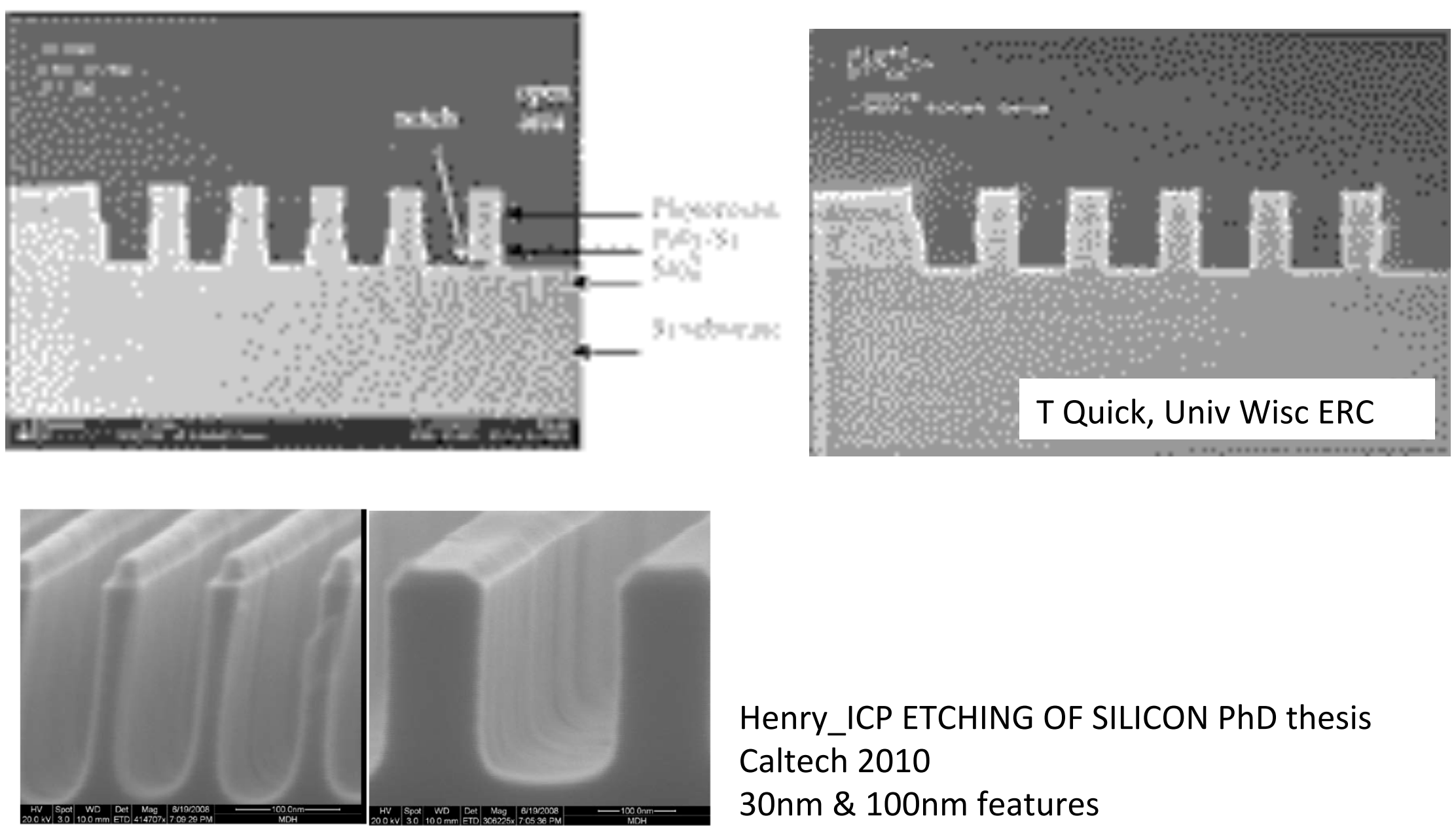

Henry_ICP ETCHING OF SILICON PhD thesis Caltech 2010

Fig II.2 Cross sectional SEM of a pseudo Bosch etch of $30 \mathrm{~nm}$ (left) and $100 \mathrm{~nm}$ (right) $30 \mathrm{~nm} \& 100 \mathrm{~nm}$ features

silicon ridges masked using approximately $55 \mathrm{~nm}$ of PMMA. This etch was terminated before the onset of mask erosion at $256 \mathrm{~nm}$. 


\section{Summary}

- Plasmas are pervasive - $99 \%$ of observable universe

- Many examples in everyday life, applications, technology, nature

- Fluids with electrical and magnetic forces

- Experimentally and computationally accessible

- Basic physics is still unfolding - an adventure 
Extra slides 


\section{Plasma waves vs free space waves}

- charged particles are tightly coupled to electric and magnetic fields in the plasma

- Wave properties depend on the the dielectric and magnetized medium

- Charged particles can move freely along a B field

- Movement across the B field is inhibited

- Anisotropic wave propagation and properties

\section{Landau Damping}

Wave propagation

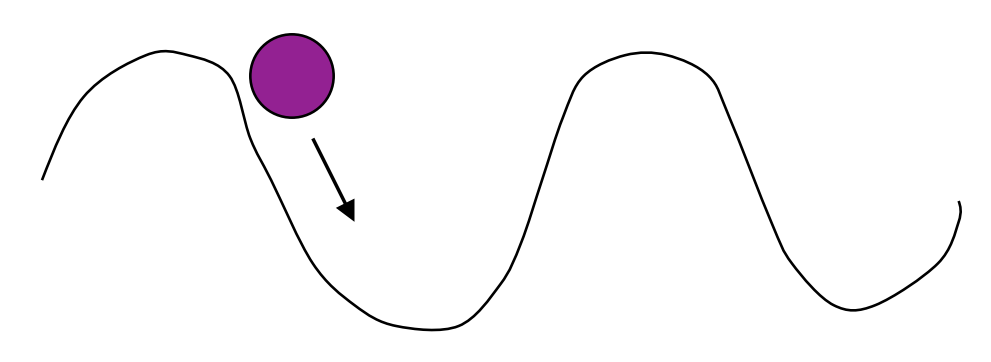

-Particles "surf" on wave if $v_{\phi}>v_{\text {particle }}$

-Wave gains energy from particle if $v_{\phi}<v_{\text {particle }}$ 


\section{Electron plasma oscillations}

- Consider one dimensional motion

- Sheet of electrons centered at $x$

- Now move e- distribution to right, $x^{\prime}=x+\xi$

- Excess positive charge ne $\xi$ per unit area

- Field points to right $\mathrm{E}=\mathrm{ne}_{0} \xi$

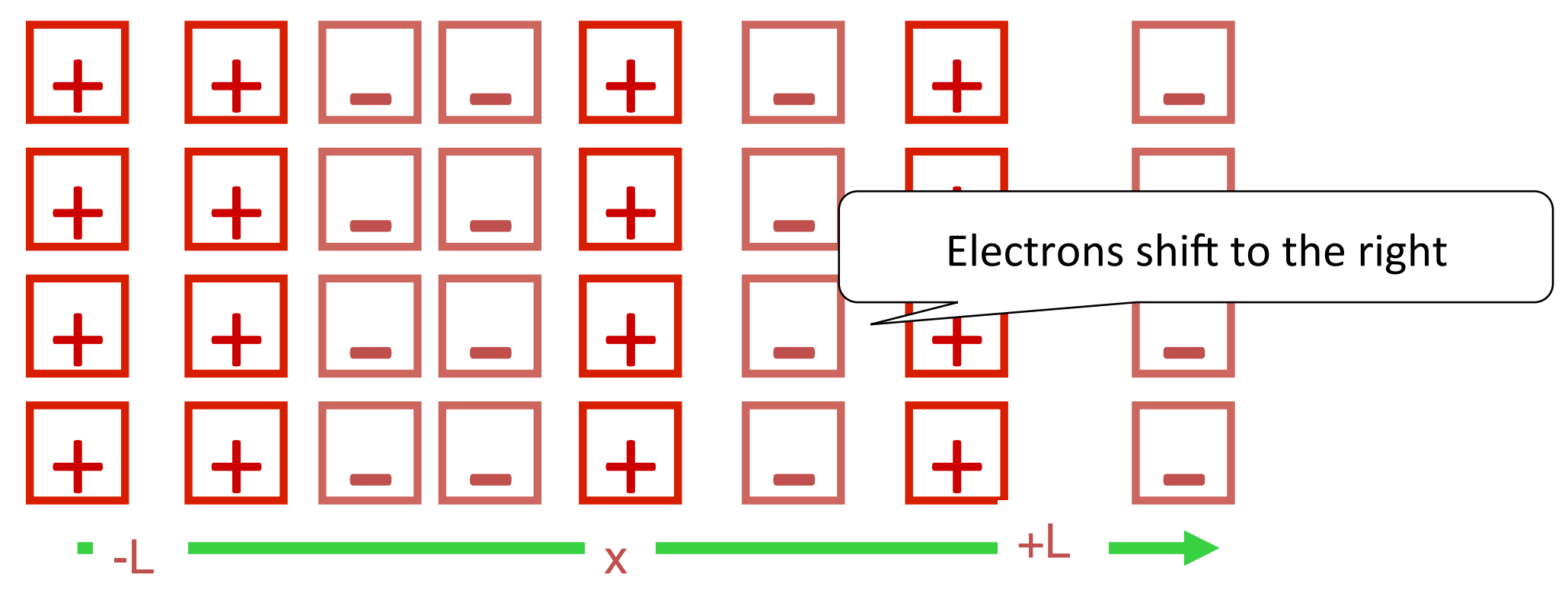

- E field points to right, because of charge separation 


\section{Electron plasma oscillations}

- Equation of motion is $\mathrm{f}=\mathrm{ma}=\mathrm{m} \mathrm{d}^{2} \mathrm{x} / \mathrm{dt}^{2}$

- $-\mathrm{eE}=\mathrm{m} \mathrm{d}^{2} \xi / \mathrm{dt}^{2}$

- Recall $E=n e \varepsilon_{0} \xi$ so that

$-m d^{2} \xi / d t^{2}=-e\left(n e \varepsilon_{0} \xi\right)$

$-d^{2} \xi / d t^{2}+e^{2} n \varepsilon_{0} \xi / m=0$

- The ODE for displacement $\xi$ is then

$-\left(d^{2} / d t^{2}+\omega_{p e}{ }^{2}\right) \xi=0$

- Where $\xi(t)=\xi_{0}\left[\exp \left(+i \omega_{p e} t\right)+\exp \left(-i \omega_{p e} t\right)\right]$

$-\omega_{p e}{ }^{2}=n \mathrm{e}^{2} \varepsilon_{0} / m$ is the electron plasma frequency 


\section{Stability}

\section{Unstable plasma systems without collisions}

- Stability is one of the most important and recurring problems in plasma physics

- Ideal plasma has zero collisions

- Certain situations may be theoretically conceivable

- But they wont survive if they are unstable

- Fusion devices, eg tokamaks, RFP,

- Buoyancy instability for flux tubes trapped in suns photosphere

- Two stream instabilities 


\section{Stability}

\section{Unstable plasma systems due to collisions}

- Finite resistivity (collisions) enables the resistive decay of currents in the system

- This is normally very slow in astrophysical settings

- However Dungey realized (1953) that resistive diffusion can lead to strong concentrations of current that then speed up the diffusion process

- Resistive instabilities

- Tearing modes, reconnection, solar flares 


\section{Complex systems}

- Plasma systems can exhibit self organized behvior of high complexity

- Self organization occurs in many arenas

- Space \& astrophysics, biosystems, self assembly of micro and nano components, protein folding

- Selective decay processes, thermodynamics

- Dissipation of some invariant on small scales (eg energy in eddies, turbulence)

- Persistence of other quantities on larger spatial scales (e.g. helicity)

- Chaos ... a cottage industry 


\section{Confinement is not always perfect (or even good)}

- Collisions between particles enable diffusion across magnetic field

- Pitch angle scattering

- Kills trapping in

- fusion devices

- Planetary magnetospheres

- Coronal flux tubes

- Collisions tend to dissipate highly ordered motions in wave propagation 


\section{START}

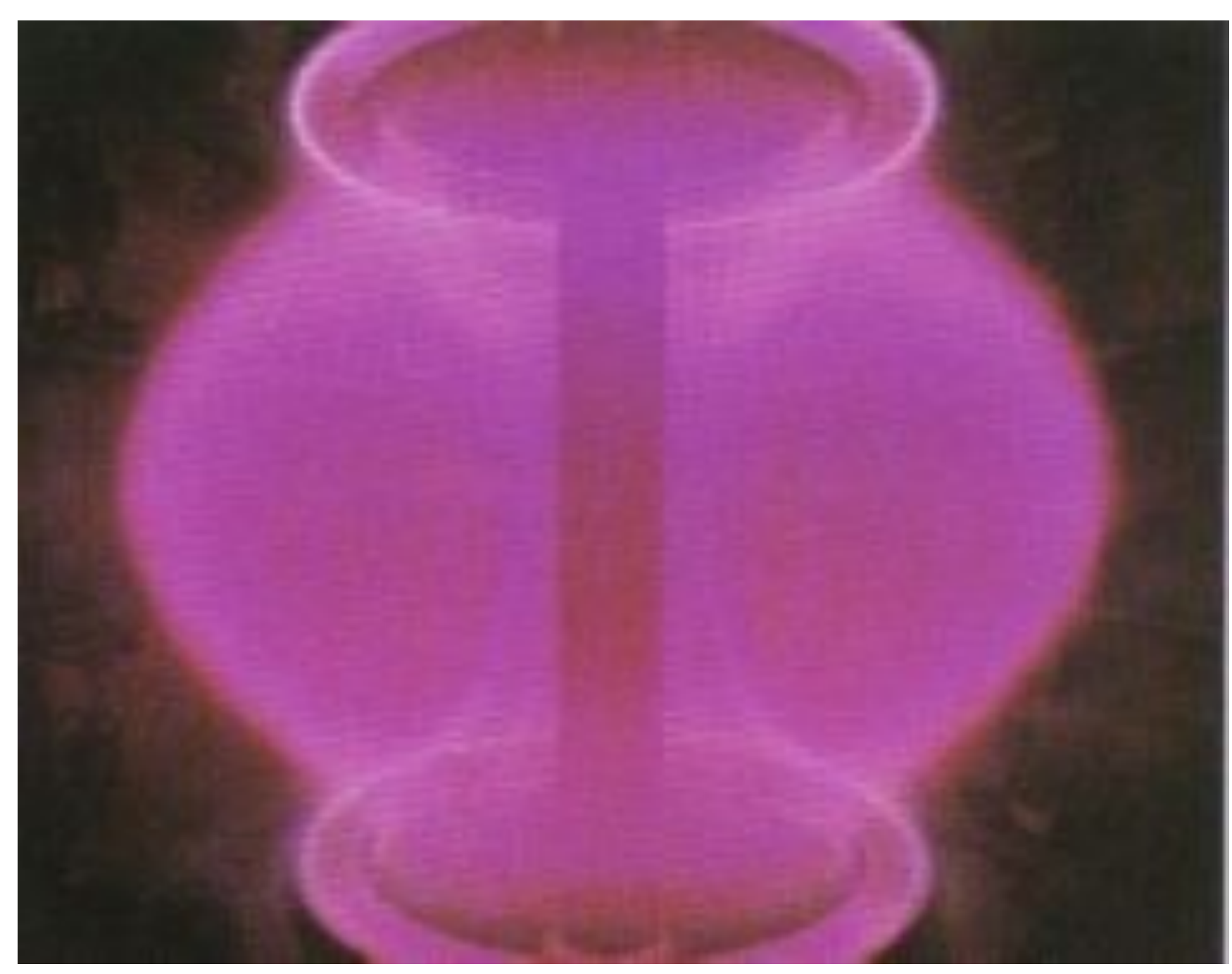




\section{Confinement means that particles leisurely linger $=>$ interact collectively}

magnetic confinement here is one example

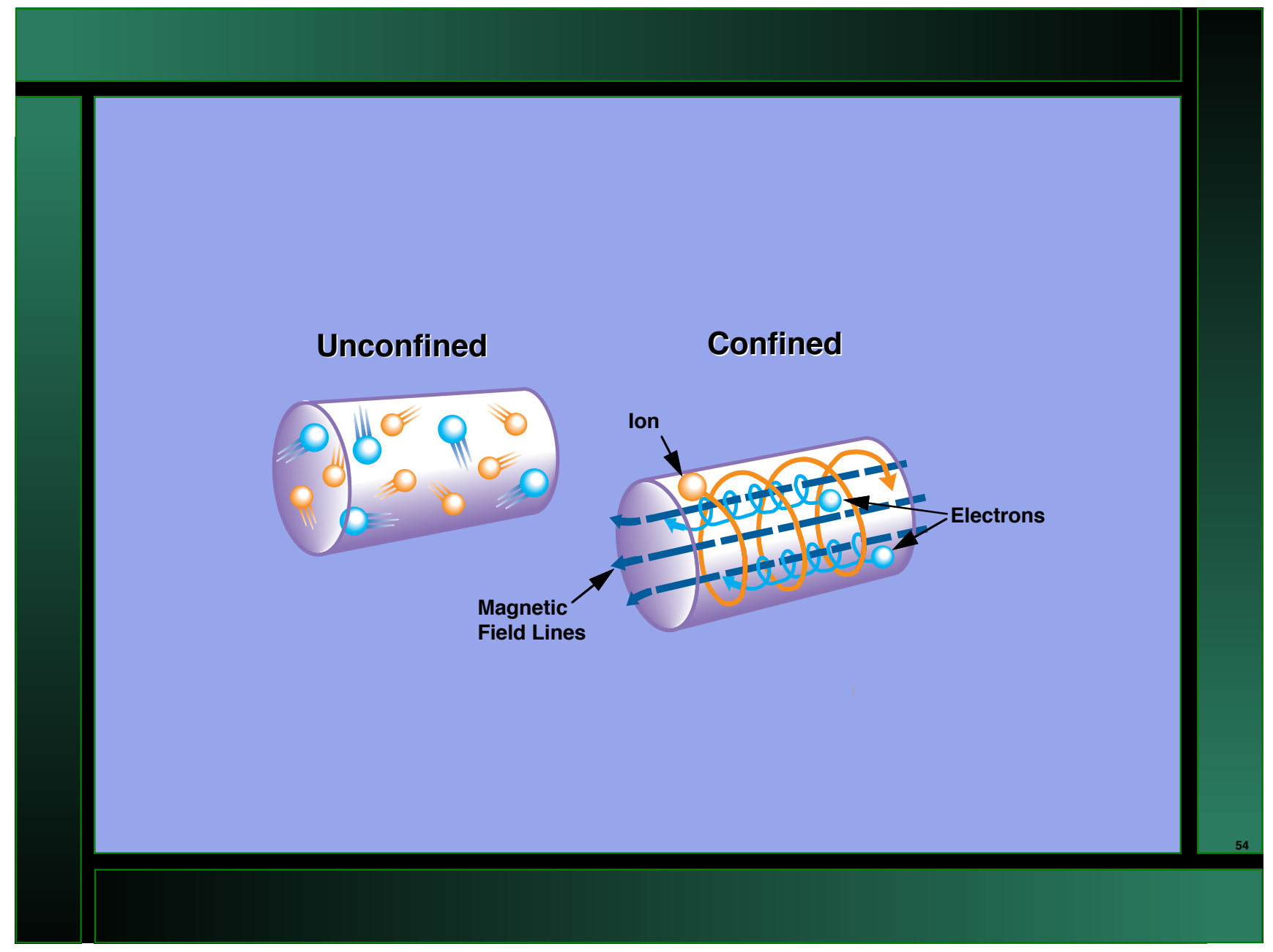




\section{Handy formulary}

- Debye length

- $\lambda_{D}(\mathrm{~cm})=740\left[\mathrm{~T}_{\mathrm{e}}(\mathrm{eV}) / \mathrm{n}\left(\mathrm{cm}^{-3}\right)\right]^{1 / 2}$

- plasma frequency

$-\mathrm{f}_{\mathrm{pe}}(\mathrm{Hz}) \quad=9000 \mathrm{n}_{\mathrm{e}}{ }^{1 / 2}\left(\mathrm{~cm}^{-3}\right) \quad$ electrons

$-\mathrm{f}_{\mathrm{pi}}(\mathrm{Hz}) \quad=210 \mathrm{n}_{\mathrm{e}}^{1 / 2}\left(\mathrm{~cm}^{-3}\right) \quad$ ions

- gyro radius

$-r_{\text {gi }}(\mathrm{cm})=100 \mathrm{~T}^{1 / 2}(\mathrm{eV}) / \mathrm{B}$ (Gauss) ions

$-r_{\text {ge }}(\mathrm{cm})=2.4 \mathrm{~T}^{1 / 2}(\mathrm{eV}) / \mathrm{B}$ (Gauss) electrons

- Thermal speed

$-v_{e, t h}(\mathrm{~cm} / \mathrm{sec})=4.2 \times 10^{7} \mathrm{~T}_{\mathrm{e}}^{1 / 2}(\mathrm{eV})$

$-v_{i, t h}(\mathrm{~cm} / \mathrm{sec})=10^{6} \mathrm{~T}_{\mathrm{i}}^{1 / 2}(\mathrm{eV})$ 


\title{
Introduction to Plasma Dynamo, Reconnection and Shocks
}

\author{
T. Intrator \\ P-24 Plasma Physics \\ 2012 July 03
}

P-24 Plasma Physics Summer School

Center for Non Linear Studies conference room

Wednesdays $1 \mathrm{pm}-2 \mathrm{pm}$ 


\section{Abstract}

In our plasma universe, most of what we can observe is composed of ionized gas, or plasma. This plasma is a conducting fluid, which advects magnetic fields when it flows. Magnetic structure occurs from the smallest planetary to the largest cosmic scales. We introduce at a basic level some interesting features of non linear magnetohydrodynamics (MHD). For example, in our plasma universe, dynamo creates magnetic fields from gravitationally driven flow energy in an electrically conducting medium, and conversely magnetic reconnection annihilates magnetic field and accelerates particles. Shocks occur when flows move faster than the local velocity (sonic or Alfven speed) for the propagation of information. Both reconnection and shocks can accelerate particles, perhaps to gigantic energies, for example as observed with $10^{20} \mathrm{eV}$ cosmic rays. 


\section{Outline}

- Our magnetic universe

- dynamo

- reconnection

- Shocks

- Summary 


\section{Universal factoids}

- Radius $\approx 46 \times 10^{9} \mathrm{LY} \approx 46 \times 10^{25} \mathrm{~m}$

- Volume $\approx 3.5 \times 10^{80} \mathrm{~m}^{3}$

- Mass $\approx 10^{52} \mathrm{~kg}$ (includes 73\% dark energy, $23 \%$ dark matter, $4 \%$ baryonic matter)

- Mass-energy $=\mathrm{m}_{\text {baryon }} \mathrm{c}^{2} \approx 4 \times 10^{67}$ Joule

- Intracluster B field $\approx 0.1-10 \mu$ Gauss (Kronberg, Pop2003)

- Magnetic energy $(1 \mu \mathrm{G})=\mathrm{B}^{2} / 2 \mu_{0} \times \mathrm{Vol} \approx 2.5 \%$

Light Year $=9.46 \times 10^{15} \mathrm{~m}$, parsec $=3.26 \mathrm{LY}$ 


\section{Solar system confronts interstellar medium}

magnetic bubbles

- Earth

- solar system

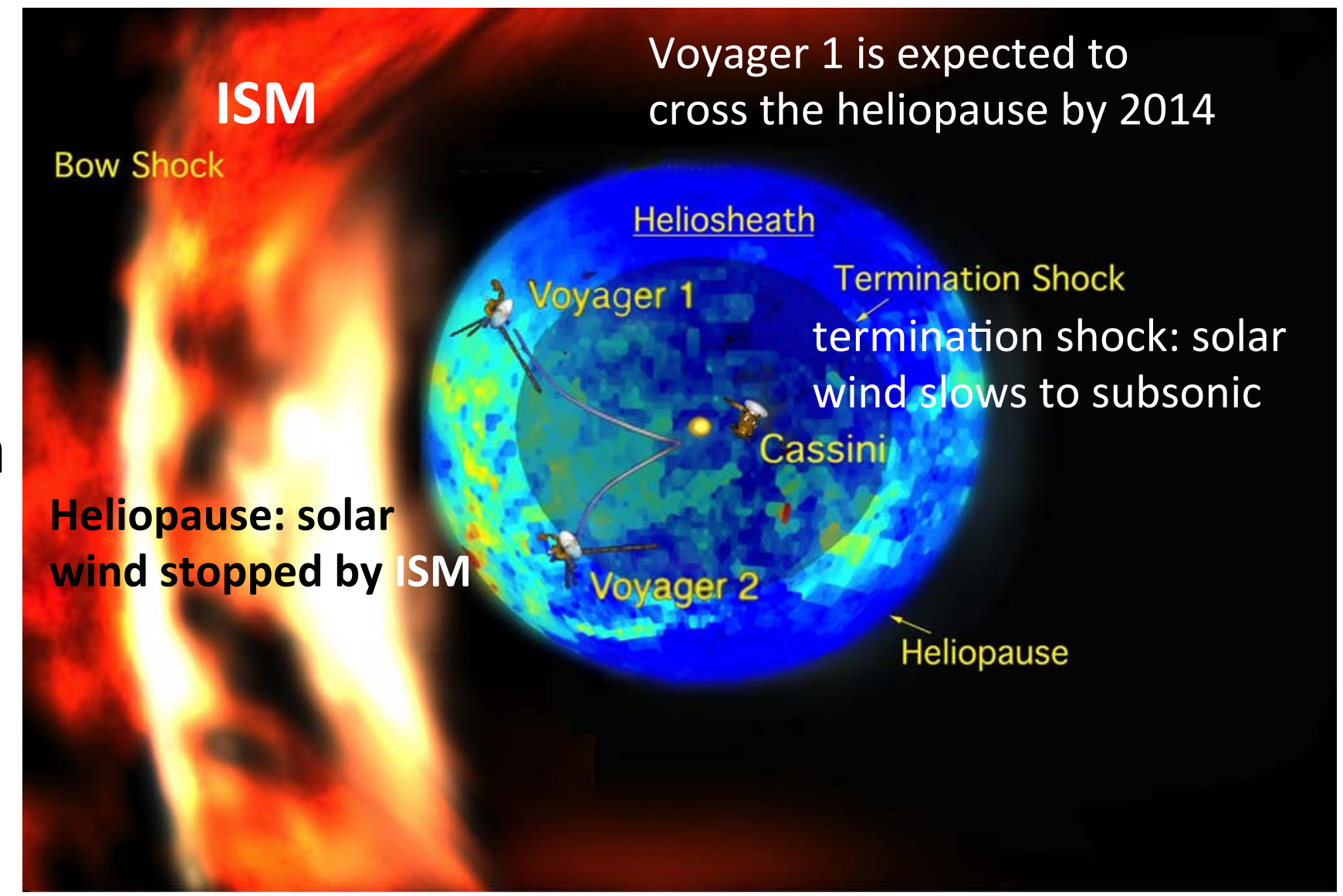

http://www.nasa.gov/images/content/462848main_helioshere_946-710.jpg 


\section{IBEX data}

- Description English: In this illustration, the multicolored (blue and green) bubble represents the new measurements of the emission of particles known as energetic neutral atoms. The energetic neutral atoms were streaming in from the thick boundary known as the heliosheath. The heliosheath is the region between the heliosphere, the region of our sun's influence, and the interstellar medium, the matter between stars in our galaxy. Areas in red indicate the hottest, most high-pressure regions and purple the coolest, lowest-pressure regions.

- The yellow circle is our sun. The two Voyager spacecraft, illustrated with lines showing their path, are currently traveling through the heliosheath. In the heliosheath, the solar wind slows down and heats up as it interacts with the interstellar medium. The image also shows Cassini, which is still inside our solar system, orbiting Saturn. The spacecraft sizes are not to scale.

- The dark inner circle represents the volume bounded by the termination shock, formed where supersonic solar wind streaming out from our sun suddenly slows down. The outer circle, known as the heliopause, the outer boundary of the heliosheath, is the place where the interstellar medium and the solar wind are balanced. To the left of this bubble is the curve of the putative bow shock, where the interstellar medium, traveling in the opposite direction against the heliosheath, slows down as it collides with the heliosphere. The bow shock resembles a wave formed in a stream as it flows around a rock.

- Date 20 November 2009

- Source http://photojournal.jpl.nasa.gov/catalog/PIA12375 , http://en.wikipedia.org/wiki/File:PIA12375.jpg

- $\quad$ Author NASA/JPL/JHUAPL 


\section{Heliospheric current sheet in 3D}

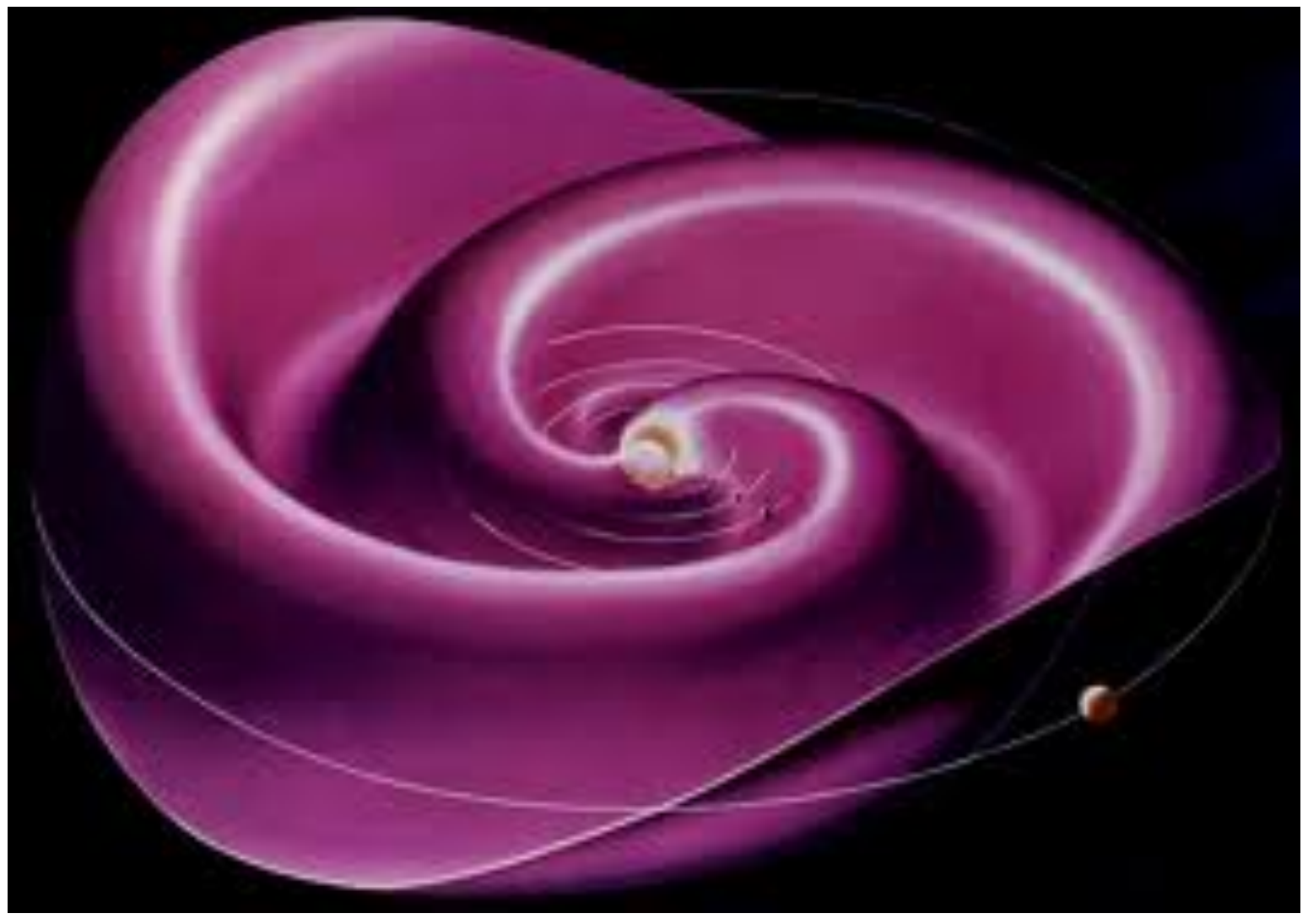

Magnetic field sourced by rotating sun, heliospheric current sheet out to the orbit of Jupiter - "Ballerina skirt" 


\section{High Energy Cosmic Ray Physics}

- The flux of cosmic ray nuclei has to a first approximation, a rapidly falling power law in energy, $\mathrm{dN} / \mathrm{dE} \propto \mathrm{E}^{-\alpha}$, with overall index $\alpha \approx 2.8$.

- The spectrum structure ("knee", "ankle") cannot be explained

- Unknown but probably magnetic particle acceleration mechanisms to such

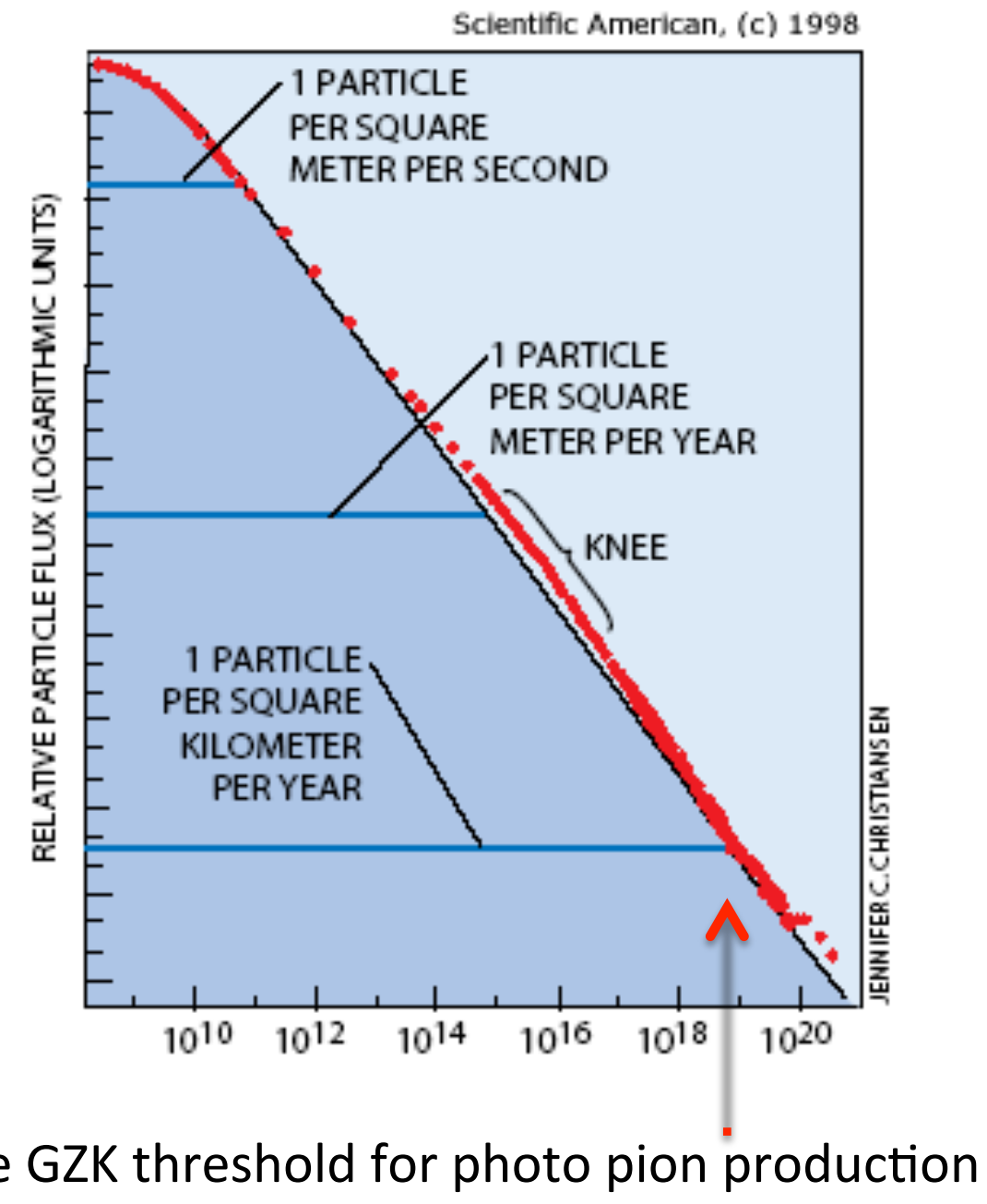
incredible energies 


\section{Magneto Hydro Dynamic approximation}

- If a magnetized plasma has disturbances that are slow enough and large enough spatial scale

- Mobile electrons can prevent the buildup of any electric field that moves with the plasma

- Fields are "stuck" in plasma reference frame - frozen flux theorem

- Higher frequency modes of oscillation and wave propagation do not play a role

- Behavior simplifies substantially

- Magneto Hydro Dynamic (MHD) approximation 


\section{Magnetohydrodynamics: MHD}

- MHD model presumes a single fluid with mass density

$-\rho=\mathrm{n}_{\mathrm{i}} \mathrm{m}_{\mathrm{i}}+\mathrm{n}_{\mathrm{e}} \mathrm{m}_{\mathrm{e}} \approx \mathrm{n}\left(\mathrm{m}_{\mathrm{i}}+\mathrm{m}_{\mathrm{e}}\right) \approx \mathrm{n} \mathrm{m}_{\mathrm{i}}$

- Charge density

- $\sigma=\left(n_{i}-n_{e}\right) e$

- Mass velocity

- $\mathbf{U}=\left(\mathrm{n}_{\mathrm{i}} \mathrm{m}_{\mathrm{i}} \mathbf{u}_{\mathrm{i}}+\mathrm{n}_{\mathrm{e}} \mathrm{m}_{\mathrm{e}} \mathbf{u}_{\mathrm{e}}\right) / \rho \approx \mathrm{u}_{\mathrm{i}}+\left(\mathrm{m}_{\mathrm{e}} / \mathrm{m}_{\mathrm{i}}\right) \mathbf{u}_{\mathrm{e}}$

- Current density

- $\mathrm{J}=\mathrm{e}\left(\mathrm{n}_{\mathrm{i}} \mathrm{u}_{\mathrm{i}}-\mathrm{n}_{\mathrm{e}} \mathrm{u}_{\mathrm{e}}\right) \approx \mathrm{ne}\left(\mathrm{u}_{\mathrm{i}}-\mathrm{u}_{\mathrm{e}}\right)$

- Single fluid equation of motion

- $\rho \mathrm{du} / \mathrm{dt}=\{\partial \mathrm{u} / \partial \mathrm{t}+\mathrm{u} \bullet \nabla \mathrm{u}\}=\sigma \mathrm{E}+\mathrm{jxB}-\nabla \mathrm{p}$

- Resistive MHD includes finite resistivity

- $E+u \times B=\eta J+\{j \times B-\nabla p\} / e n$ 


\section{Magnetic double helix}

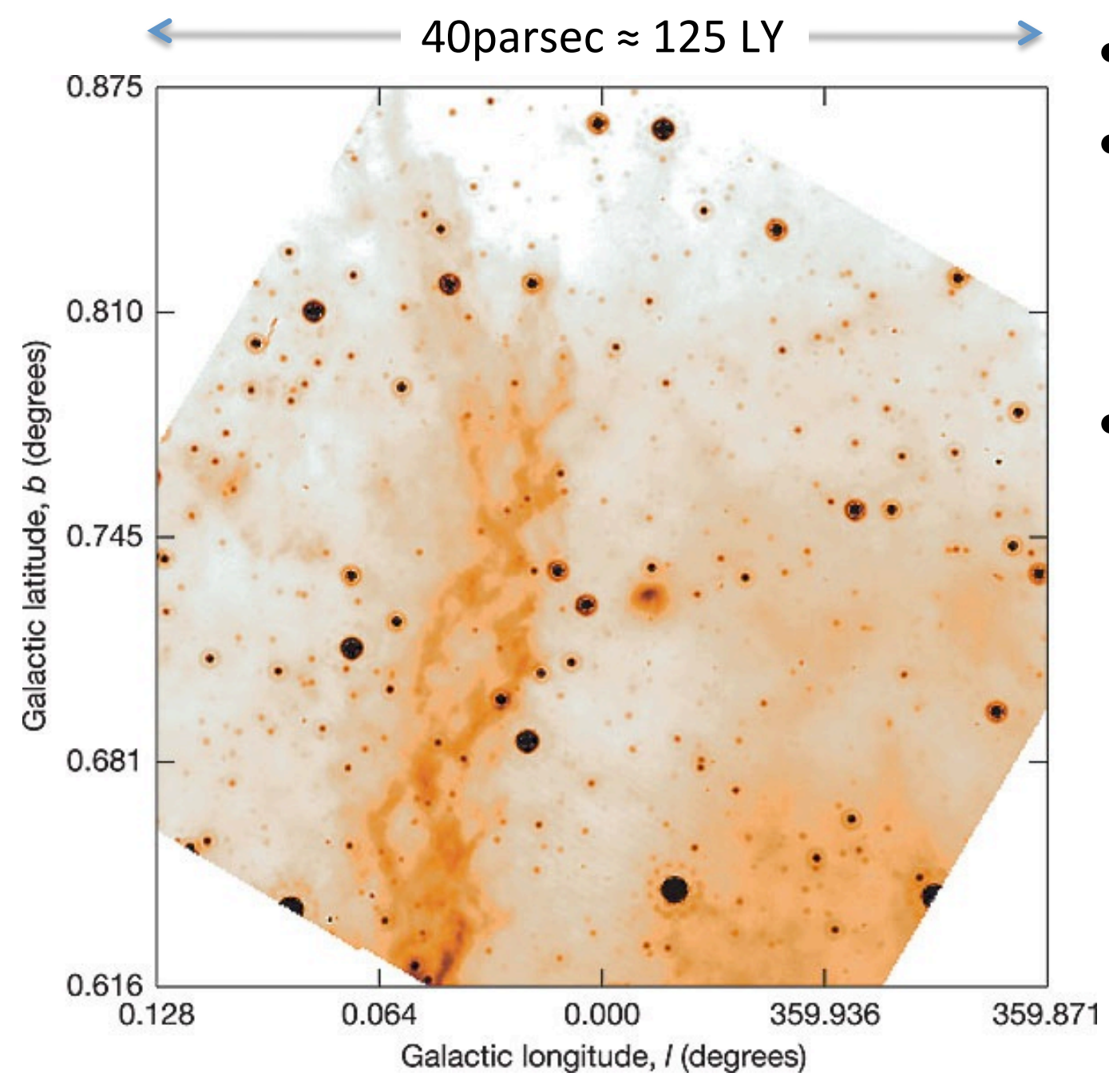

- Double helix nebula

- $\lambda=24 \mu \mathrm{m}$, MIPS

camera, Spitzer Space Telescope

- Near galactic center (8 kpc away)

$\star 6$ arcsec resolution

$\star 1$ arcmin $\Leftrightarrow 2.5 \mathrm{pc}$

$\star$ Parsec $=3.24 \mathrm{LY}$

A magnetic torsional wave near the Galactic Centre traced by a 'double helix' nebula, M Morris, et al, Vol 440|16 March 2006| doi:10.1038/nature04554 


\section{Dynamo}

- The creation of magnetic field from flow in a conducting medium

- Large scale coherent flows

- Random and turbulent motions

- Twist up field lines that are trapped in flow

- Creation of B field from scratch (no one knows how this happens) 


\section{Astrophysical Dynamo: The Origin of Large Scale, Coherent Magnetic Fields}

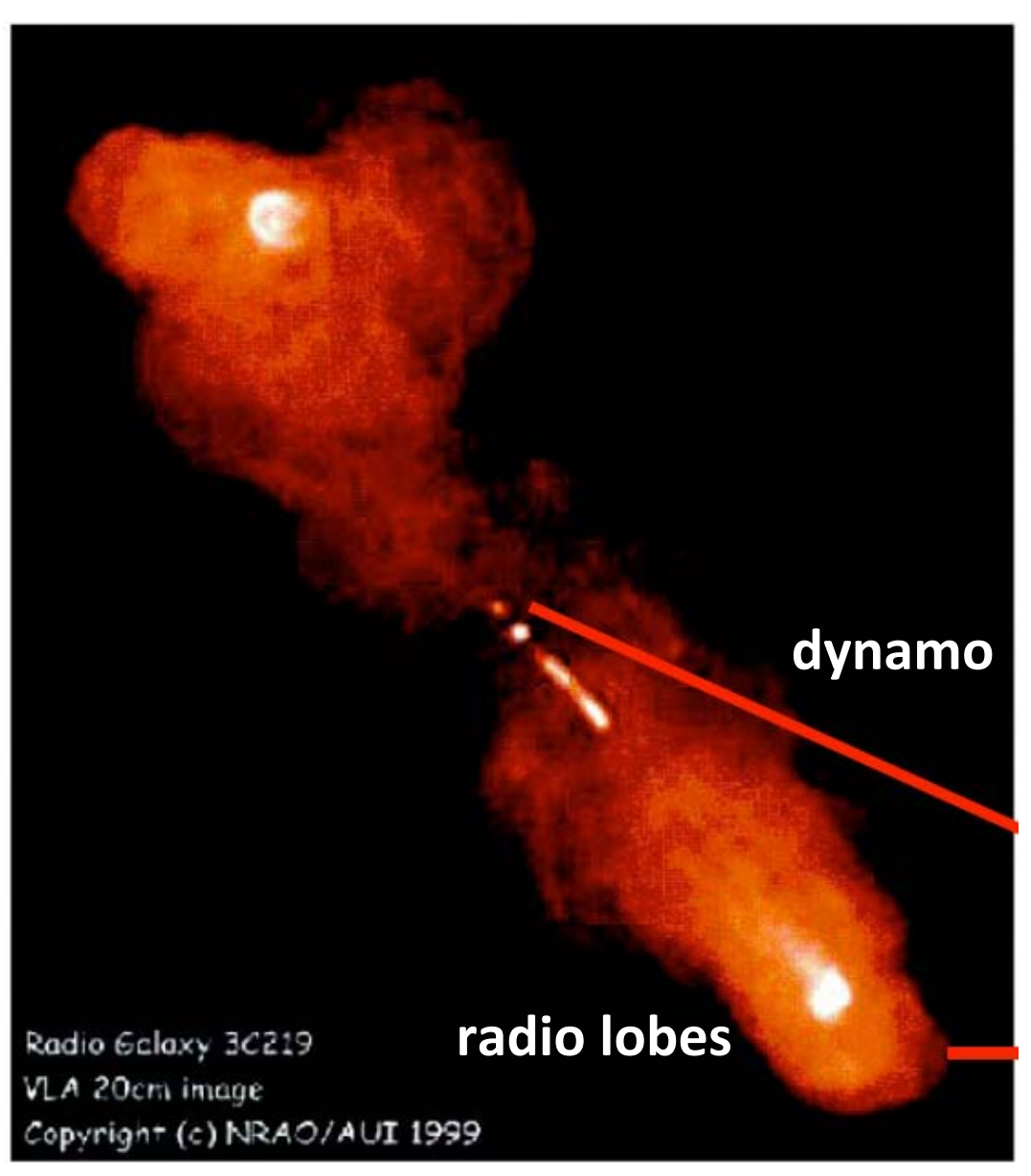

S. Colgate
- 3C219 Radio Lobe, - 1\% of $\mathrm{MBH} \mathrm{c} \mathrm{c}^{2}$ in $10^{8}$ years.

- Not clear if it is the random motions + fluid turbulence, or large scale coherent motions

- Massive black hole accretion disk dynamo is small $(\approx$ $\left.30 \mathrm{r}_{\mathrm{Gi}} \approx 10^{15} \mathrm{~cm}\right) \approx 10^{-10}$ of the radio lobe $\left(\sim 3 \times 10^{24} \mathrm{~cm}\right)$ Frequency $10^{10} \mathrm{~Hz}$

- $\mathrm{L}_{\text {lobe }} \sim 10^{38}-10^{39}$ Watt 


\section{Astrophysical Dynamo: The Origin of Large Scale, Coherent Magnetic Fields}

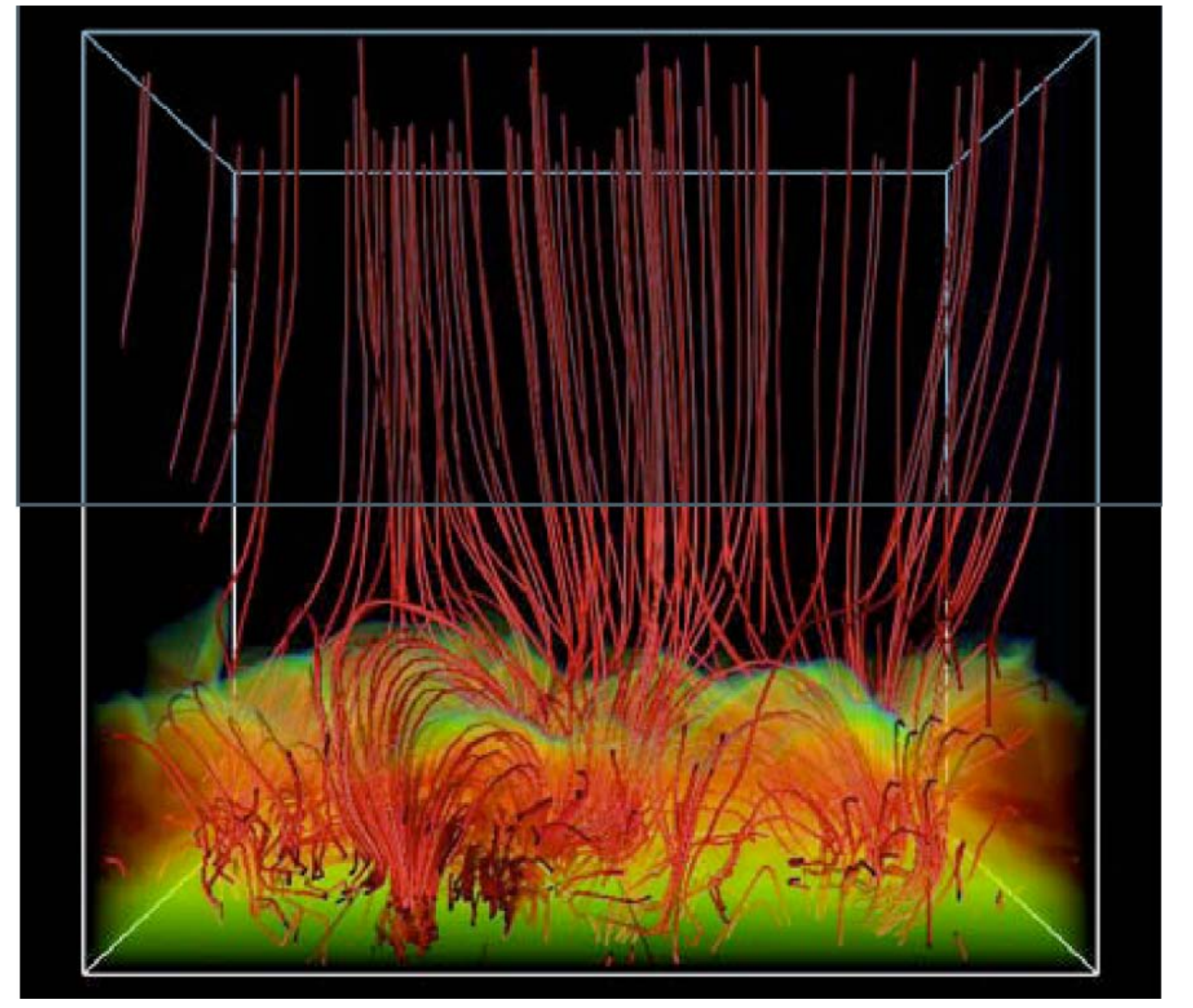

- B fields on the sun

- simulation, $10^{4} \mathrm{x}$ granulation scale

- Convective plumes base of convective zone 


\section{$\alpha-\Omega$ dynamo}

- Keplerian sheared galaxy rotation (the $\Omega$ part)

- Balance centripetal with gravitational force

$-V_{\phi}^{2} / r=G M / r^{2}$

$-V_{\phi}(r)=(G M / r)^{1 / 2}$

Sheared motion winds up magnetic $B_{z}$ lines ( $\alpha$ shaped) outside the accretion disk.

$\Rightarrow$ Toroidal B field

$\Rightarrow$ force-free helix

$\Rightarrow$ magnetic flux of the universe??

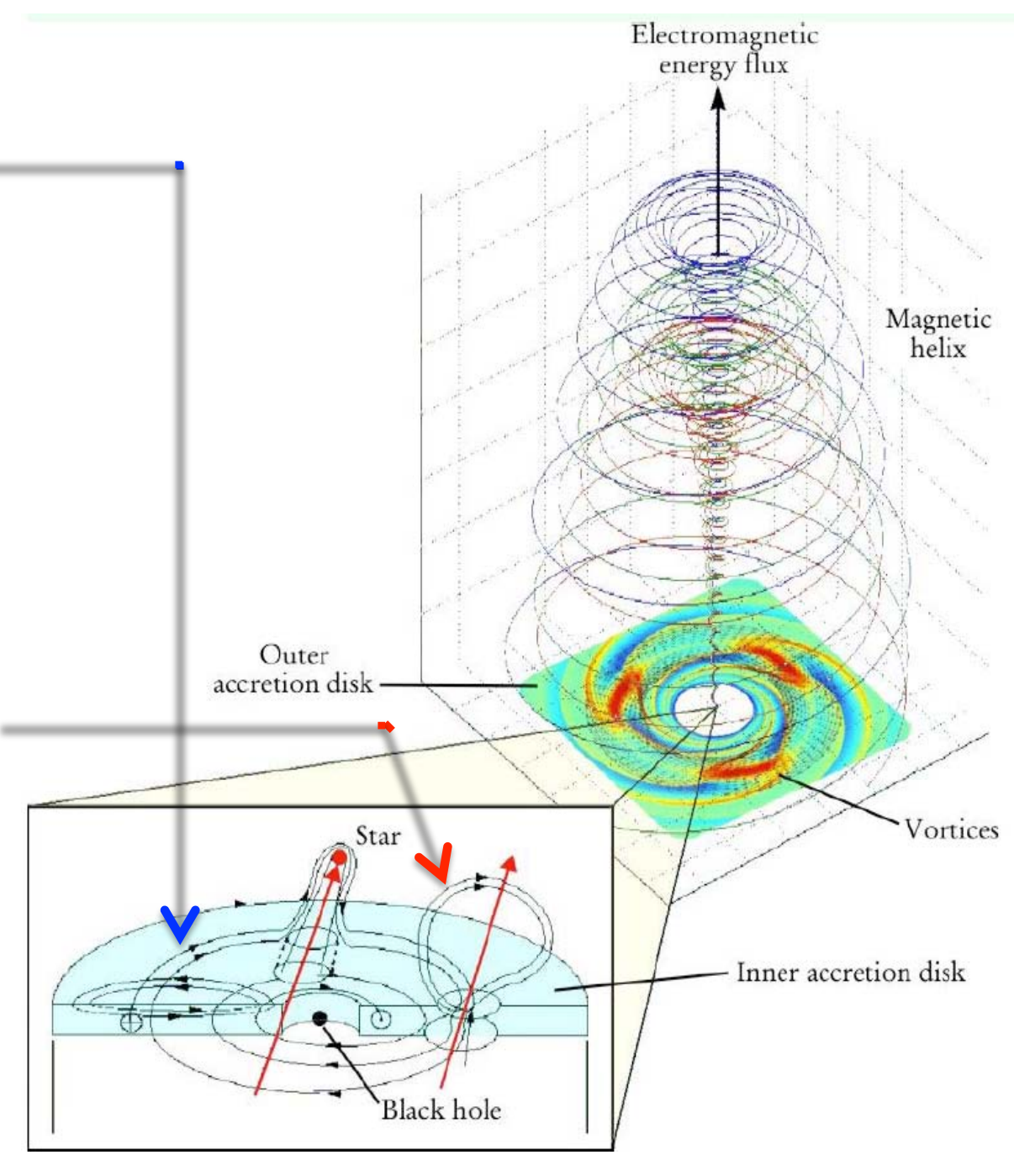




\section{Accretion disc, black hole rotating, shock emission}

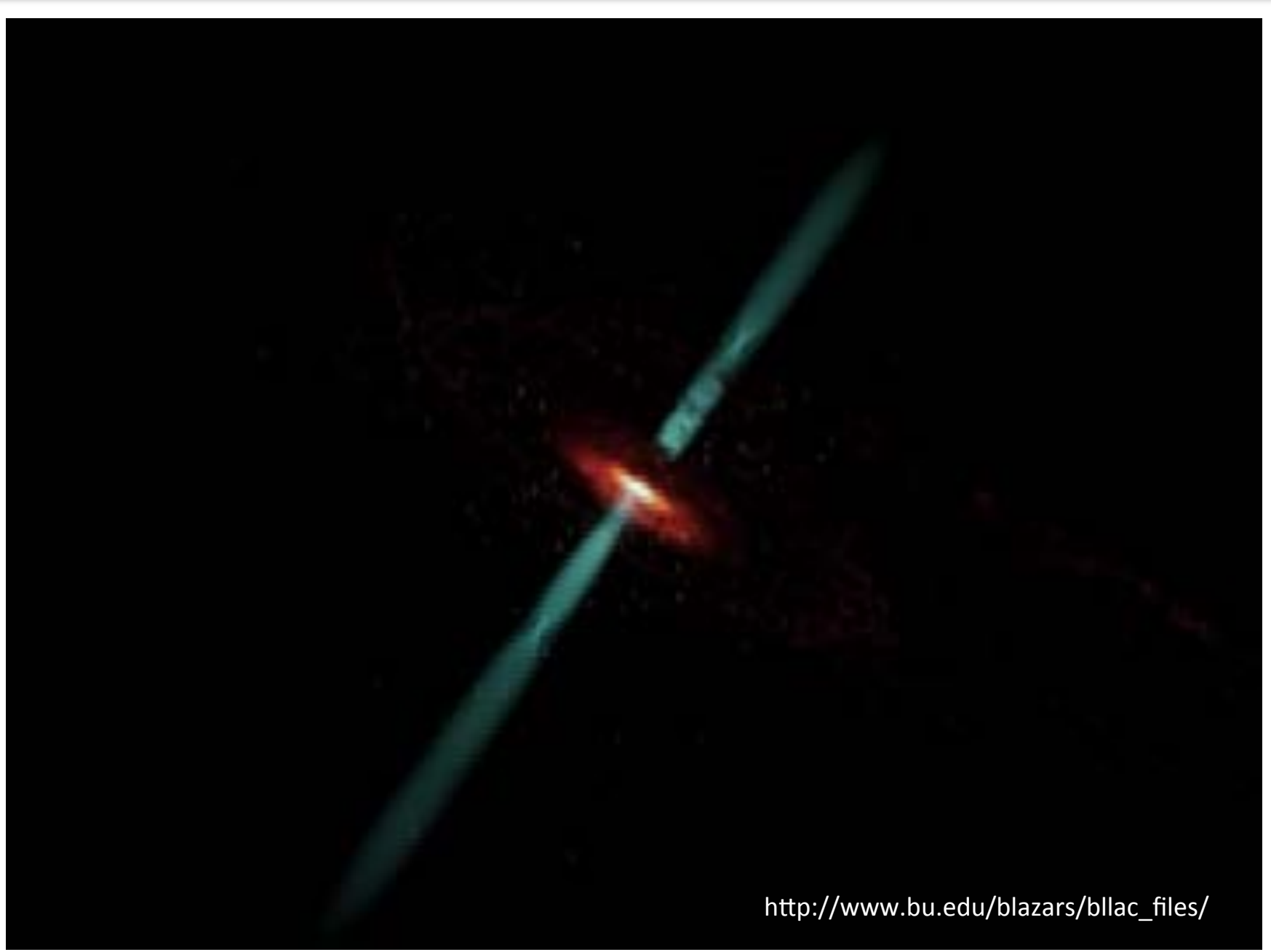




\section{Integral Maxwell equations}

\section{Maxwell's Equations}

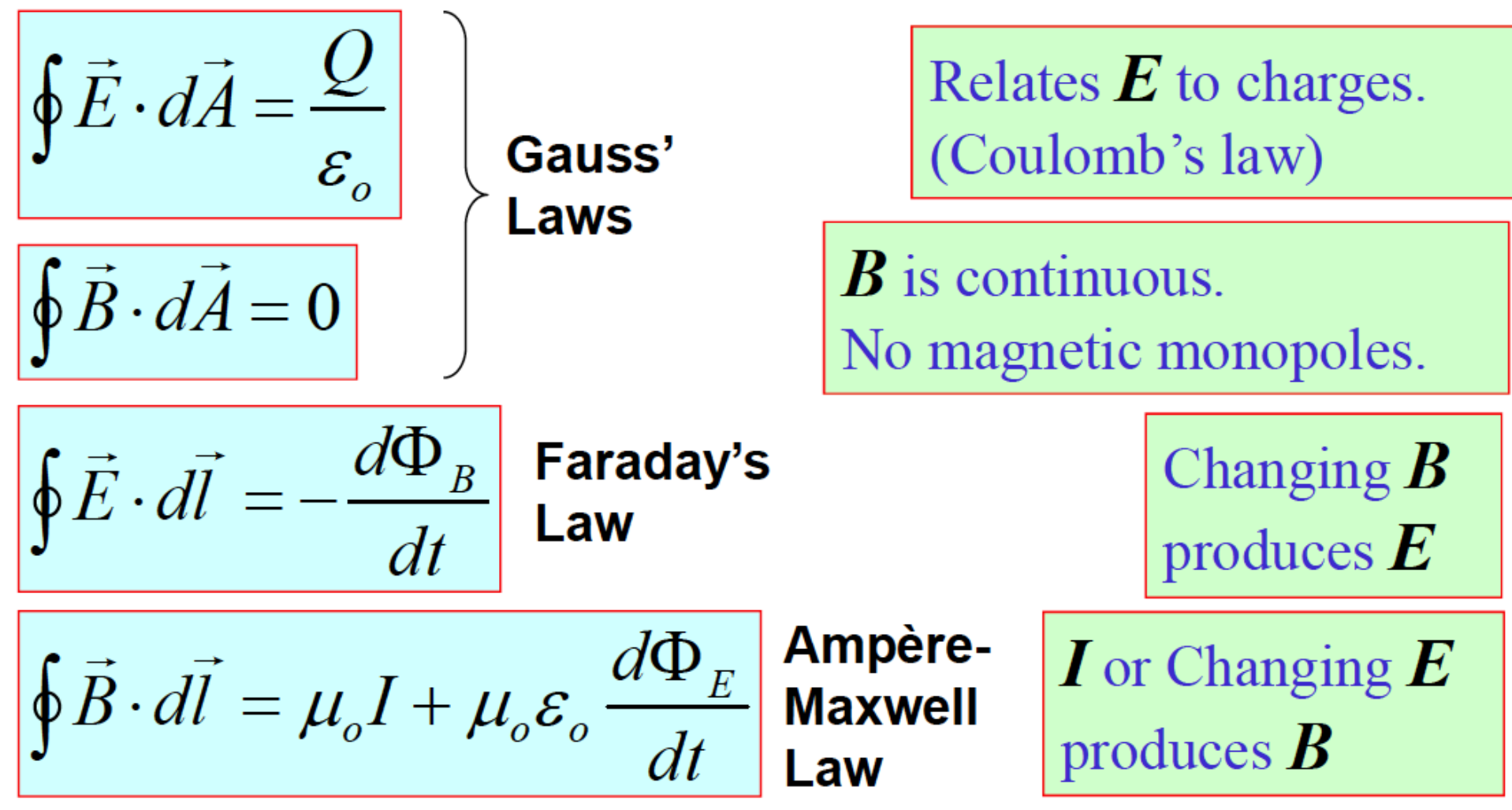

These equations describe all electric \& magnetic phenomena (in the absence of dielectric or magnetic materials). 


\section{Magnetic field from fluctuations}

- Newton's law F=ma↔momentum balance

$-\partial(\rho v) / \partial t=E+v x B-\nabla p_{e}+J x B$

- Ohms law includes collisions (resistivity $\eta$ )

$-E+v x B=J x B /(e n)-\nabla p_{e} /(e n)+\eta J$

- Recall from Maxwell equations $\nabla x E=-\partial B / \partial t$

- Suppose fields have mean + fluctuations

$-\mathrm{B}=\mathrm{B}_{0}+\delta \mathrm{B}, \mathrm{E}=\mathrm{E}_{0}+\delta \mathrm{E}, \mathrm{v}=\mathrm{v}_{0}+\delta \mathrm{v}, \ldots$ etc

$-\nabla x\langle\delta E\rangle=-\nabla x\langle\delta v x \delta B\rangle+\nabla x\langle\delta J x \delta B\rangle+\ldots$

- $\rightarrow \delta \mathrm{B}$ grows from correlated fluctuations 


\section{Magnetic reconnection}

- Magnetic field lines and fluxes are "frozen" into fluid flow $(E+v \times B=0)$

- Fluxes are advected towards each other by colliding flows

- Oppositely directed magnetic fields smash together and annihilate (beyond ideal MHD)

- Magnetic field annihilation energy energizes particle velocities 


\section{Reconnection animation}

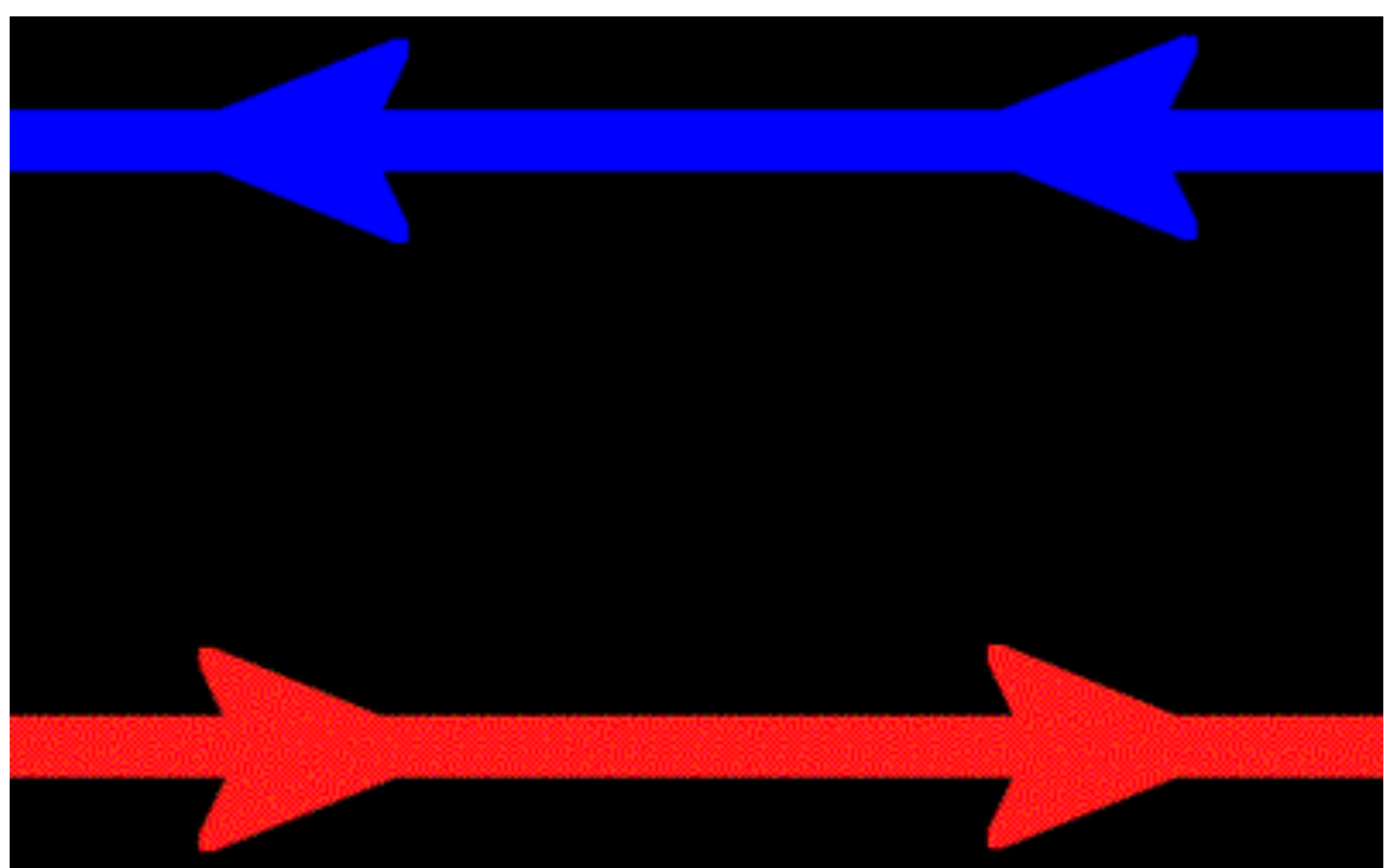

NASA, CLUSTER team 


\section{Filamented Plasma Structure in the Universe}
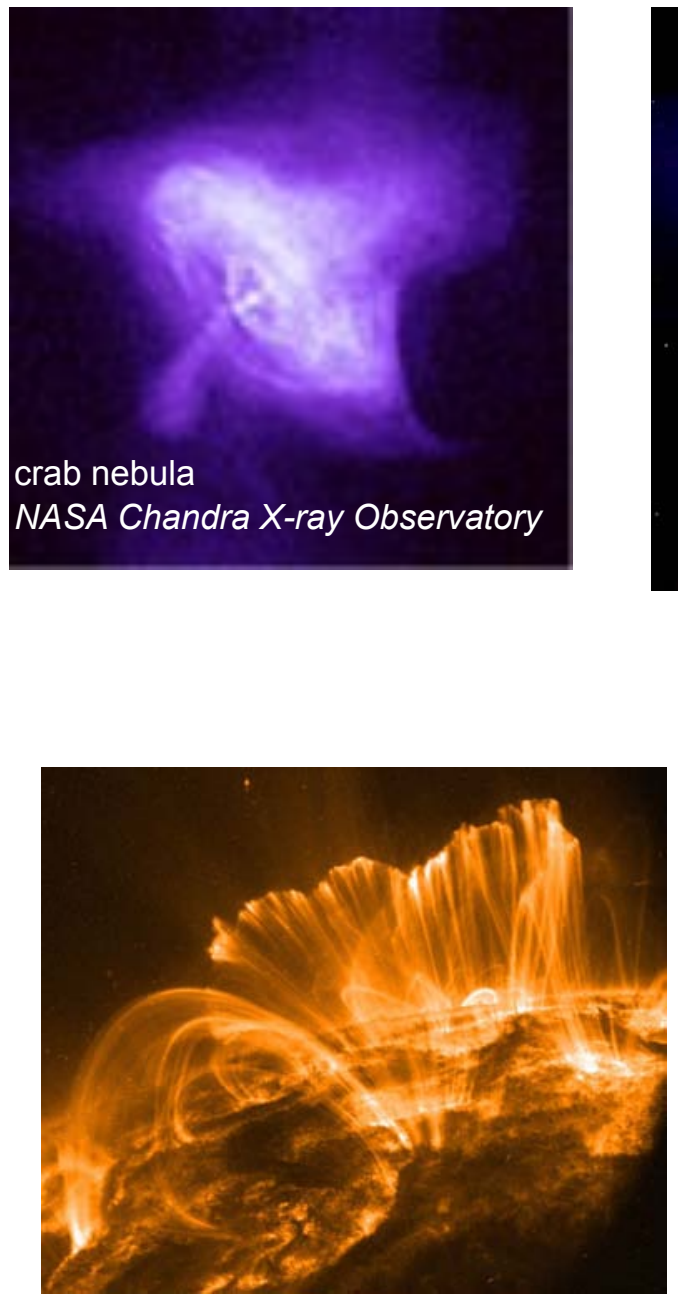

TRACE satellite VUV images

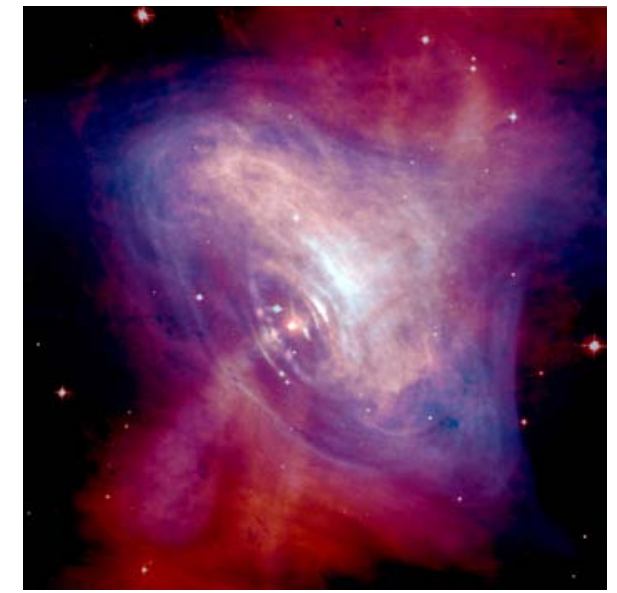

Crab Pulsar. This image combines optical data from Hubble (in red) and Xray images from Chandra $X$-ray

Observatory (in blue)

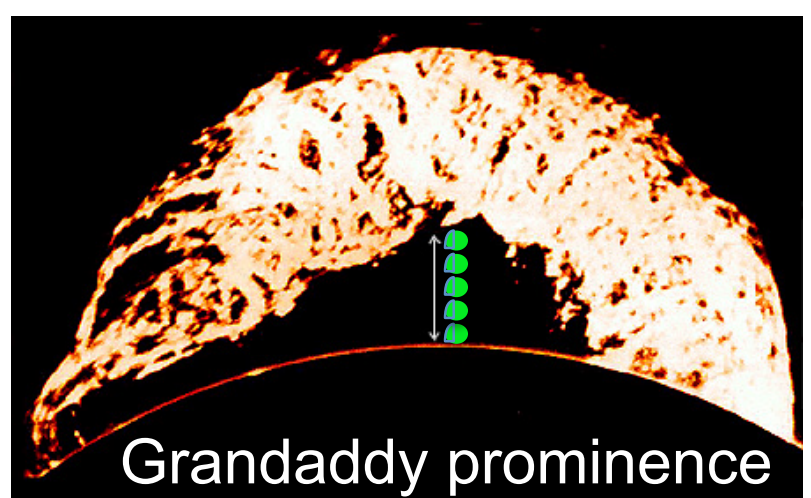

Arrow is $64,000 \mathrm{~km}, 5$ earths high, earth diameter $=$ $12756 \mathrm{~km}$

June 4, 1946 High Altitude Observatory... http://solar-center.stanford.edu/compare

Coronal arches, magnetic structures 


\section{Fields and currents coexist: 3D relaxation}

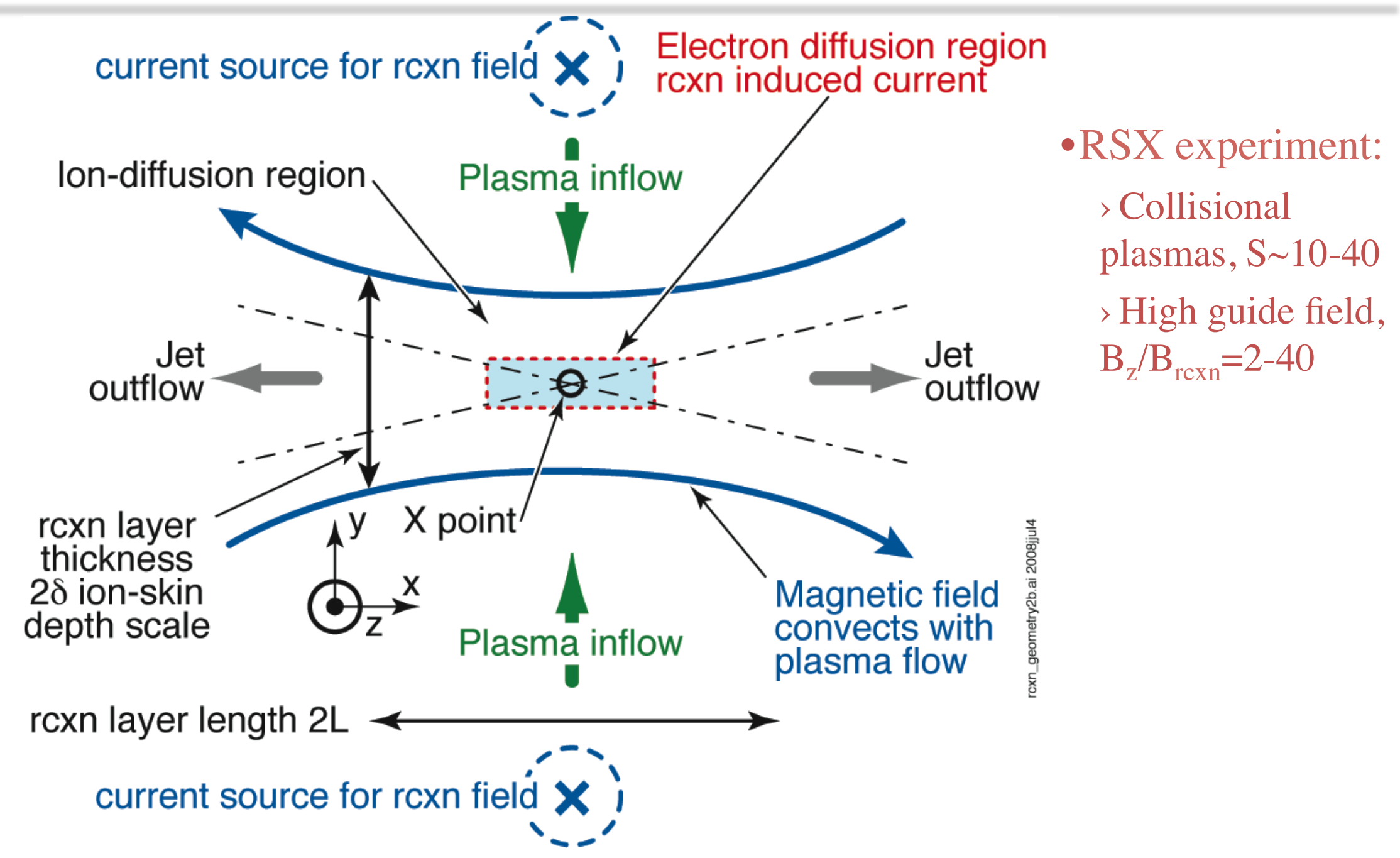




\section{THEMIS view: reconnection \& aurora}

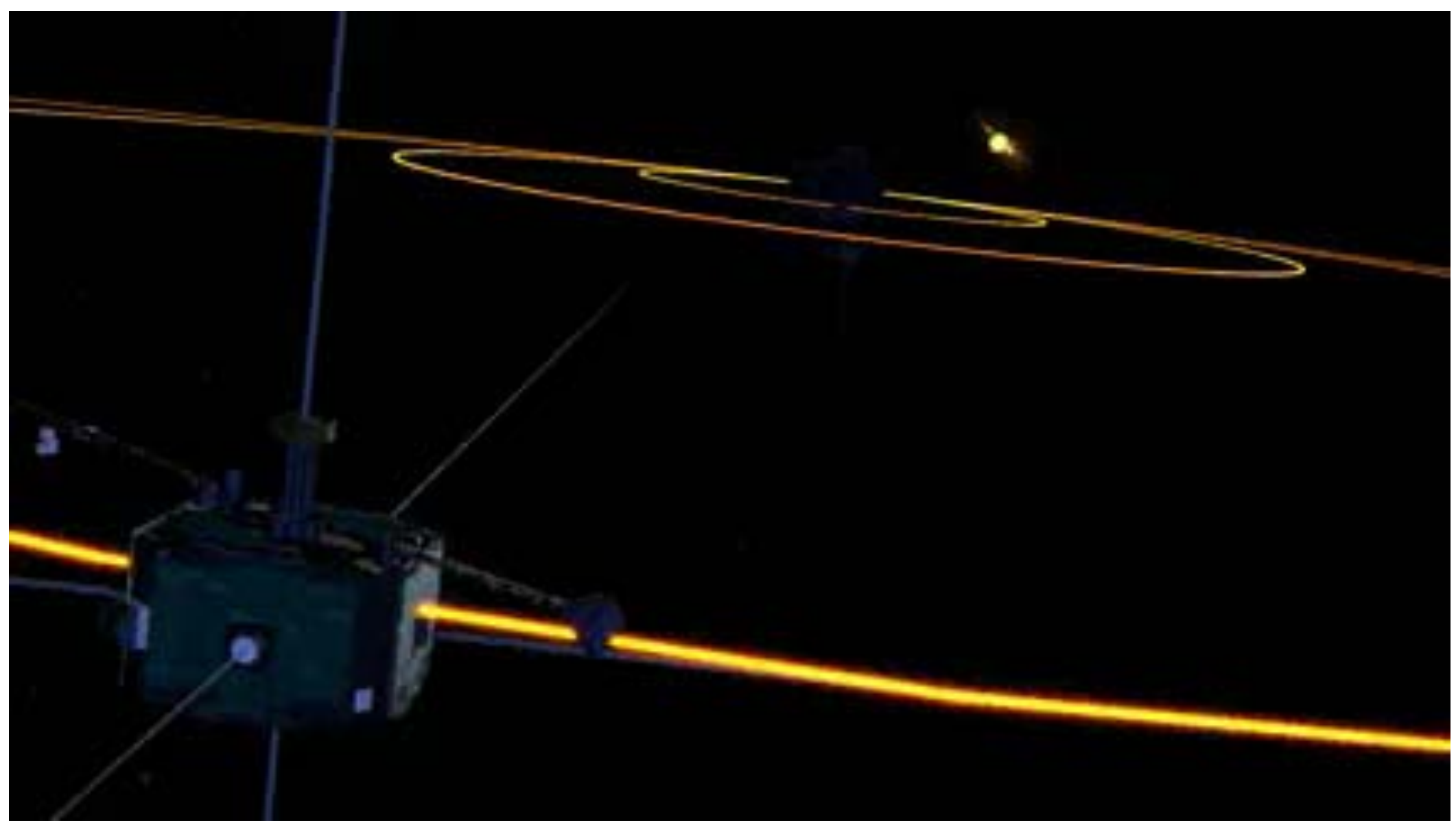




\section{Fast solar wind from coronal holes}

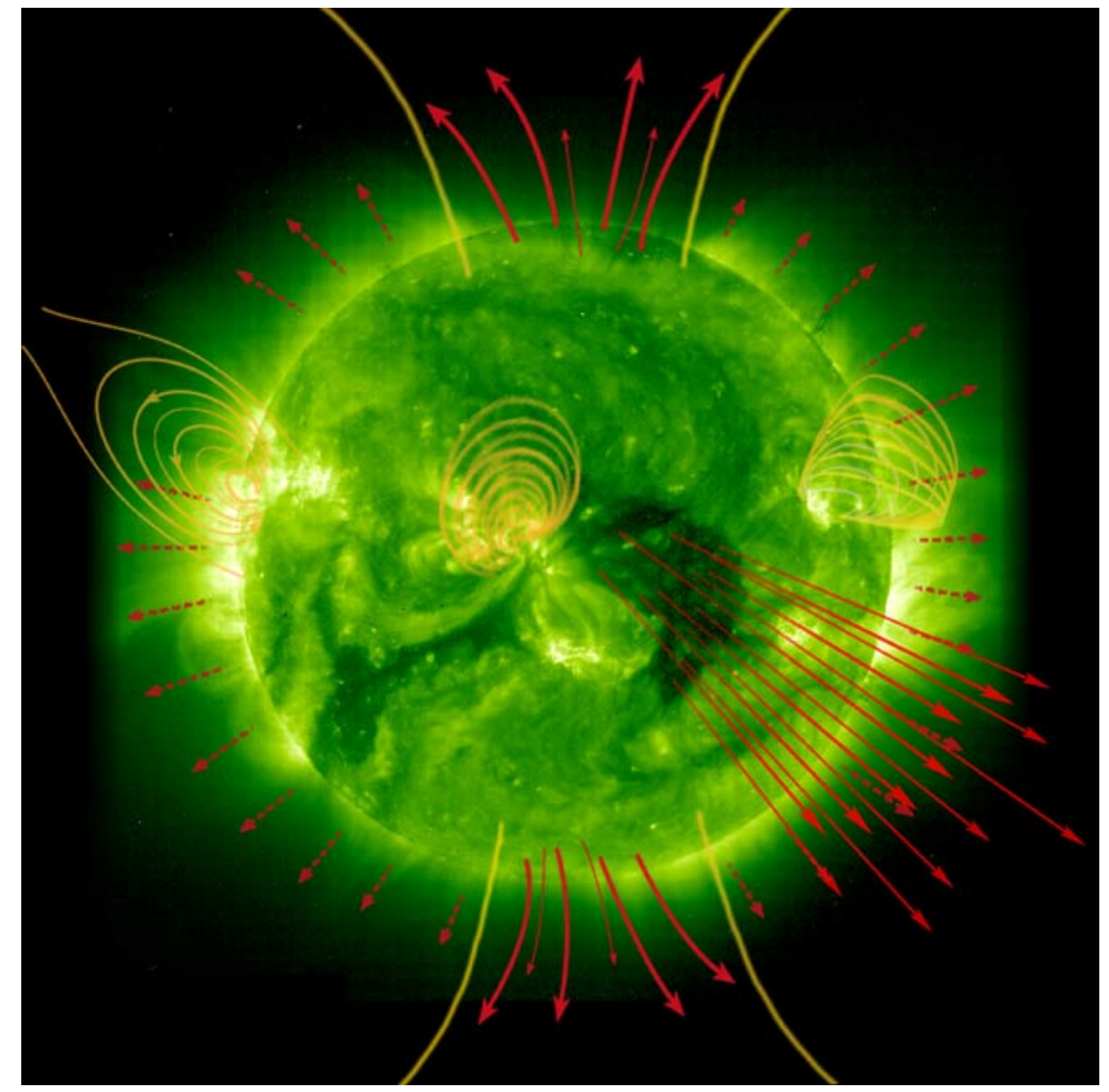

NASA STEREO EUV image
- Red arrows $\rightarrow$ direction of fast solar wind streams leaving the sun.

- Yellow $\rightarrow$ magnetic fields

- Fast solar wind anchored at coronal hole, but the other end winds out to Earth, Mars, Jupiter (magnetic clouds)

- Current + field => Flux ropes! 


\section{Zoom in on coronal holes: flux ropes}
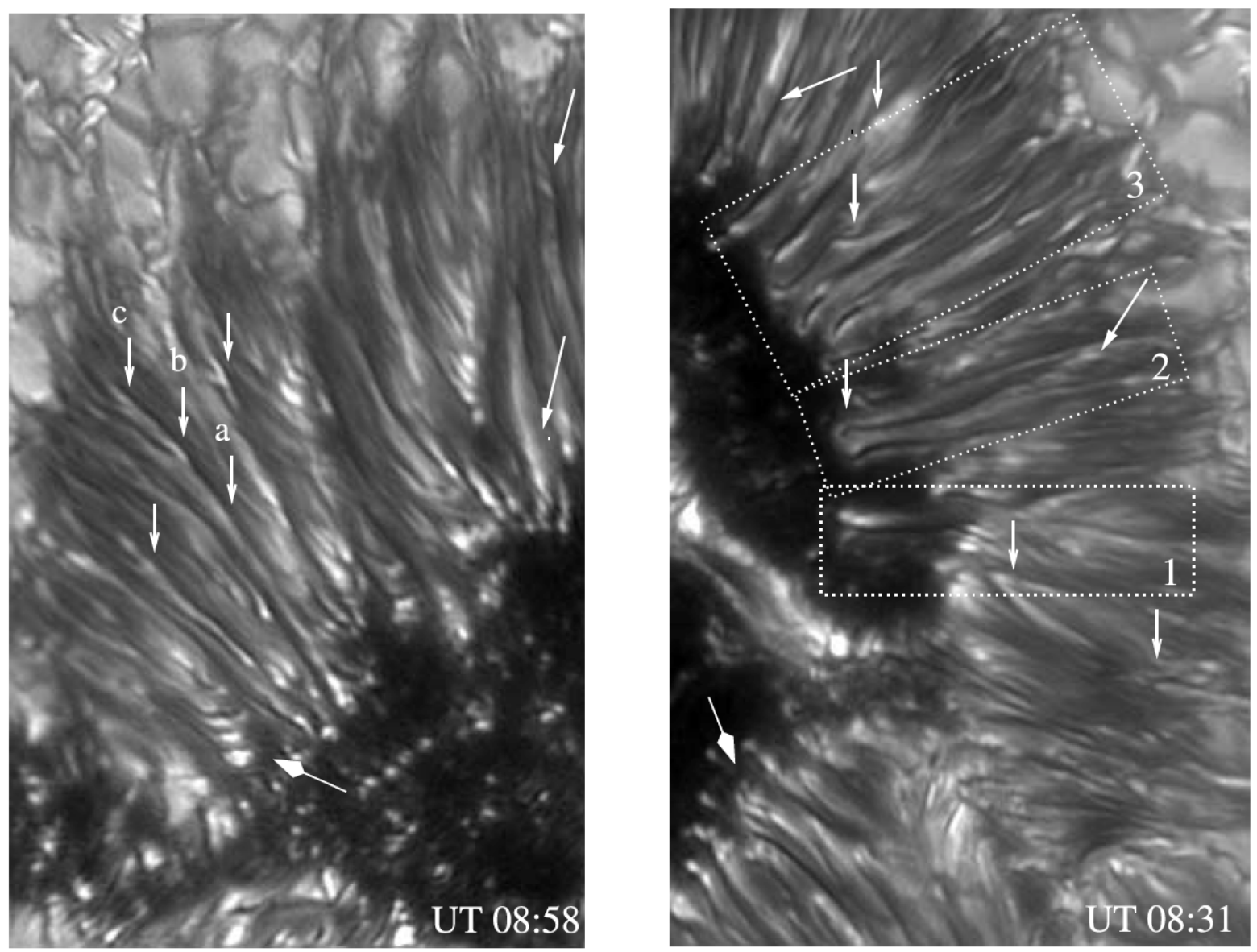

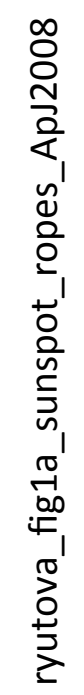




\section{Current ropes modeled in the laboratory}

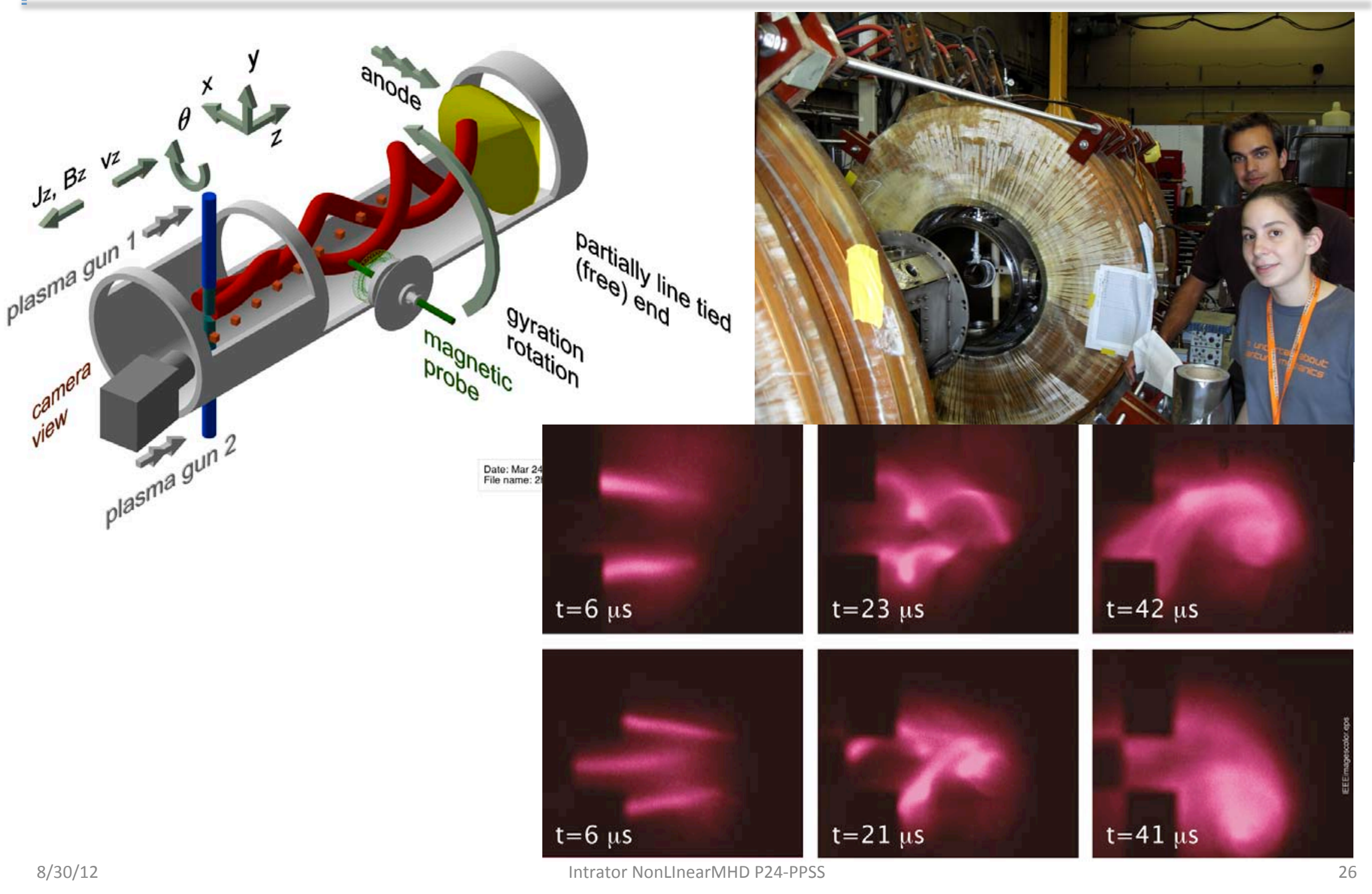




\section{Reconnection and pileup when $v($ inflow $)>v($ Sweet Parker $)$}
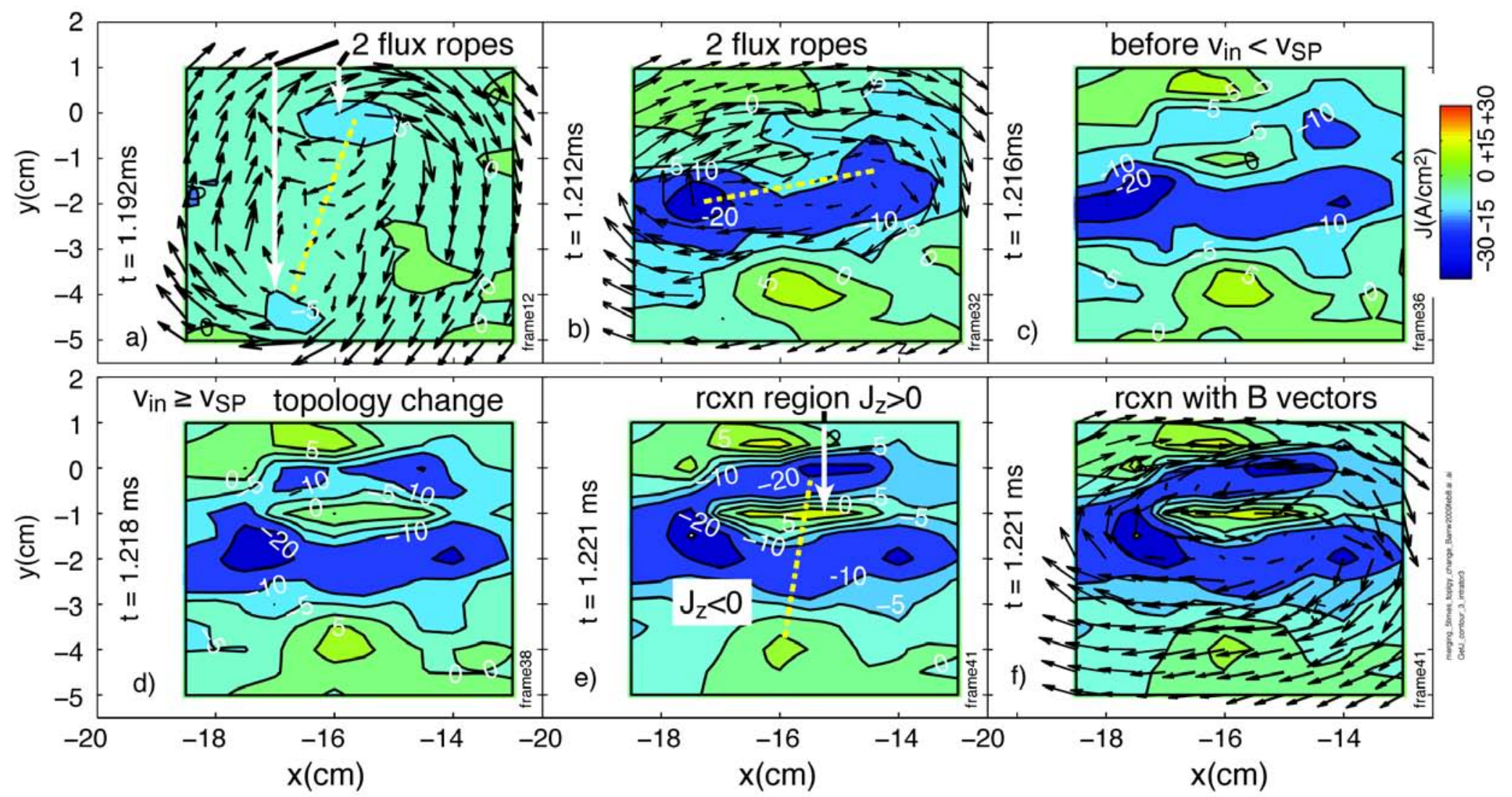

$\frac{B(\text { guide })}{B(\text { rcxn })}=B_{z} / B_{\theta} \approx 10$

Vector $\mathrm{B}(\mathrm{x}, \mathrm{y})$ \& Jz contours

$\mathrm{B}=100$ Gauss, V_bias $=120$ volt 


\section{3 flux ropes in LAPD}

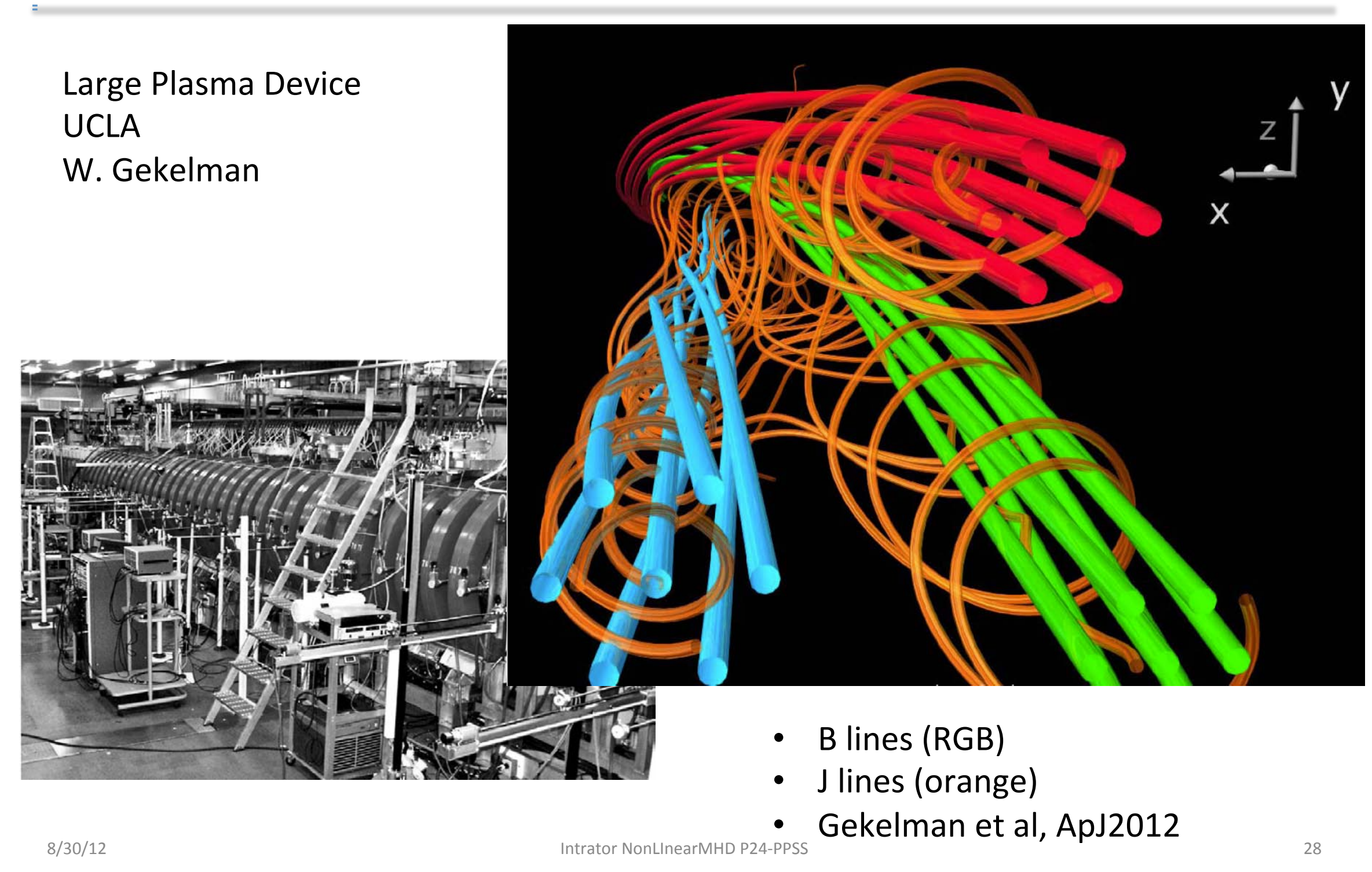




\section{D Reconnection current sheet is intrinsically unstable to formation of islands, plasmoids, flux ropes}




\section{Shocks}

- Shock moves faster than the local information speed

- Fluid near the disturbance cannot react before the disturbance arrives

- Jump in fluid properties (density, pressure, temperature, velocity, Mach number

- decelerates supersonic flow

- Sites of particle acceleration

- Thickness mean free path

- in air: mean free path micron

- On Earth, most shocks are mediated by collisions

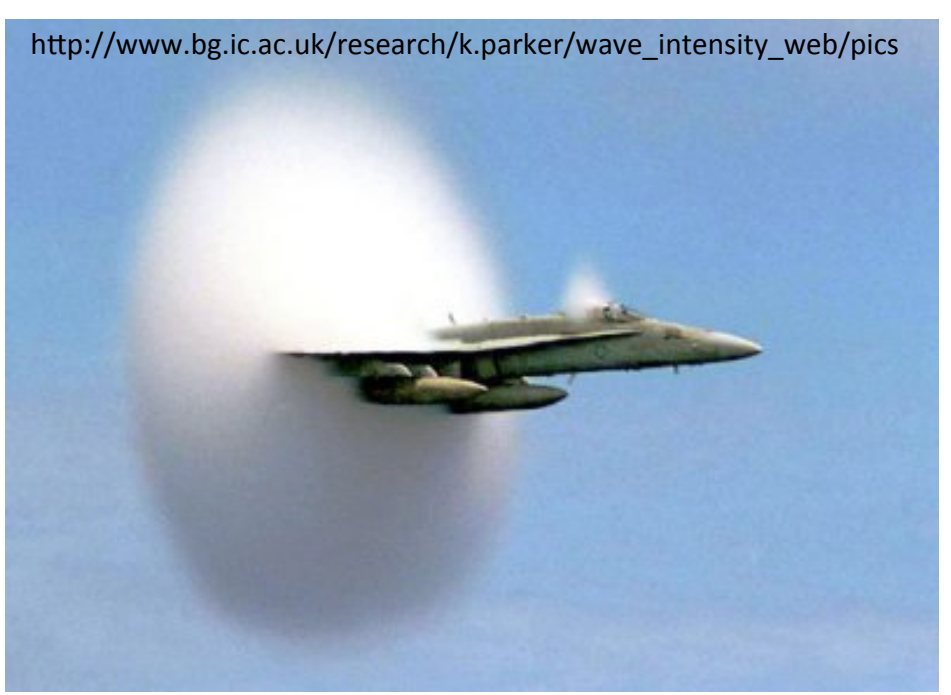

A plane moving faster than the speed of sound creates shock waves that form a cone around the plane. This fighter plane is flying at supersonic speed at low altitude over an ocean in humid conditions. The compression caused by the conical shock waves around the plane causes the water vapour to condense forming the cloud over the rear of the plane, thereby making the effect of the shock waves visible. Cloud is moving with the plane. 


\section{Shock geometry}

\section{Laboratory vs shock reference frame}

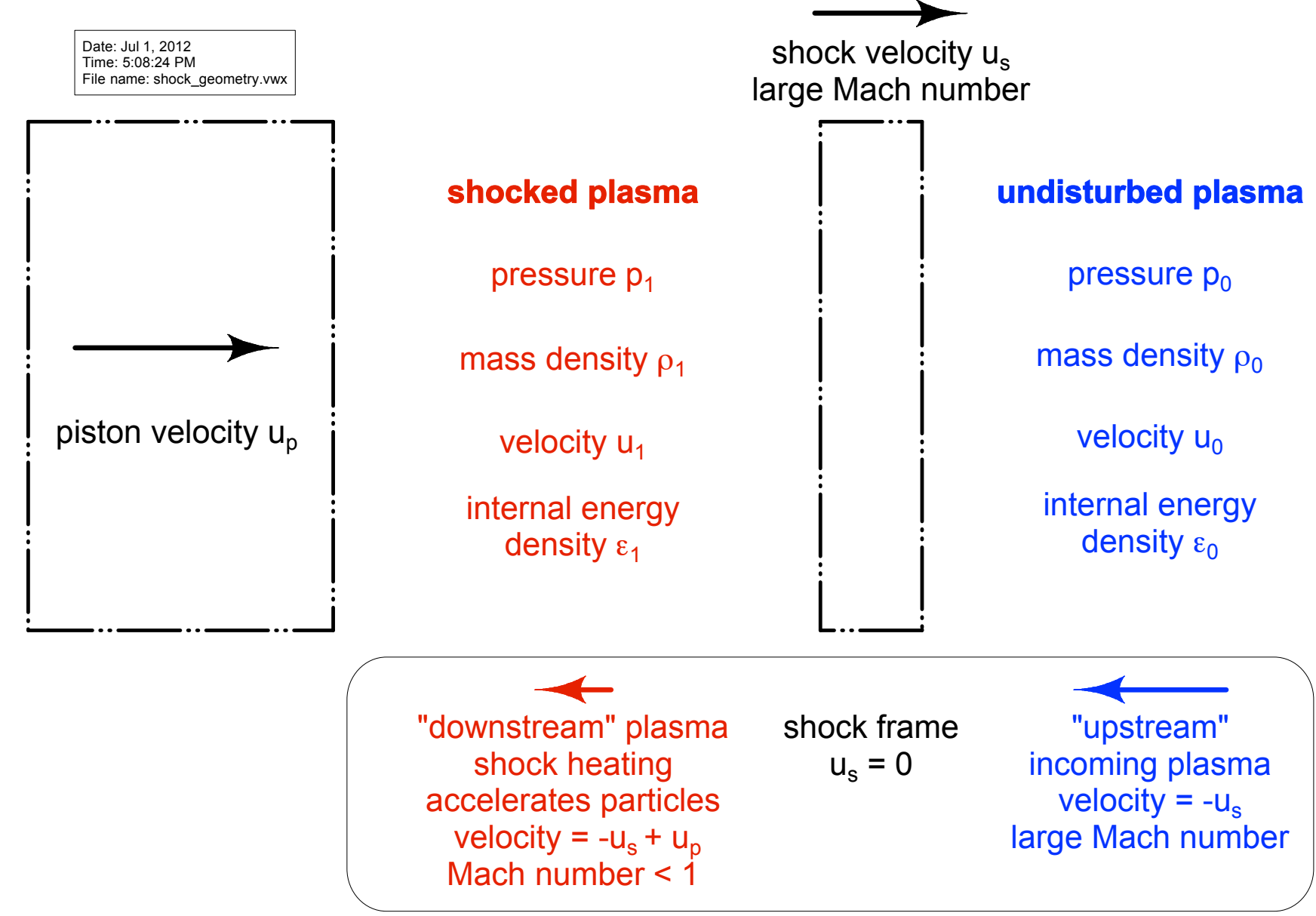




\section{Physics of collisionless shocks}

- Shocks are sites of particle acceleration

- shocks must be mediated without direct collision, but

- Shock: sudden change in density, temperature, pressure that decelerates supersonic flow through interaction with collective fields

- collisionless shocks

- Thickness mean free path

- Astrophysical settings: Mean free path to Coulomb collisions is enormous: $100 \mathrm{pc}$ in supernova remnants, $\sim \mathrm{Mpc}$ in galaxy clusters

- Mean free path > scales of interest 
Earth + solar wind + dipole magnetic field => convecting field lines, bowshock, magnetotail, plasmoid

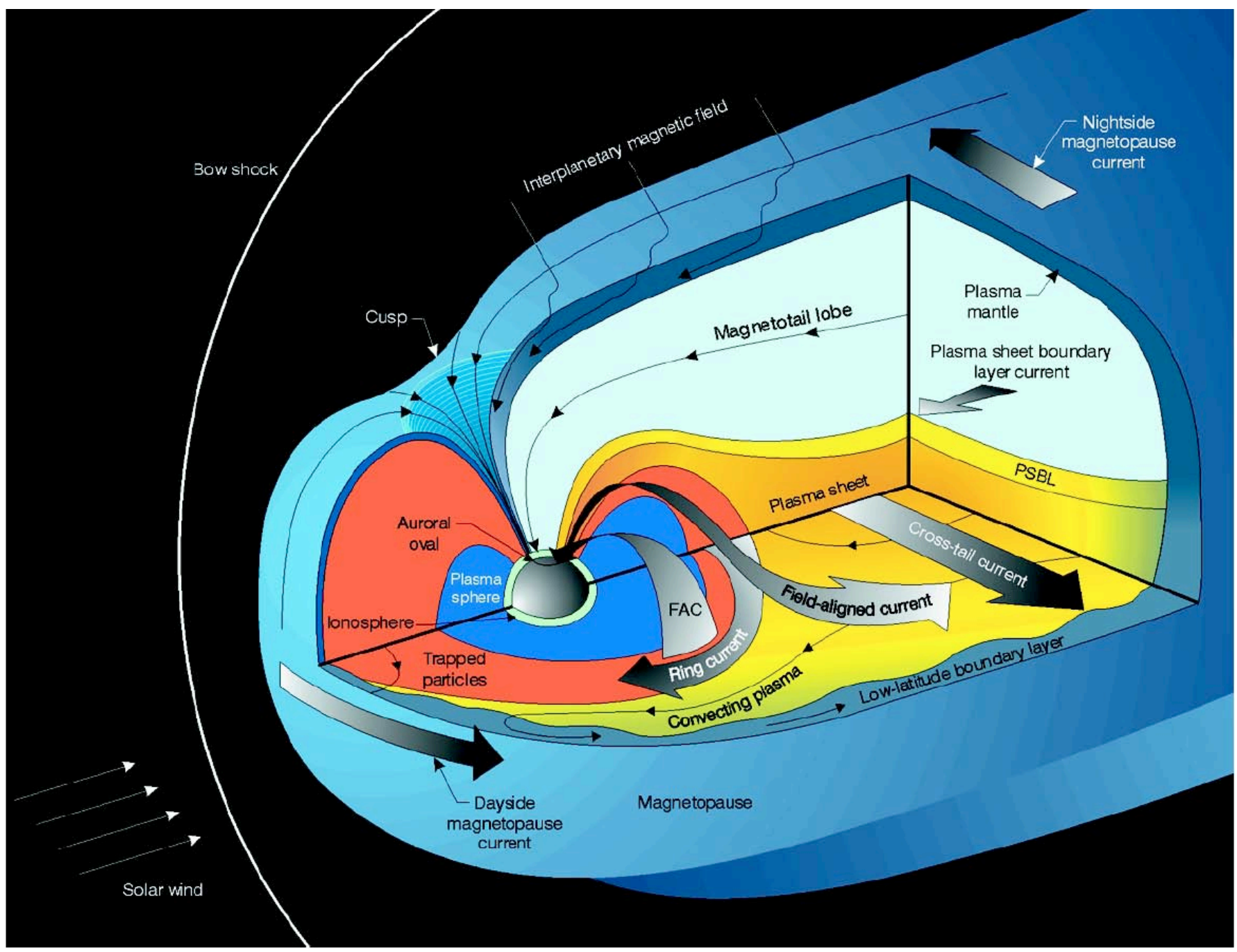




\section{Supernova remnants show shock boundaries}

SNR results from the explosion of a star in a supernova, bounded by an expanding shock wave, and consists of ejected material expanding from the explosion, and the interstellar material it sweeps up and shocks along the way

Ejecta velocities $<10 \%$ the speed of light, highly supersonic, $\mathrm{M}_{\mathrm{S}}$

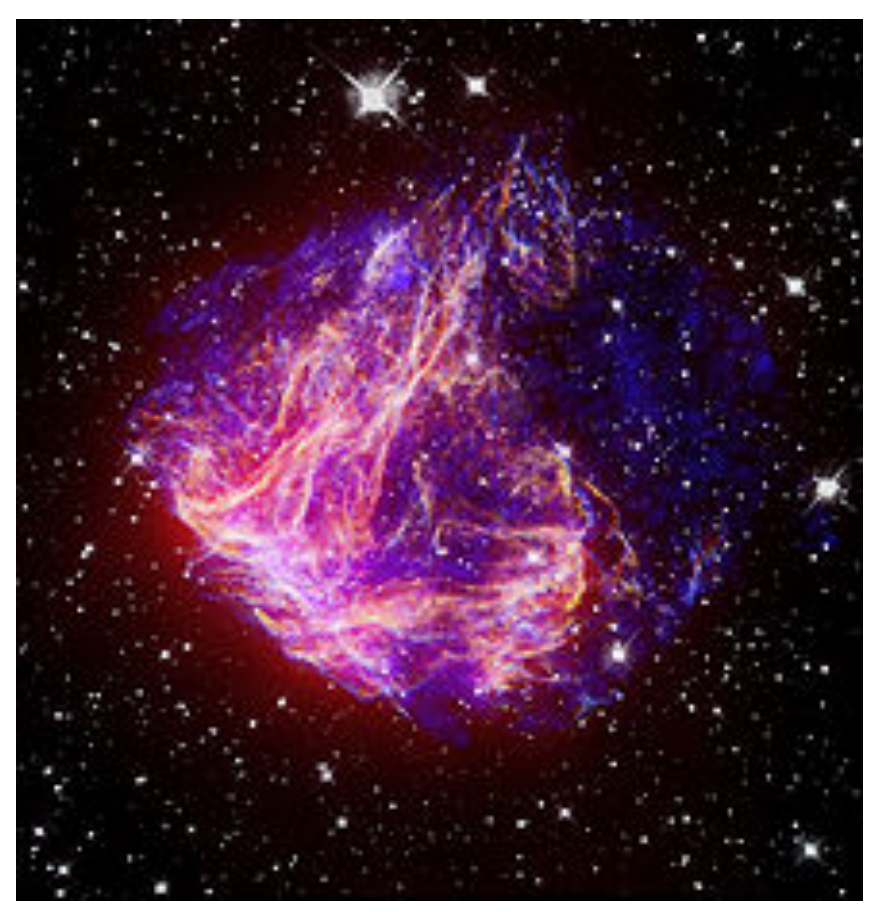
number initially $>1000$. Strong shock wave forms ahead of the ejecta, that heats the upstream Multiwavelength composite image of the supernova remnant N49 in the Large Magellanic Cloud. plasma up to temperatures well above millions of $\mathrm{K}$. 


\section{Astrophysical collisonless shocks}

- accelerate particles

- amplify magnetic fields (or create them)

- exchange energy between electrons and ions
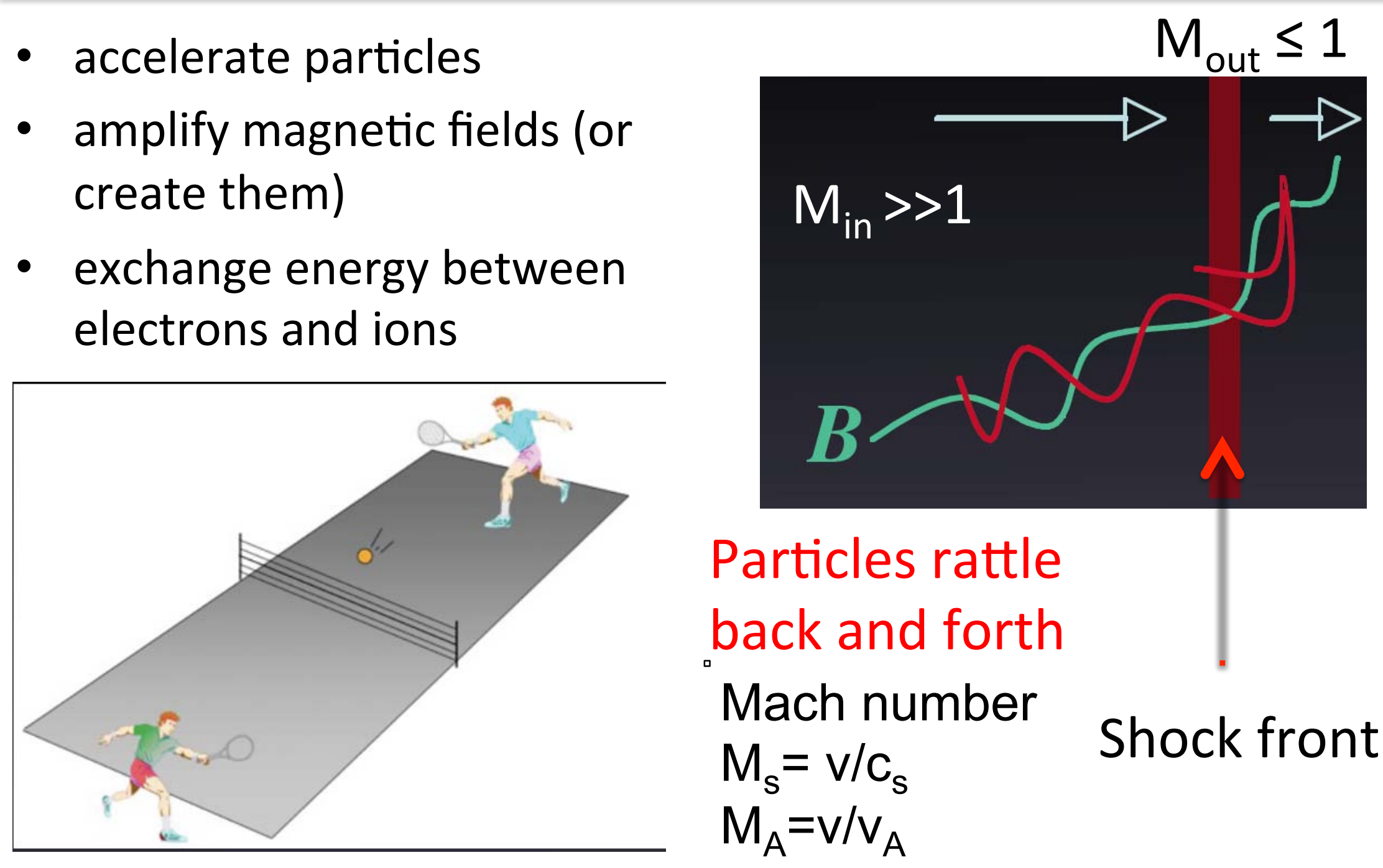

Particles rattle back and forth Mach number $\mathrm{M}_{\mathrm{s}}=\mathrm{v} / \mathrm{c}_{\mathrm{s}}$ $\mathrm{M}_{\mathrm{A}}=\mathrm{v} / \mathrm{v}_{\mathrm{A}}$ 


\section{Supernova Remnant (SNR)}

\section{Supernova Remnants}

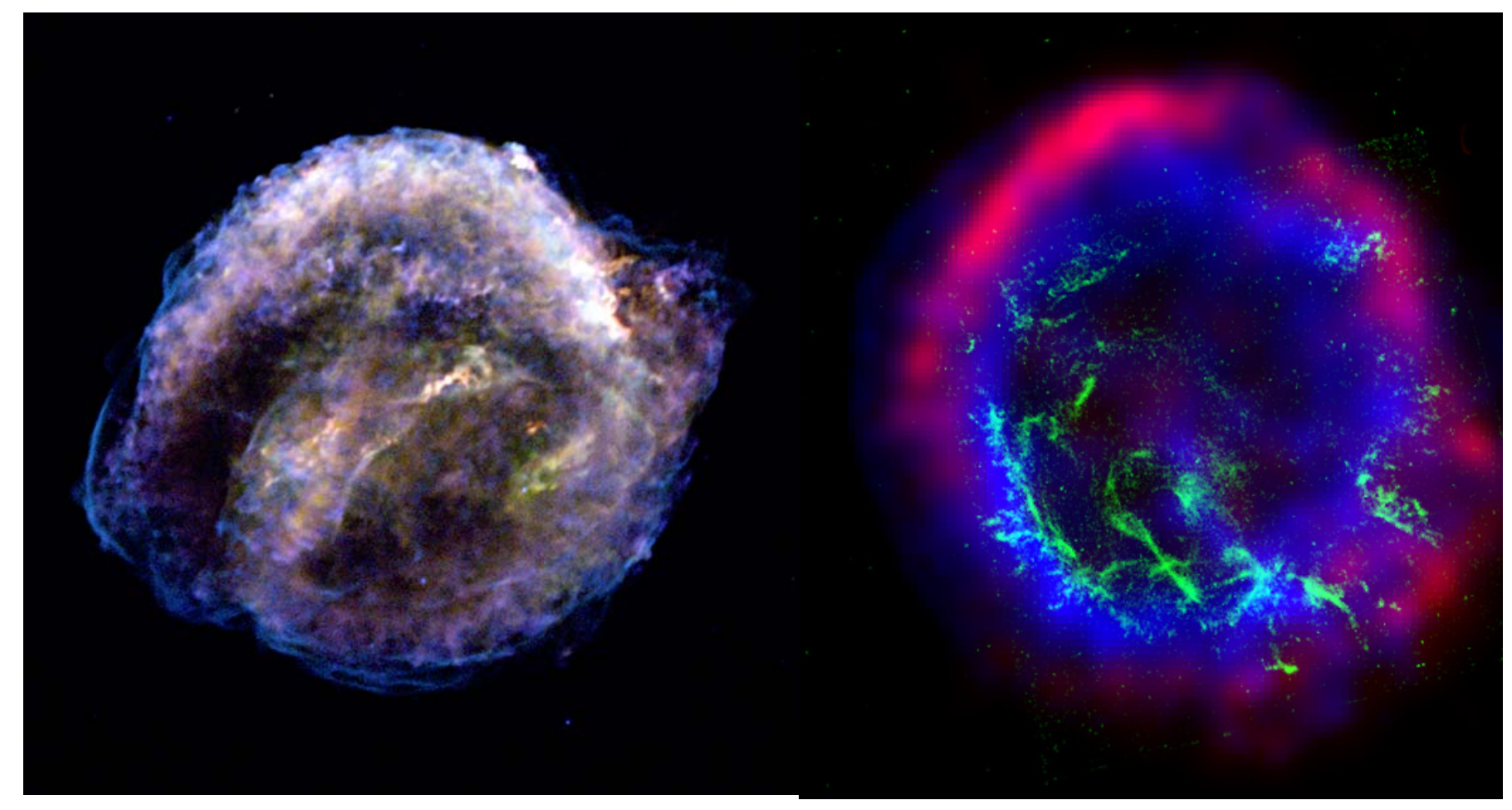

Kepler. Blue: highest energy X-ravs $\leftarrow \rightarrow$ shock e0102-723. blue: Chandra X-ray, million-K gas; red: radio, electrons 


\section{Rankine Hugoniot jump conditions}

- Adiabatic gas law

$-p V^{\gamma}=>p / \rho^{\gamma}=$ constant, $p=$ pressure, $\rho=$ mass density

- conservation of mass flow (in shock frame)

$-\rho_{0} v_{n 0}=\rho_{1} v_{n 1}, v=$ velocity

- conservation of momentum flux

$-\rho_{0} v_{n 0}^{2}+p_{0}=\rho_{1} v_{n 1}^{2}+p_{1}$

- Jump in energy flux

$-\varepsilon_{0}+v_{0}^{2} / 2+p_{0} / \rho_{0}=\varepsilon_{1}+v_{1}^{2} / 2+p_{1} / \rho_{1}$ 


\section{Supernova remnants show shock boundaries}

\section{Supernova Remnants Krauss-Varban, p7}

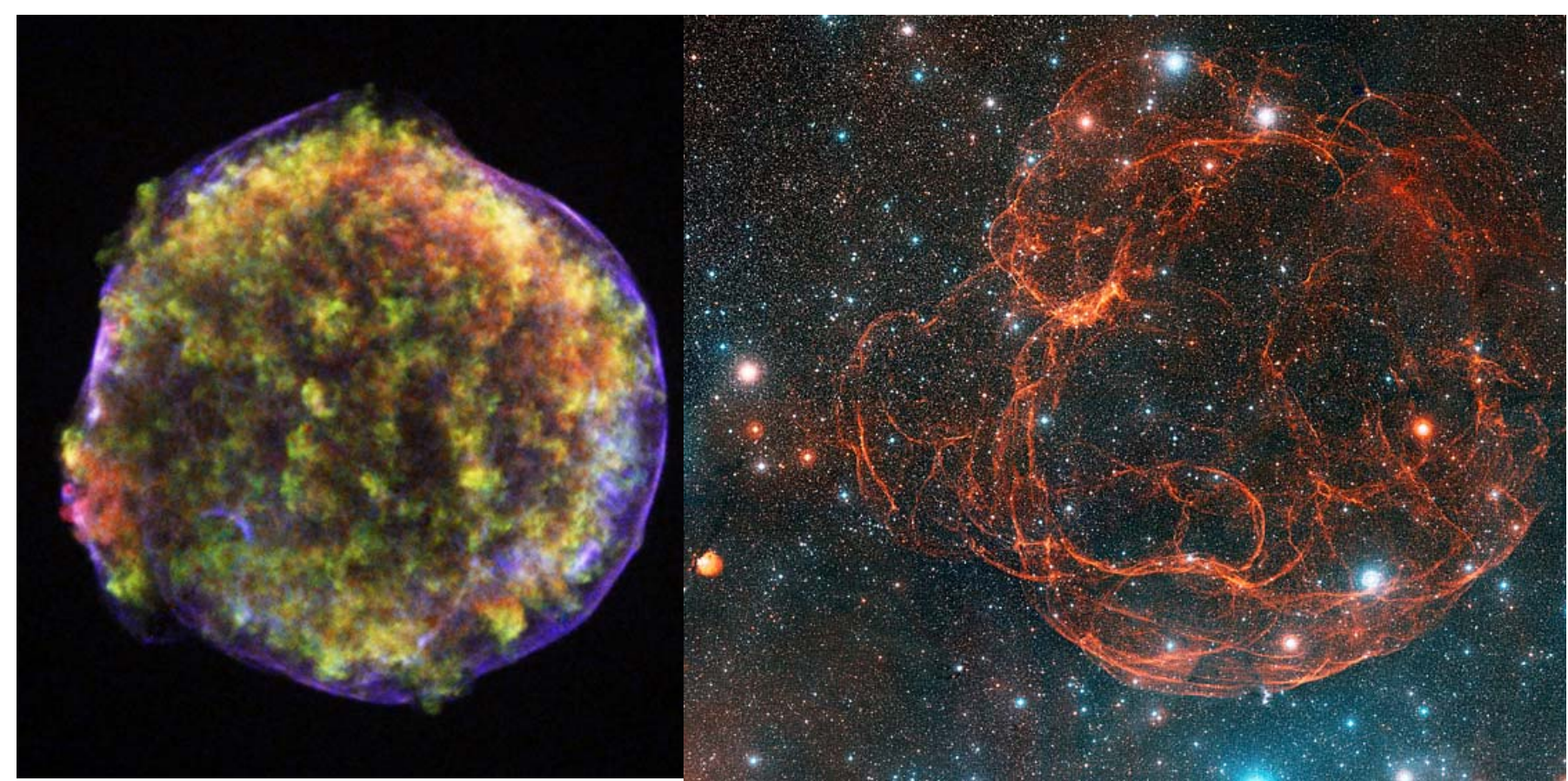

Tycho. Green, red: multimillion degree debris; blue: highenergy electrons. Nuclei
Simeis $147.3^{\circ}$ on the sky $\leftarrow \rightarrow$ 160 light-years wide @ 3,000 light-years distance 


\section{More SNR}

- Multiwavelength composite image of the remnant of Tycho's supernova, SN 1572.

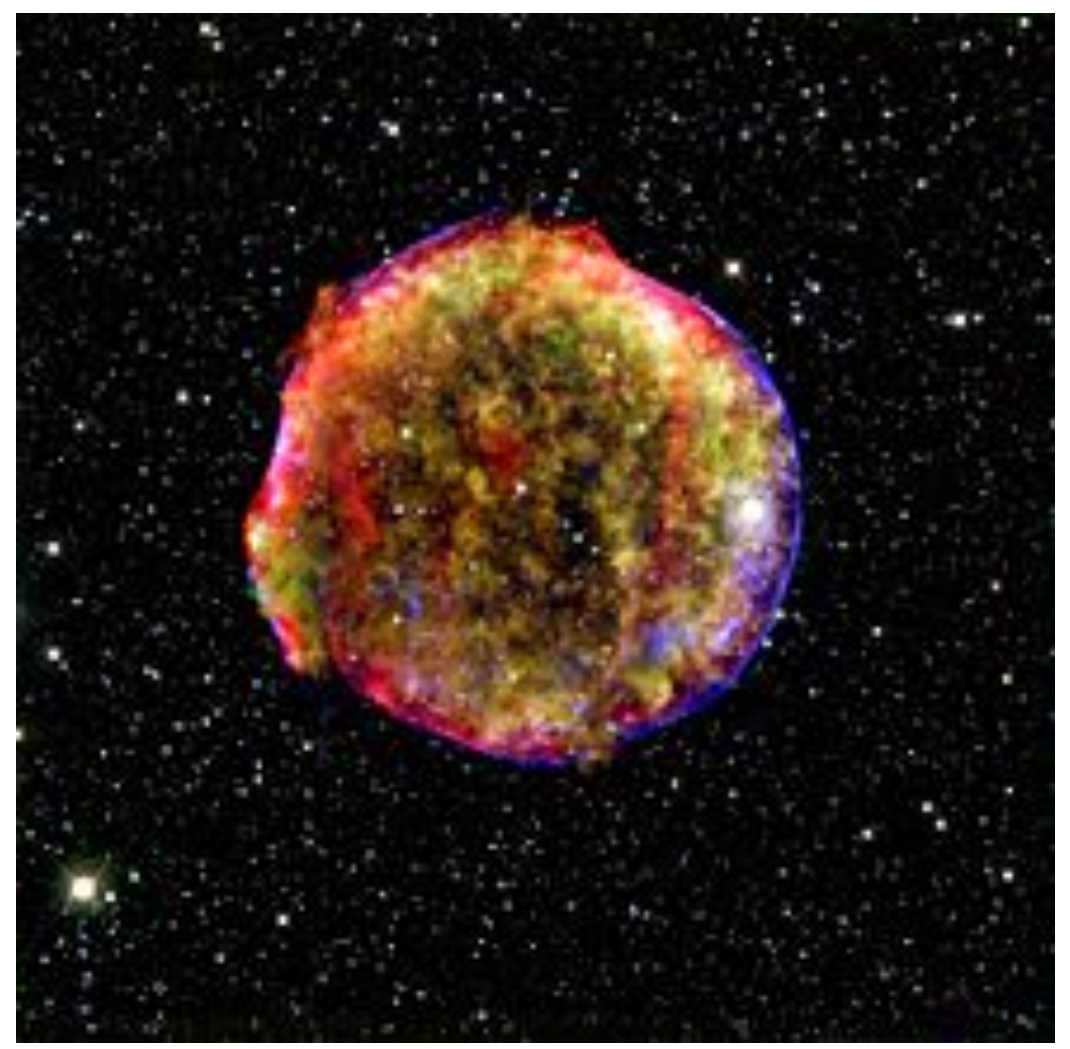




\section{Summary}

- Magnetic structure occurs from the smallest planetary to the largest cosmic scales.

- Introduce basic description some interesting features of non linear magnetohydrodynamics (MHD).

- dynamo creates magnetic field from flow

- reconnection annihilates magnetic field, accelerates particles.

- Shocks occur when flows move faster than the local velocity (sonic or Alfven speed) for the propagation of information.

- Reconnection, shocks, and ??? can accelerate particles. 


\section{Extra slides}




\section{Solar system confronts interstellar medium}

magnetic bubbles

- Earth

- solar system

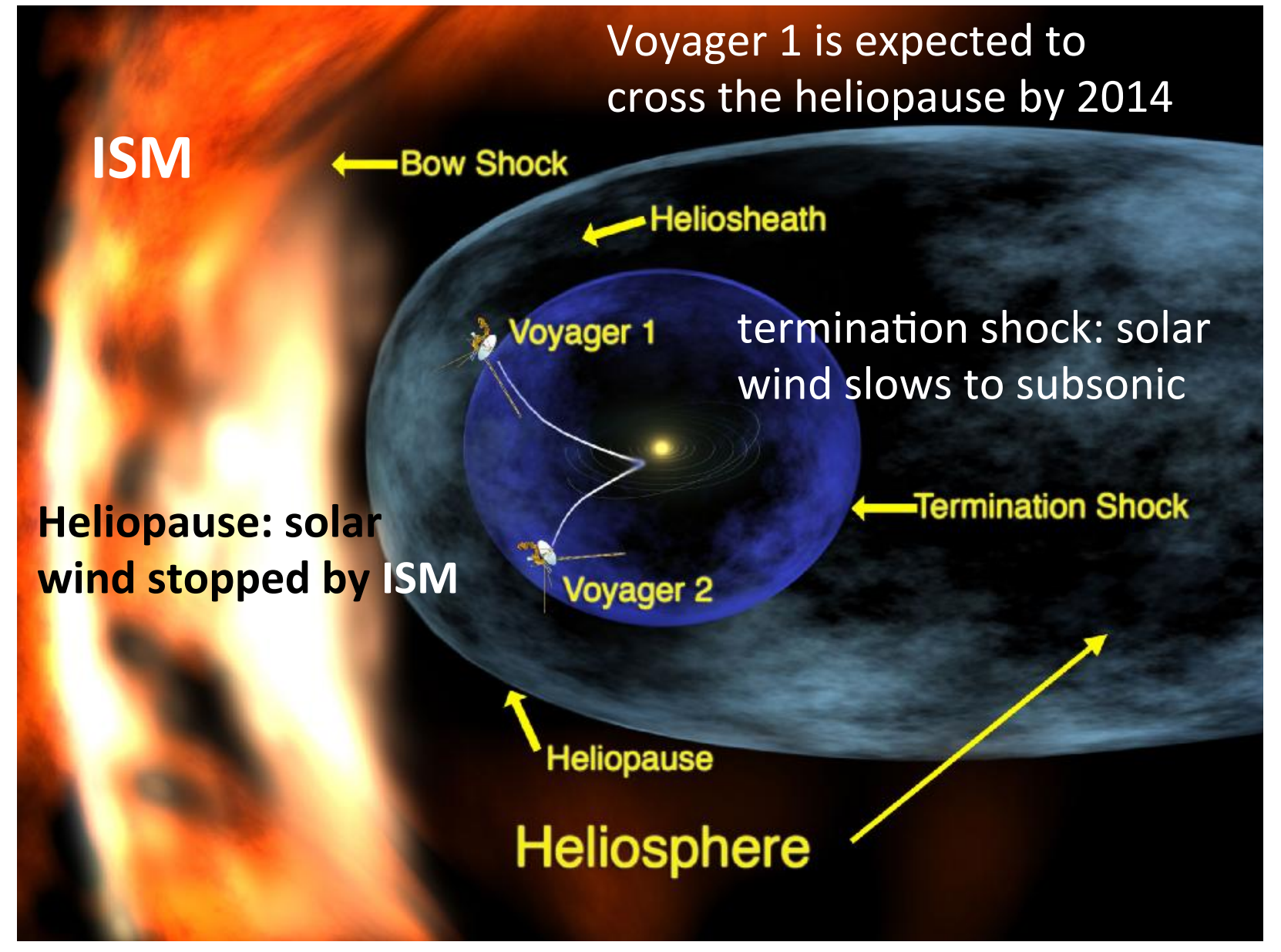

http://www.nasa.gov/images/content/462848main_helioshere_946-710.jpg 
Galactic jets - fluid flow and radiation powered by annihilation of magnetic fields (reconnection)?

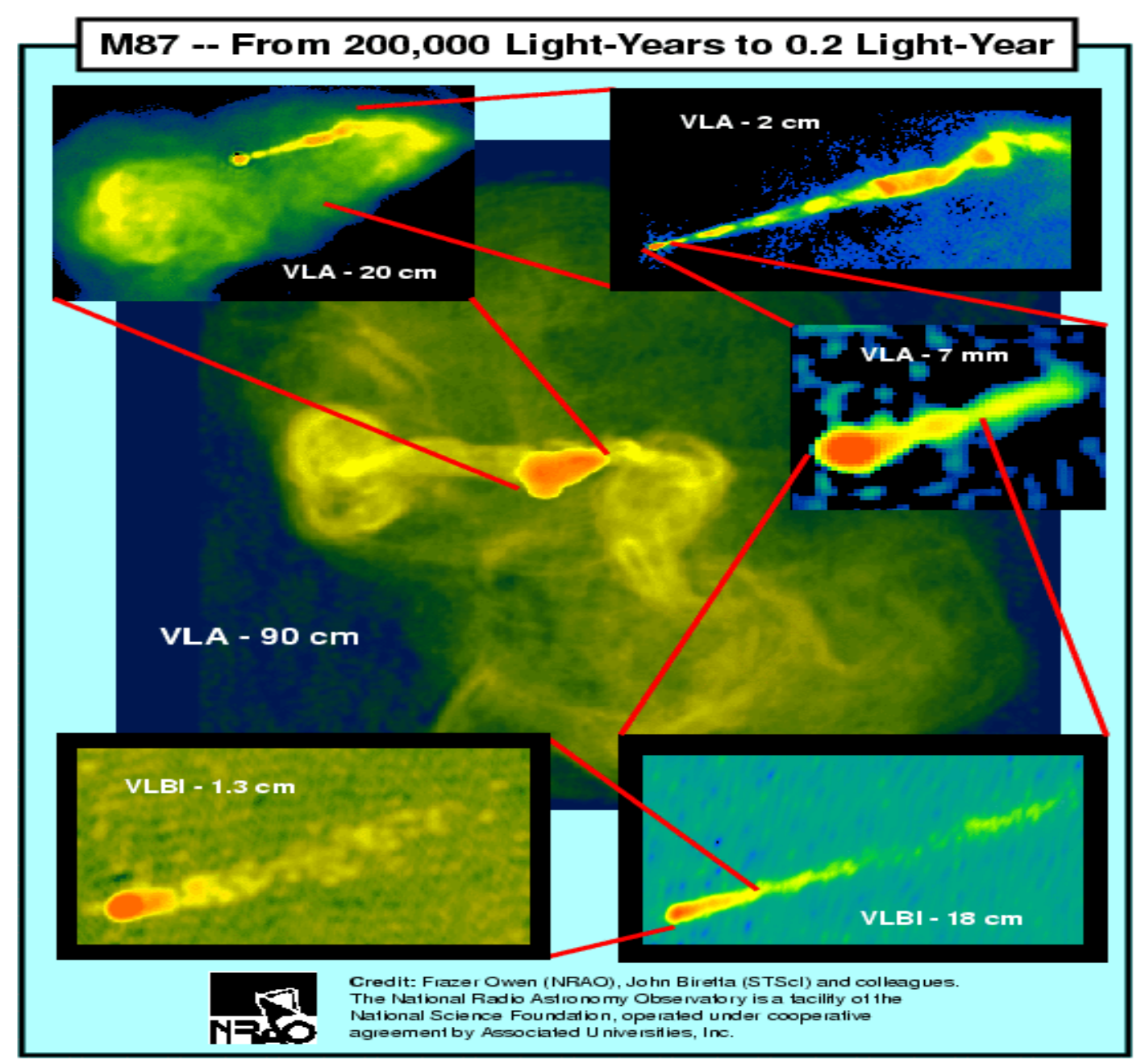




\section{Maxwell' s equations (SI units)}

describe electromagnetic fields

व

Faraday' s Law

Loop currents and magnetic field

Poisson' s eqn, charge density

Ampere' s law

Current conservation

Lorentz force equation

$$
\nabla \times E=-\frac{\partial B}{\partial t}
$$

$\nabla \cdot B=0$

$\nabla \cdot \varepsilon_{0} E=e\left(n_{i}-n_{e}\right)=\rho$

$\nabla \times B=\mu_{0} J+\frac{1}{c^{2}} \frac{\partial E}{\partial t}$

$\partial($ en $) / \partial \mathrm{t}+\nabla \bullet \mathrm{J}=0$

$F=q(E+v x B)$ 


\title{
Derivation of Plasma Fluid Equations Plasma Physics Summer School
}

\author{
Bruno Bauer \\ UNR Physics Department
}

Center for Nonlinear Studies, LANL

June 20, 2012
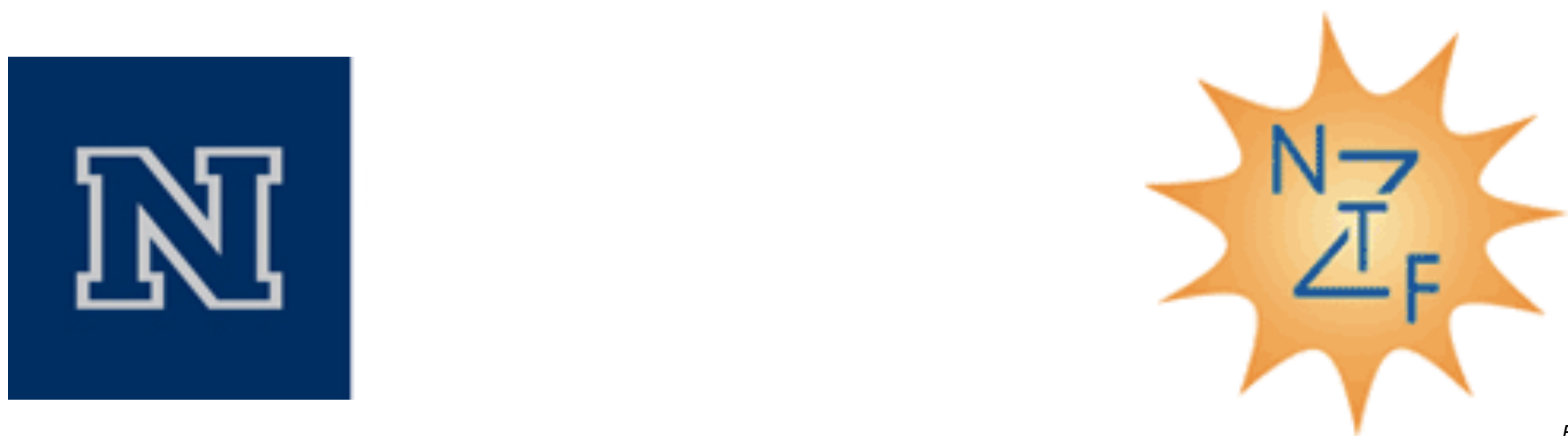


\section{Abstract}

Plasma physics is of great importance for science and technology. All plasma follows a common set of principles, whether it is the tenuous plasma of interstellar space or the ultradense plasma created in inertial confinement fusion experiments; or whether it is the cool, chemical plasma used in the processing of semiconductors or the hot, thermonuclear plasma of stars and fusion devices. This second lecture of the Plasma Physics Summer School continues a broad outline of plasma physics. Starting with particle motion, we build distribution functions and derive kinetic equations. Taking velocity moments of the Boltzmann kinetic equation we reduce the dimensionality of the plasma description but increase the number of partial differential equations. Through suitable approximations, we obtain and close fluid equations, including those of magnetohydrodynamics. At every stage in this cascade of derivations, we should pause to solve our equations and admire a new perspective on the wondrous world of plasma equilibria, waves, and instabilities. 


\section{Derivation of Plasma Fluid Eqns}

1. The challenge of plasma physics

2. Kinetic equations

- Klimontovich, Boltzmann, Vlasov

- Distribution functions

3. Particle motion

4. Velocity moments of Boltzmann equation + approximations

=> fluid equations \& magnetohydrodynamics

5. Plasma equilibria, waves, instabilities, self-organization 


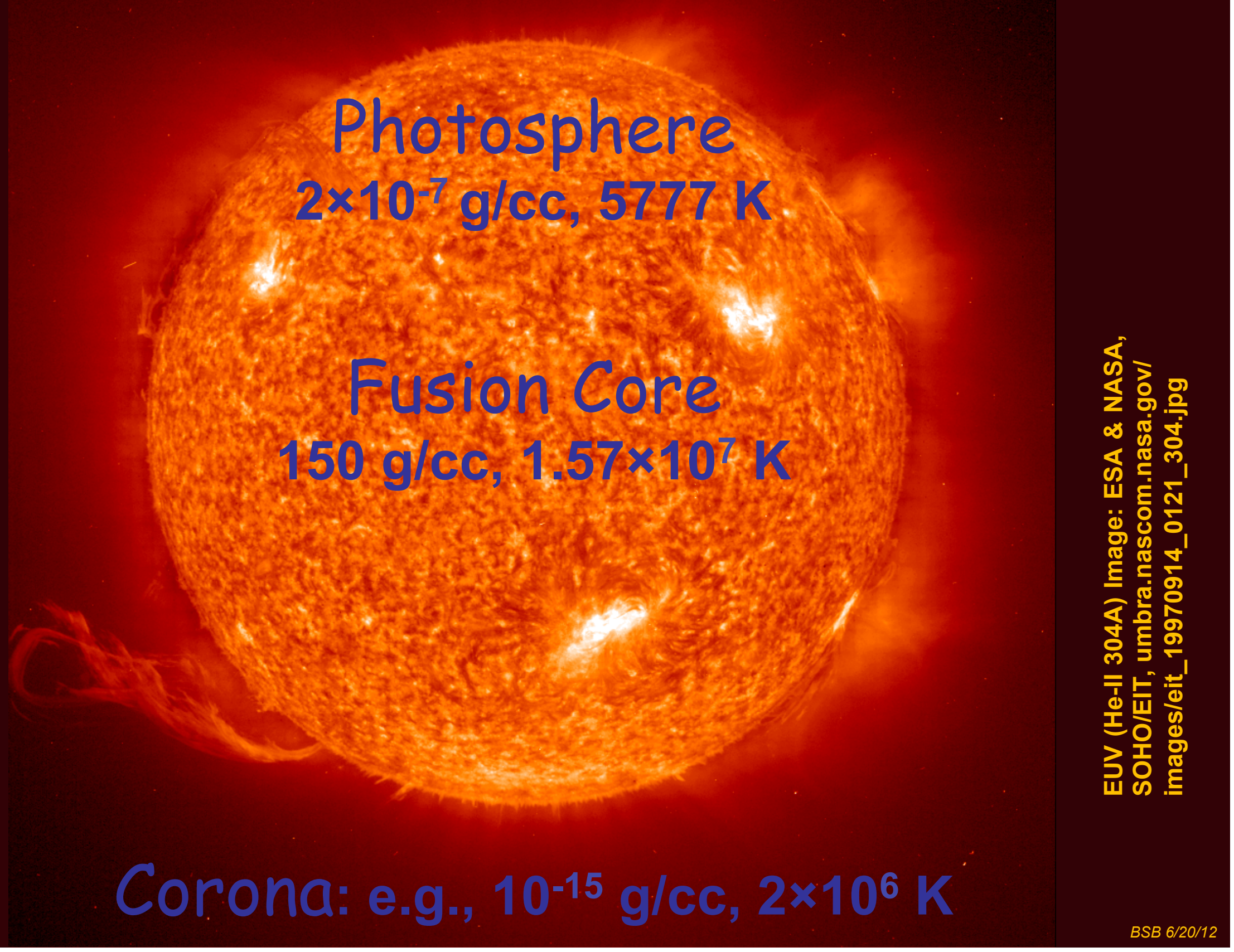




\section{Solar wind drives Earth magnetosphere}
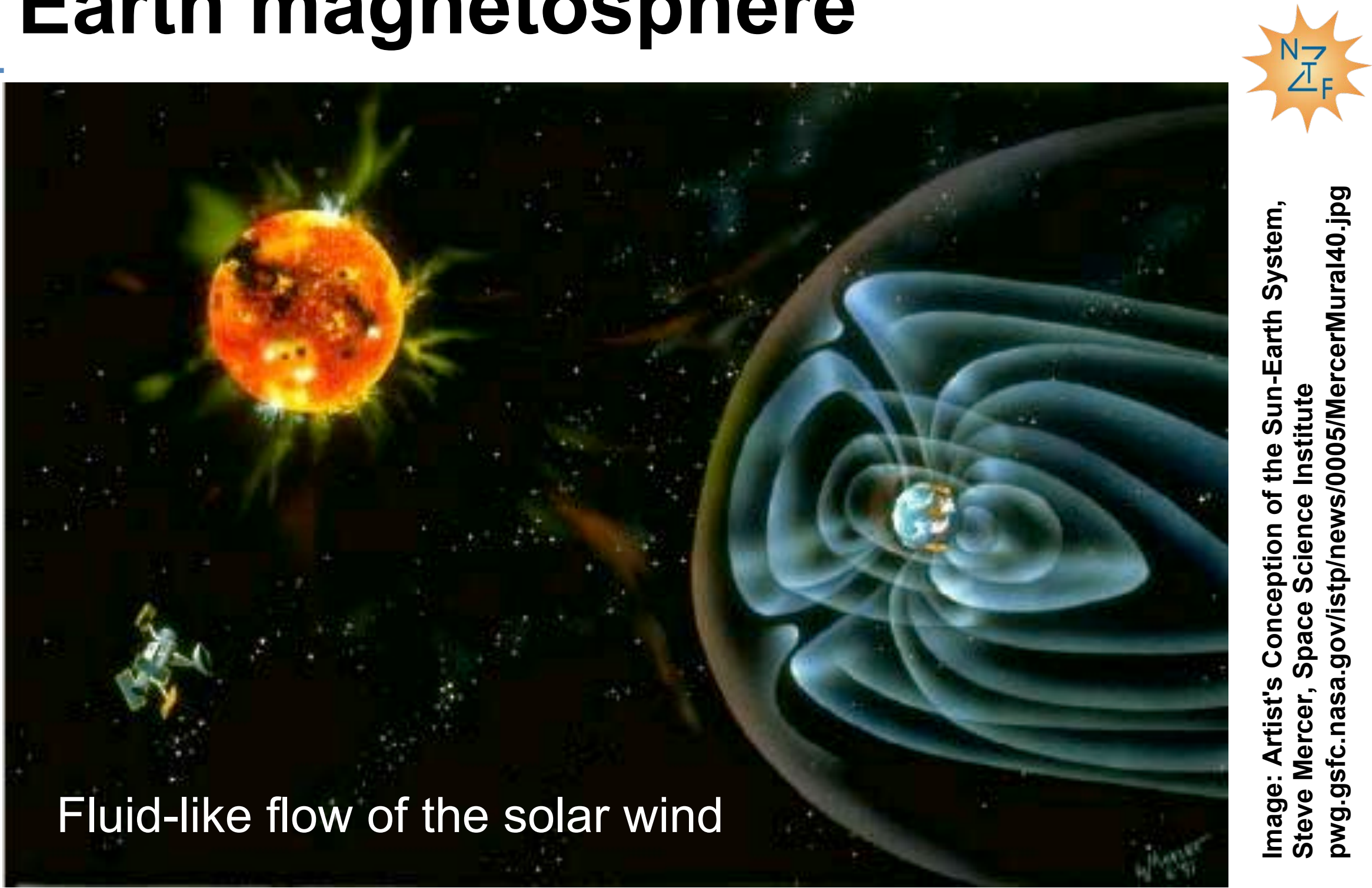


\section{The challenge of plasma physics}

$>$ Explain macrostructure from microphysics

$>$ Understand \& control large collections of unbound charged particles \& photons

Classical plasma physics simplifying assumption:

* Assume plasma parameters such that particles can be described with classical physics

$$
\begin{aligned}
& \text { For } i=1,2,3, \mathrm{~K}, N: \quad\left\{q_{i}, m_{i}, r_{i}, v_{i}, a_{i}\right\}
\end{aligned}
$$

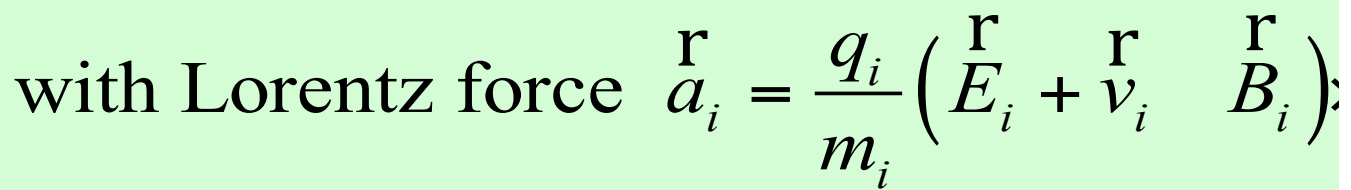

where spiky fields are determined by the particles via Maxwell's equations

* This is a complete, but intractable, set of equations 


$$
\begin{aligned}
& \text { Maxwell's Equations } \\
& \oint \vec{E} \cdot d \vec{A}=\frac{Q}{\varepsilon_{o}} \\
& \text { Gauss' } \\
& \text { Laws } \\
& \oint \vec{B} \cdot d \vec{A}=0 \\
& \oint \vec{E} \cdot d \vec{l}=-\frac{d \Phi_{B}}{d t} \quad \begin{array}{l}
\text { Faraday's } \\
\text { Law }
\end{array} \\
& \text { Relates } \boldsymbol{E} \text { to charges. } \\
& \text { (Coulomb's law) } \\
& \boldsymbol{B} \text { is continuous. } \\
& \text { No magnetic monopoles. } \\
& \oint \vec{B} \cdot d \vec{l}=\mu_{o} I+\mu_{o} \varepsilon_{o} \frac{d \Phi_{E}}{d t}{ }_{\text {Law }}^{\begin{array}{l}
\text { Ampère- } \\
\text { Laxwell }
\end{array}} \begin{array}{l}
\boldsymbol{I} \text { or Changing } \boldsymbol{E} \\
\text { produces } \boldsymbol{B}
\end{array}
\end{aligned}
$$

These equations describe all electric \& magnetic phenomena (in the absence of dielectric or magnetic materials). 


\section{Maxwell's equations for spiky fields}

$$
\begin{aligned}
& \stackrel{\mathrm{r}}{\nabla \mathrm{g} E} \underset{\mathrm{r}}{\mathrm{r}}=\frac{\rho}{\varepsilon_{0}} \text { where } \rho(\stackrel{\mathrm{r}}{r}, t)=\sum_{i=1}^{N} q_{i} \delta\left(\stackrel{\mathrm{r}}{r}-\underset{r_{\mathrm{i}}}{\mathrm{r}}(t)\right) \quad \rho_{\text {ett }}(\stackrel{\mathrm{r}}{r}, t) \\
& \stackrel{\mathrm{r}}{\nabla} \times \stackrel{\mathrm{r}}{E}=-\frac{\partial \stackrel{\mathrm{B}}{\partial t}}{\partial t}
\end{aligned}
$$

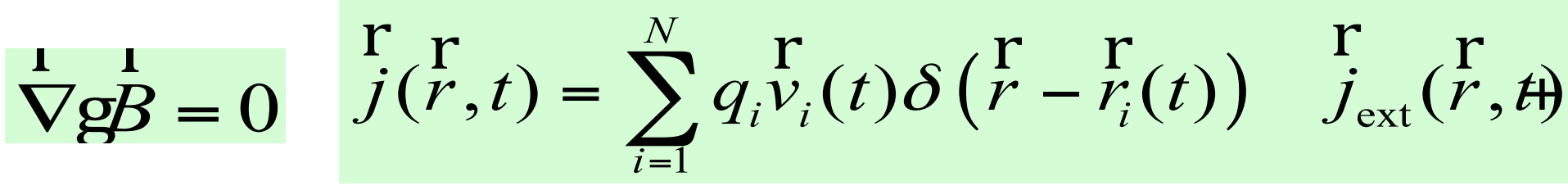

$$
\begin{aligned}
& \stackrel{\mathrm{r}}{\nabla} \times \stackrel{\mathrm{r}}{B}=\mu_{0} \stackrel{\mathrm{r}}{j}+\varepsilon_{0} \frac{\partial \stackrel{t}{E}}{\partial t}
\end{aligned}
$$




\section{Delta functions: $\int \delta(x) d x=1$, $\delta(0)=\infty, \delta(x)=0$ for $x \neq 0$}
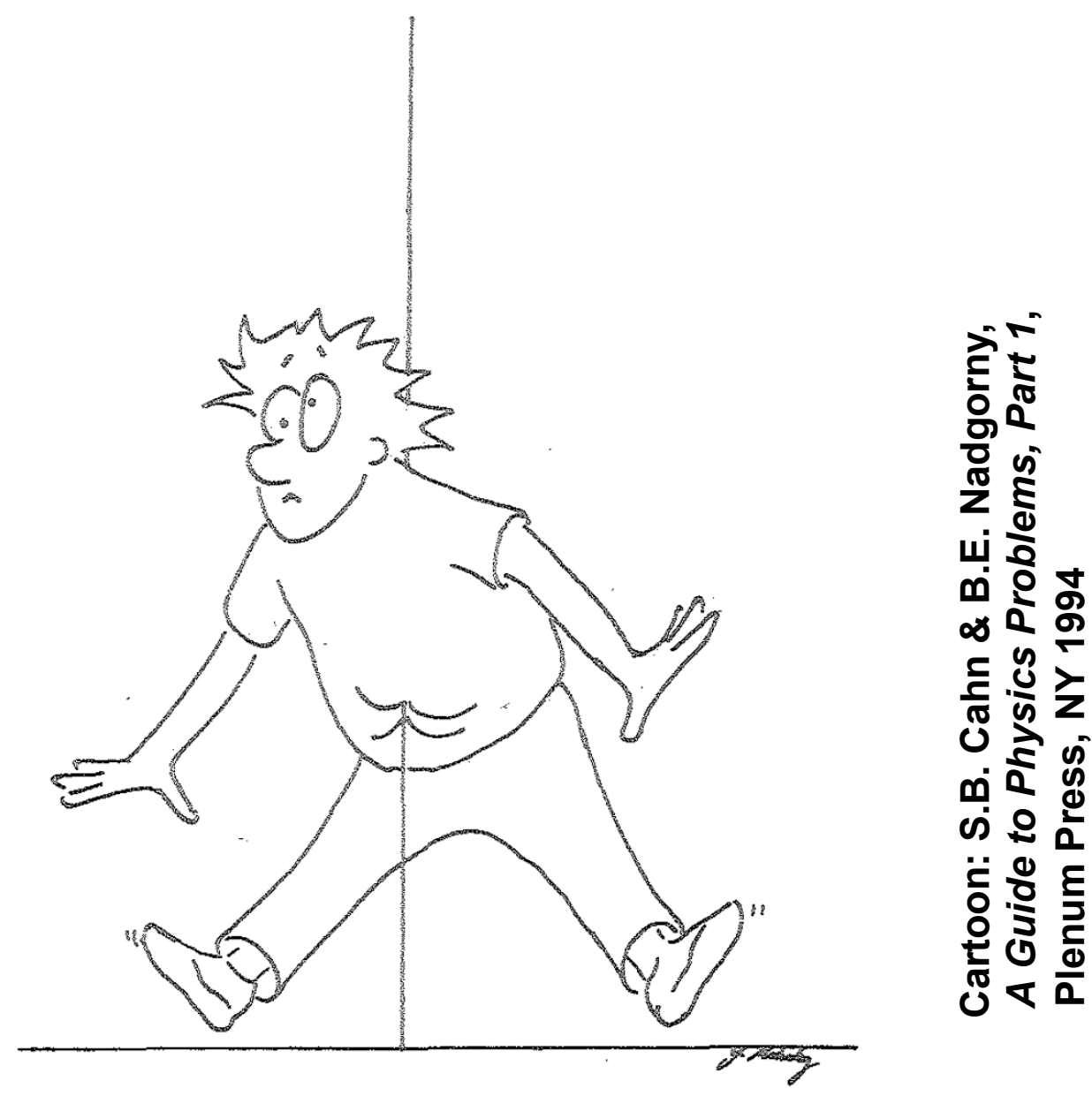

Figure P.1

Hapless Physicist Impaled on his own Delta Function (Demonstrating the Perils of Insufficient Theoretical Rigor) 


\section{Derivation of Plasma Fluid Eqns}

1. The challenge of plasma physics

2. Kinetic equations

- Klimontovich, Boltzmann, Vlasov

- Distribution functions

3. Particle motion

4. Velocity moments of Boltzmann equation + approximations

=> fluid equations \& magnetohydrodynamics

5. Plasma equilibria, waves, instabilities, self-organization 


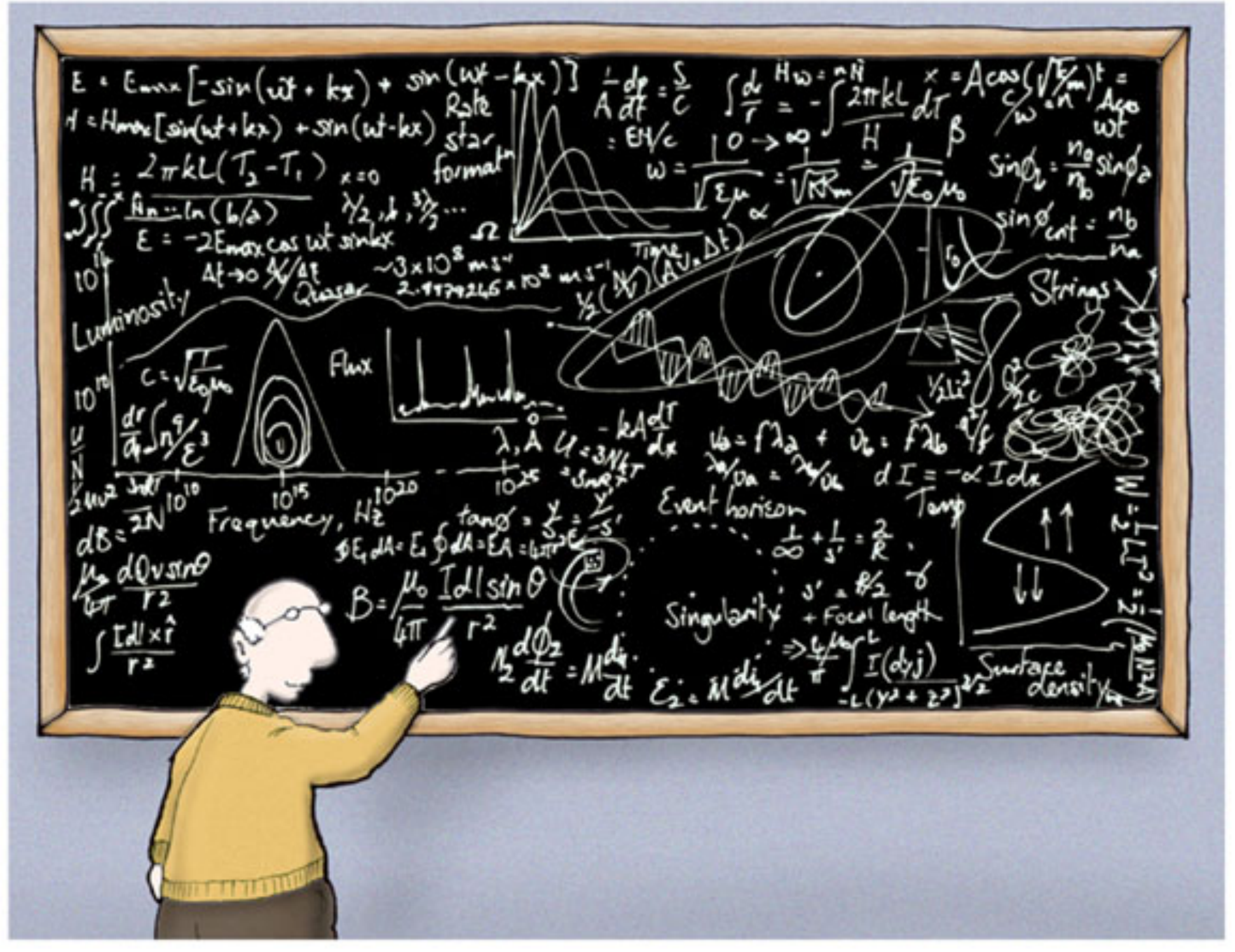

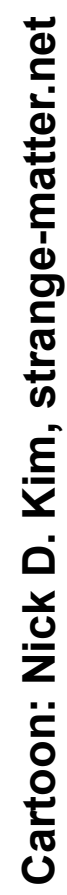

Astrophysics made simple 


\section{Take a statistical approach to tracking the large number of plasma particles}

For each species of particle, $\alpha$, define the spiky microscopic phase-space distribution function of Klimontovich

$$
f_{\mathrm{K} \alpha}\left(\stackrel{\mathrm{r}}{\stackrel{\mathrm{r}}{v}, t)}=\sum_{i=1}^{N_{\alpha}} \delta\left(\stackrel{\mathrm{r}}{r}-r_{i}^{\mathrm{r}}(t)\right) \delta\left(\stackrel{\mathrm{r}}{v}-\underset{v_{i}}{\mathrm{r}}(t)\right)\right.
$$

Here $N_{\alpha}=$ \# of particles of species $\alpha$ 


\section{Differentiating Klimontovich distribution $=>$ Klimontovich equation}

$$
\begin{aligned}
& f_{\mathrm{K} \alpha}(\stackrel{\mathrm{r}}{r}, \stackrel{\mathrm{r}}{v}, t)=\sum_{i=1}^{N_{\alpha}} \delta\left(\stackrel{\mathrm{r}}{r}-\underset{r_{i}}{\mathrm{r}}(t)\right) \delta\left(\stackrel{\mathrm{r}}{v}-\underset{v_{i}}{\mathrm{r}}(t)\right) \\
& \frac{\partial f_{\mathrm{K} \alpha}}{\partial t}=-\frac{d r_{i}}{d t} \mathrm{~g}{ }^{\mathrm{r}} f_{\mathrm{K} \alpha}-\frac{d v_{i}}{d t} \mathrm{~g} \nabla_{v} f_{\mathrm{K} \alpha}
\end{aligned}
$$

Let the delta functions do the localizing to particles:

$$
\frac{\partial f_{\mathrm{K} \alpha}}{\partial t}=-\stackrel{\mathrm{r}}{v} \mathrm{~g} \nabla f_{\mathrm{K} \alpha}^{\mathrm{r}}-\stackrel{\mathrm{r}}{a} \mathrm{~g} \nabla_{v}^{\mathrm{r}} f_{\mathrm{K} \alpha}
$$

Inserting Lorentz force $=>$ Klimontovich equation:

$$
\frac{\partial f_{\mathrm{K} \alpha}}{\partial t}+\stackrel{\mathrm{r}}{v} \mathrm{~g} \stackrel{\mathrm{r}}{\mathrm{\nabla}} f_{\mathrm{K} \alpha}+\frac{q}{m}(\stackrel{\mathrm{r}}{E}+\stackrel{\mathrm{r}}{v} \times \stackrel{\mathrm{r}}{B}) \stackrel{\mathrm{r}}{\mathrm{g}}{ }_{v} f_{\overline{\mathrm{K} \alpha}} \quad 0
$$




\section{The Klimontovich equation conserves particle number and spikiness}

$$
\begin{aligned}
& f_{\mathrm{K} \alpha}(\stackrel{\mathrm{r}}{r}, \stackrel{\mathrm{r}}{v}, t)=\sum_{i=1}^{N_{\alpha}} \delta\left(\stackrel{\mathrm{r}}{r}-\underset{r_{i}}{\mathrm{r}}(t)\right) \delta\left(\stackrel{\mathrm{r}}{v}-v_{i}^{\mathrm{r}}(t)\right)
\end{aligned}
$$

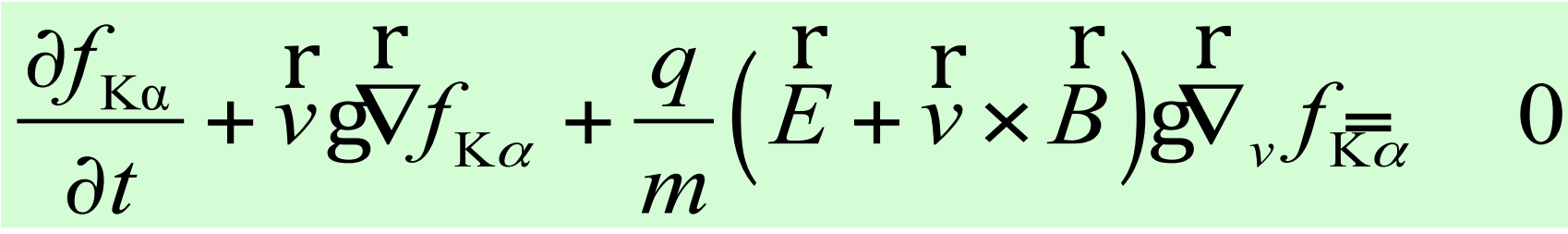

Recognizing convective derivative: $\quad \frac{\mathrm{d} f_{\mathrm{K} \alpha}}{\mathrm{d} t}=0$ 


\section{Ensemble average to obtain smooth functions and the Boltzmann equation}

$$
\begin{aligned}
& f_{\mathrm{K} \alpha}(\stackrel{\mathrm{r}}{r}, \stackrel{\mathrm{r}}{v}, t)=\sum_{i=1}^{N_{\alpha}} \delta(\stackrel{\mathrm{r}}{r}-\underset{r}{r}(t)) \delta\left(\stackrel{\mathrm{r}}{v}-\underset{v_{i}}{\mathrm{r}}(t)\right) \\
& \frac{\partial f_{\mathrm{K} \alpha}}{\partial t}+\stackrel{\mathrm{r}}{v} \stackrel{\mathrm{r}}{\mathrm{g} \nabla} f_{\mathrm{K} \alpha}+\frac{q}{m}(\stackrel{\mathrm{r}}{E}+\stackrel{\mathrm{r}}{v} \times \stackrel{\mathrm{r}}{B}) \stackrel{\mathrm{r}}{\mathrm{g}}{ }_{v} f_{\overline{\mathrm{K} \alpha}} \quad 0 \\
& f_{\alpha} \equiv<f_{\mathrm{K} \alpha}>\text {, } \\
& \underset{\mathrm{r}}{\delta f_{\alpha}} \equiv f_{\mathrm{r} \alpha}-f_{\alpha} \text {, } \\
& \stackrel{\mathrm{r}}{E} \equiv<\stackrel{\mathrm{r}}{E}_{K}>\text {, etc. } \quad \Rightarrow \text { the Boltzmann equation: } \\
& \frac{\mathrm{d} f_{\alpha}}{\mathrm{d} t}=\frac{\partial f_{\alpha}}{\partial t}+\stackrel{\mathrm{r}}{v} \stackrel{\mathrm{r}}{\mathrm{g}} f_{\alpha}+\frac{q}{m}(\stackrel{\mathrm{r}}{E}+\stackrel{\mathrm{r}}{v} \times \stackrel{\mathrm{r}}{\mathrm{B}}) \stackrel{\mathrm{r}}{\mathrm{g} \nabla_{v}} f_{\alpha}=\left(\frac{\partial f_{\alpha}}{\partial t}\right)_{\mathrm{c}}
\end{aligned}
$$




\section{A distribution function $f\left(x, y, z, v_{x}, v_{y}, v_{z}\right)$ is a density in phase space}

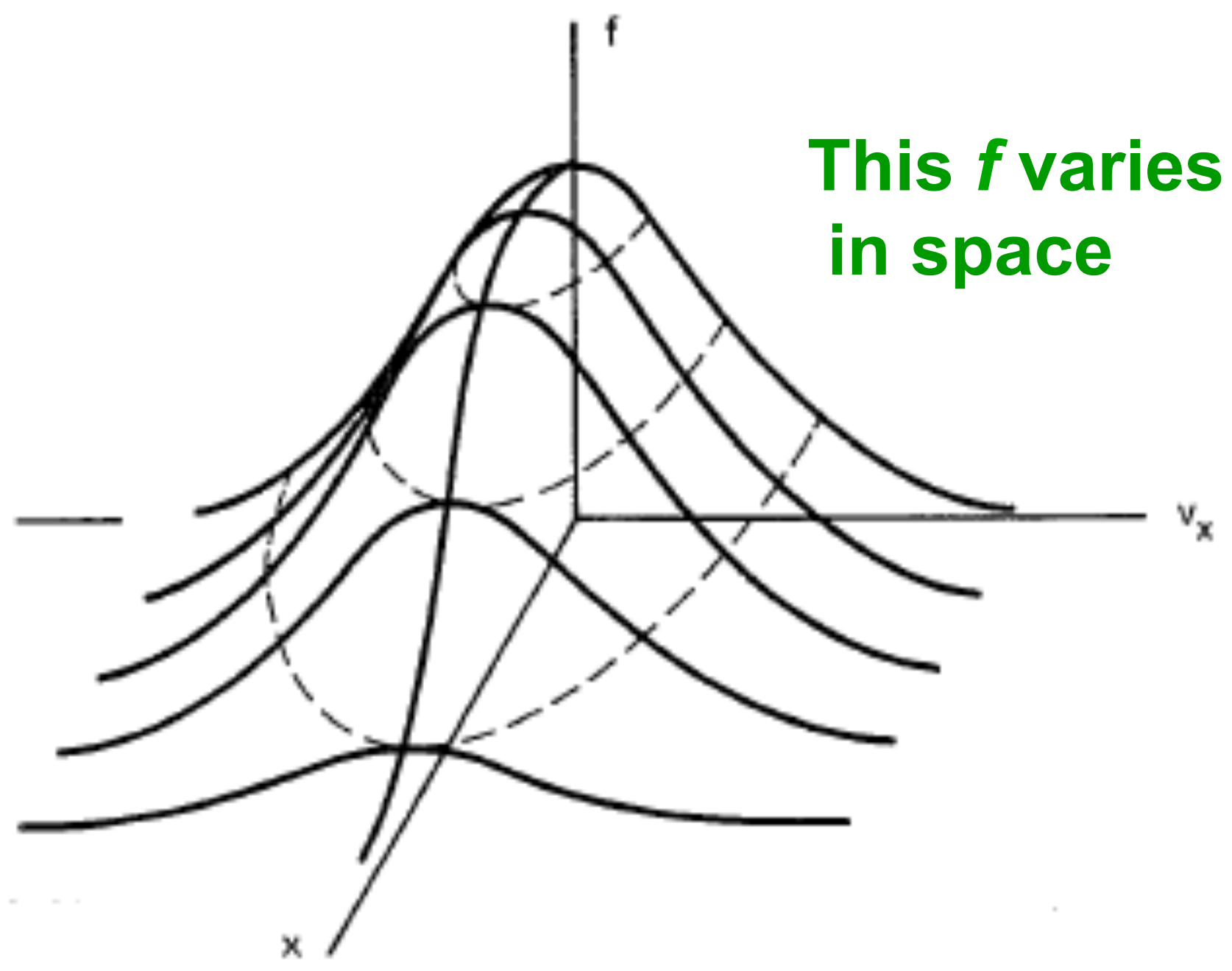

Figure: F.F. Chen, Introduction to Plasma Physics and Controlled Fusion, Vol. 1, $2^{\text {nd }}$ ed., Plenum Press, NY, 1984. 


\section{Collisions drive the plasma toward maximum entropy}

$$
\begin{aligned}
& f_{\alpha}(\stackrel{\mathrm{r}}{r}, \underset{v}{\mathrm{r}})=\left.n_{\alpha}\right|_{\phi=0} \exp \left[\frac{-q_{\alpha} \phi(\stackrel{\mathrm{r}}{r})}{k_{\beta} T_{\alpha}}\right] f_{\mathrm{M}}(\stackrel{\mathrm{r}}{v}) \\
& f_{\mathrm{M}}(\stackrel{\mathrm{r}}{v})=\frac{1}{a^{3} \pi^{3 / 2}} e^{-v^{2} / a^{2}}, \quad a \equiv \sqrt{\frac{2 k_{\beta} T_{\alpha}}{m_{\alpha}}}
\end{aligned}
$$

Maxwellian distribution function 


\section{Plasma distribution functions are often non-Maxwellian}
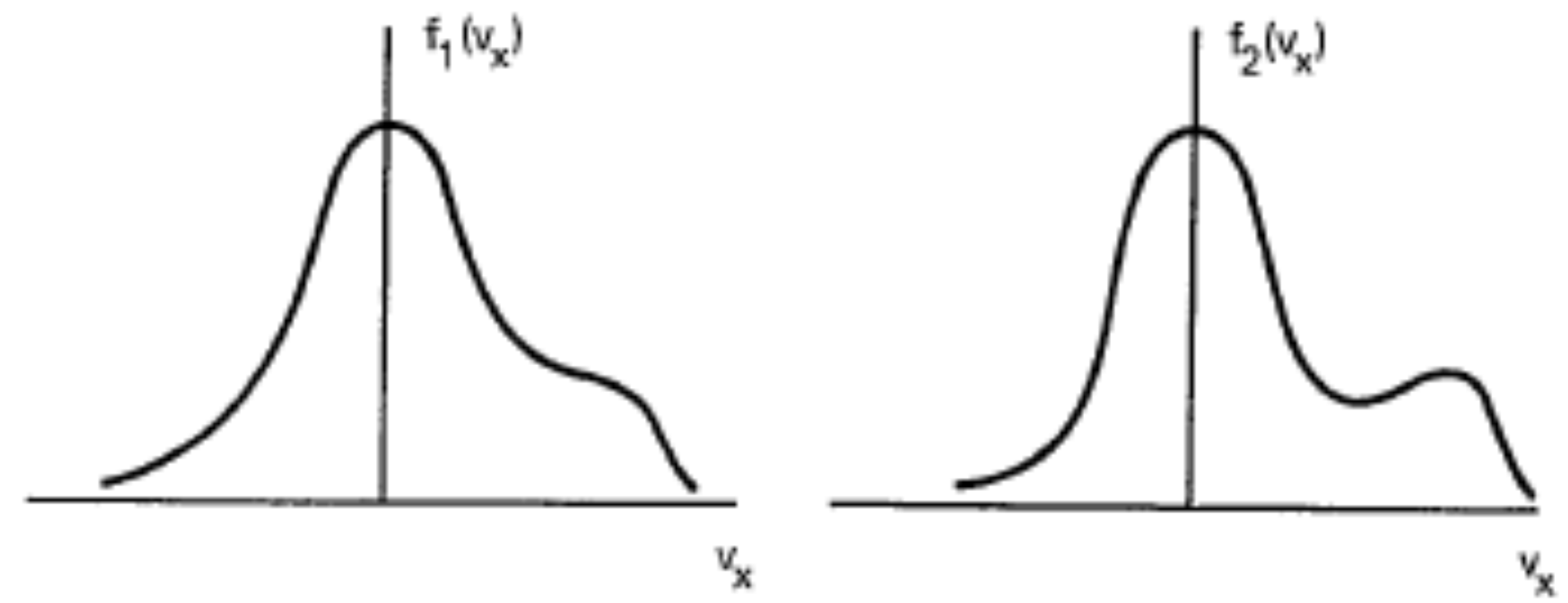

Figure: F.F. Chen, Introduction to Plasma Physics and Controlled Fusion, Vol. 1, $2^{\text {nd }}$ ed., Plenum Press, NY, 1984. 


\section{Plasma distribution functions are often anisotropic}

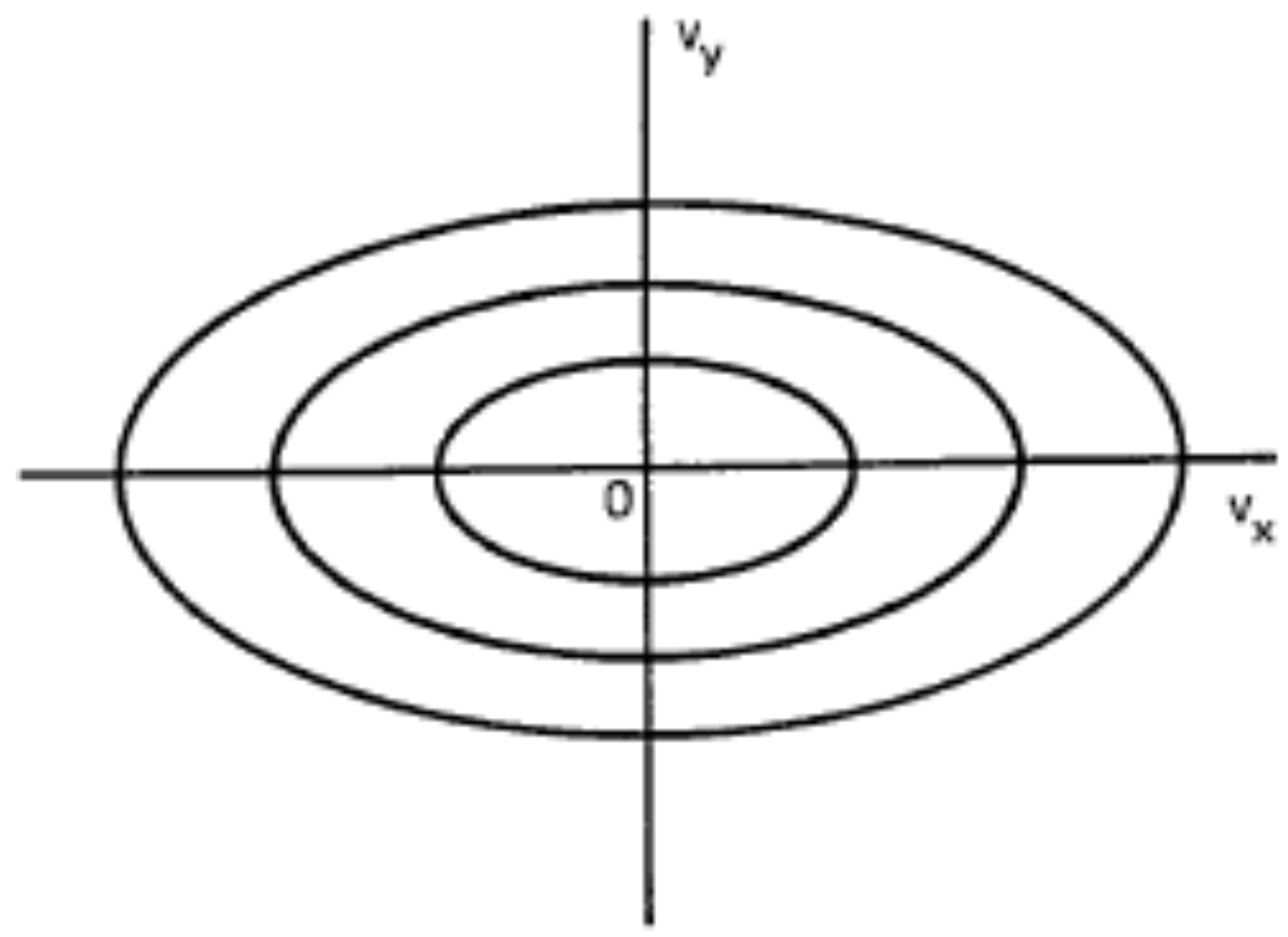

Figure: F.F. Chen, Introduction to Plasma Physics and

Controlled Fusion, Vol. 1, $2^{\text {nd }}$ ed., Plenum Press, NY, 1984. 


\section{Phase-space evolution can be complex and beautiful}

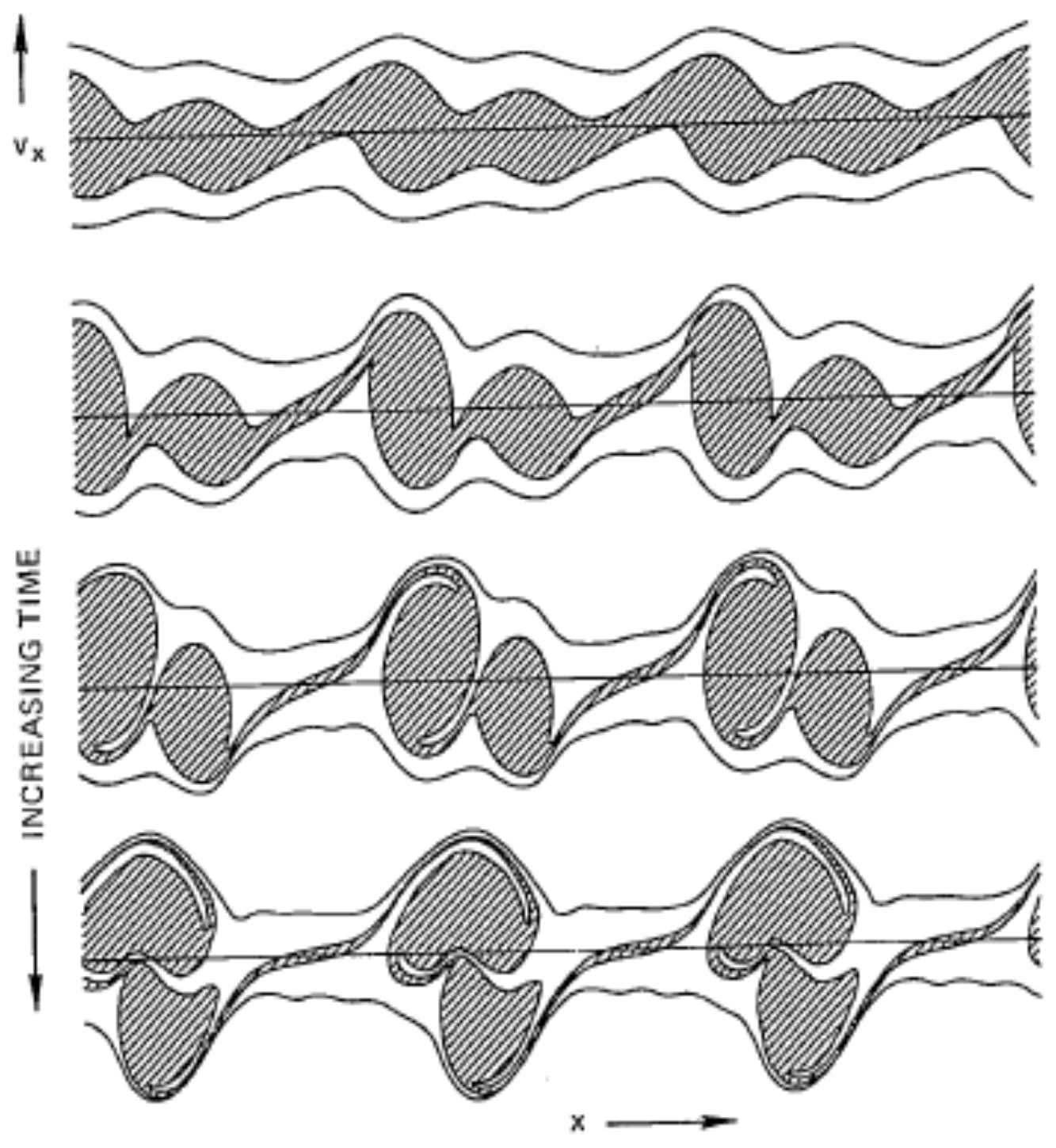

Figure: Electron 2-stream instability, H.L. Berk, C.E. Nielson, and K.V. Roberts, Phys. Fluids 13, 986 (1970). In F.F. Chen, Introduction to Plasma Physics and Controlled Fusion, Vol. 1, $2^{\text {nd }}$ ed., Plenum Press, NY, 1984. 


\section{To better understand Boltzmann equation, follow particles in phase space \& calculate collisions}

$$
\frac{\mathrm{d} f_{\alpha}}{\mathrm{d} t}=\frac{\partial f_{\alpha}}{\partial t}+\underset{v}{\mathrm{r}} \mathrm{g} \stackrel{\mathrm{r}}{\nabla} f_{\alpha}+\frac{q}{m}(\stackrel{\mathrm{r}}{E}+\underset{v}{\mathrm{r}} \times \stackrel{\mathrm{r}}{B}) \underset{\mathrm{g}}{\mathrm{r}} f_{\nu} f_{\alpha}=\left(\frac{\partial f_{\alpha}}{\partial t}\right)_{\mathrm{c}}
$$

One Boltzmann equation per species of particle

$>$ Describes the evolution of the distribution of particles in phase-space

How do particles flow in phase space?

$>$ What is the effect of the collision term (on rhs)? How would $f$ evolve without collisions?

\section{Neglect collisions $=>$ Vlasov equation}

$$
\frac{\mathrm{d} f_{\alpha}}{\mathrm{d} t}=\frac{\partial f_{\alpha}}{\partial t}+\stackrel{\mathrm{r}}{v} \underset{\mathrm{g}}{\mathrm{r}} f_{\alpha}+\frac{q}{m}(\stackrel{\mathrm{r}}{E}+\stackrel{\mathrm{r}}{v} \times \stackrel{\mathrm{r}}{B}) \underset{\mathrm{g}}{\mathrm{r}}{ }_{v} f_{\alpha}=0
$$




\section{The Vlasov equation conserves phase- space density: incompressible flow}

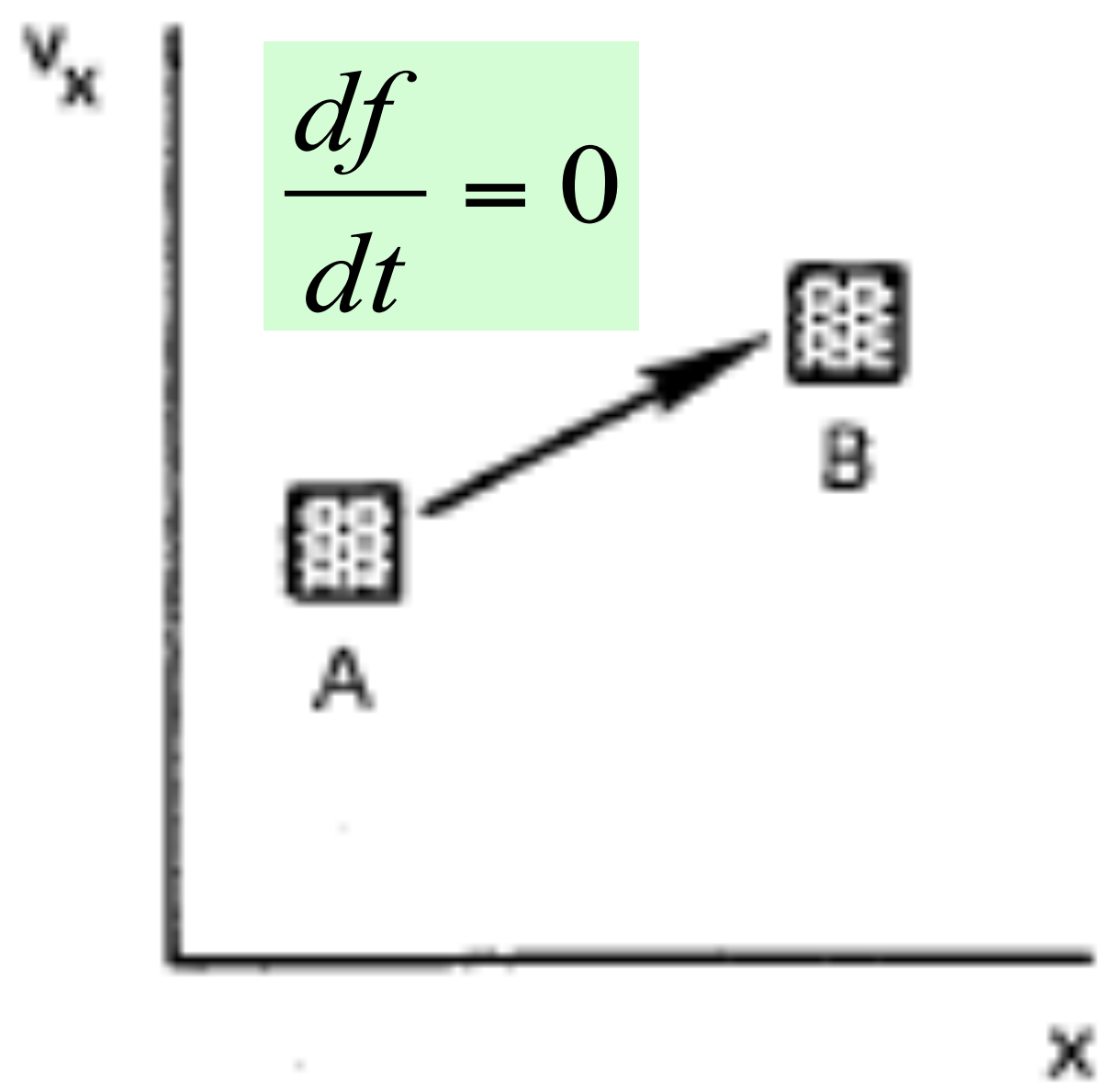

Figure: F.F. Chen, Introduction to Plasma Physics and Controlled Fusion, Vol. 1, $2^{\text {nd }}$ ed., Plenum Press, NY, 1984. 


\section{Derivation of Plasma Fluid Eqns}

1. The challenge of plasma physics

2. Kinetic equations

- Klimontovich, Boltzmann, Vlasov

- Distribution functions

3. Particle motion

4. Velocity moments of Boltzmann equation + approximations

=> fluid equations \& magnetohydrodynamics

5. Plasma equilibria, waves, instabilities, self-organization 


\section{Charged Particles Curve in a Magnetic Field}

Magnetic force on a

$$
\stackrel{1}{F}=q \stackrel{r}{v} \times \stackrel{1}{B}
$$

moving charged particle: $|\stackrel{\mathrm{r}}{F}|=q v B \sin \theta$

$>$ A magnetic field can change the velocity of a moving charged particle, but not its speed.

$>$ A moving particle's direction will be changed by a magnetic field if it has a velocity component perpendicular to the field. 


\section{Cyclotron Motion}

If a moving charged particle's trajectory is entirely within a uniform transverse magnetic field, its orbit is closed and circular.

$$
\frac{m v_{\perp}^{2}}{R}=F_{\perp}=q v_{\perp} B
$$

$\vec{B}$ out of plane

Orbital Period $T$ :

$$
T=\frac{2 \pi R}{v_{\perp}}=\frac{2 \pi}{v_{\perp}} \frac{m v_{\perp}}{q B}=\frac{2 \pi m}{q B}
$$

Orbital Frequency $f_{\mathrm{c}}: \quad f_{c}=\frac{1}{T}=\frac{q B}{2 \pi m}$ Frequency 


\section{Motion of a charged particle in an arbitrary direction relative to a magnetic field}

- If $\vec{v}$ is neither perpendicular nor parallel to $\vec{B}$, a helical (spiral) motion about the direction of $B$ results.

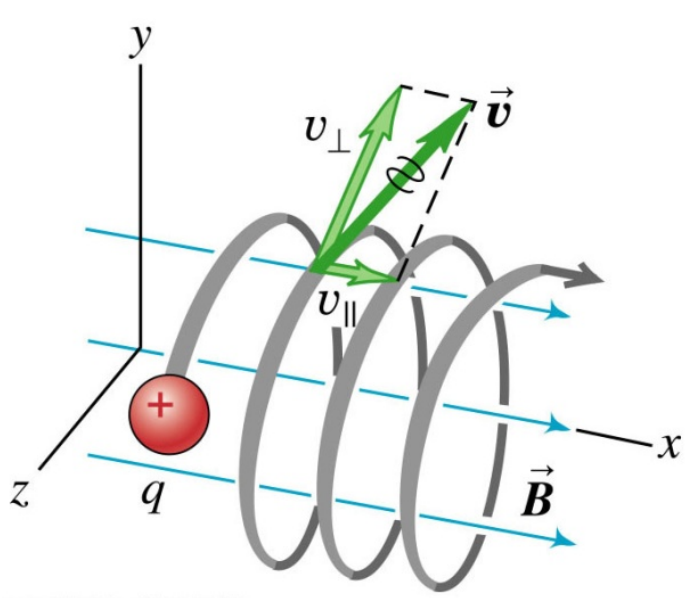

- Such motion of positive ions from the solar wind ionizes the atmosphere and is responsible for the phenomenon of the Aurora Borealis (northern lights)

Charged particle

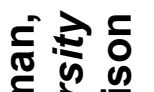

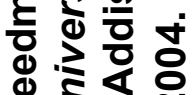

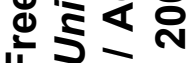
的页发 要 क्ष की 穴 $\frac{\pi}{2}$

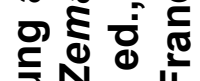
柆 웧ㄷㄷ ด ธิ่ I y 创 宛

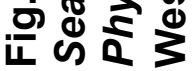

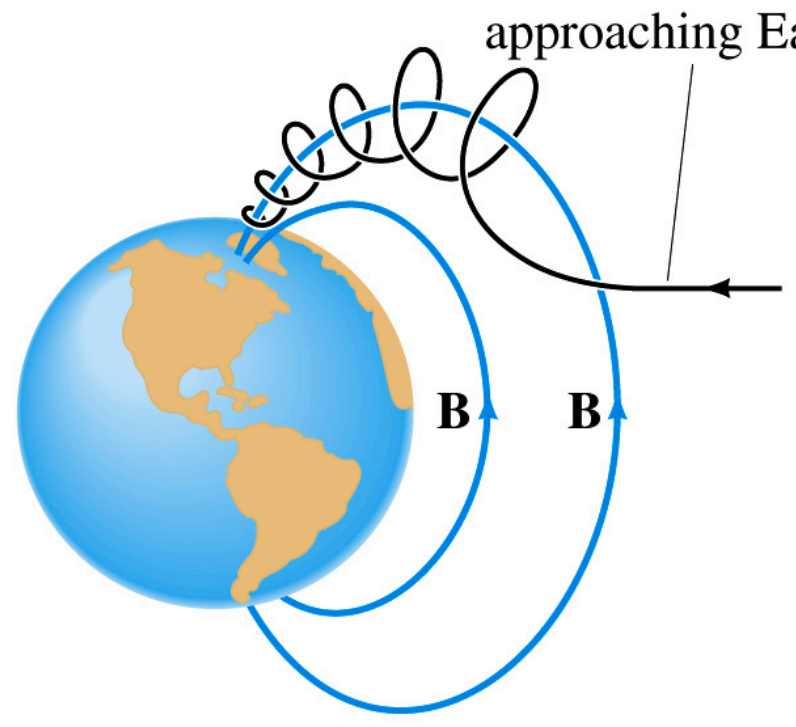

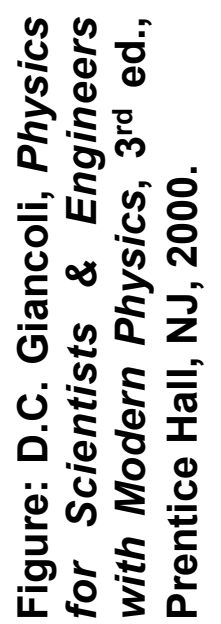




\section{Solar storms make the news}

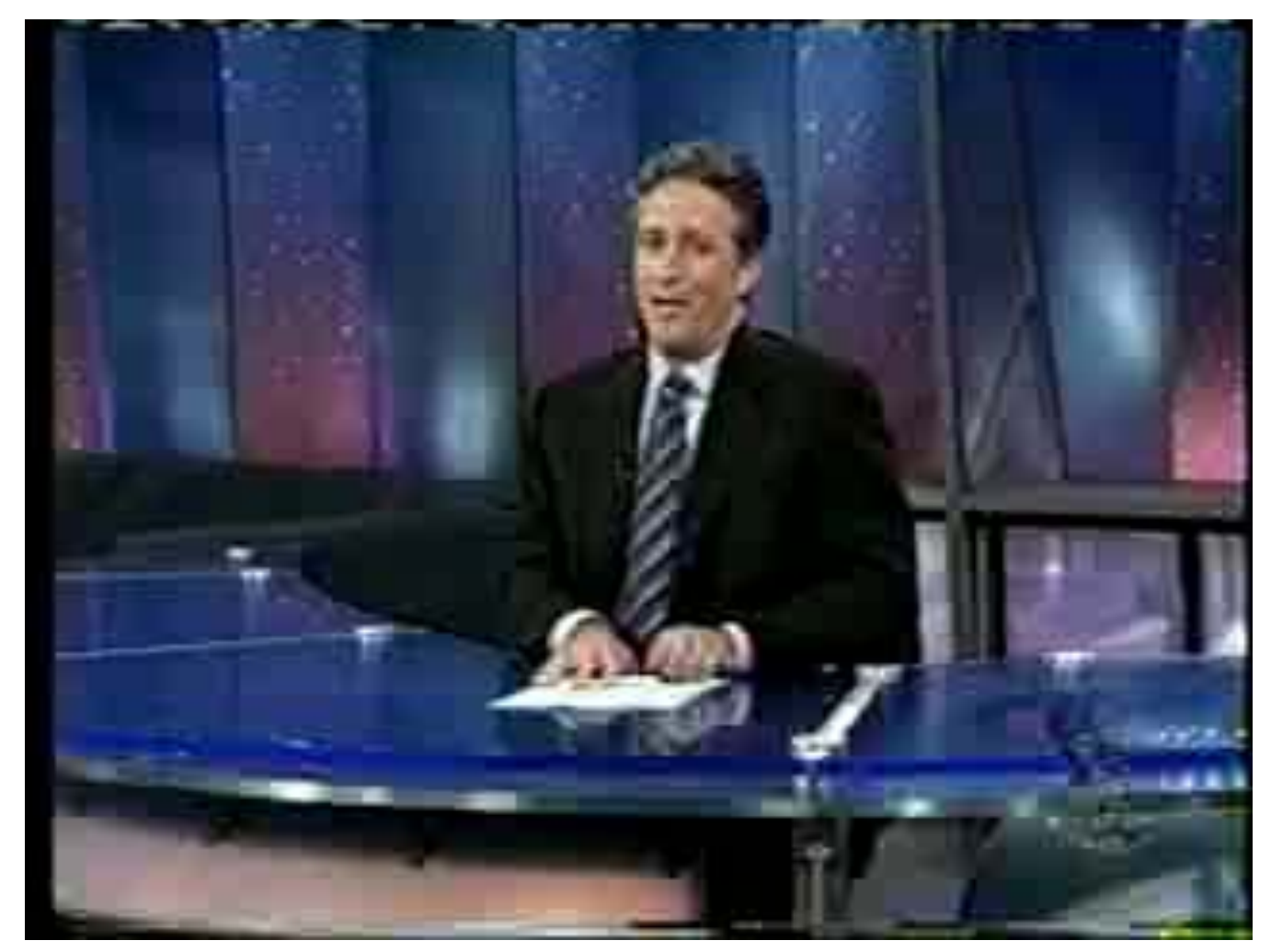

The Daily Show with Jon Stewart Comedy Central, 2004 


\section{A “Magnetic Bottle” for Charged Particles}

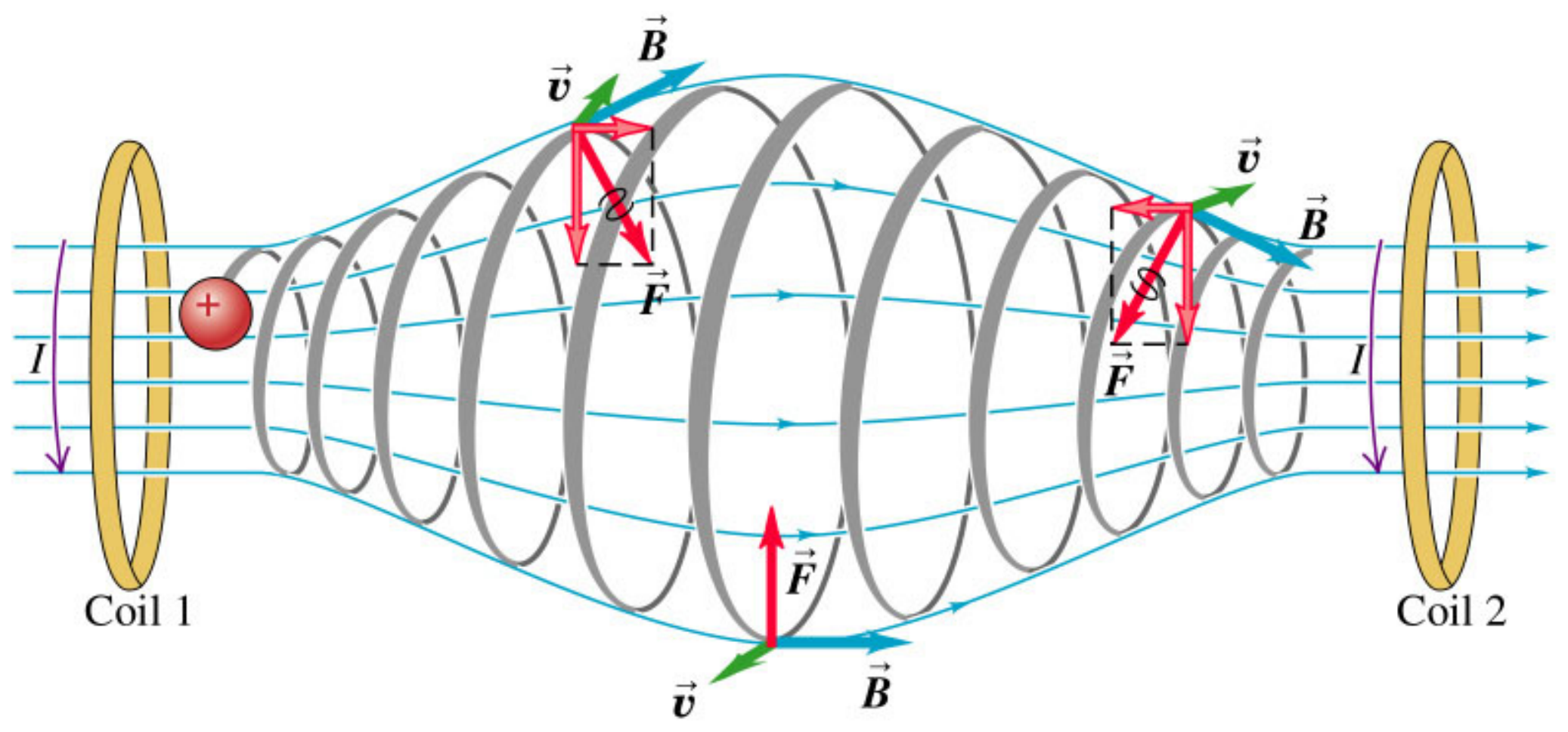

Magnetic fields are used to confine the charged particles in hot ionized plasmas (e.g., in fusion energy research). 


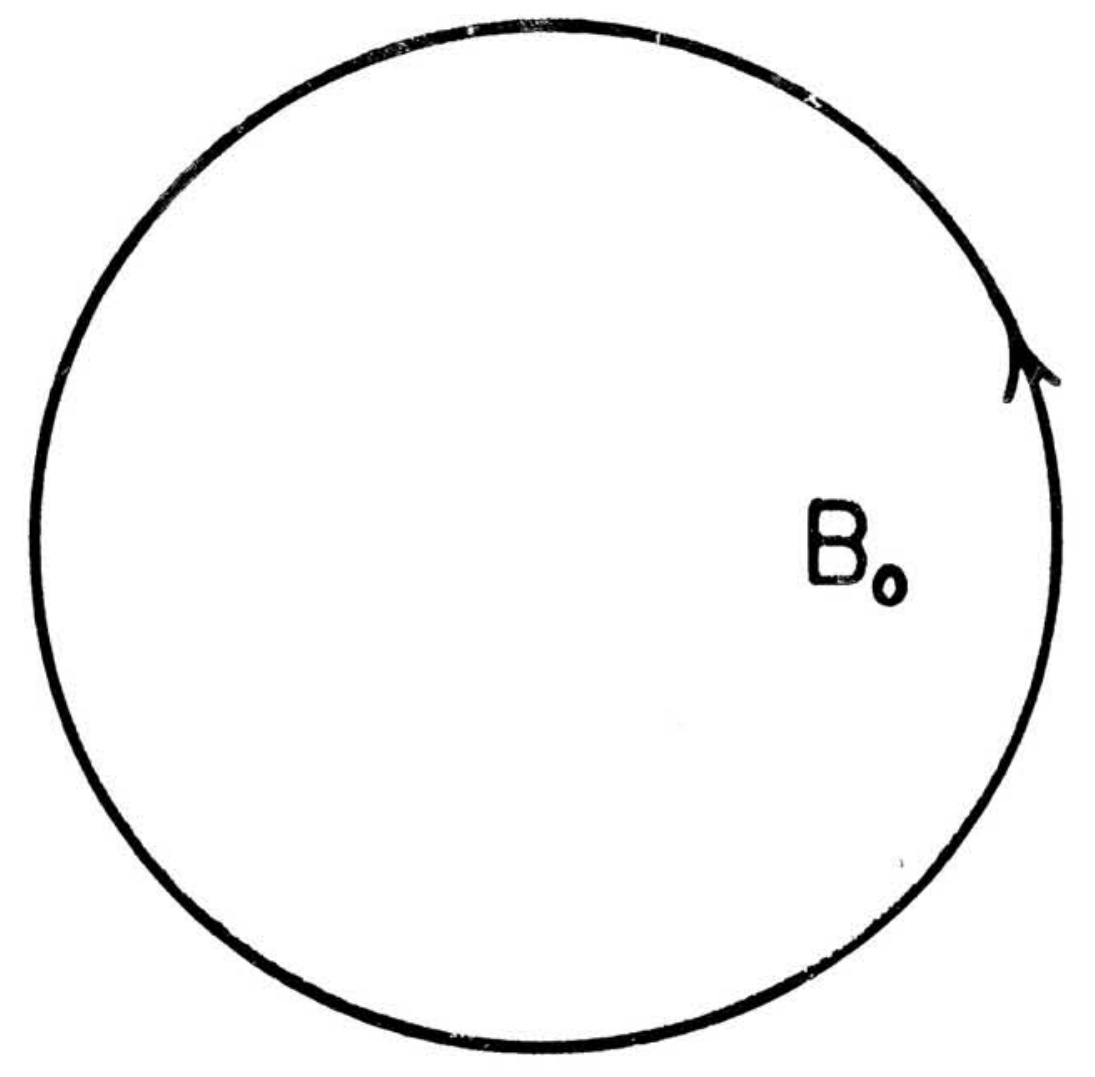

\section{Magnetic \\ Confinement \\ Device}

Cartoon:

ZT-H, LANL

ZT-H 4MA Reversed Field Pinch

THEORICAL PHYSICSTS' DESIGI!

(Lowesf Crder Only) 
1953: Perhapsatron, LANL

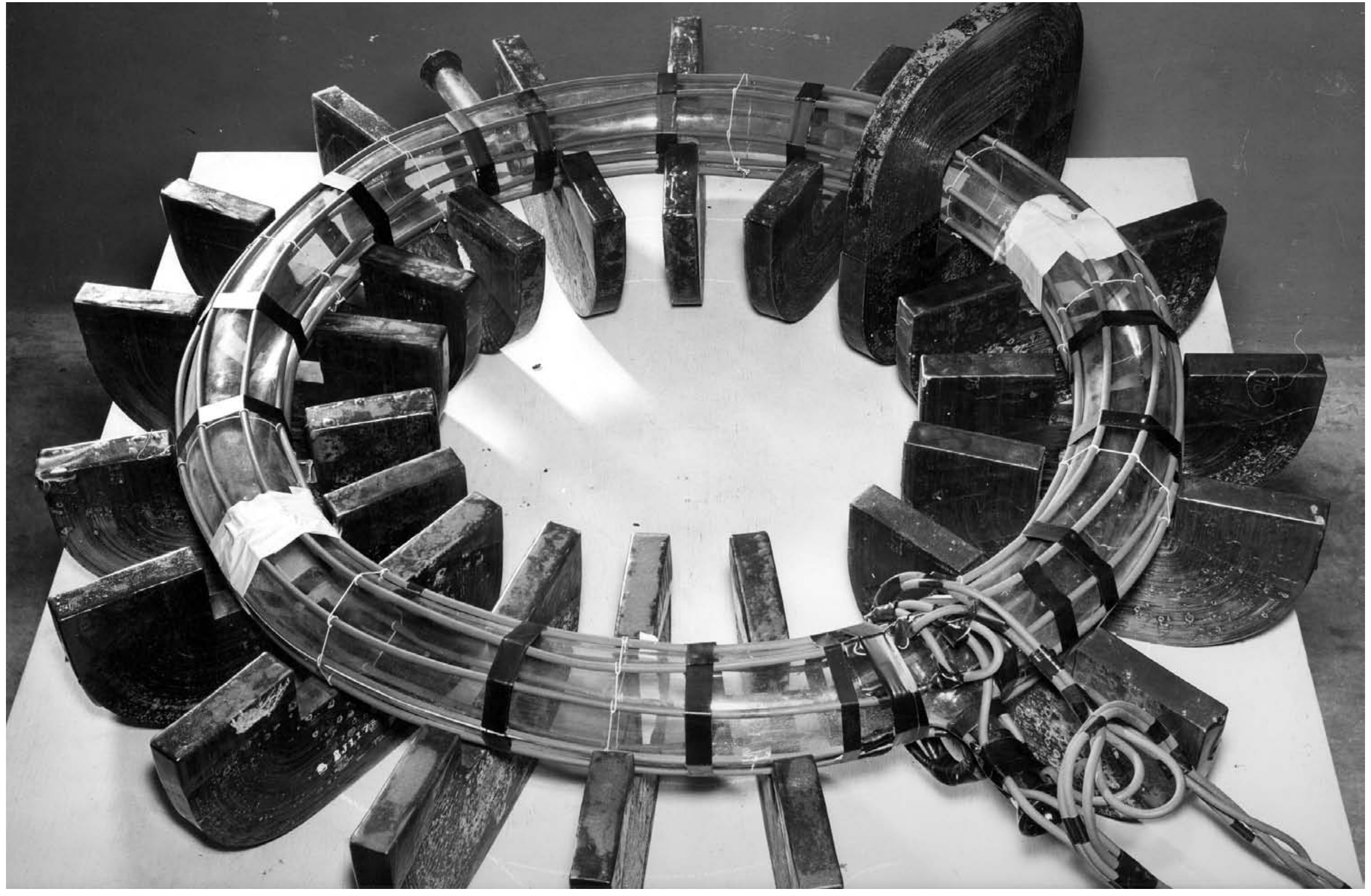



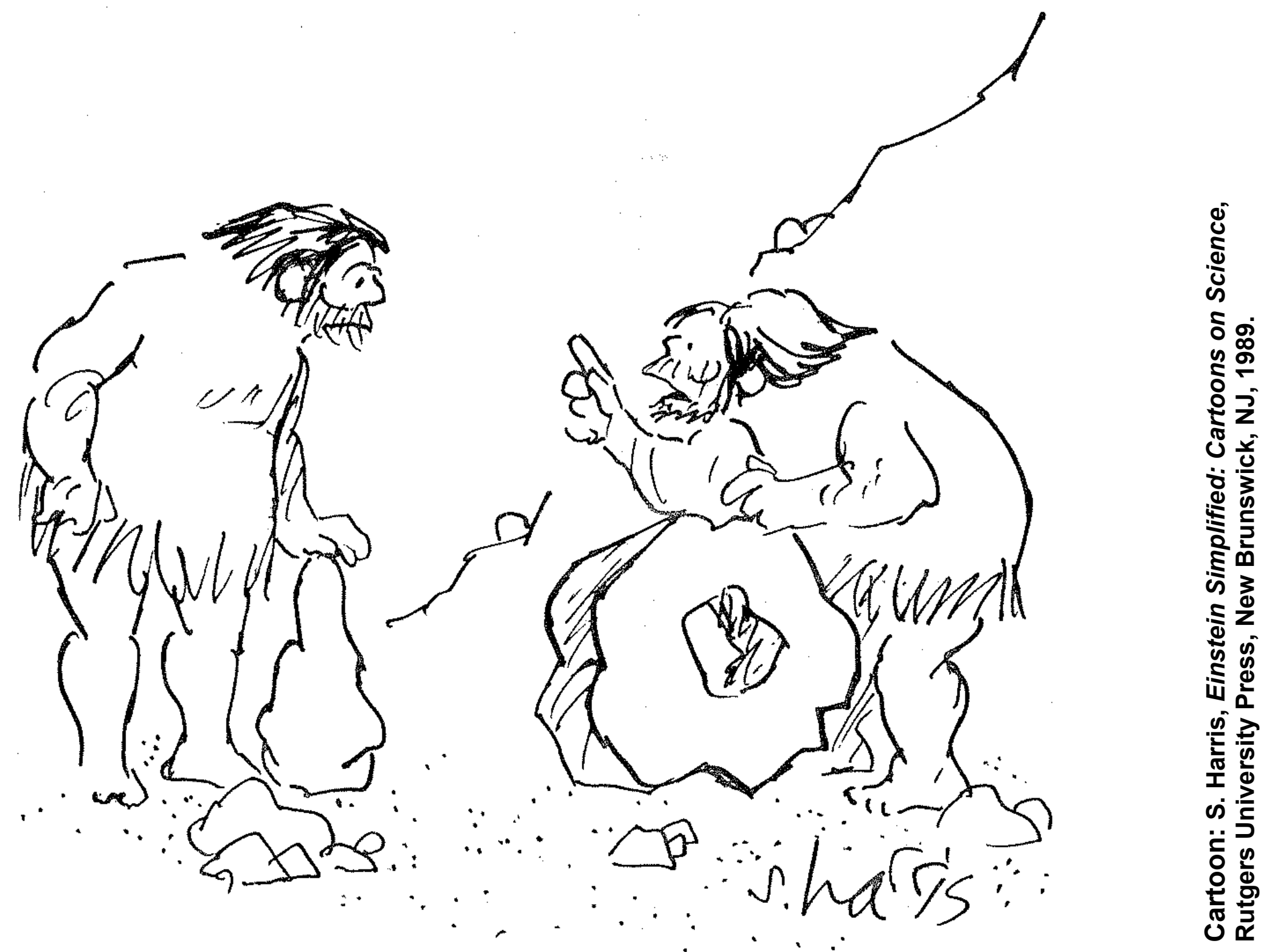

"IT MAY NOT BE A PERFECT WHEEL, BUT ITS A STATE-OF-THE-ART WHEEL."

BSB 6/20/12 


\section{Achieving a long $\tau_{\mathrm{E}} \sim 1 \mathrm{~s}$ requires a large $(10 \mathrm{~m})$ plasma}

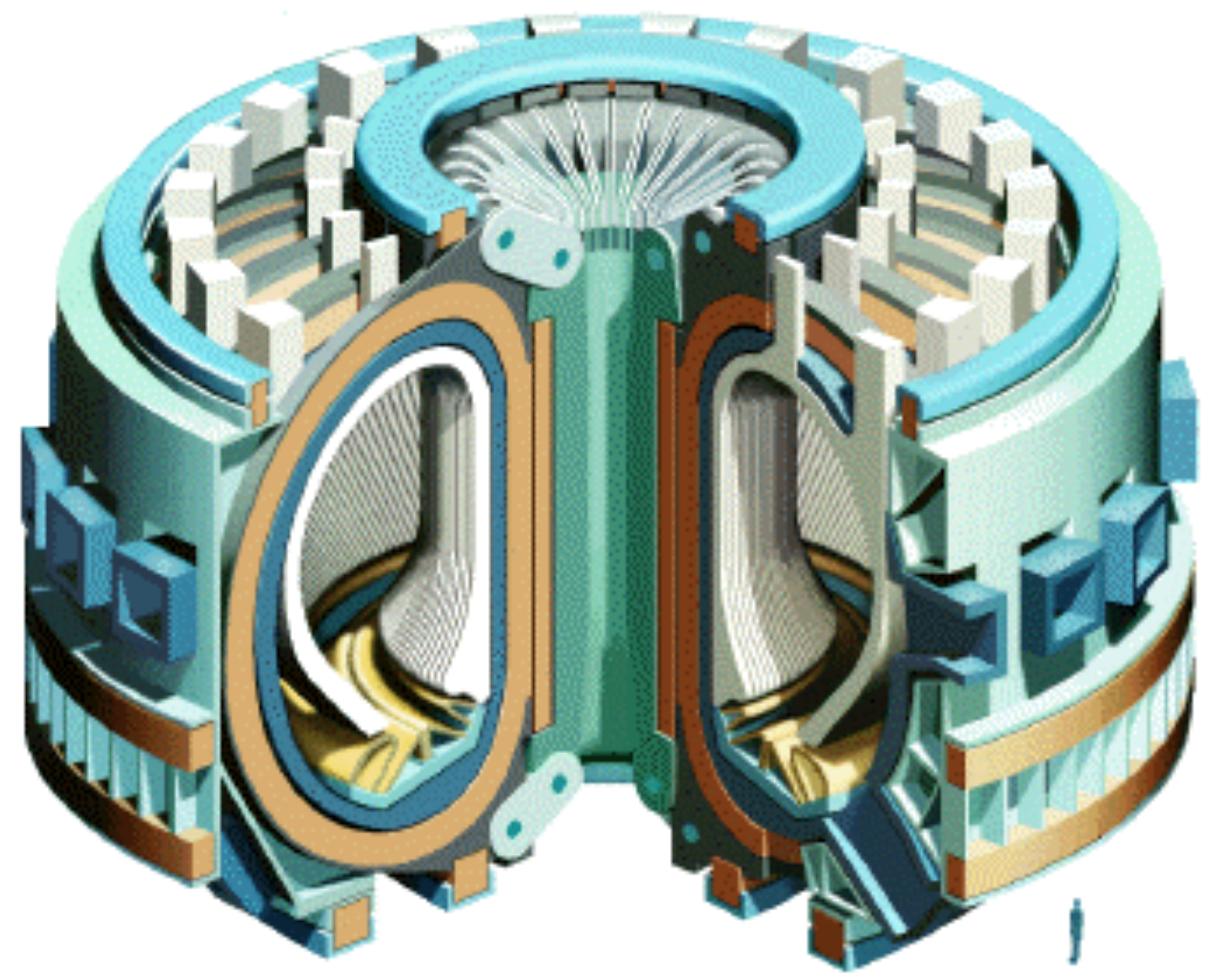

Figure: ITER, www.iter.org

$$
\int_{N^{2}}^{T_{2}}
$$

International

Thermonuclear

Experimental Reactor (ITER)

$500 \mathrm{MW}$

400 seconds

Gain Q>10

\$21 billion

(July 2010) 


\section{$E$ perpendicular to $B$ makes a charged particle drift sideways}

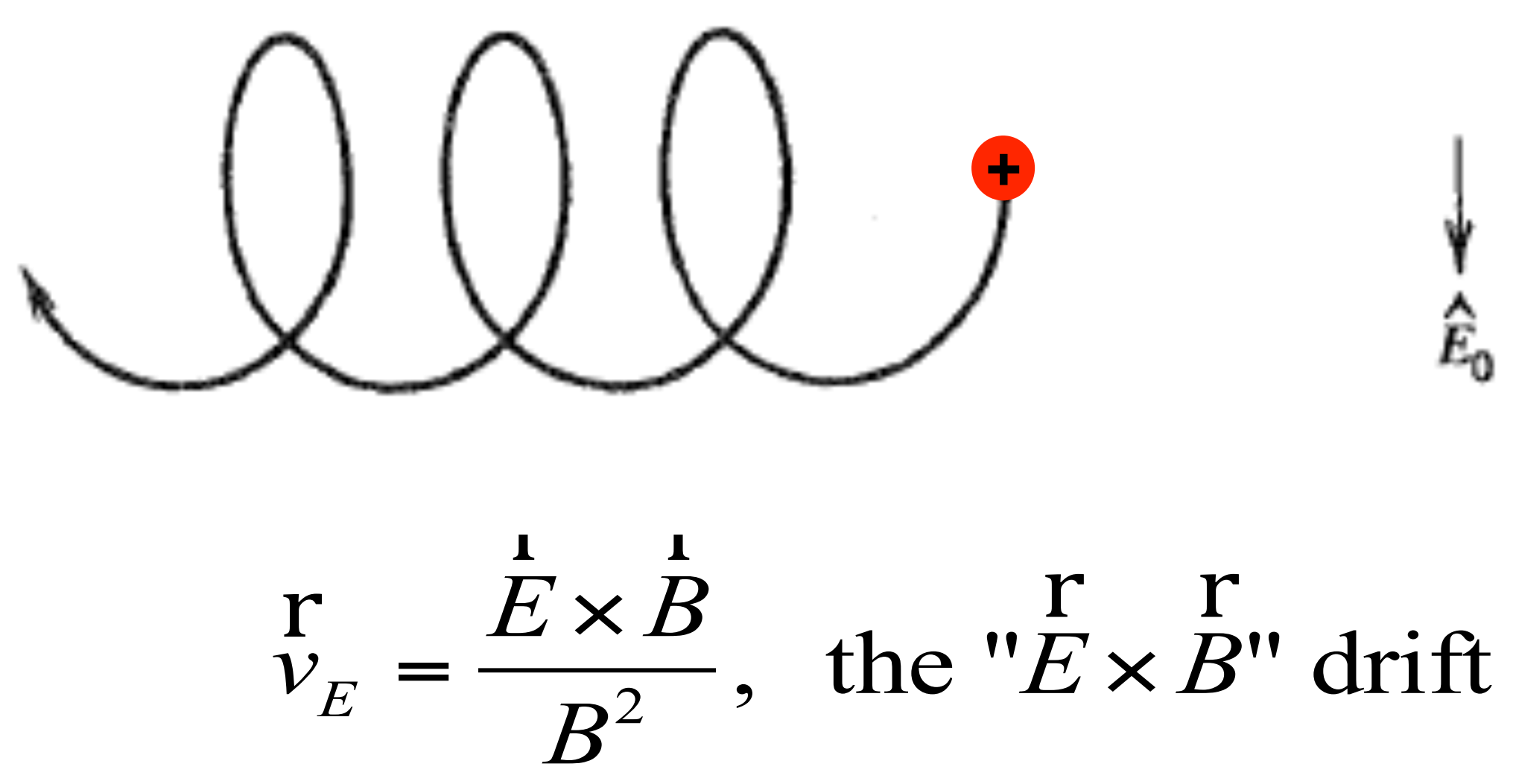

$\odot \hat{B}_{0}$

Figure: D.R. Nicholson, Introduction to Plasma Theory, Wiley, NY, 1983. 


\section{Bonus Quiz}

An electron in vacuum is released from rest at a location near the Earth's surface where $E=0$, $B=0.5 \mathrm{G}$ north, and $g=9.8 \mathrm{~m} / \mathrm{s}^{2}$ down. Describe and sketch the motion of the electron.

$\triangle 4$

West

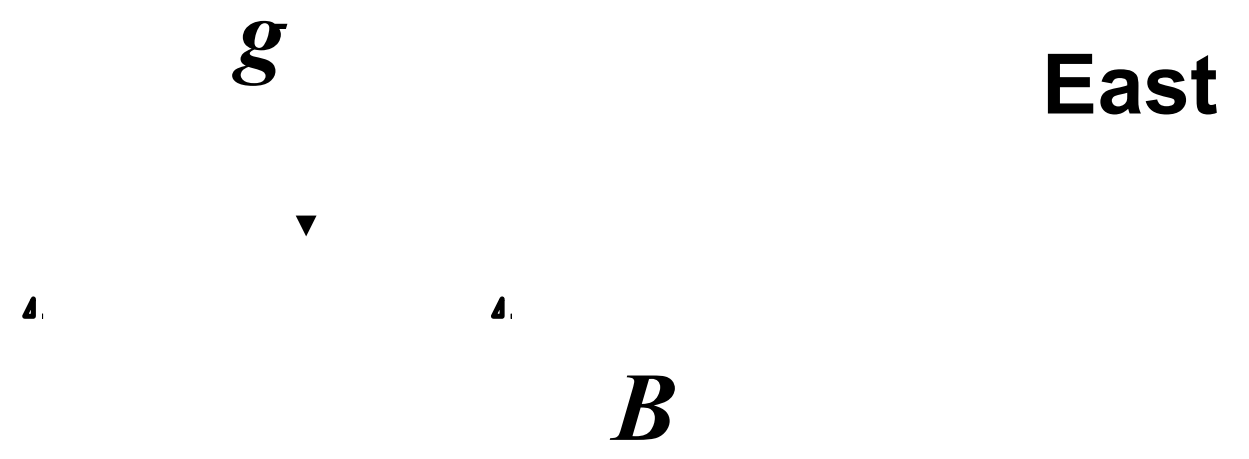




\section{The electron makes a cusp motion, falling then rising, drifting west}

This image is not quite

g correct: because $v(0)=0$,

$\Delta$

1 the tops of the orbit .

have radius $r=0$,

i.e., they are points

beverererevere $\Theta$

$\stackrel{\odot}{\circ} 0$

ION

B

$\otimes \mathbf{B}$

ELECTRON

West

East

Figure: F.F. Chen, Introduction to Plasma Physics and

Controlled Fusion, Vol. 1, $2^{\text {nd }}$ ed., Plenum Press, NY, 1984. 


\section{Derivation of Plasma Fluid Eqns}

1. The challenge of plasma physics

2. Kinetic equations

- Klimontovich, Boltzmann, Vlasov

- Distribution functions

3. Particle motion

4. Velocity moments of Boltzmann equation + approximations

=> fluid equations $\&$ magnetohydrodynamics

5. Plasma equilibria, waves, instabilities, self-organization 


\section{Integrate $f\left(x, y, z, v_{x}, v_{y}, v_{z}\right)$ over 3D velocity space to obtain density \& other fluid quantities}

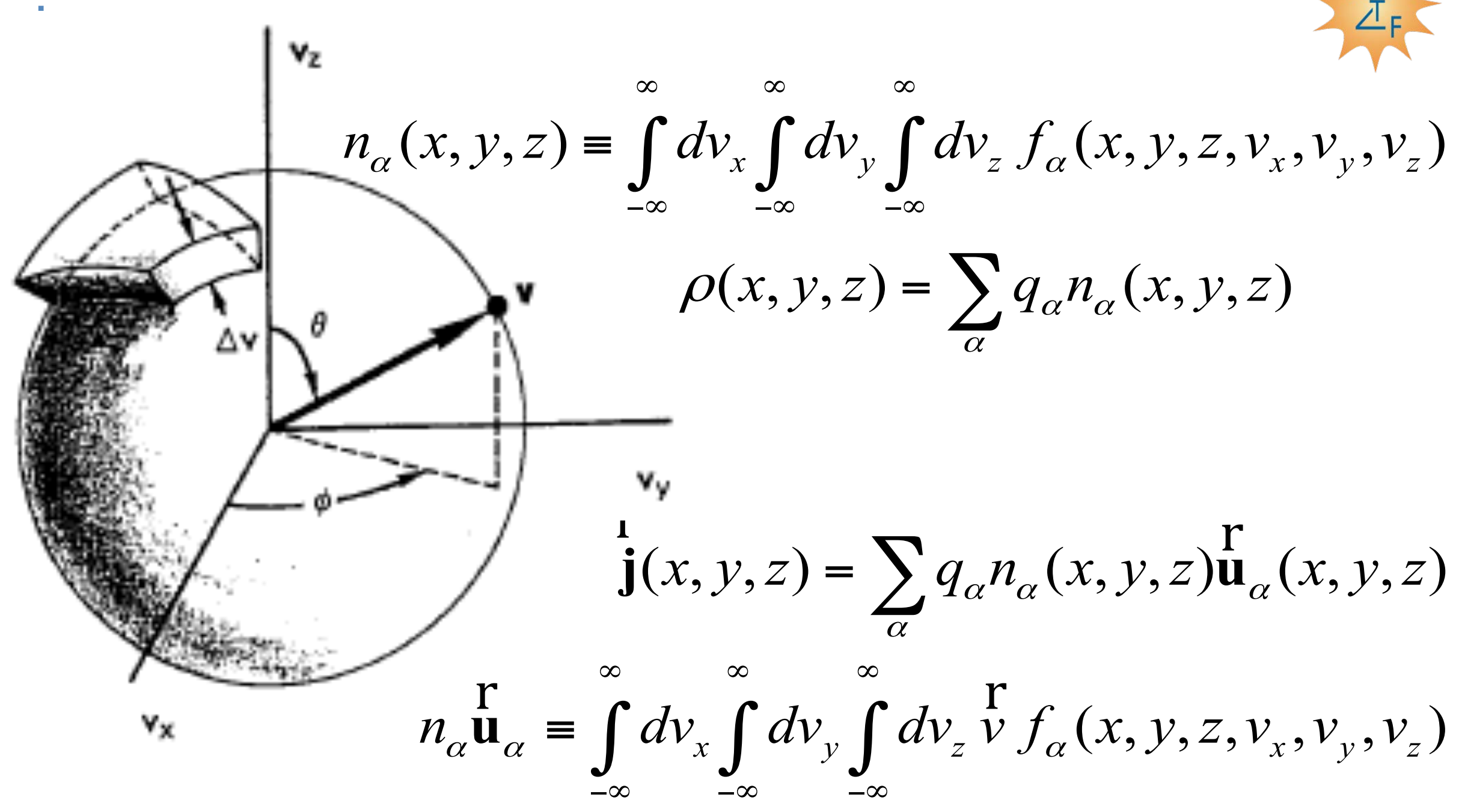

Figure: F.F. Chen, Introduction to Plasma Physics and Controlled Fusion, Vol. 1, $2^{\text {nd }}$ ed., Plenum Press, NY, 1984. 


\section{Integrate Boltzmann eqn to obtain fluid eqns that capture the main plasma collective properties}

\section{For each species $\alpha$ :}

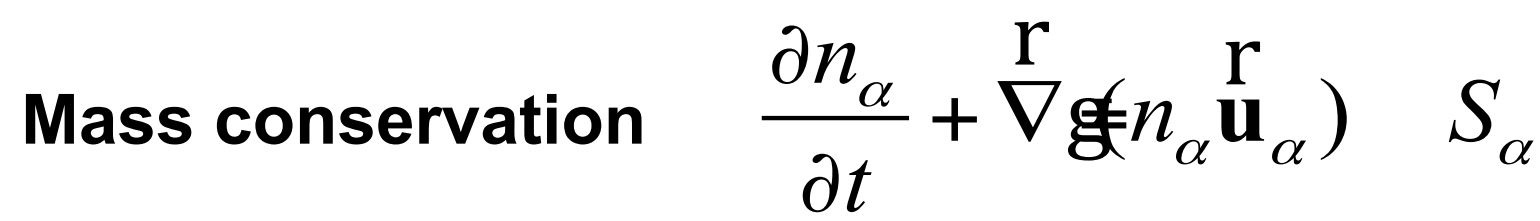

Momentum conservation

$$
m_{\alpha} n_{\alpha} \frac{d \dot{\mathbf{u}}_{\alpha}}{d t}=n_{\alpha} q_{\alpha}\left[\stackrel{\mathrm{r}}{\mathbf{E}}+\stackrel{\mathrm{r}}{\mathbf{u}_{\alpha}} \times \stackrel{\mathrm{r}}{\mathbf{B}}\right]-\stackrel{\mathrm{r}}{\nabla} \mathbf{g}_{\alpha}-m_{\alpha} S_{\alpha} \mathbf{u}_{\alpha}+\sum_{\beta} \stackrel{\mathrm{r}}{\mathbf{R}}_{\alpha \beta}
$$

$>$ Convective derivative:

$$
\frac{d}{d t}=\frac{\partial}{\partial t}+\underset{\mathbf{u}_{\alpha}}{\mathrm{g}} \mathrm{r}
$$

$>$ Resistive drag force density: $\stackrel{\mathfrak{R}}{\mathbf{R}}_{\alpha \beta}=-m_{\alpha} n_{\alpha} v_{\alpha \beta}\left(\begin{array}{lll}\mathbf{r} & \mathbf{r} \\ \mathbf{u}_{\alpha} & -\mathbf{\sharp}_{\beta}\end{array}\right) \quad \stackrel{\mathfrak{l}}{\mathbf{R}}_{\beta \alpha}$

Energy conservation: Heat equation

$$
\begin{aligned}
& \mathbf{P}=\mathbf{I} p \\
& p=n T=C n^{\gamma}
\end{aligned}
$$

$\rightarrow$ simplest to use Equation of State 
With further approximations, obtain single fluid magnetohydrodynamics (MHD), describing an electrically conducting fluid in the presence of magnetic field

Slow $\quad \tau>>\frac{1}{v_{\text {collision }}}, \frac{L}{C} \quad$ Big $L>>\lambda_{\text {mean freepath }}, \lambda_{\mathrm{De}}, r_{\mathrm{L}}$

Quasi-neutral $\quad n_{\mathrm{e}} ; n_{\mathrm{i}} Z \quad$ Thermal equilibrium $T_{\mathrm{e}} ; T_{\mathrm{i}}$

Equations:

Mass Continuity

$\left.\frac{\mathrm{d} \rho}{\mathrm{d} t}+\stackrel{\mathrm{r}}{\mathrm{f}} \mathrm{f} \rho \mathrm{r}\right) \quad 0$

Momentum Equation

$\rho \frac{\mathrm{d} \dot{\mathrm{u}}}{\mathrm{d} t}=\stackrel{\mathrm{r}}{j} \times \stackrel{\mathrm{r}}{B}-\stackrel{\mathrm{r}}{\nabla} p+\rho \stackrel{\mathrm{r}}{\mathrm{r}}$

Equation of state

$\frac{\mathrm{d}}{\mathrm{d} t}\left(\frac{p}{\rho^{\gamma}}\right)=0, \quad p=\frac{\rho T}{M}(Z+1)$

Ampere's Law

$\stackrel{1}{\nabla} \times B^{1}=\mu_{0} \stackrel{1}{j} \Rightarrow \stackrel{1}{\nabla} \dot{I} j \quad 0$

Faraday Law

$\stackrel{\mathrm{r}}{\nabla} \times \underset{E}{\mathrm{r}}=-\frac{\partial B}{\partial t}, \quad \stackrel{\mathrm{r}}{\nabla \mathrm{g} B}$

Ohm's Law

$\stackrel{1}{E}+\stackrel{\mathrm{r}}{u} \times \stackrel{1}{B}=\stackrel{\stackrel{r}{j}}{j}$ 


\section{Derivation of Plasma Fluid Eqns}

1. The challenge of plasma physics

2. Kinetic equations

- Klimontovich, Boltzmann, Vlasov

- Distribution functions

3. Particle motion

4. Velocity moments of Boltzmann equation + approximations

=> fluid equations \& magnetohydrodynamics

5. Plasma equilibria, waves, instabilities, self-organization 


\section{Plasmas are complex systems}

$>$ Plasmas can exhibit self-organized behavior of high complexity

$>$ Self-organization occurs in many areas

* Space \& astrophysics, biosystems, micro- and nano- components, protein folding

- Selective decay processes, thermodynamics

- Dissipation of some quantity on small scales (e.g., energy in eddies, turbulence)

- Persistence of other quantities on larger spatial scales (e.g., helicity) 


\section{Example of self-organization (numerical simulation): reorganization of plasma pressure into stable profile}

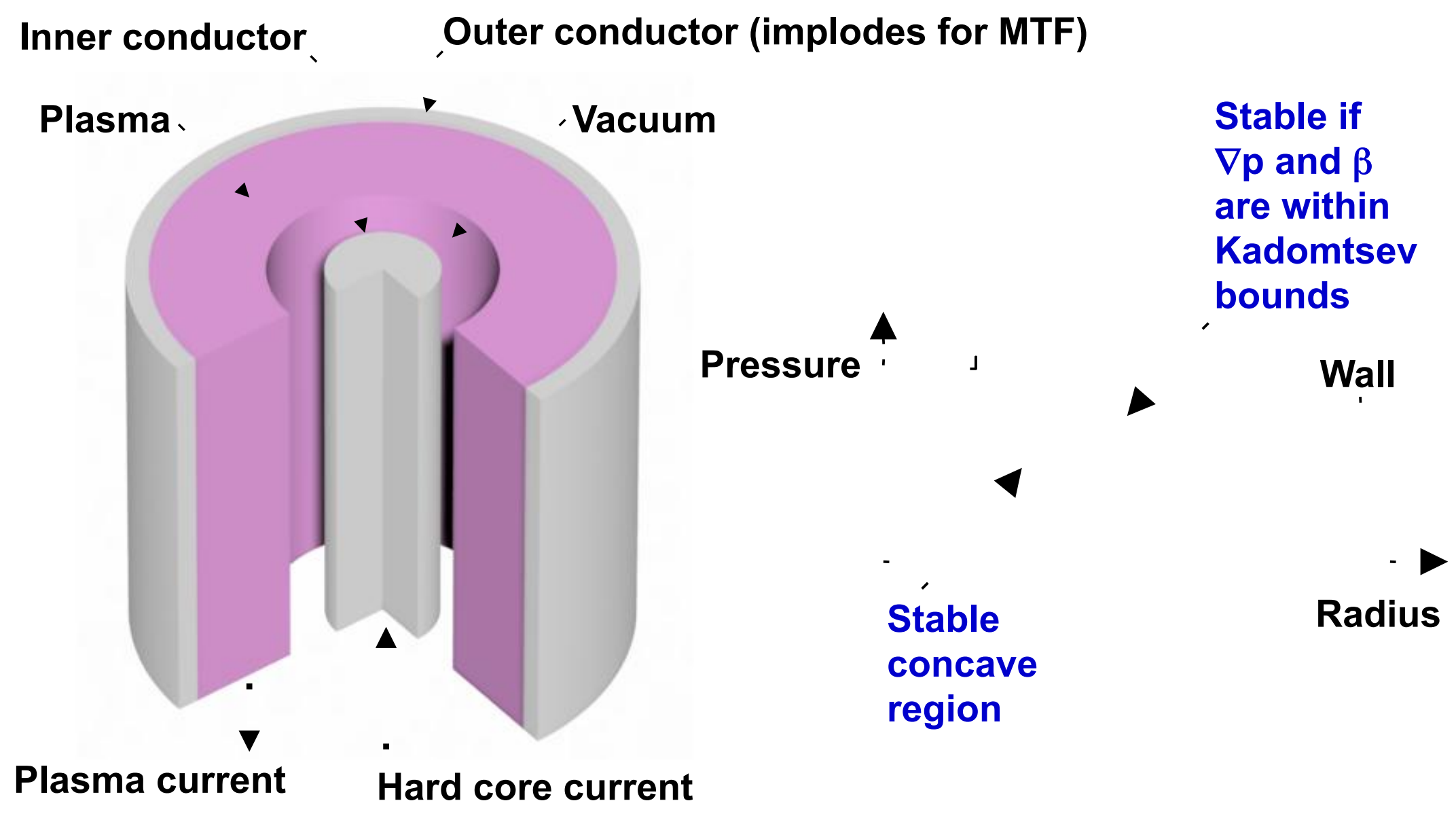




\section{Movie of dynamic formation phase}
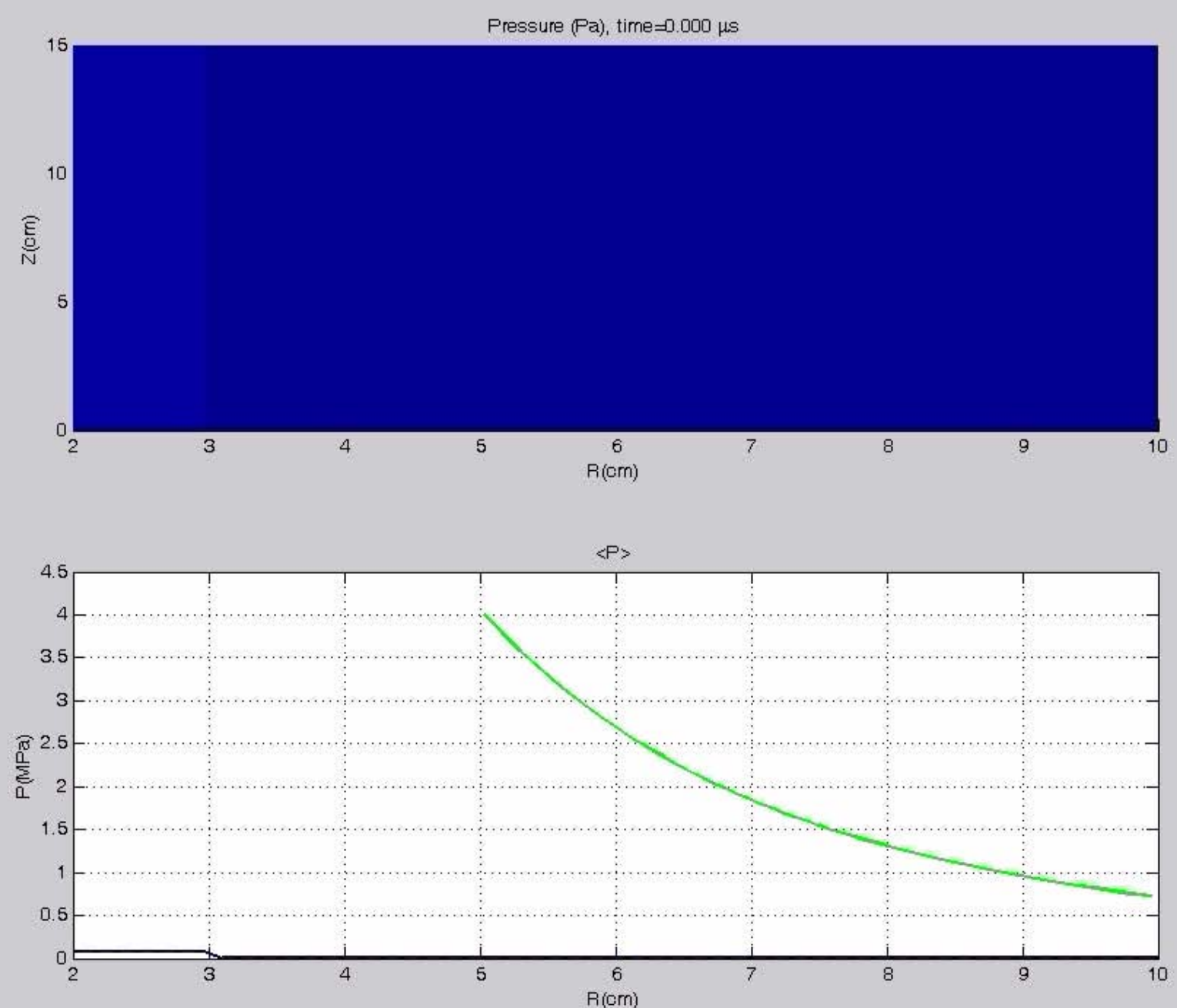


\section{Movie of equilibration phase}
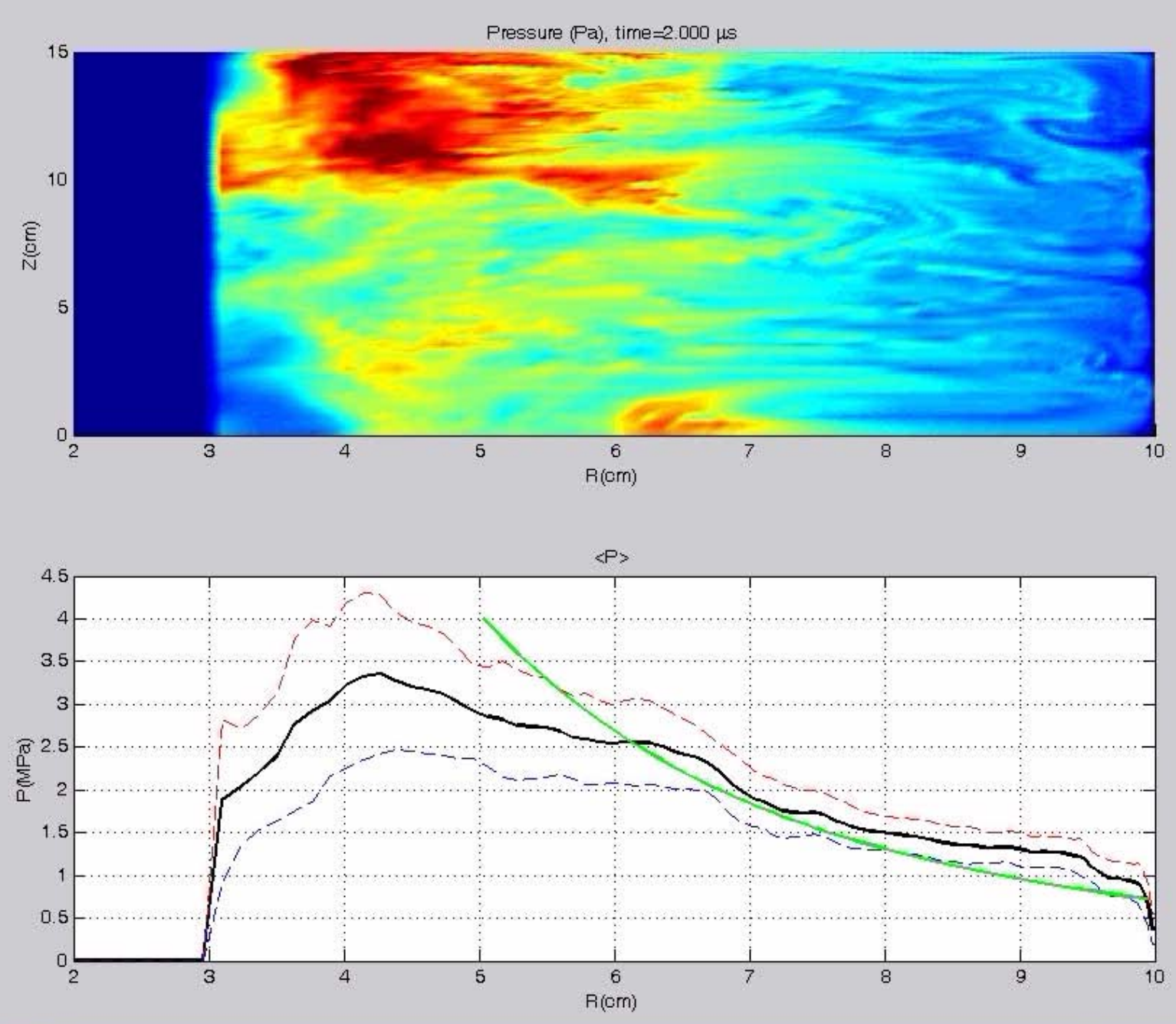


\section{Simulated plasma finds Kadomtsev}

marginally stable profile

$p, \mathrm{MPa}$

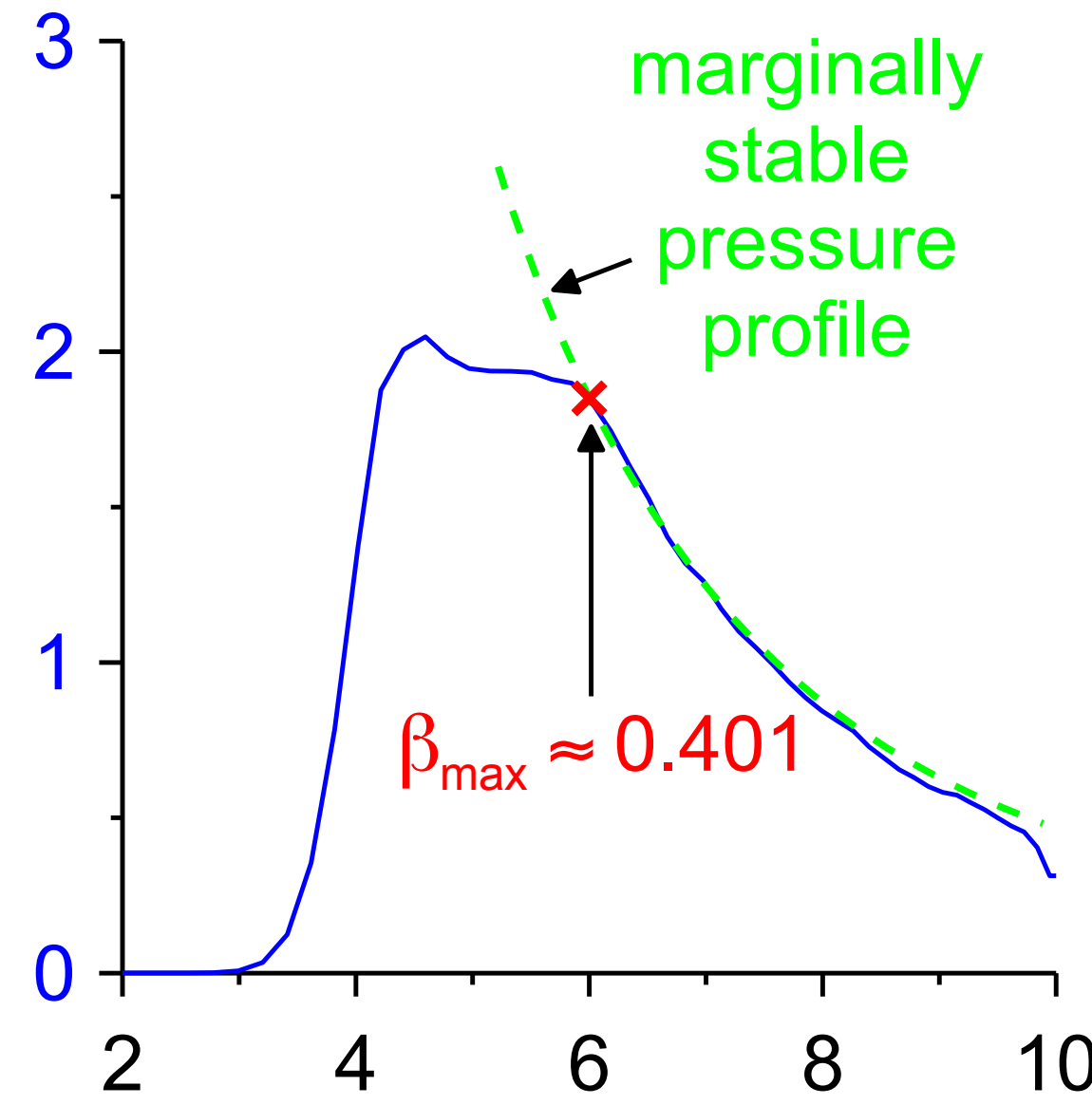

Point marked with " $x$ " is where beta for simulated profile is matched to Kadomtsev beta

Through $\mathrm{m}=\mathbf{0}$ turbulence, the plasma organizes itself into the marginally stable Kadomtsev state 
FRX-L: The Field Reversed Configuration (FRC) Plasma Injector for MTF
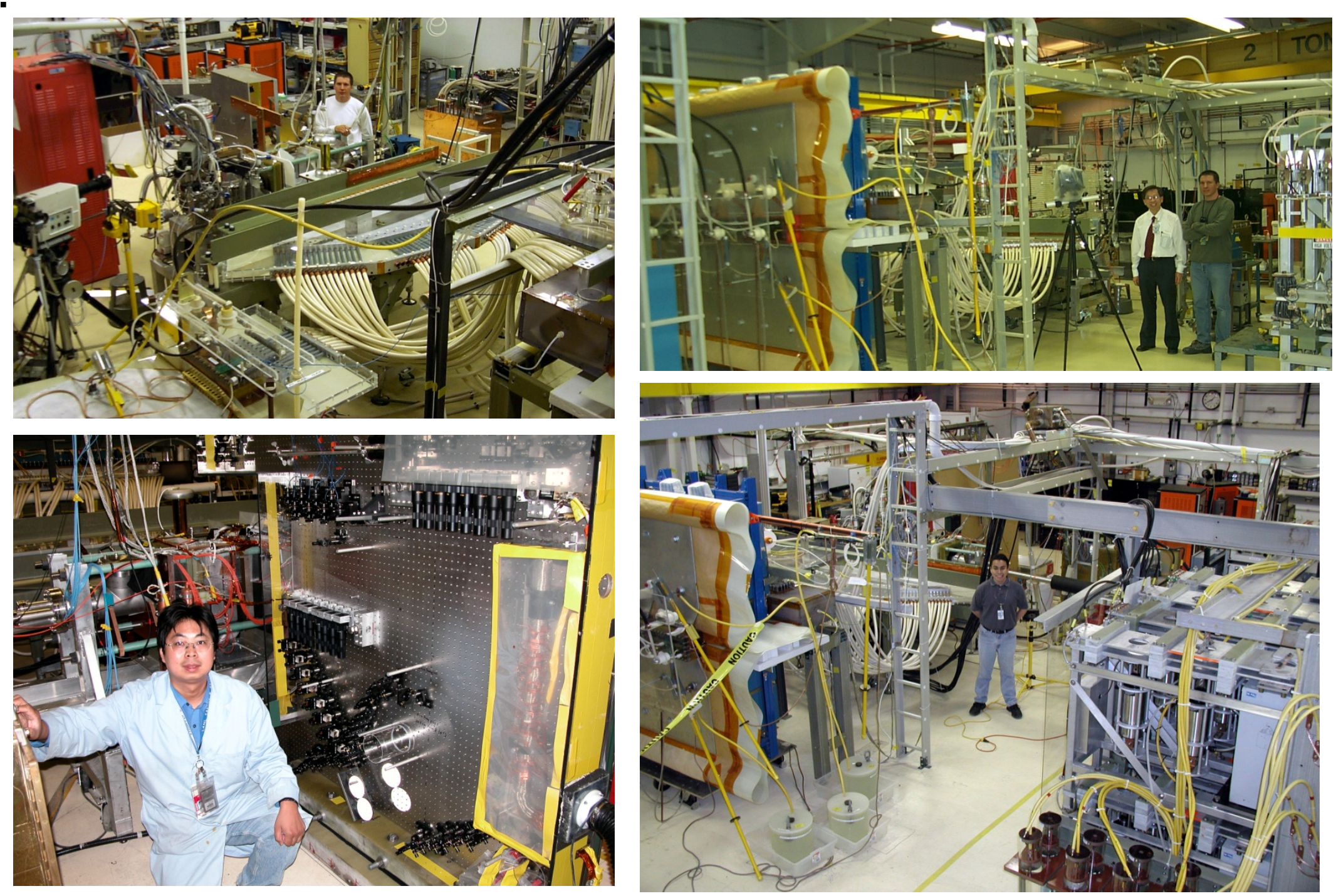

NISG .

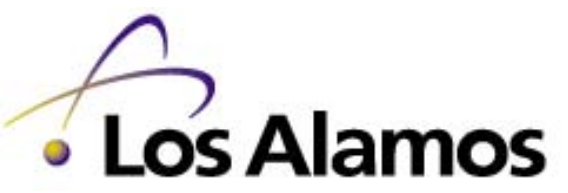




\section{Field Reversed Configuration high- $\beta$ self-organized plasma}

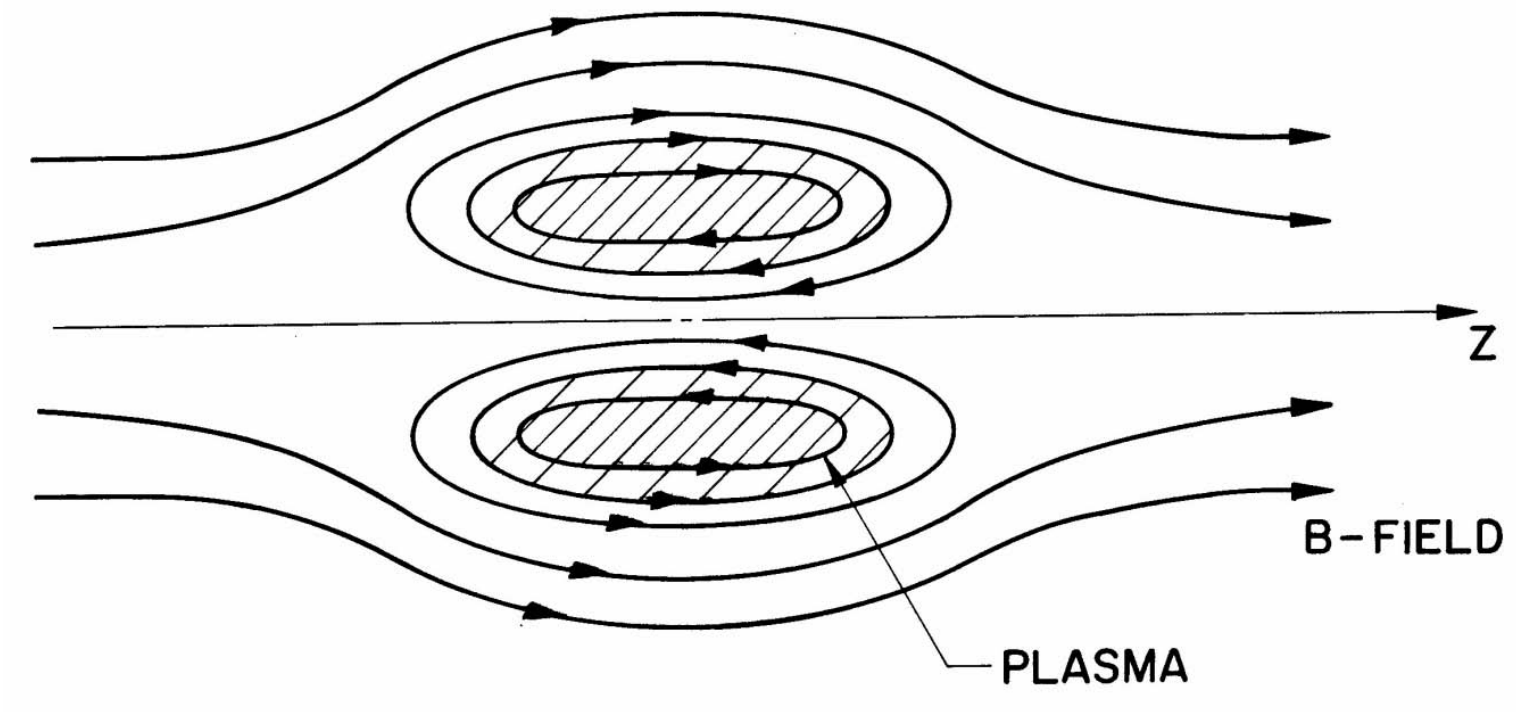

- $<\beta>\sim 1$

- compact torus like spheromak

- Can translate into liner

The LANL FRC has parameters orders of magnitude different than previous FRCs. How will FRC behave under compression? How will liner interact with FRC? 
Experiment on 1-MA/100-ns Zebra (UNR) studies plasma formed by multi-MG field on aluminum
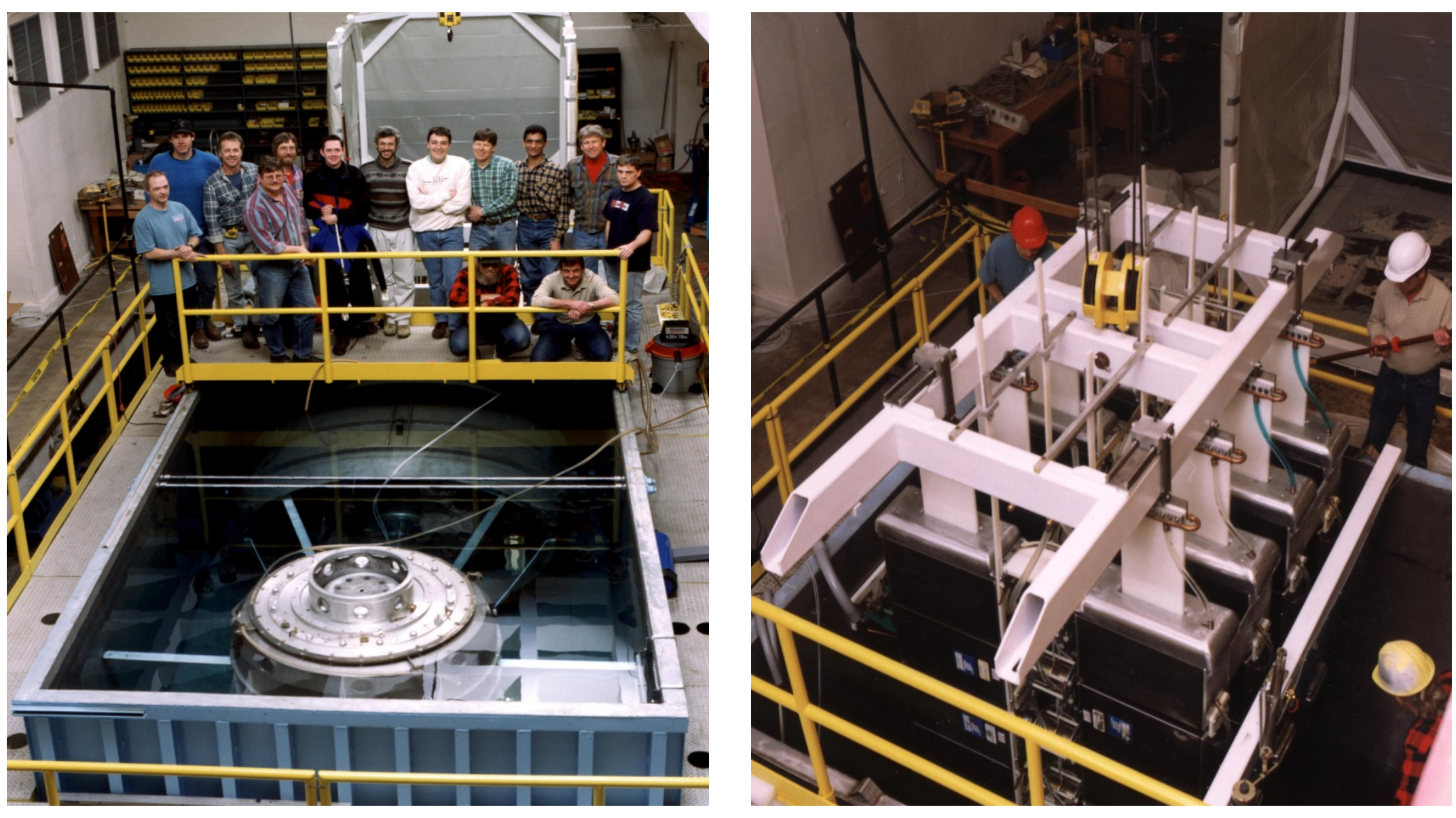


\section{Zebra Megagauss Experiment}

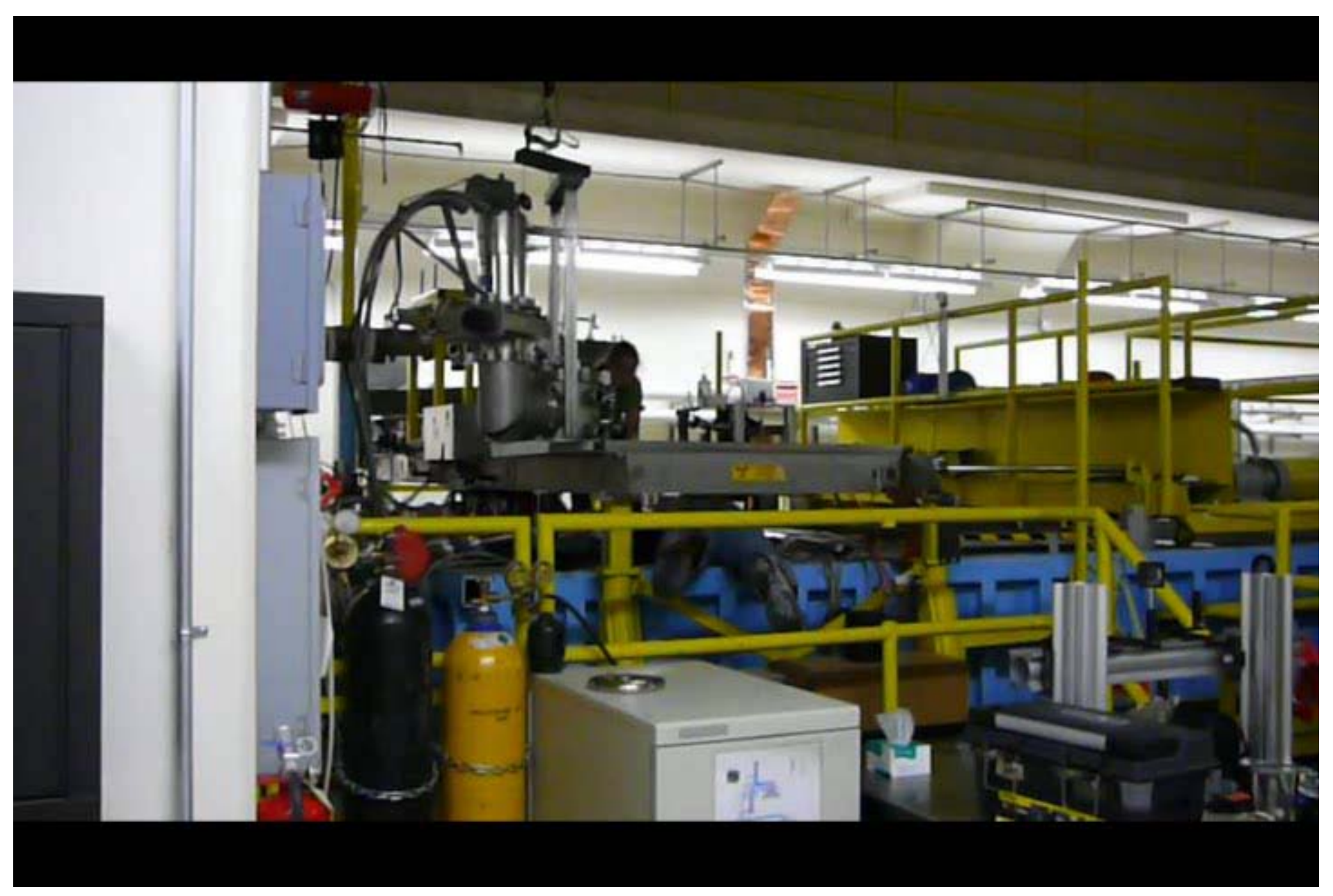

Movie: Stephan Fuelling, UNR, Oct 2006 


\section{Some good plasma references}

$\checkmark$ P.M. Bellan, Fundamentals of Plasma Physics, Cambridge University Press, Cambridge, United Kingdom, 2006.

$\checkmark$ J.A. Bittencourt, Fundamentals of Plasma Physics, $3^{\text {rd }}$ ed., Springer Science, New York, NY, 2004.

$\checkmark$ F.F. Chen, Introduction to Plasma Physics and Controlled Fusion, Vol. 1: Plasma Physics, ${ }^{\text {nd }}$ ed., Plenum Press, New York, NY, 1984.

$\checkmark$ R.P. Drake, High-Energy-Density Physics: Fundamentals, Inertial Fusion and Experimental Astrophysics, Springer Verlag, New York, NY, 2006.

$\checkmark$ R.J. Goldston and P.H. Rutherford, Introduction to Plasma Physics, Institute of Physics Publishing, Philadelphia, PA, 1995.

$\checkmark$ D.R. Nicholson, Introduction to Plasma Theory, John Wiley \& Sons, New York, NY, 1983. 


\section{Thanks!}
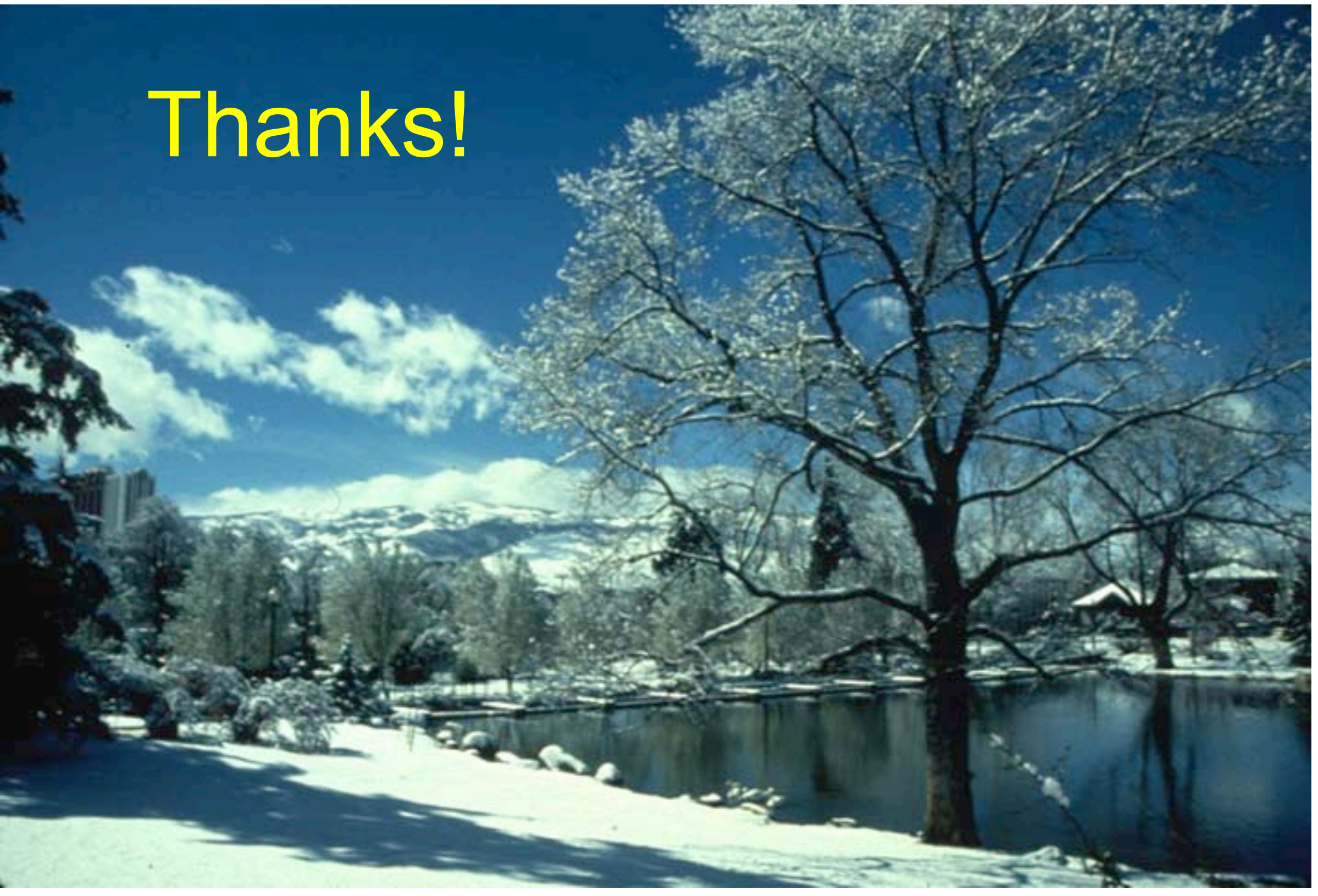

Photo: University of Nevada, Reno, www.unr.edu 


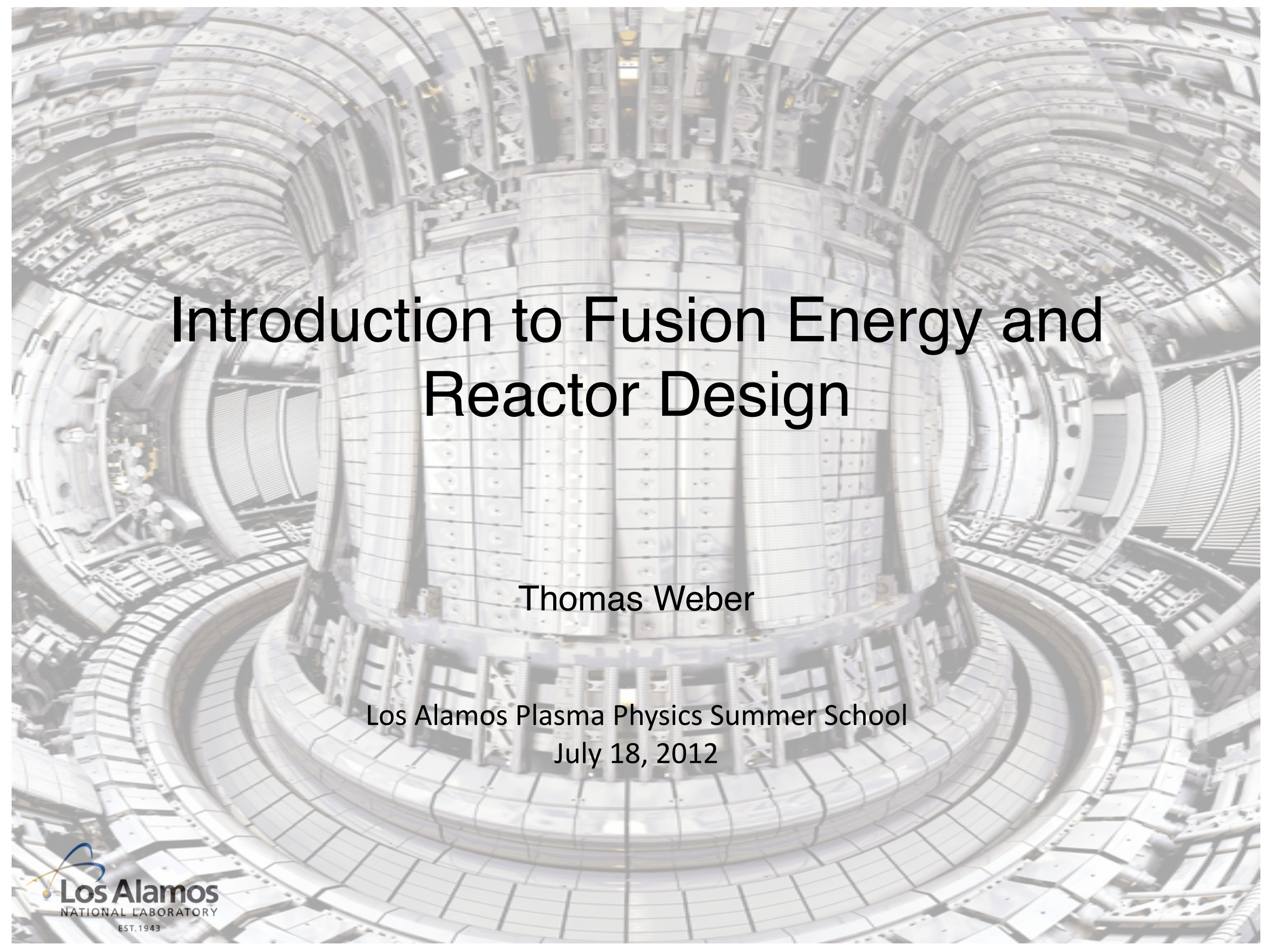




\section{Exothermic nuclear reactions}

Mass-energy equivalence:

$E=m c^{2}$

Binding energy of an atomic nucleus:

$B=\Delta m c^{2}$

$\Delta m=Z m_{p}+(A-Z) m_{n}-m$

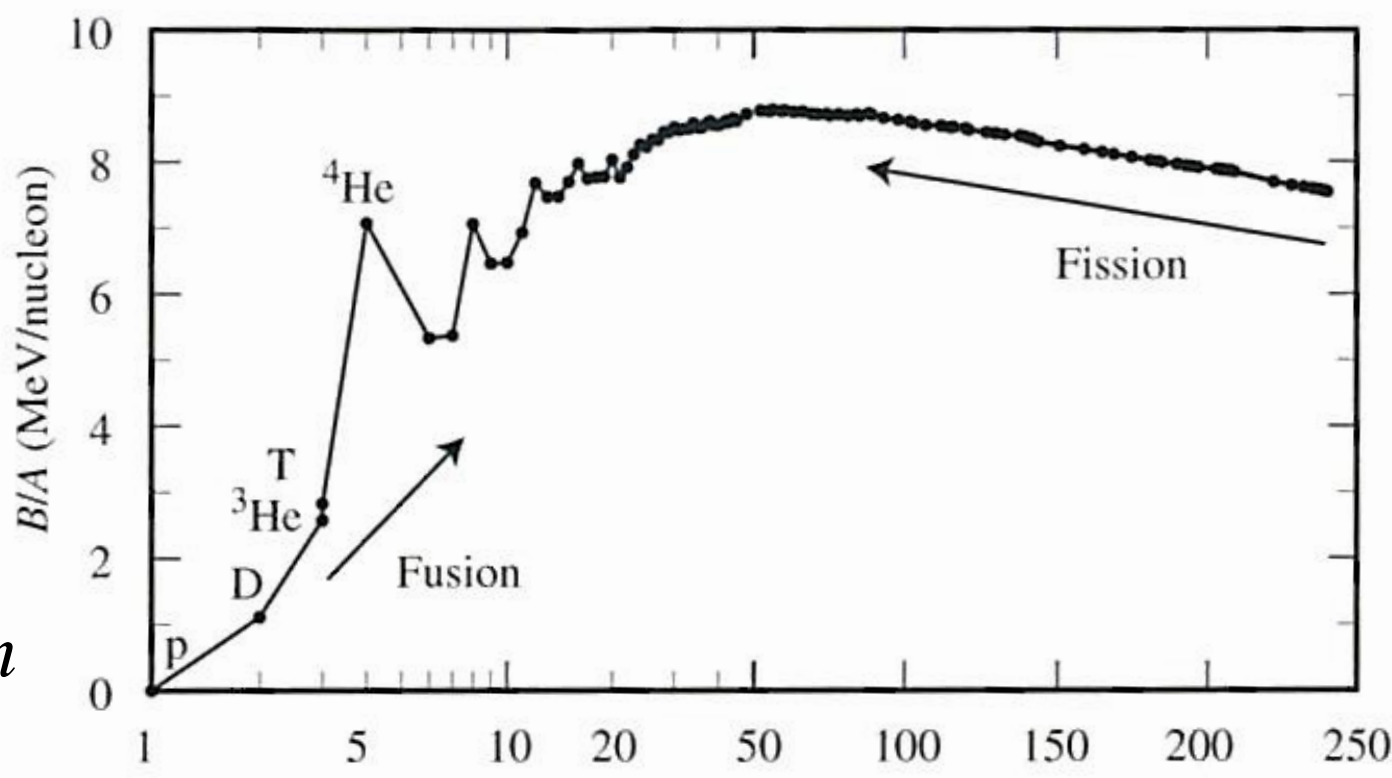

Energy released from initial to final state:

Mass number $A$

$Q=\sum_{f} B_{f}-\sum_{i} B_{i}$

Energy is released through fission or fusion of nuclei toward Iron ( $A \approx 56$, highest binding energy per nucleon). 


\section{Fusion}

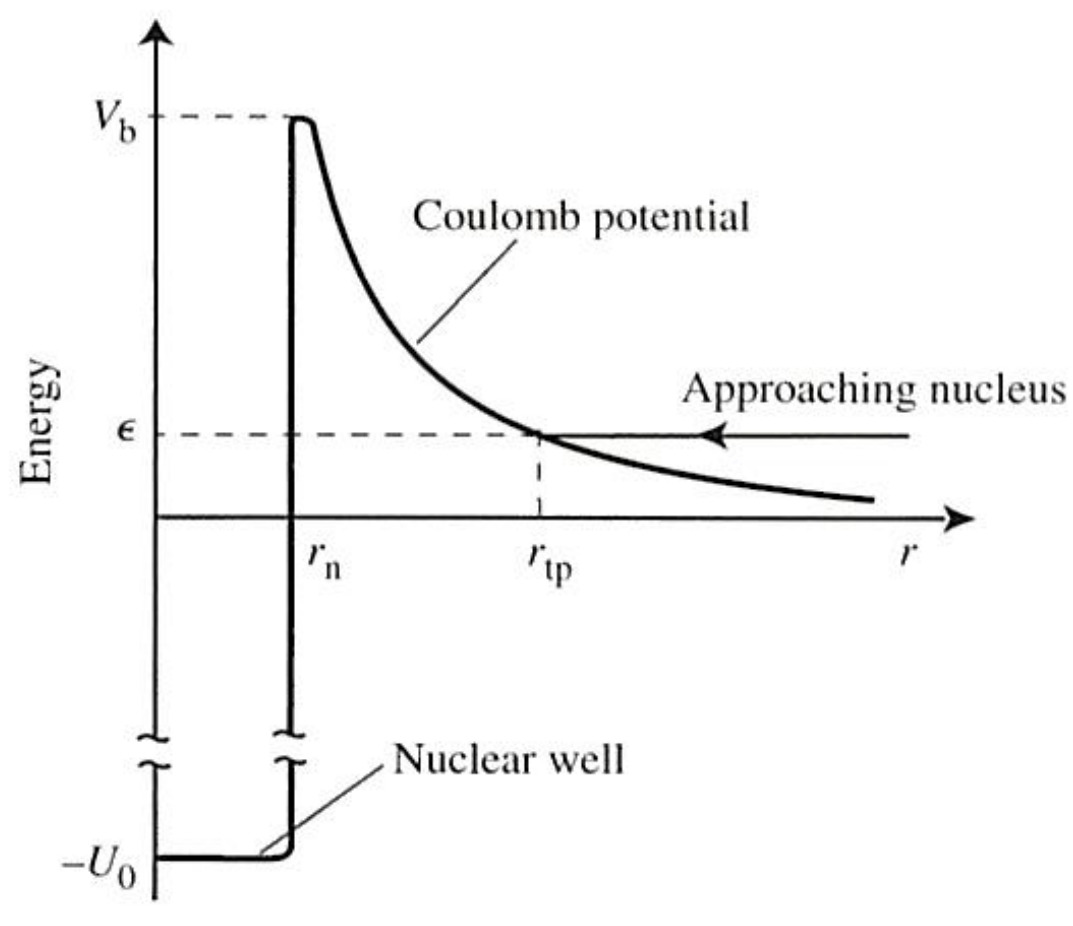

Classical Coulomb potential:

$$
V_{c}(r)=\frac{Z_{1} Z_{2} e^{2}}{r}
$$

Nuclear radius:

$r_{n} \approx 1.44 \times 10^{-13}\left(A_{1}^{1 / 3}+A_{2}^{1 / 3}\right)[\mathrm{cm}]$

$V_{c}$ is on the order of $1 \mathrm{MeV} ! ! !$

S. Atenzi, J. Meyer-ter-vehn, Int. Series of Monographs on Phys. - 125, 2004 


\section{Fusion Cross Section}

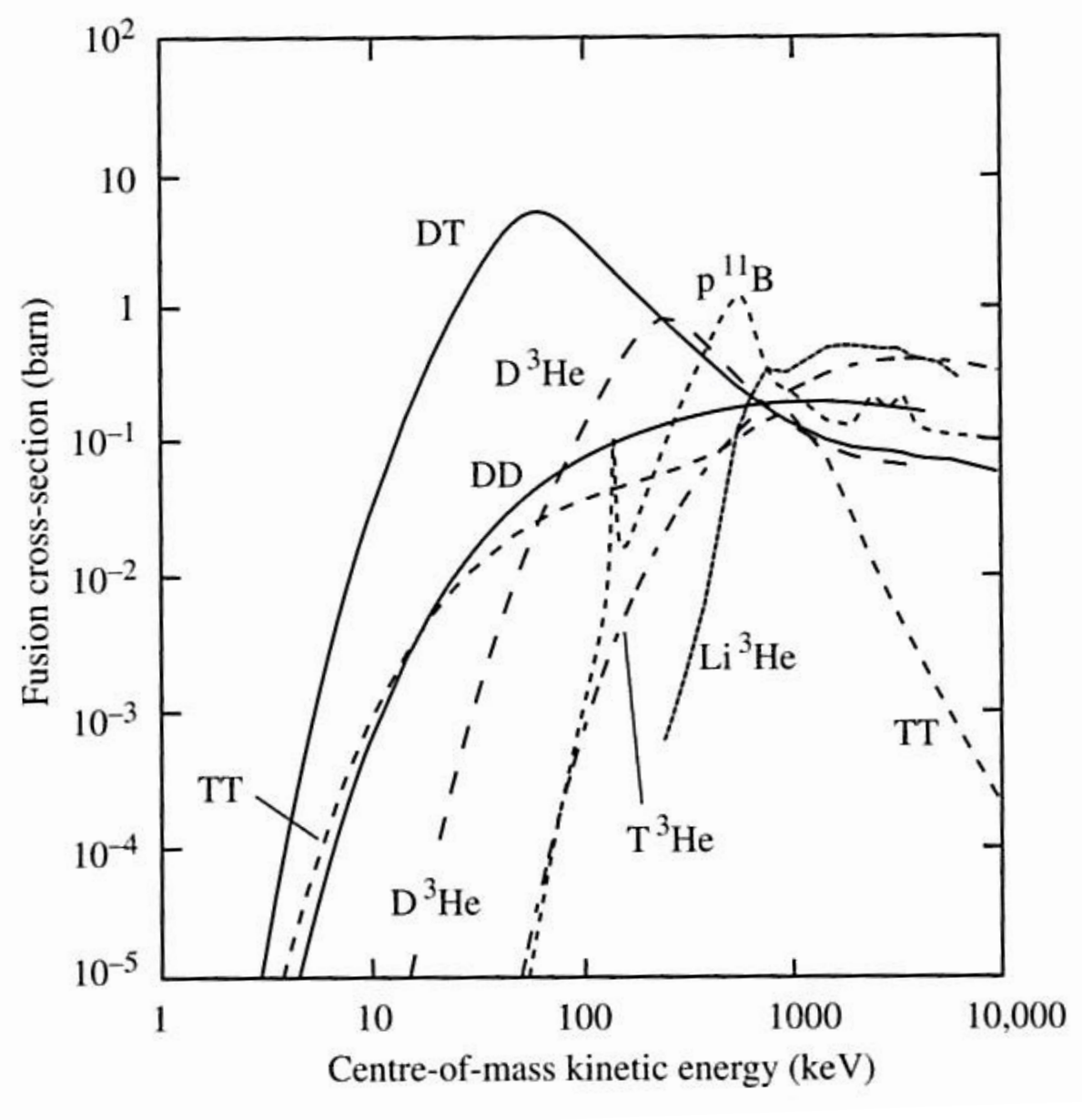

Fortunately, Quantum Mechanics comes to the rescue!

$$
\begin{aligned}
& \sigma \approx \sigma_{\text {geom. }} T_{\text {barrier }} R_{\text {prob }} . \\
& \sigma \approx \frac{\exp \left(-\sqrt{\varepsilon_{G} / \varepsilon}\right)}{\varepsilon} S(\varepsilon)
\end{aligned}
$$

$S(\varepsilon)$ is the astrophysical S factor.

S usually varies more weakly as function of energy than the Gamow factor.

DT reaction has relatively large $S(0)$ and relatively small Gamow energy. 


\section{Reactivity}

Volume swept out per unit time:

$$
v \sigma(v)
$$

Inverse time to intersection (reaction rate) of 1 atom traveling at speed $v$ through a target of density $n$ :

$$
n v \sigma(v)
$$

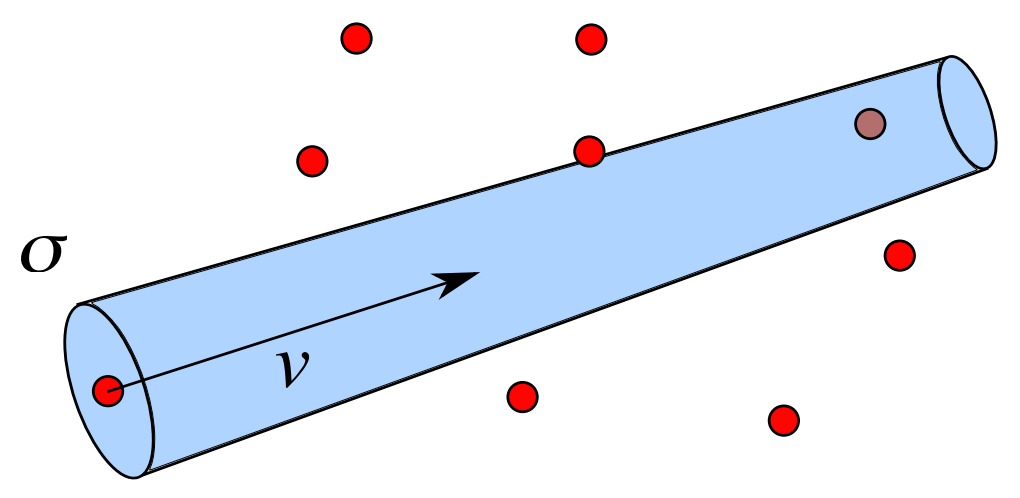

Reactivity: probability of reaction per unit time per unit density $=v \sigma$

Averaged Reactivity: average over target velocities $\langle\sigma v\rangle=\int_{0}^{\infty} \sigma(v) f(v) v d v$

Reaction Rate: number of reactions per unit time, per unit volume.

$$
R_{12}=\frac{n_{1} n_{2}}{1+\delta_{12}}\langle\sigma v\rangle=\frac{f_{1} f_{2}}{1+\delta_{12}} n^{2}\langle\sigma v\rangle
$$




\section{Maxwell averaged reactivity}

Average reactivity using two species:

$$
\begin{aligned}
& \langle\sigma v\rangle=\iint d \vec{v}_{1} d \vec{v}_{2} \sigma_{1,2}(v) v f_{1}\left(v_{1}\right) \\
& \text { where } \quad v=\left|\vec{v}_{1}-\vec{v}_{2}\right|
\end{aligned}
$$

Where $f_{i}$ is the Maxwell-Boltzmann distribution function (thermal equilibrium).

$$
f_{j}\left(v_{j}\right)=\left(\frac{m_{j}}{2 \pi k T}\right)^{3 / 2} \exp \left(\frac{-m_{j} v_{j}^{2}}{2 k T}\right)
$$

Substituting in for the distribution function and using center of mass velocity and reduced mass:

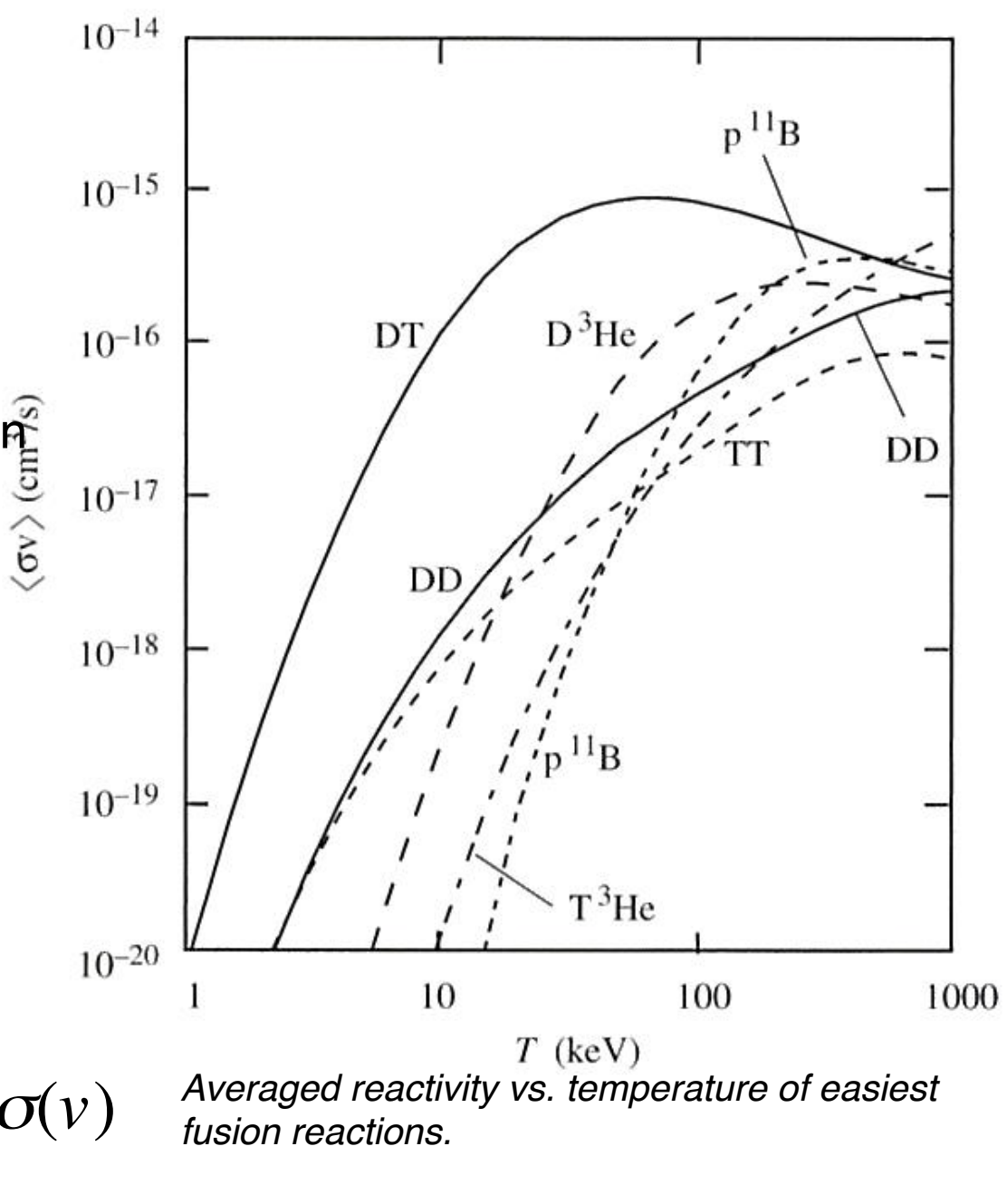

$\langle\sigma v\rangle=\left(\frac{m_{r}}{2 \pi k T}\right)^{3 / 2} \int d \vec{v} \exp \left(\frac{-m_{r}}{2 k T} \vec{v}^{2}\right) v \sigma(v)$ 


\section{p-p cycle}

Dominant cycle in our sun.

First stage of ${ }^{2} \mathrm{He}$ beta decaying into $\mathrm{D}$ is extremely rare. ${ }^{2} \mathrm{He}$ usually decays into $2 \mathrm{H}$.

Branches: different outcomes of a reaction.

4 paths from ${ }^{3} \mathrm{He}$ to ${ }^{4} \mathrm{He}$ :

- pp I- ${ }^{3} \mathrm{He}+{ }^{3} \mathrm{He}(86 \%$ in sun)

- pp II - ${ }^{3} \mathrm{He}+{ }^{4} \mathrm{He}(14 \%$ in sun)

- pp III $-{ }^{3} \mathrm{He}+{ }^{4} \mathrm{He}(0.11 \%$ in sun $)$

- $\mathrm{pp}$ IV $-{ }^{3} \mathrm{He}+\mathrm{p}$ (very rare in sun)

pp III cycle generates high energy

neutrinos and is very important to

the solar neutrino problem

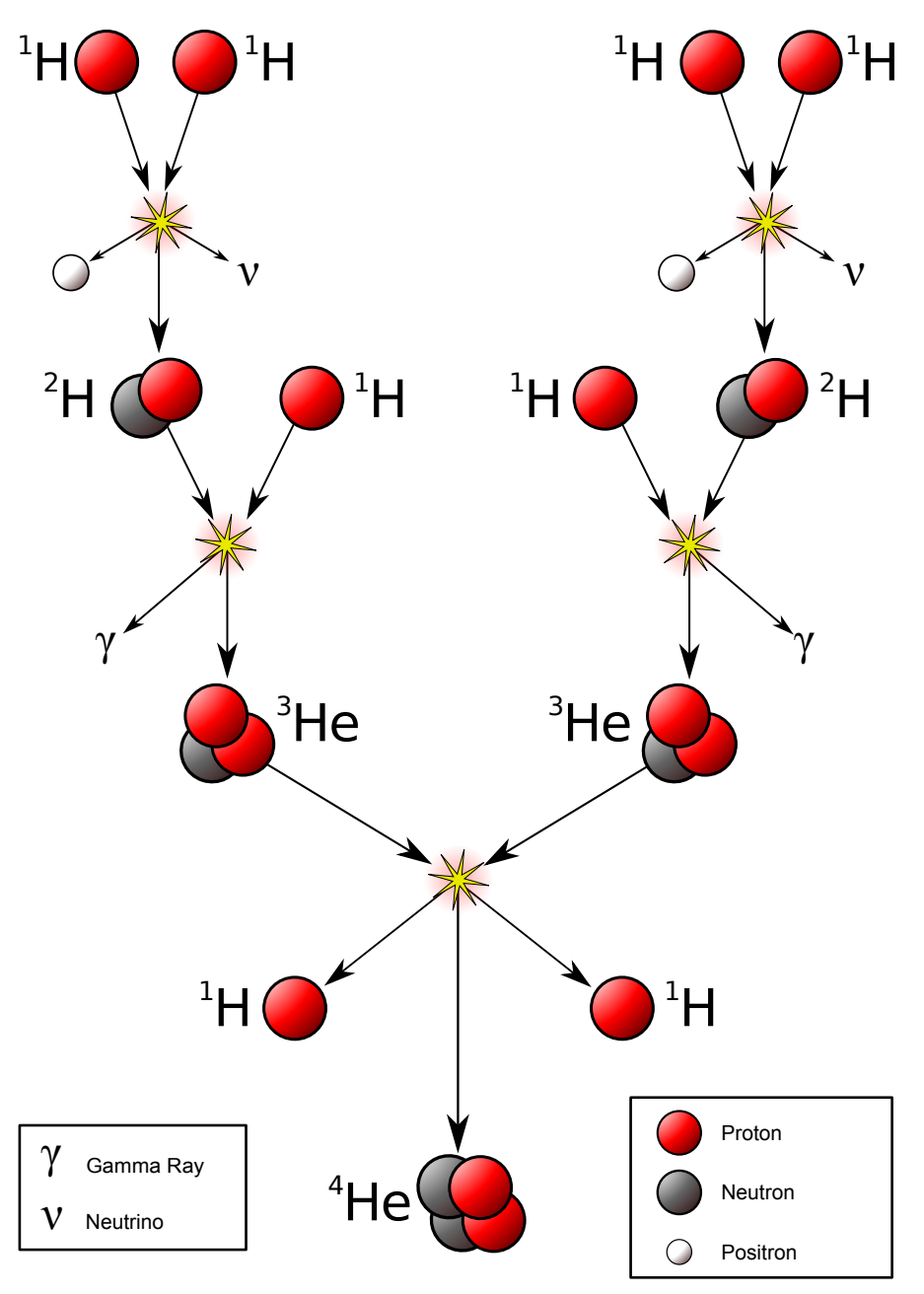

p-p cycle showing ppl branch (most probable) 


\section{CNO cycle}

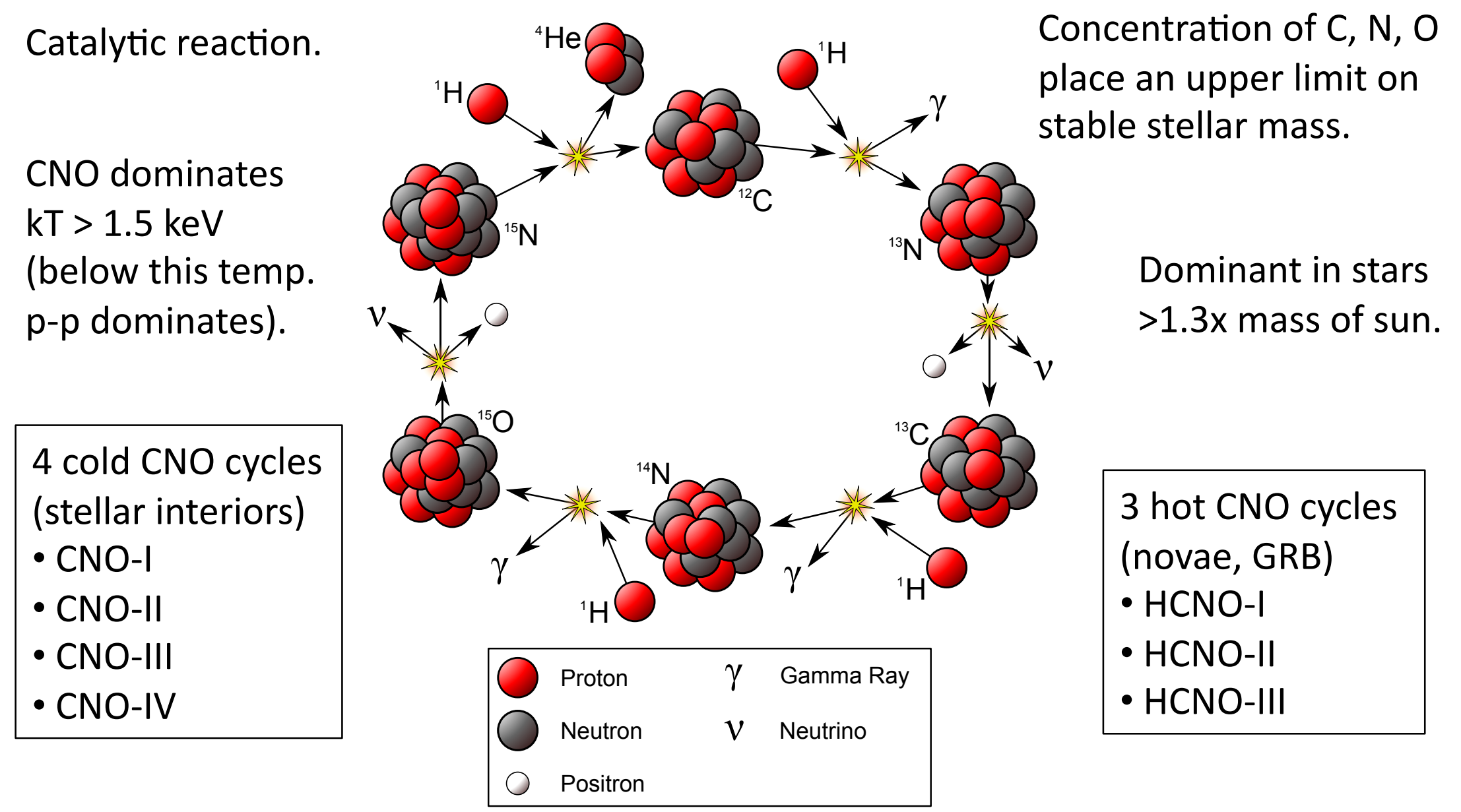

Los Alamos Plasma Physics Summer

School July 18, 2012 


\section{Cosmic nucleosynthesis}

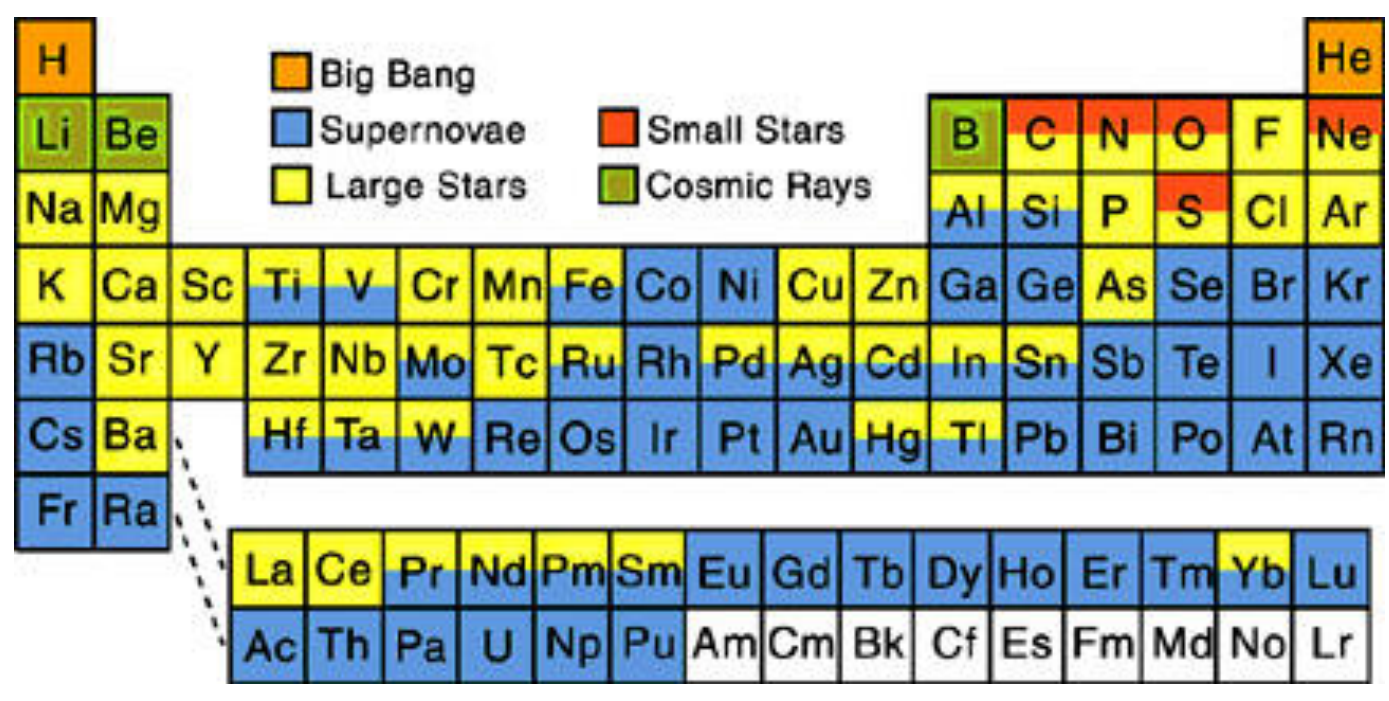

4 major sources:

- big bang

- stellar interiors

- supernovae

- cosmic ray spallation

Iron is lowest energy state (highest binding energy).

Higher energy species can be produced in exothermic reactions or in endothermic reaction in high-energy systems.

- supernovae

- cosmic rays

- particle accelerators

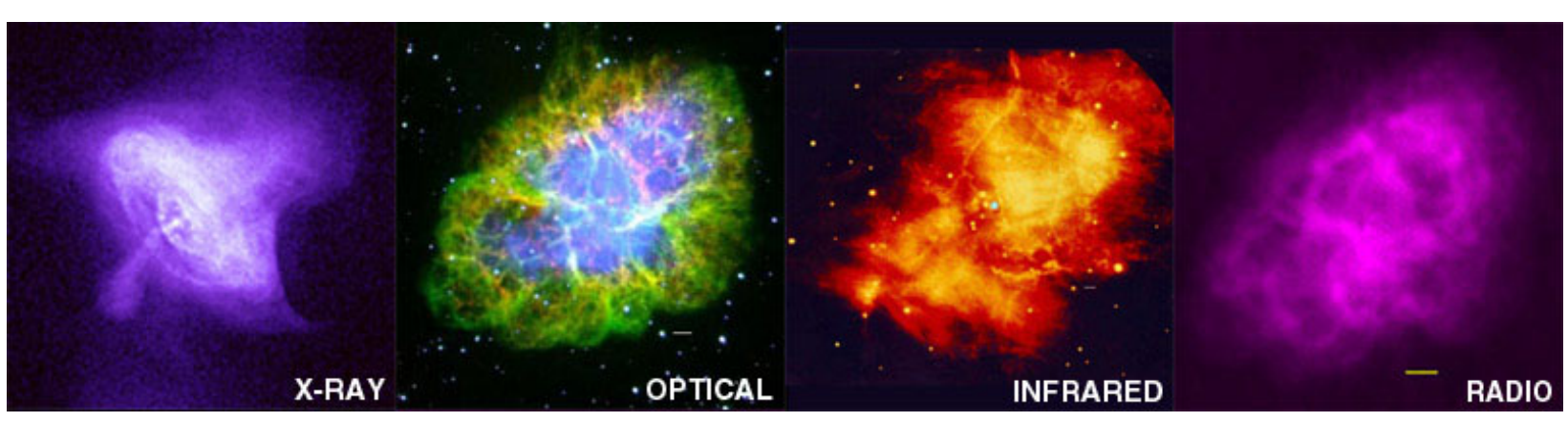

Los Alamos Plasma Physics Summer School July 18, 2012 


\section{Important terrestrial fusion reactions}

$$
\begin{aligned}
& \mathrm{D}+\mathrm{T} \rightarrow{ }^{3} \mathrm{He}+\mathrm{n} \\
& \mathrm{D}+\mathrm{D} \rightarrow\left\{\begin{array}{l}
\mathrm{T}+\mathrm{p} \\
{ }^{3} \mathrm{He}+\mathrm{n} \\
\alpha+\gamma
\end{array}\right. \\
& \mathrm{T}+\mathrm{T} \rightarrow \alpha+2 \mathrm{n} \\
& \mathrm{D}+{ }^{3} \mathrm{He} \rightarrow \alpha+\mathrm{p} \\
& { }^{3} \mathrm{He}+{ }^{3} \mathrm{He} \rightarrow \alpha+2 \mathrm{p} \\
& \mathrm{p}+{ }^{11} \mathrm{~B} \rightarrow 3 \alpha
\end{aligned}
$$




\section{Rare isotopes}

Deuterium: stable isotope of Hydrogen

- mass = 2 amu

- naturally occurs at $0.03 \%$

- can be harvested from ocean water

- constituent of "heavy water"
Helium-3: aka. Tralphium (stable)

- mass $=3$ amu.

- naturally occurs at $0.000137 \%$ (earth)

- higher abundance on moon (1-50 bbp)

- proposed moon mining once fusion is

"solved"

Tritium: radioactive isotope of Hydrogen

- mass $=3$ amu

- half-life $=12.32$ years.

- extremely rare (essentially man-made)

- very toxic

- Produced in fission, decay, high energy collisions. 


\section{Lawson Criteria: considering losses}

Conditions required for a fusion system to reach "ignition" (Lawson 1955).

Ignition: plasma self heating from

fusion products $>$ loss rate.

Reaction rate from earlier:

$R_{D T}=n_{D} n_{T}\langle\sigma v\rangle=\frac{1}{4} n_{e}^{2}\langle\sigma v\rangle$

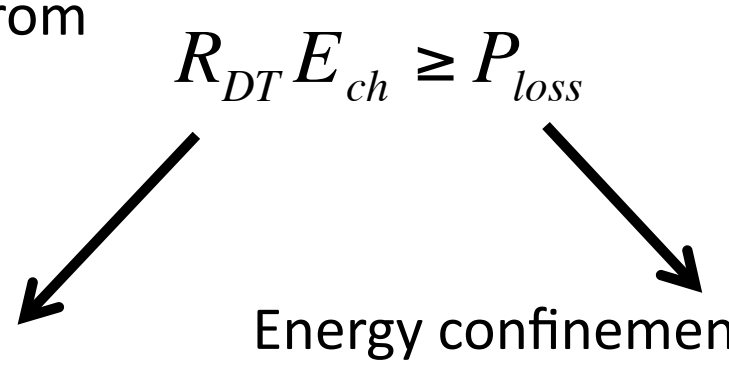

Energy confinement time: $\tau_{E}=\frac{W}{P_{\text {loss }}}$

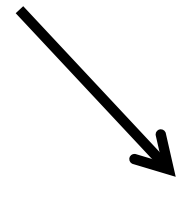

where $W=3 n_{e} k T$

$$
\frac{1}{4} n_{e}^{2}\langle\sigma v\rangle E_{c h} \geq \frac{3 n_{e} k t}{\tau_{E}}
$$

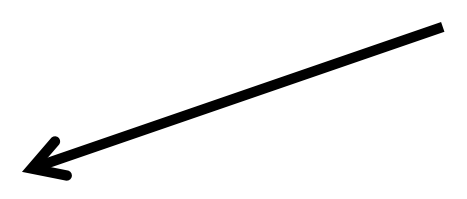

$$
\text { Lawson Criteria: } \quad n_{e} \tau_{E} \geq \frac{12 k T}{E_{c h}\langle\sigma v\rangle}
$$




\section{Inserting values}

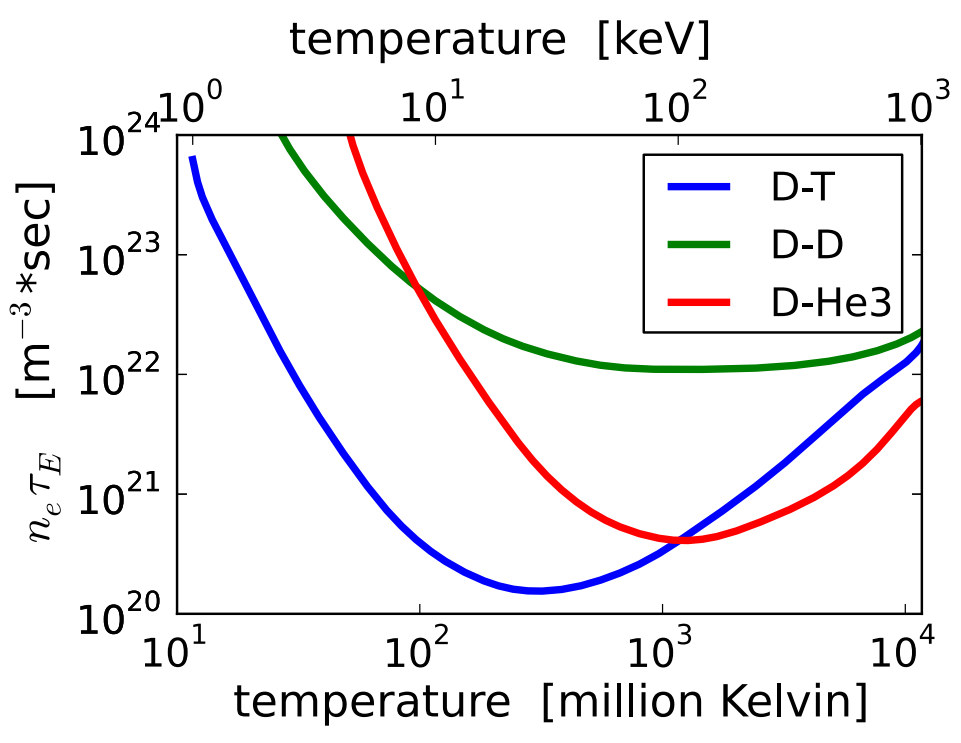

Required value of $n_{e} \tau_{E}$ depends on temperature.

Minimum for DT:

$$
n_{e} \tau_{E} \geq 1.5 \times 10^{20}\left[\mathrm{~s} / \mathrm{m}^{3}\right]
$$

temperature $[\mathrm{keV}]$

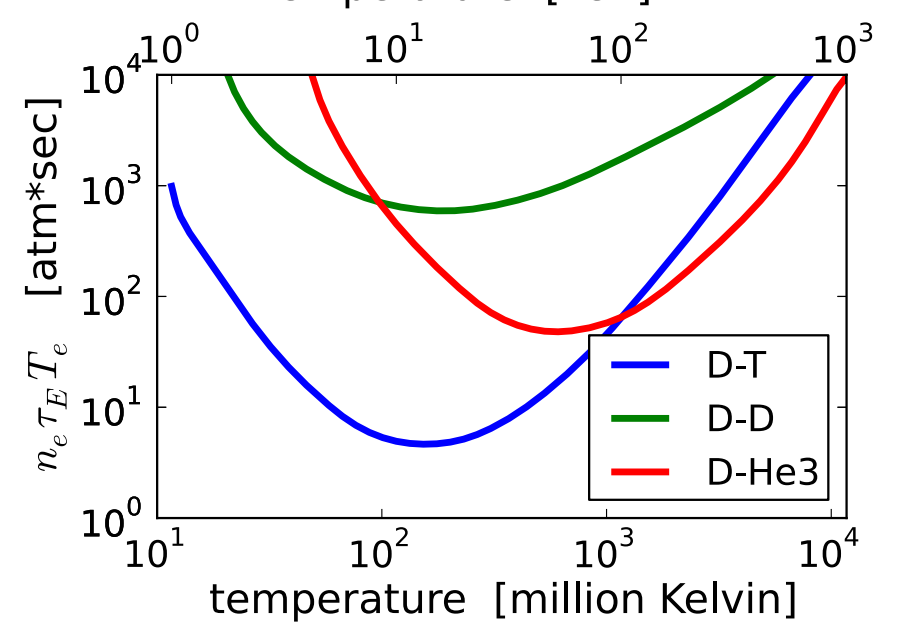

"Triple product" is typically a more useful figure of merit.

Minimum for DT:

$$
n_{e} \tau_{E} T \geq 10^{21}\left[\mathrm{keV} / \mathrm{m}^{3}\right]
$$




\section{Energy Gain Factor (Q)}

Ratio of fusion energy production to heating energy required.

$Q=\frac{P_{\text {fusion }}}{P_{\text {heatng }}}=\left(\eta_{\text {heat }} f_{\text {recir. } .} \eta_{\text {elect. }}\left(1-f_{\text {ch }}\right)\right)^{-1}$

where $\quad P_{\text {heatng }}=\eta_{\text {heat }} f_{\text {recirc. }} \eta_{\text {elect. }}\left(1-f_{\text {ch }}\right) P_{\text {fusion }}$

$\mathrm{Q}=1$ referred to as "breakeven".

$f_{c h}=0.2$ for DT

Assuming

$f_{\text {recirc. }}=0.2$

$\eta_{\text {heat }}=0.7$

$\eta_{\text {elect. }}=0.7$

Ignition corresponds to $Q=\infty$

(ignition is not a requirement for a practical, energy producing reactor) 


\section{Confinement concepts}
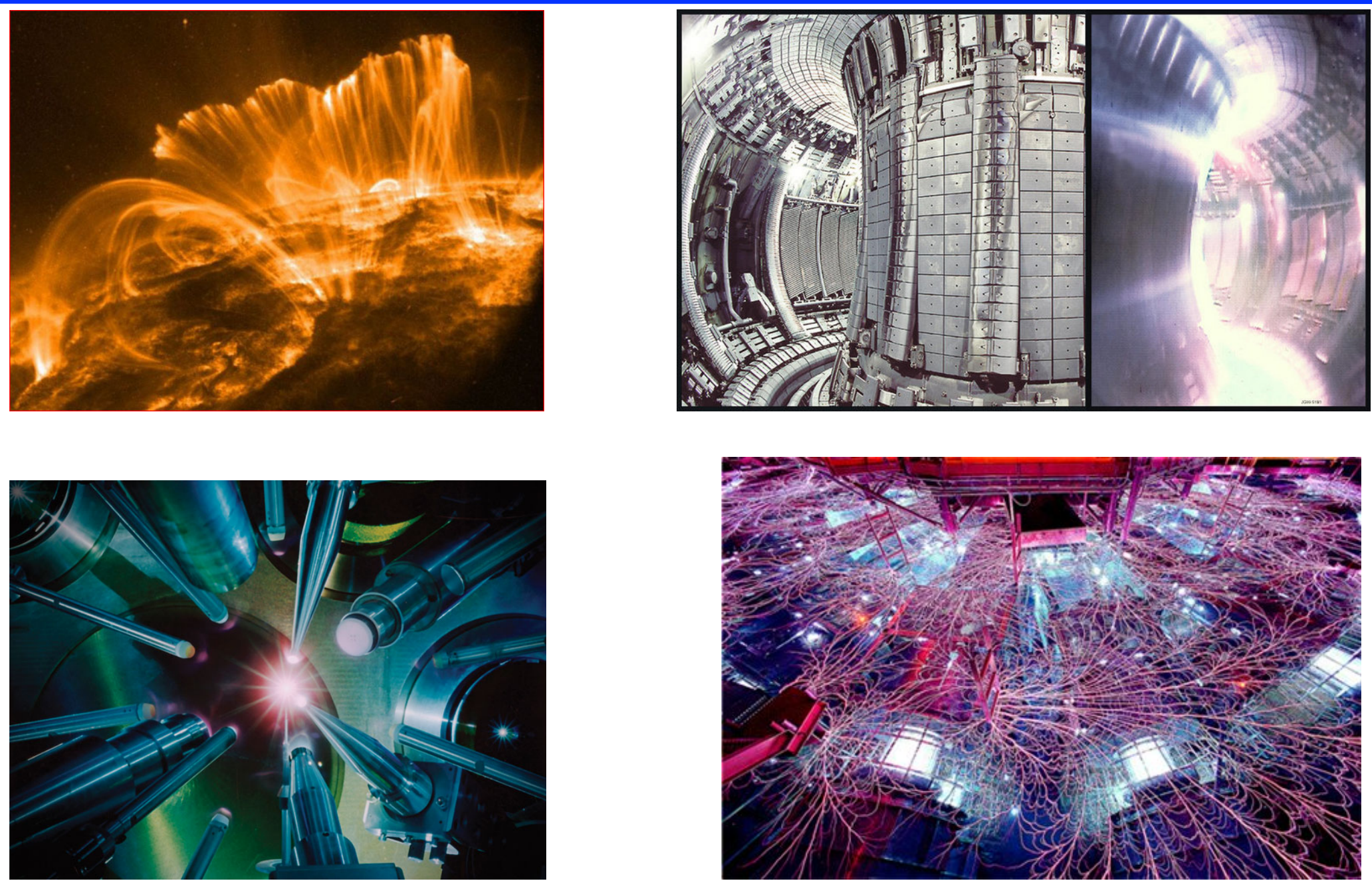


\section{Gravitational confinement}

- Exceptional confinement (billions of years)

- Capable of using common isotopes ( $p$-p cycle)

- Naturally occurring (we don't have to do any work!)

- Poor scaling...

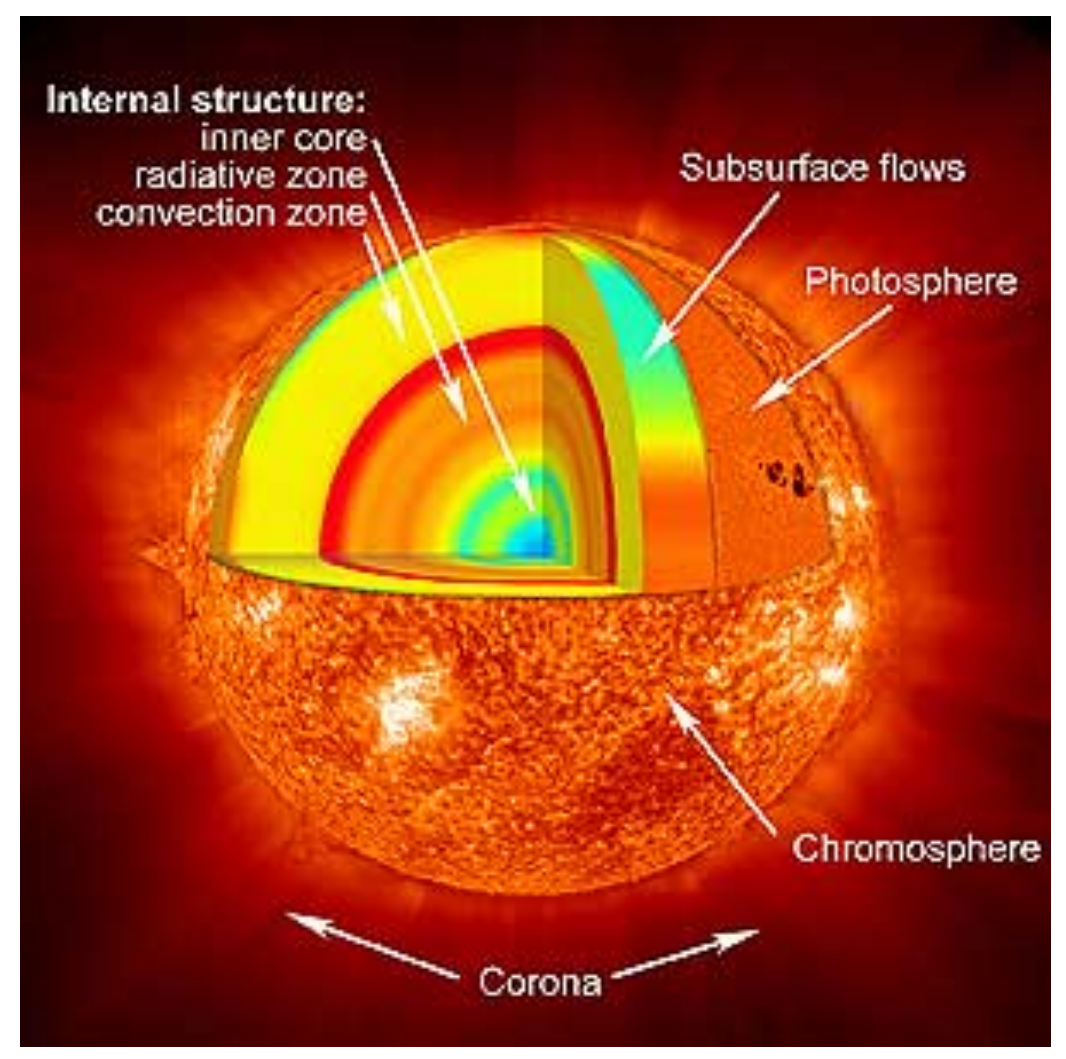




\section{Magnetic confinement Fusion Energy (MFE)}

- Use magnetic fields to confine the hot plasma.

- Hopefully will have better scaling than gravitational confinement fusion.

- Typically devices aimed at long-pulse or eventual steady-state operation.

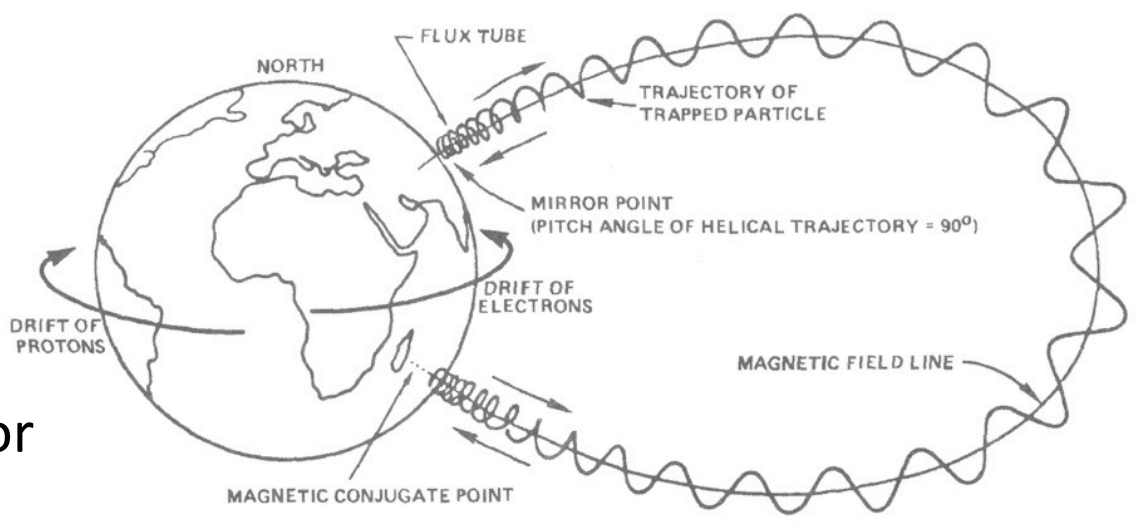

- Pressure ranges from near vacuum to material strength limits ( $<1 \mathrm{kbar}$ ). 


\section{z-pinch}
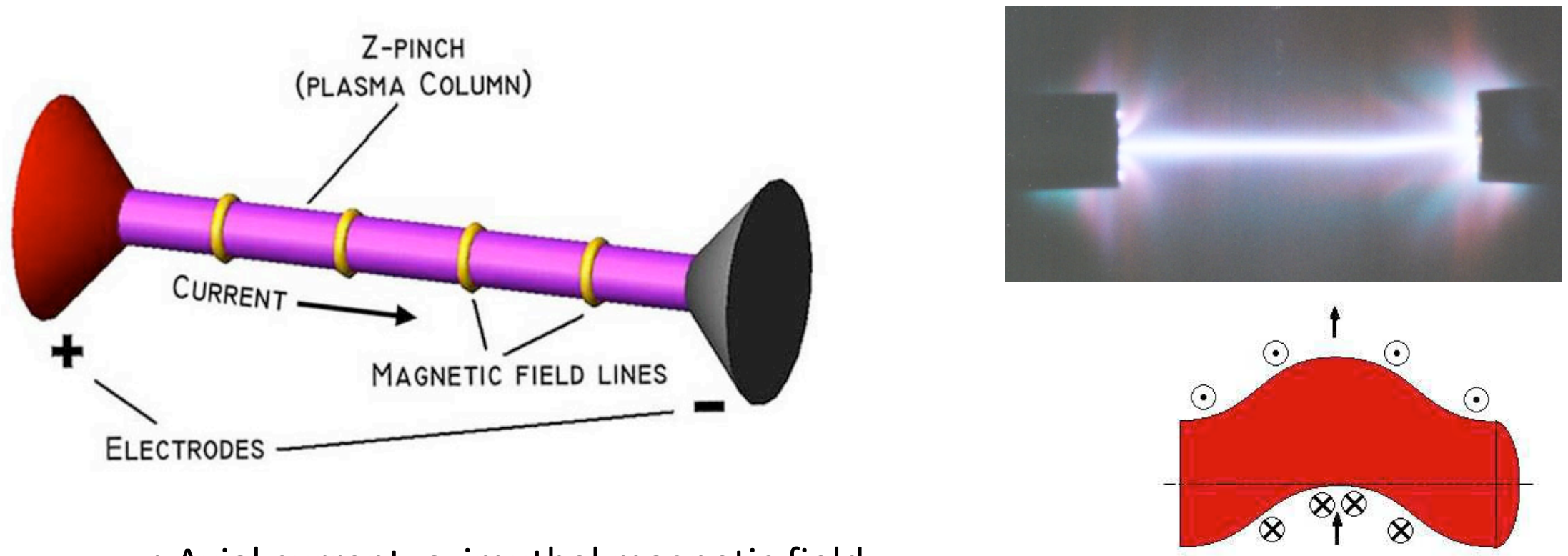

- Axial current, azimuthal magnetic field

- Material electrodes

- Pressure balance

- Notable variations:

- plasma focus

- cylindrical liner

-wire array

kink instability $(m=1)$

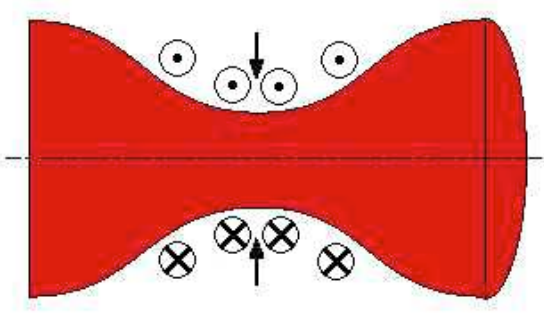

sausage instability $(m=0)$ 


\section{$\theta$-pinch}
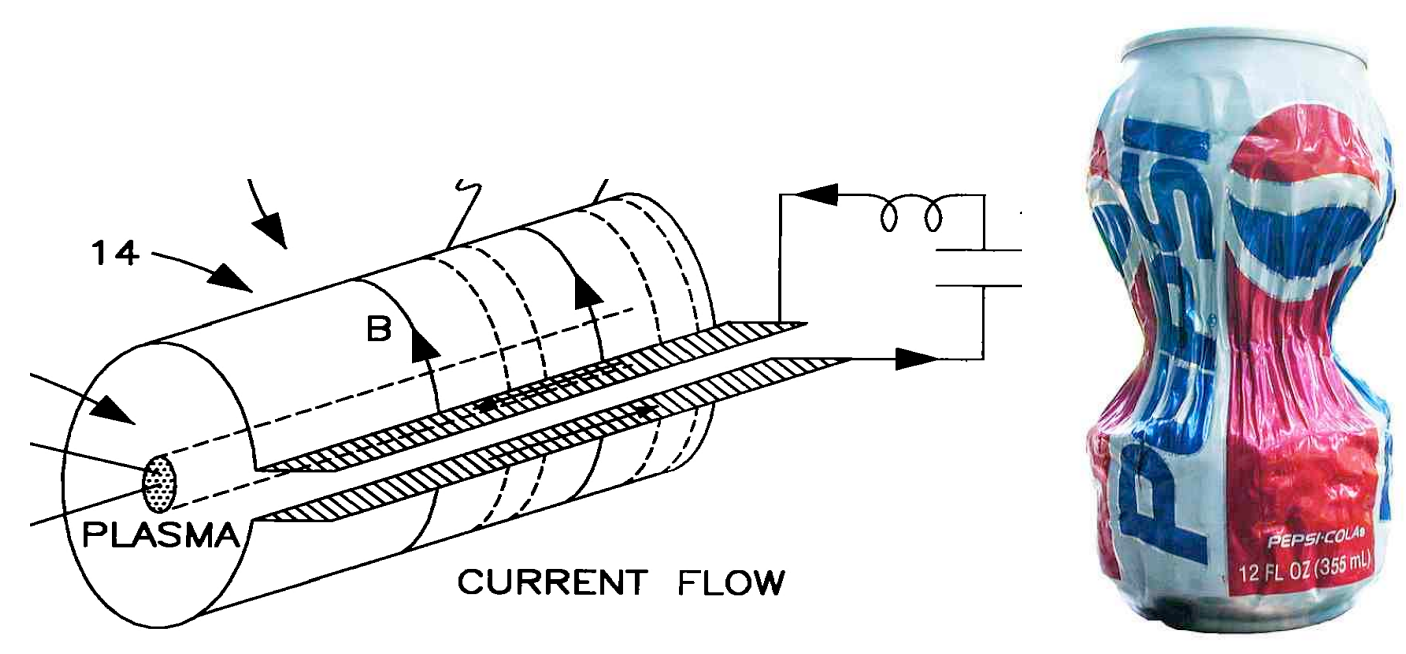

- Azimuthal current, axial magnetic field

- Magnetic coil driven

- Pressure balance

- Notable variations:

- Screw pinch: combination $\theta$ and $z$ pinchs

- another way to crush a can or liner

- possible way to form compact toroid 


\section{Tokamak}

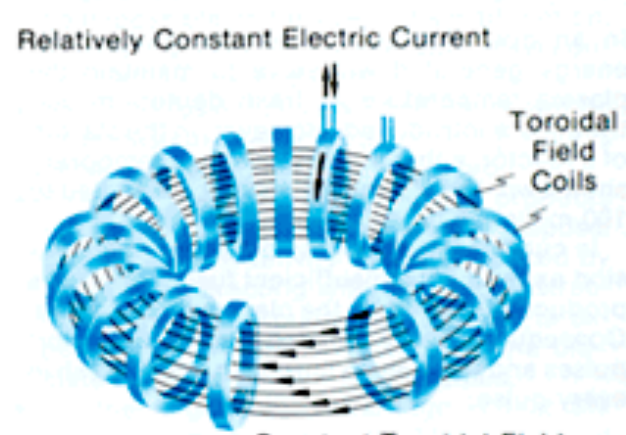

Constant Toroidal Field

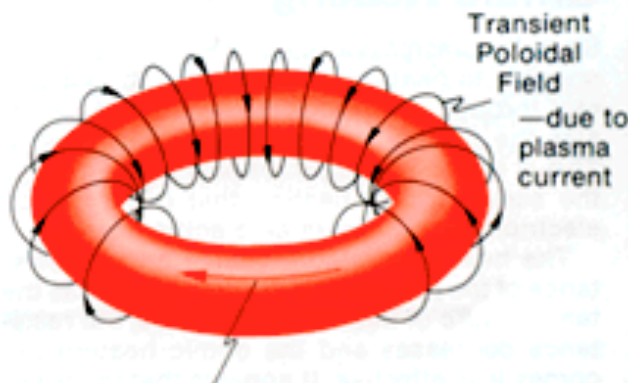

Transient Plasma Current

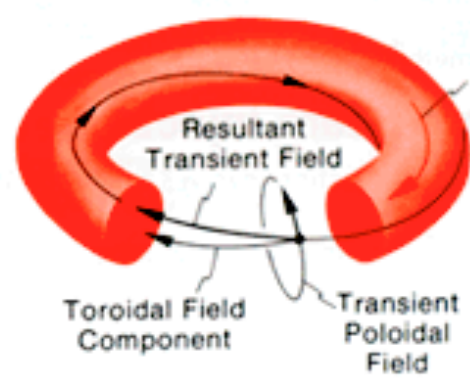

- Los Alamos

NATIONAL LABORATORY

EST. 1943

Plasma Current
- Russian acronym for "toroidal chamber with magnetic coils".

- Began in US as "perhapsatron"

- Primarily toroidal (axial) field with smaller poloidal (azimuthal) field.

- Needs current drive (goal of steady-state).

- Needs additional plasma heating.

- Confinement and instabilities.

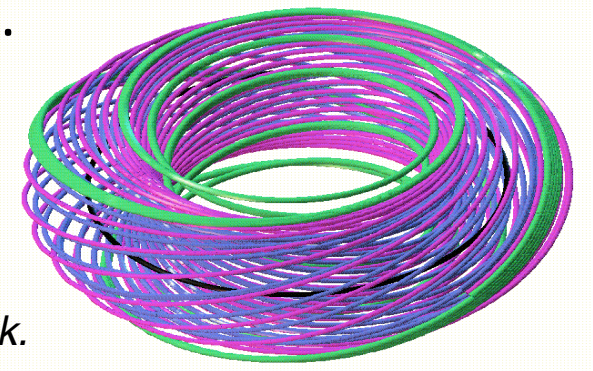




\section{Currently the leading MFE concept.}

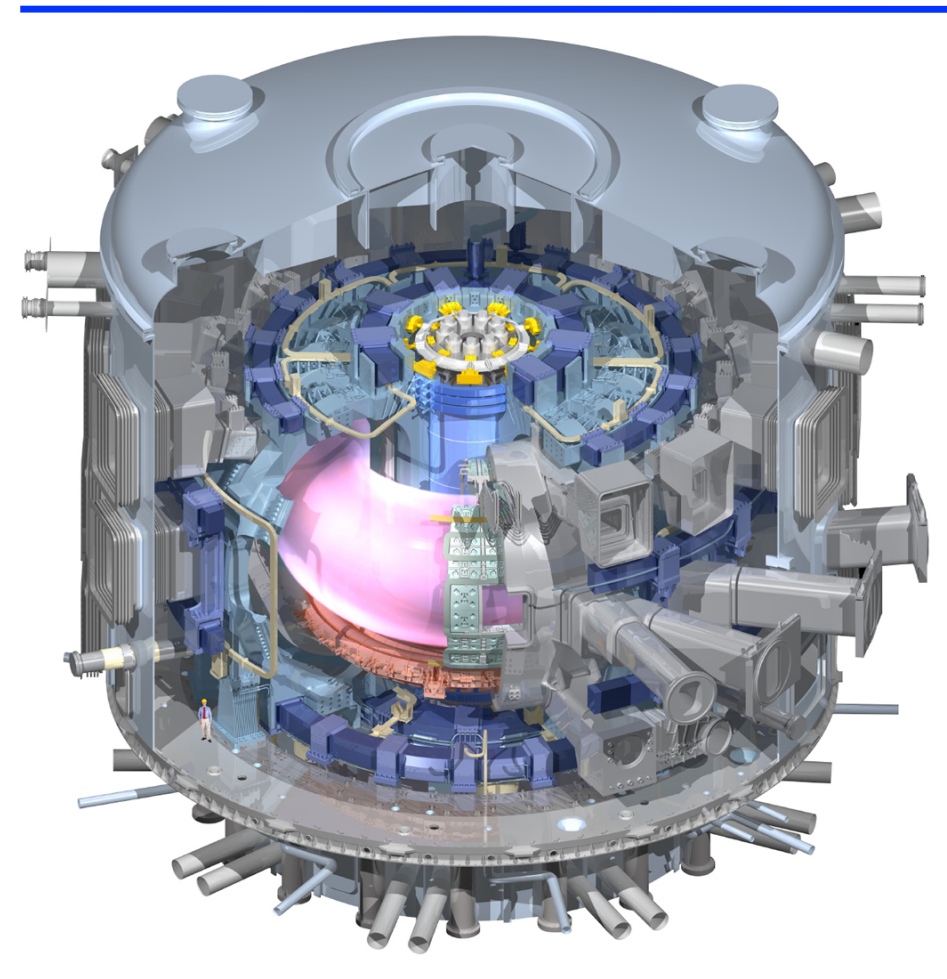

ITER schematic, Cadarasche France

ITER participants: United States, European Union, India, Japan, China, S. Korea, (previously Canada).

- Currently the leading MFE concept.

- Several large tokamaks around the world.

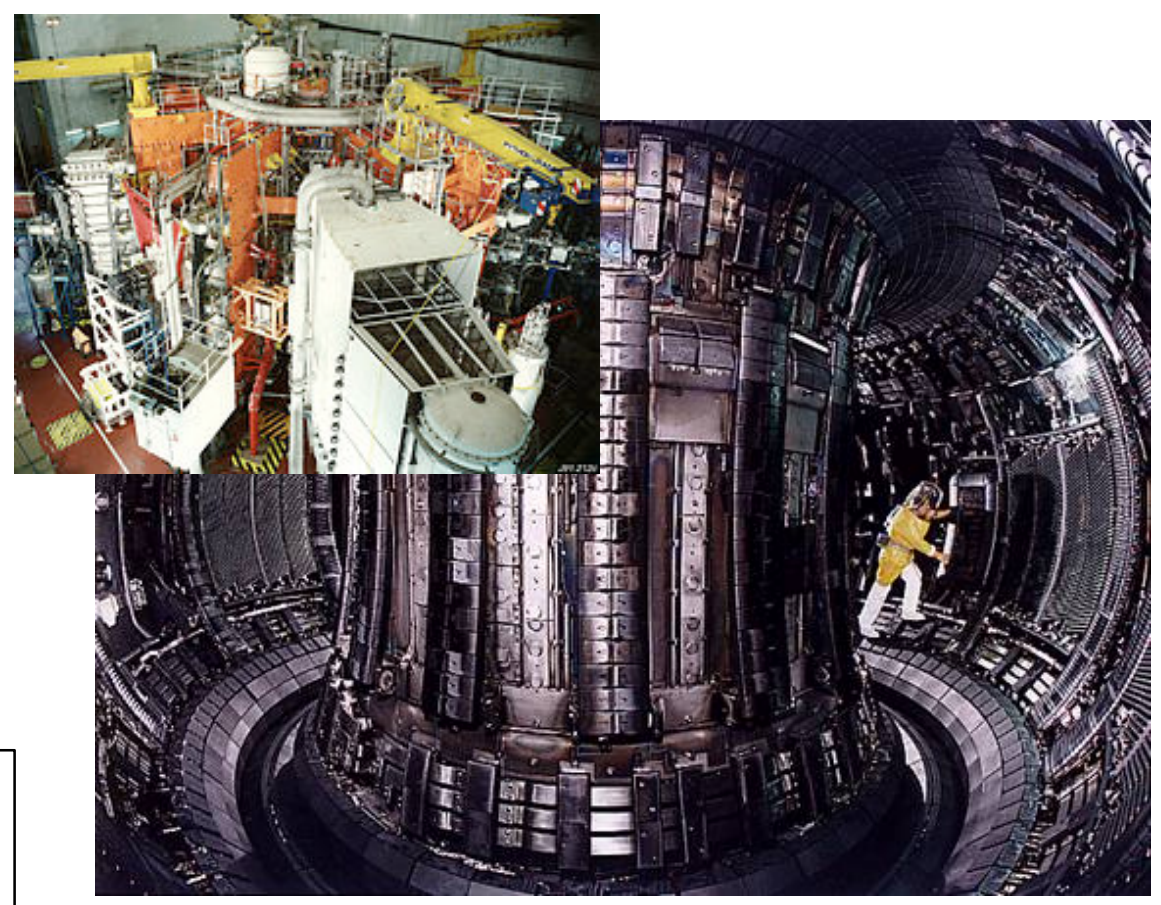

JET (Joint European Torus), Culham, Oxfordshire, UK. 


\section{Stellarator}

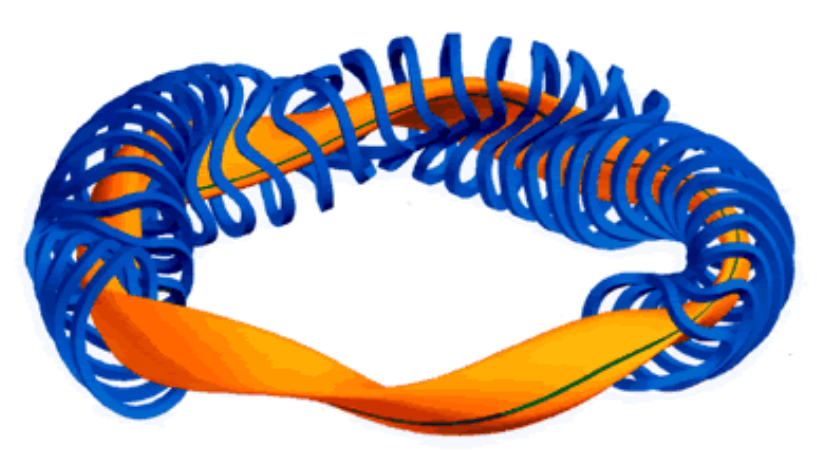

- US initial concept (superseded by Tokamak)

- Helical symmetry

- steady state

- complex magnetic coils

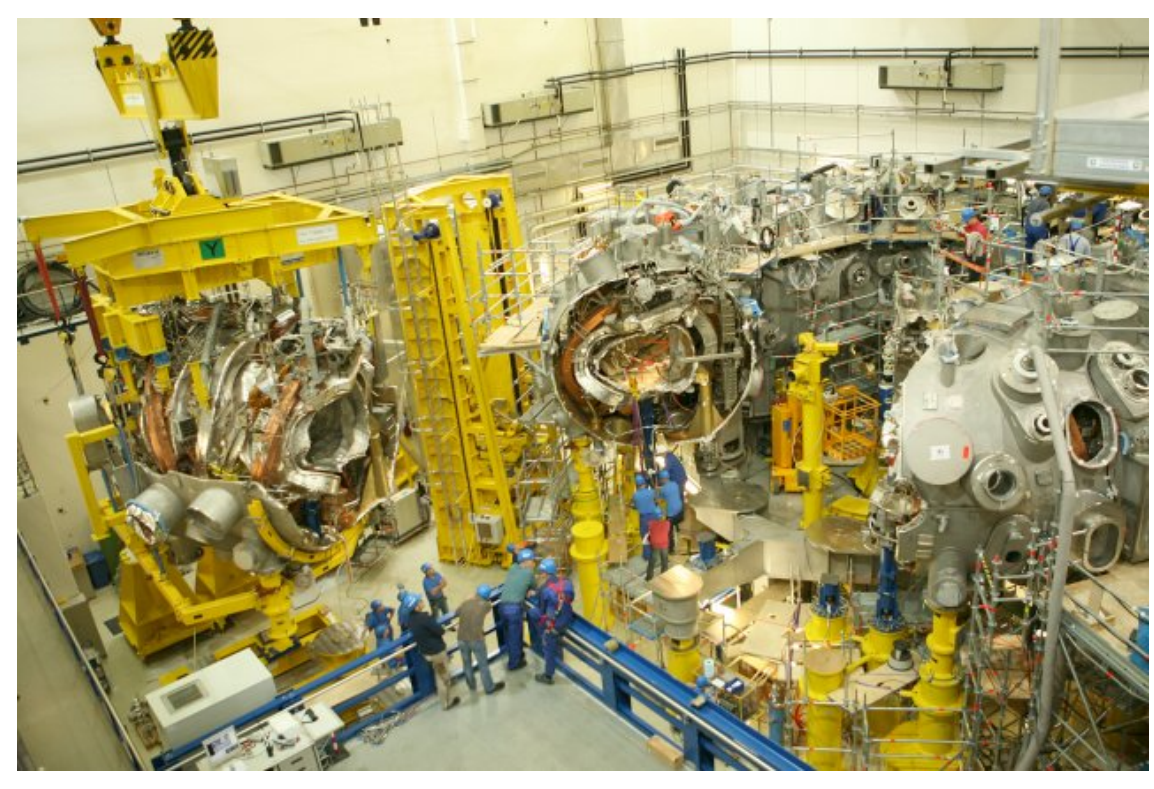

- lower energy density than Tokamak

W7X stellarator Greifswald, Germany

- Research producing continuously more advanced configurations. 


\section{Mirror}
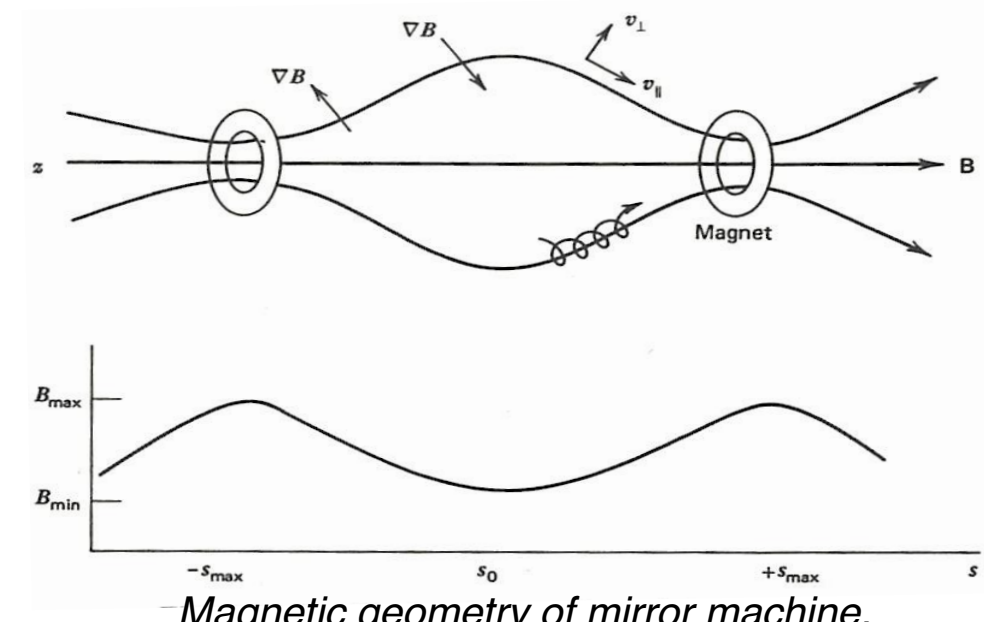

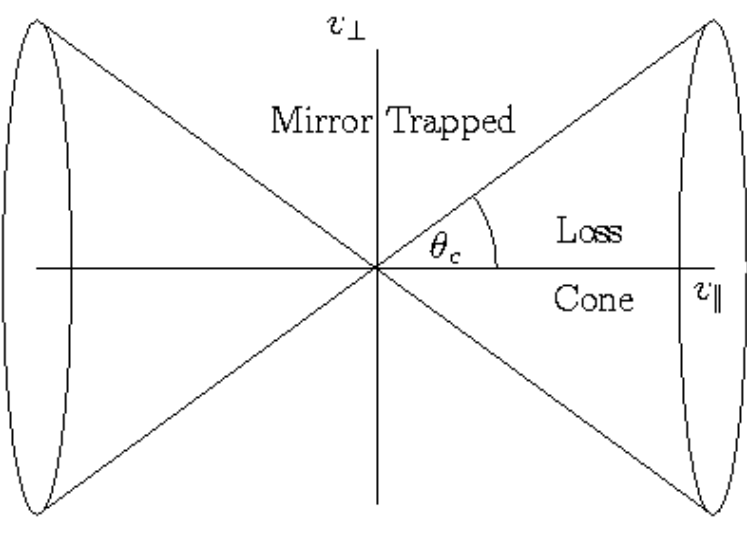

Loss cone

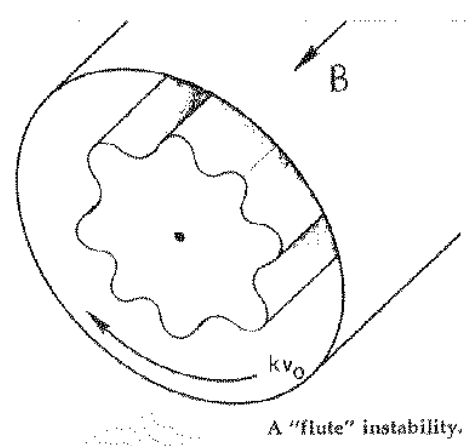

Flute instability

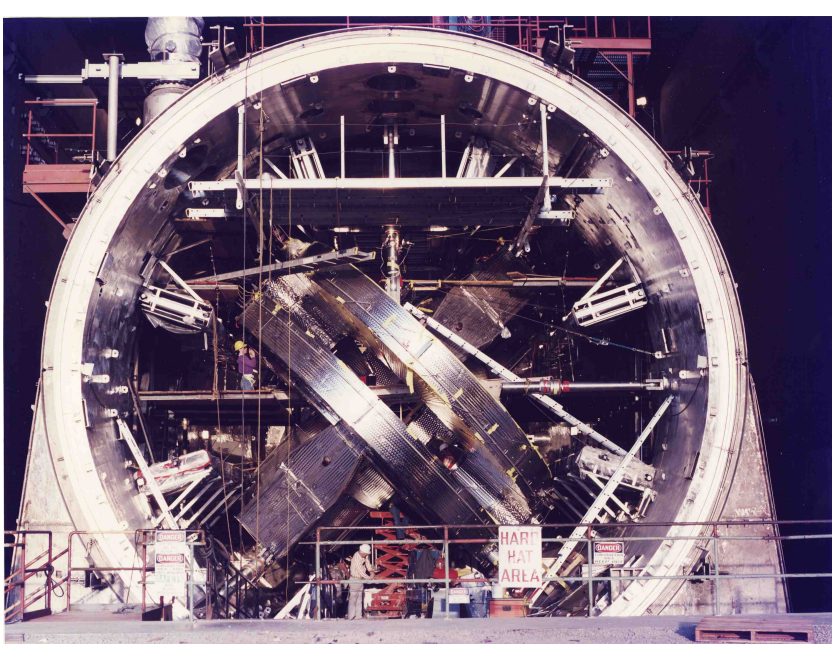

MFTF (mirror fusion test facility, Livermore CA 1977-1986

- Axial confinement via adiabatic invariant.

- Radial confinement due to reduced cross field transport.

- Heating via external mechanisms.

- End losses and instabilities an issue. 


\section{Others}

- Elmo bumpy torus

- Compact tokamak

- Spherical tokamak

- Compact torous: FRC, spheromak

- Reversed field pinch 


\section{Inertial Electrostatic Confinement (IEC)}

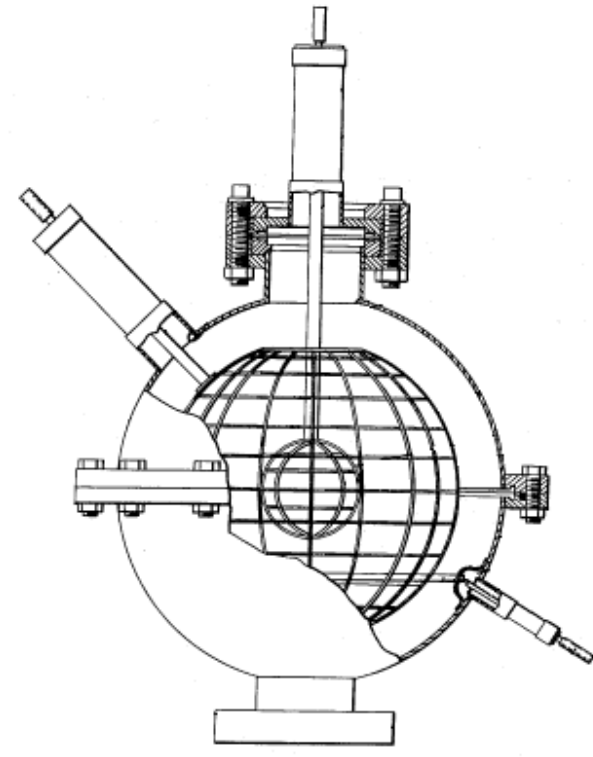

Hirsch-Meeks fusor From US patent $3,530,497$
Farnsworth-Hirsch fusor Polywell

-Space-charge limitation, bremsstralung, collision with electrodes removes energy, adds impurities.

-Beam instabilities quickly thermalize non-maxwellian distributions.

-lons in tail escape potential well before fusing.
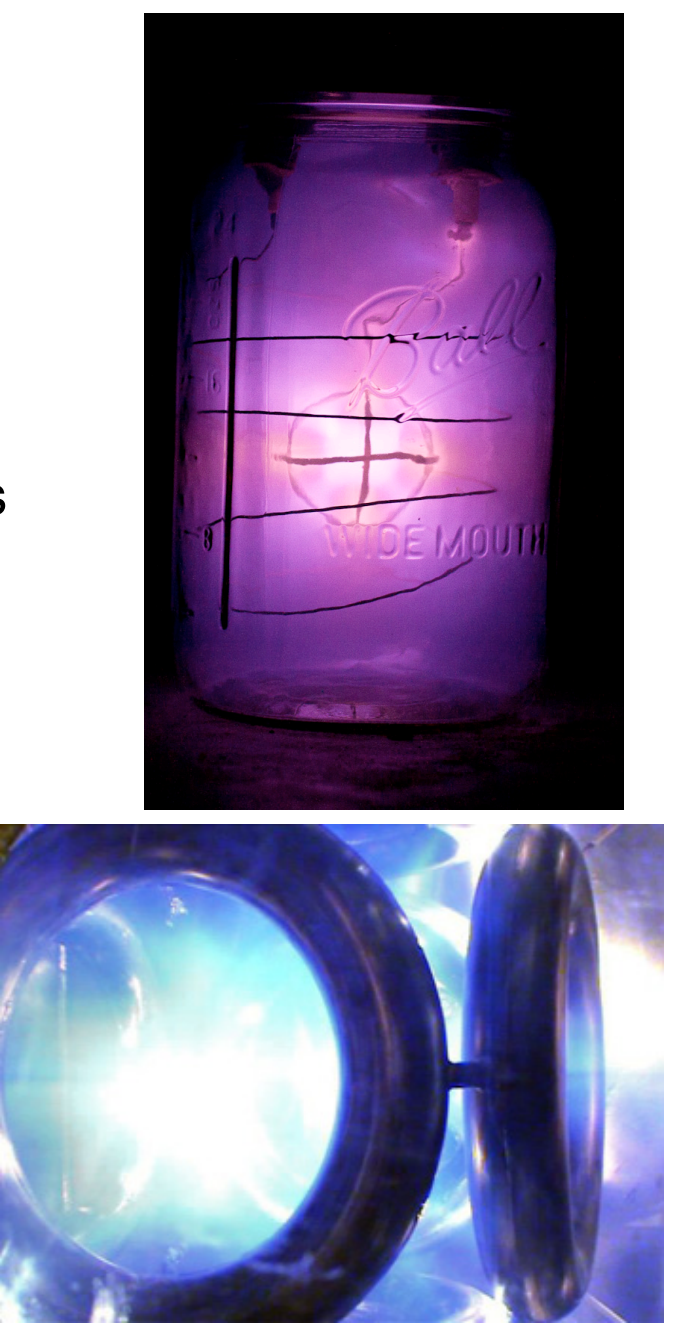


\section{Areal density}

Lawson criteria expressed in a different form for inertial confinement fusion.

Energy confinement time $\left(\tau_{\mathrm{E}}\right)$ in MFE systems is difficult to predict.

IFE energy confinement time is on the order of the radius over the sound speed

$$
\begin{gathered}
\tau_{E} \approx R / \sqrt{k T / m_{i}} \\
n \tau_{E} \approx n R / \sqrt{k T / m_{i}}
\end{gathered}
$$

Leads to $\rho$ R requirement for inertial confinement fusion schemes. 


\section{Inertial Confinement Fusion (ICF)}
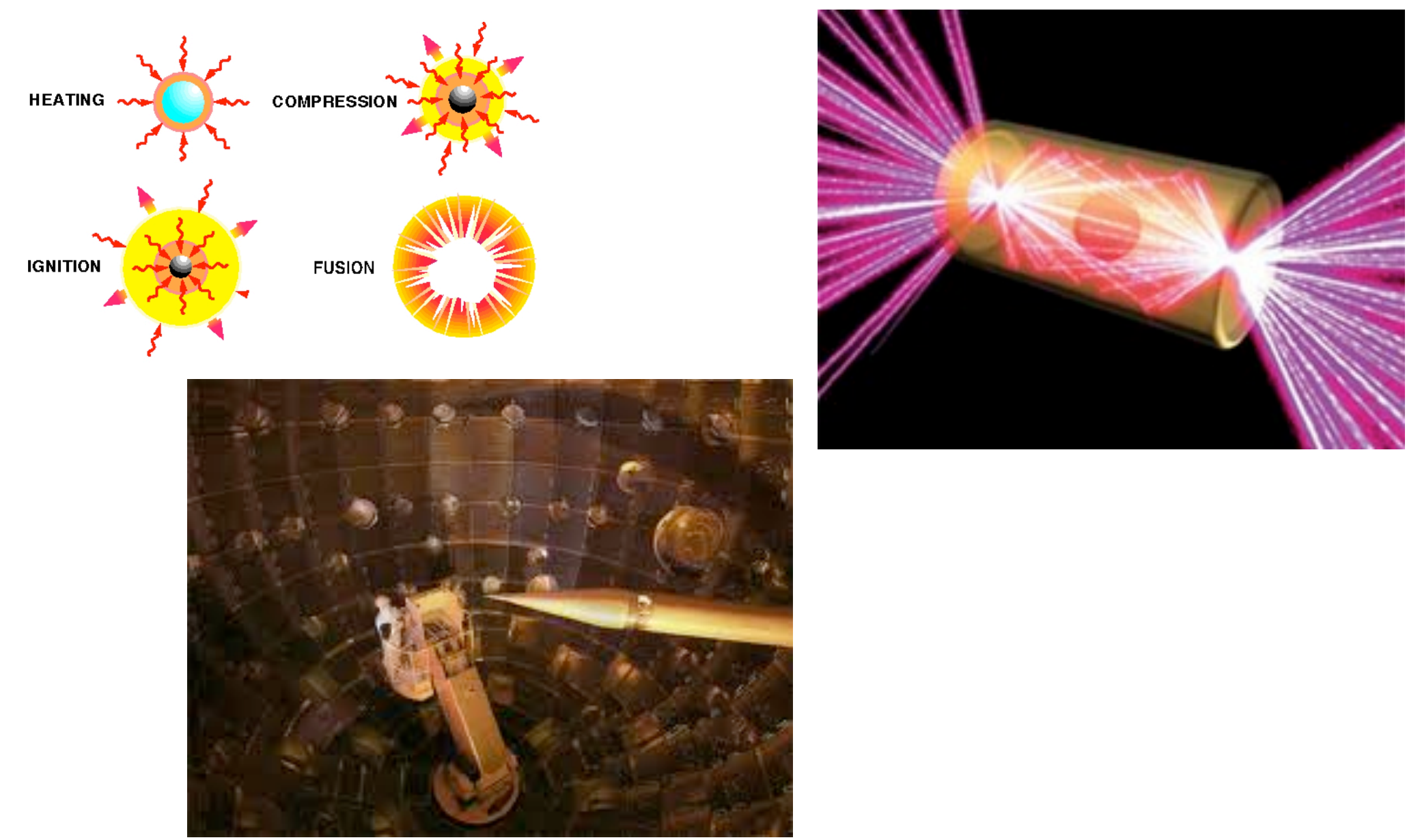


\section{Alternative concepts for ICF}

Fast Ignition

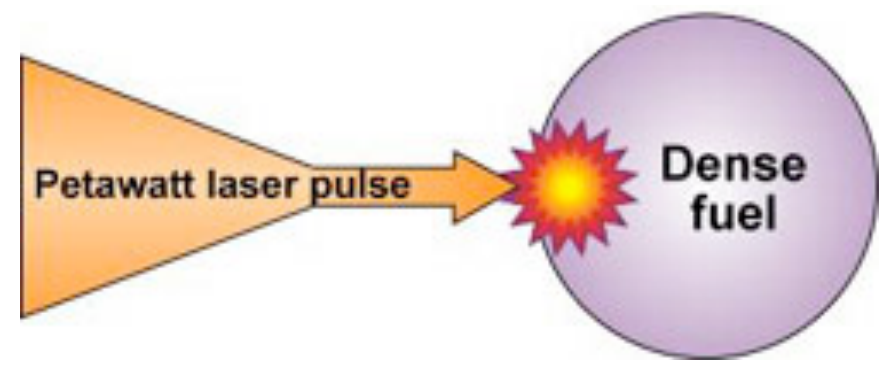

Heavy Ion Fusion

Fission-driven

Z-pinch driven radiation drive

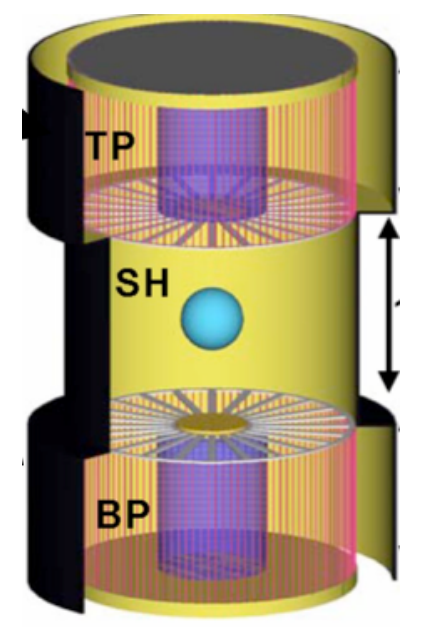

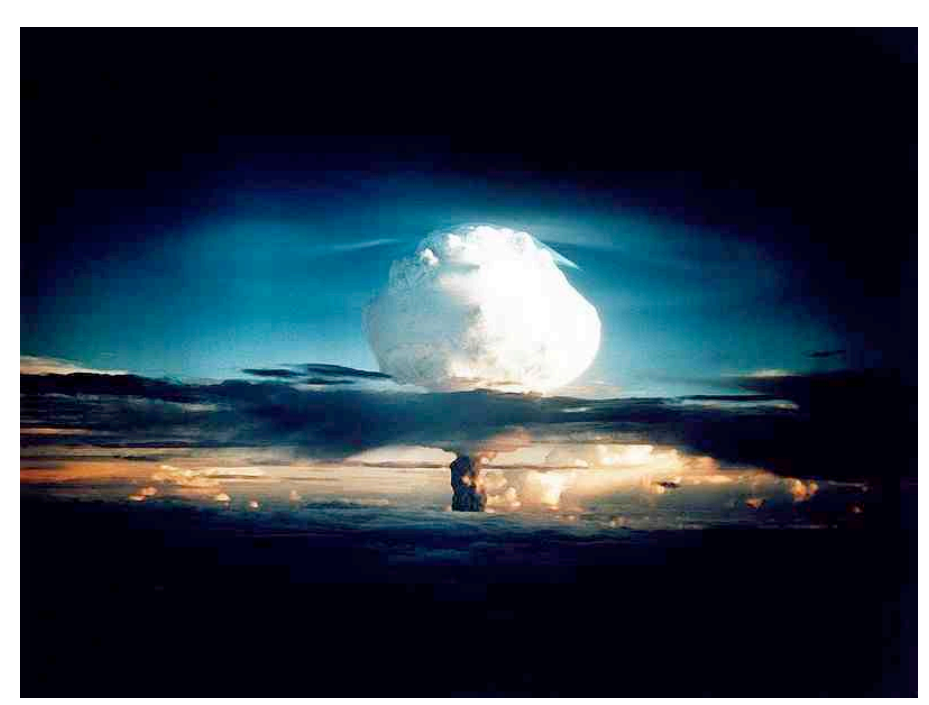

Ivy Mike - First manmade fusion with gain $>1$ 


\section{Magneto-inertial fusion (MIF)}

- MIF regime lies between magnetic and inertial confinement fusion approaches.

- 1-100 megabar pressures, multi-megagauss magnetic fields, ns- $\mu s$ timescales.

- Compared to ICF:

- Embedded magnetic field improves particle confinement, reduces energy transport, confines a-particles.

- This reduces required $\rho R$, implosion speed, and convergence for ignition.

- Enables use of efficient (and inexpensive) pulsed power.

- This relaxes the needed gain for break-even (e.g. $\eta \mathrm{G}=0.5^{\star} 10$ for pulsed power vs. $\eta \mathrm{G}=0.05^{\star} 100$ for lasers).

National Academy of Science IFE Review documents available at: http://fire.pppl.gov/icf nas review 2010.html

Scientific American, May 26, 2011 


\section{Wide range of driver/target combinations.}

U. Rochester LLE

Magnetic transport reduction in ICF target.

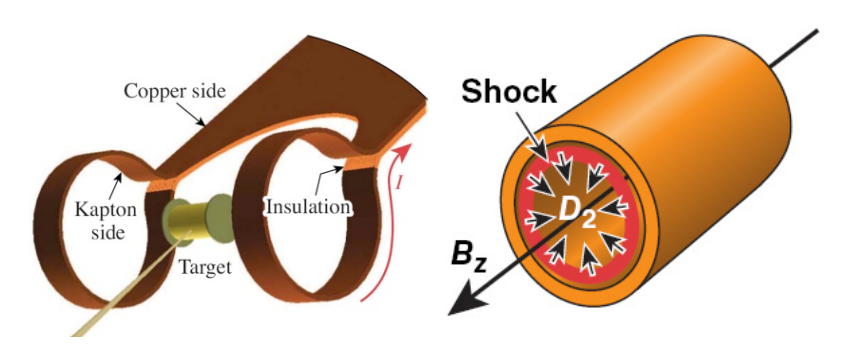

Los Alamos / HyperV

Plasma Liner Experiment (PLX)

Merging plasma jets for remote standoff.

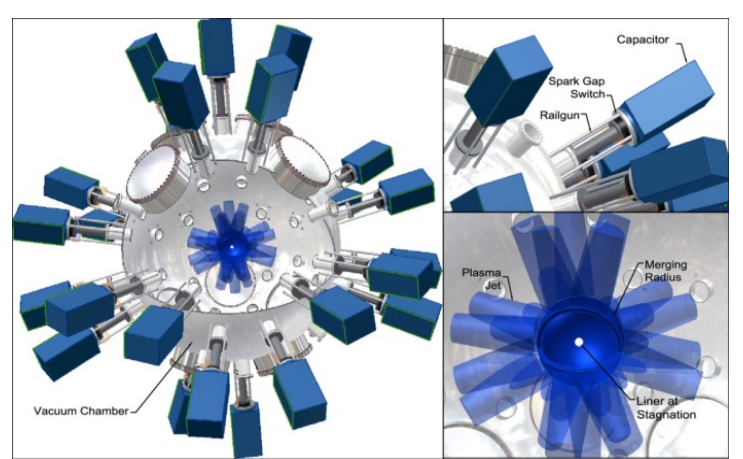

A. G. Lynn, et al, Rev. Sci. Instr. 81, 10E115 (2010)
General Fusion

Spheromak merging and pneumatically driven compression with liquid liner/blanket.

Los Alamos / AFRL Magnetized Target Fusion (MTF) FRX-L/FRCHX + Shiva Star $\sim 20 \mu \mathrm{s}, 0.5 \mathrm{~cm} / \mu \mathrm{s}$ liner implosion Capture

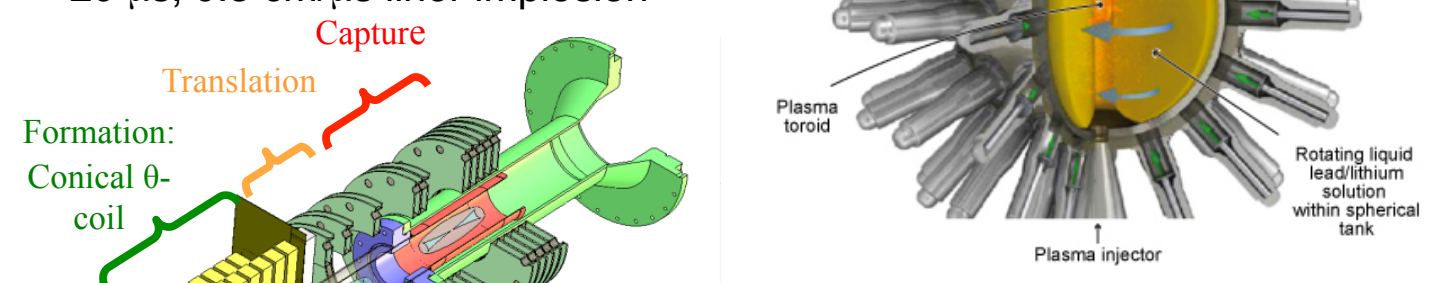

Sandia National Laboratories Magnetized Liner Inertial Fusion (MagLIF) Laser preheated magnetized fuel

Taccetti, Intrator, Wurden et al., Rev. Sci, Instr. 74, 4314 (2003)
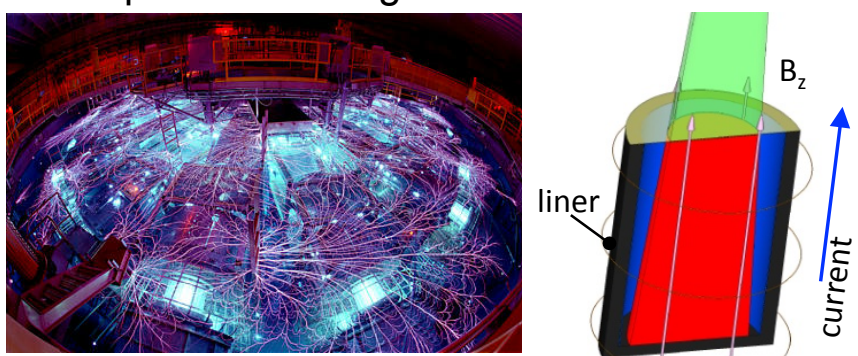

S. A. Slutz, et al., Phys. Plasmas 17, 056303 (2010) 


\section{Non-thermonuclear fusion}

Muon Catalyzed Fusion

- muon replaces electron, "atoms" come close enough to fuse

- muons catalyze many reactions

- "alpha sticking problem"

- difficult to produce muons

\begin{tabular}{|c|c|}
\hline Cold Fusion? & Carnot Efficiency: \\
\hline $\begin{array}{l}\text { - Pons \& Fleischmann } 1989 \\
\text { - unknown process } \\
\text { - lack of evidence }\end{array}$ & $\eta \leq 1-\frac{I_{C}}{T_{H}}$ \\
\hline
\end{tabular}

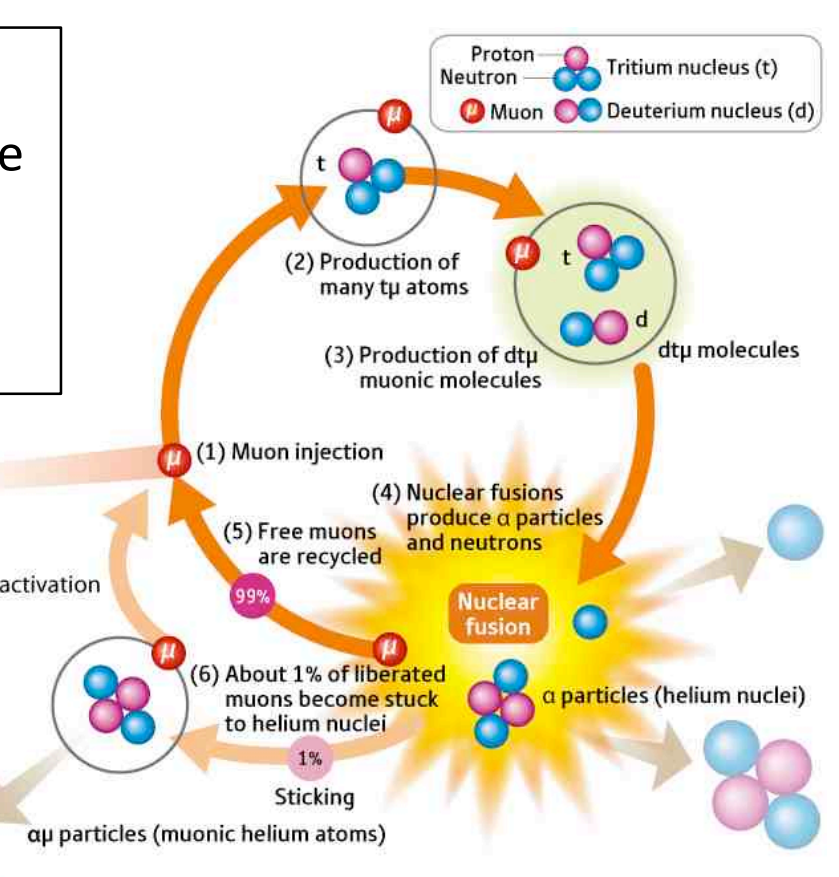

Pycnonuclear fusion

- Highly coupled crystalline strucure

- Lattice vibrations can exceed thermal energy

- high density, relatively cold

- responsible for energy release in carbon core of white dwarfs

Beam-target

- Inefficiencies make concept unsuitable for energy production. 


\section{Other engineering issues}

- Energy extraction - "Blanket"

- Tritium handling

- Tritium breeding

- Cooling

- Interaction of radiation with matter

- Superconducting magnets?

- Plasma-wall interactions

- Economic power generation. 


\section{Fission-Fusion Hybrid}

- $\quad$ No need for $Q>1$

- Waste transmutation

- Subcritical reactor 


\section{Useful References}

- "Fusion" by Weston Stacey

- "The Physics of Inertial Fusion" by S. Atzeni and J. Meyer-ter-vehn 


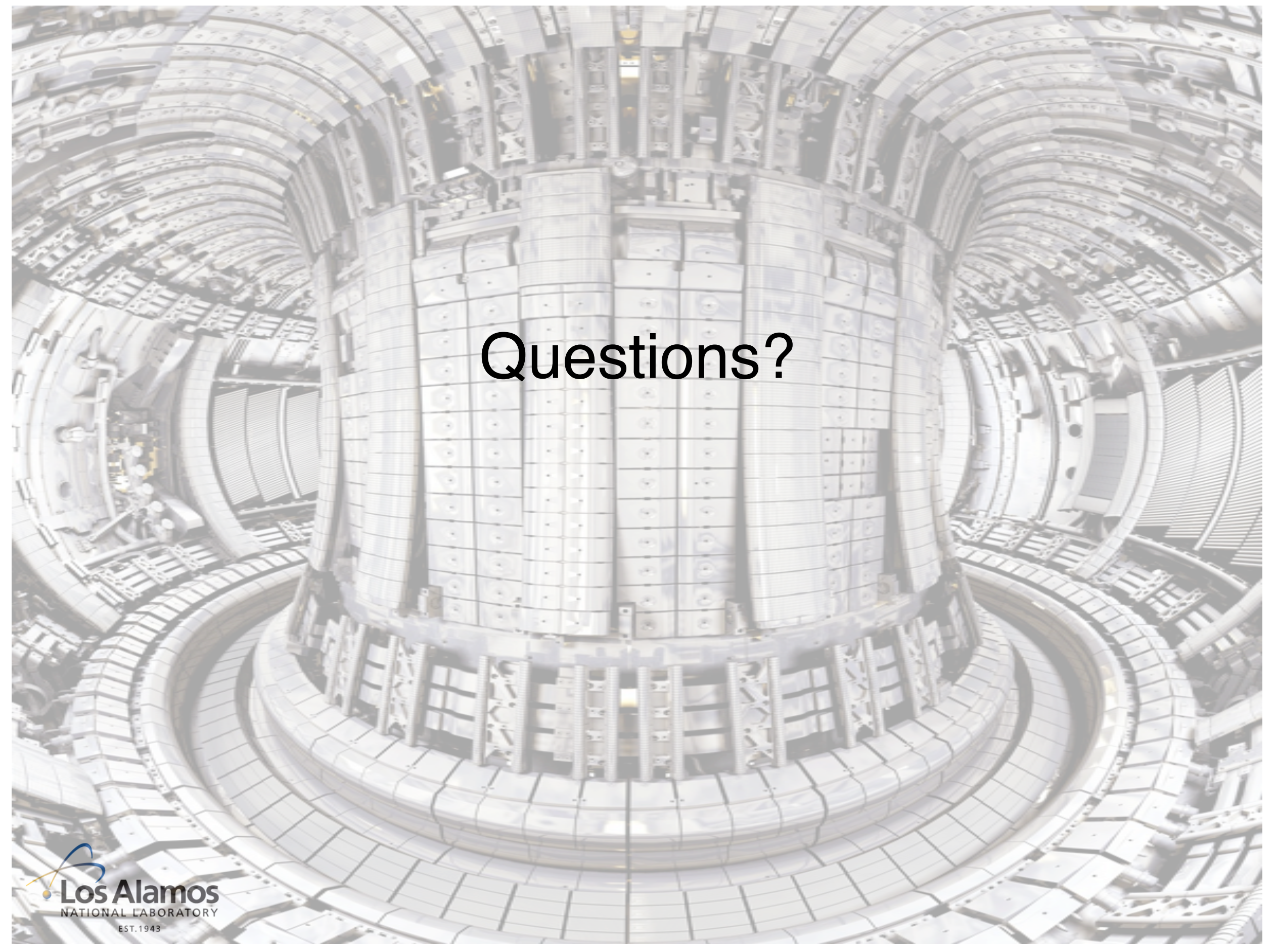




\title{
A Gentle Introduction to Plasma Kinetic Simulation
}

\author{
Bill Daughton
}

Plasma Theory \& Applications, XCP-6

\author{
July II, 20I 2 \\ CNLS - Student Seminar
}




\section{How do we describe these various plasmas?}

\section{Kinetic}

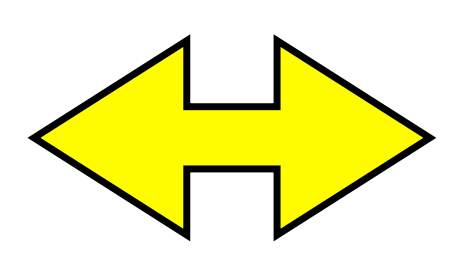

Fluid

Focus of this talk:

I. Theoretical framework for plasmas

2. Describe kinetic PIC method

3. Give some examples 


\section{Start with something familiar and consider air in this room}

1. How fast do particles move?

$$
V_{\text {therm }}=\left(\frac{k_{B} T}{m}\right)^{1 / 2}
$$

2. How far does it travel before colliding with another atom?

- Mean free path

Density Cross-section $\sigma \approx \pi R^{2}$
For air in this room:

$$
n \approx 2 \times 10^{19} \text { atoms }
$$

$L \approx 0.002 \mathrm{~mm}$

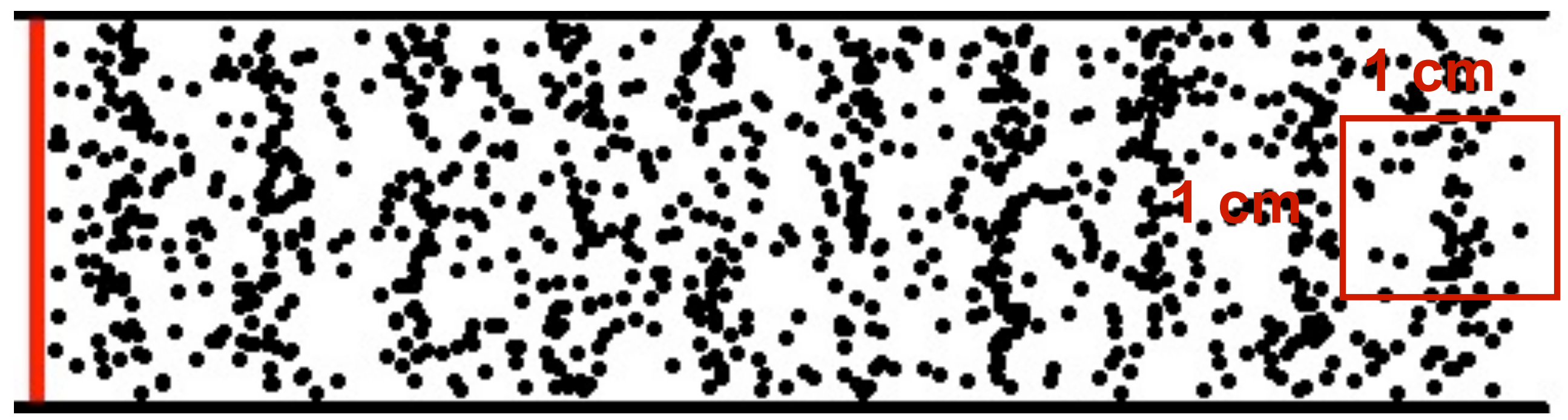

$10^{19}$ atoms 


\section{Conditions for sound waves to occur}

\section{Mean free path
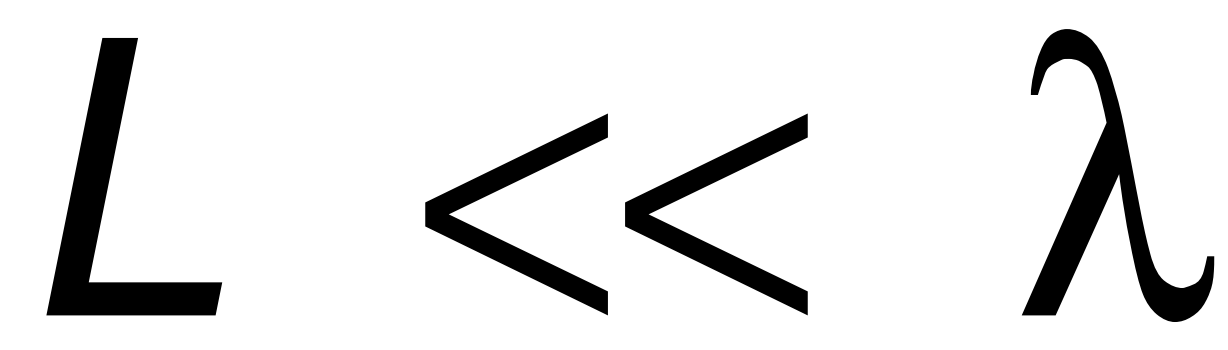 \\ Wavelength}

$L \approx 2 \times 10^{-4} \mathrm{~cm}=0.002 \mathrm{~mm}$

Range of human hearing

Width of Human hair

$0.1 \mathrm{~mm}$

$$
\begin{aligned}
& v \approx 20 \mathrm{~Hz} \circledast 20,000 \mathrm{~Hz} \\
& \lambda \approx 20 \mathrm{~m} \AA 2 \mathrm{~cm}
\end{aligned}
$$

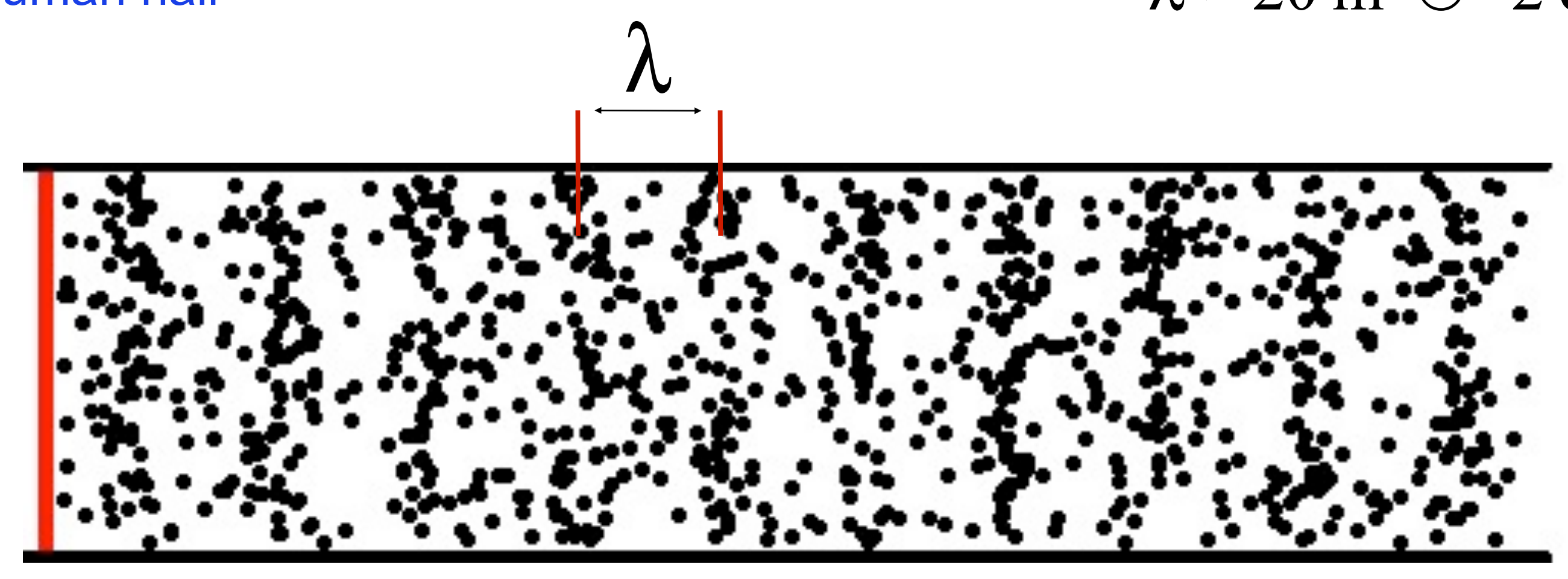


What equations describe a plasmas?

First, we need to consider how charged particles interact 
In a plasma - Coulomb interactions are screened

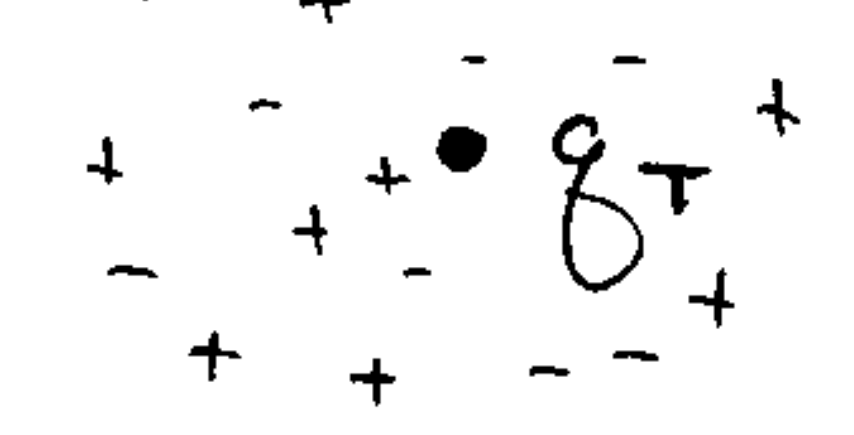

$$
\begin{aligned}
& \nabla \cdot \vec{E}=-\nabla^{2} \phi=4 \pi e\left(n_{i}-n_{e}\right)+4 \pi g_{r} \delta(\vec{r}) \\
& n_{e}=n_{0} \exp \left(\frac{e \phi}{T_{e}}\right) \approx n_{0}\left[1+\frac{e \phi}{T_{e}}+\cdots\right] \\
& n_{i}=n_{0} \exp \left(-\frac{e \phi}{T_{i}}\right) \approx n_{0}\left[1-\frac{e \phi}{T_{i}}+\cdots\right. \\
& \phi=\frac{q_{T}}{r} \exp \left(-\frac{r}{\lambda_{D}}\right) \quad \frac{1}{\lambda_{D}^{2}}=4 \pi e^{2} n_{o}\left(\frac{1}{T_{e}}+\frac{1}{T_{i}}\right)
\end{aligned}
$$

Debye Length 
Huge number of particles within Debye length

$$
\begin{aligned}
& \Lambda \equiv n_{0} \lambda_{D}^{3} \gg 1 \quad \text { Plasma P } \\
& \Gamma=\frac{e \phi}{T_{e}}=\frac{e^{2}}{r k T} \propto \frac{1}{\Lambda_{s}^{2 / 3}}
\end{aligned}
$$

Plasma Parameter

$\Gamma \ll 1 \leadsto$ weakly coupled plasma with many particles in a Debye cube

$\Gamma \geq 1 \backsim$ strongly coupled plasma or sometimes called "Coulomb liquid" on "dense plasma" 


\title{
Collisions in Plasmas?
}

\author{
Rutherford \\ Cross section \\ $\sigma \propto \frac{1}{v^{4}}$
}

\section{Collision \\ Frequency}

$$
\nu=\langle\sigma v\rangle \propto \frac{n}{T^{3 / 2}}
$$

Thermal Velocity

$C_{e}=(2 T / m)^{1 / 2}$

\begin{tabular}{|c|c|c|c|c|c|c|c|}
\hline Application & $n_{e}\left(\mathrm{~cm}^{-3}\right)$ & $T(\mathrm{eV})$ & $\lambda_{D e}(\mathrm{~cm})$ & $n_{e} \lambda_{D e}^{3}$ & $\omega_{p e}$ & $\nu_{e}$ & $C_{e}(\mathrm{~cm} / \mathrm{sec})$ \\
\hline Earth's Magnetotail & 1 & 800 & $2.1 \times 10^{4}$ & $9.3 \times 10^{12}$ & $5.6 \times 10^{4}$ & $3.9 \times 10^{-9}$ & $1.2 \times 10^{9}$ \\
Solar Corona & $10^{7}$ & 100 & 2.3 & $1.3 \times 10^{8}$ & $1.8 \times 10^{8}$ & 0.60 & $4.2 \times 10^{8}$ \\
Tokamak & $10^{14}$ & $10^{4}$ & $7.4 \times 10^{-3}$ & $4.1 \times 10^{7}$ & $5.6 \times 10^{11}$ & $5 \times 10^{3}$ & $4.2 \times 10^{9}$ \\
Laser Plasma & $10^{20}$ & 500 & $1.7 \times 10^{-6}$ & 460 & $5.6 \times 10^{14}$ & $1.9 \times 10^{11}$ & $9.4 \times 10^{8}$ \\
\hline
\end{tabular}




\section{What equations describe a plasma?}

$$
\begin{array}{r}
\frac{\partial f_{s}}{\partial t}+\mathbf{v} \bullet \frac{\partial f_{s}}{\partial \mathbf{x}}+\frac{q_{s}}{m_{s}}\left(\mathbf{E}+\frac{\mathbf{v} \times \mathbf{B}}{c}\right) \cdot \frac{\partial f_{s}}{\partial \mathbf{v}}:=0 \quad \begin{array}{c}
\text { Vlasov } \\
\varepsilon=0
\end{array} \\
f_{s}(\mathbf{x}, \mathbf{v}, t)-\frac{\text { Number of particles }}{\text { Unit volume of phase space }}
\end{array}
$$

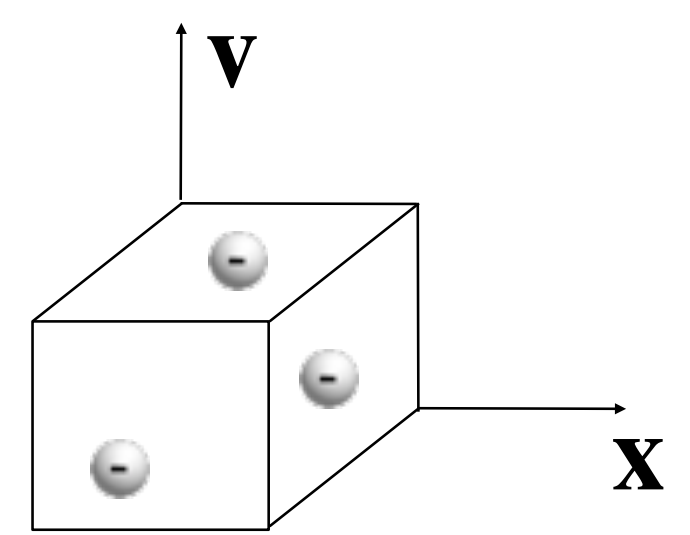

$$
\text { Small Parameter }-\varepsilon=\frac{1}{n \lambda_{D}^{3}} \sim 10^{-6} \rightarrow 10^{-12}
$$

\section{Maxwell's Equations}

$$
\begin{aligned}
& \rho=\sum_{s} q_{s} \int f_{s} d \mathbf{v} \\
& \mathbf{J}=\sum q_{s} \int \mathbf{v} f_{s} d \mathbf{v} \\
& \nabla \bullet \mathbf{B}=0 \\
& \nabla \times \mathbf{B}=\frac{4 \pi}{c} \mathbf{J}+\frac{1}{c} \frac{\partial \mathbf{E}}{\partial t} \\
& \nabla \bullet \mathbf{E}=4 \pi \rho \\
& \nabla \times \mathbf{E}=-\frac{1}{c} \frac{\partial \mathbf{B}}{\partial t}
\end{aligned}
$$




\section{Vlasov-Maxwell Theory}

\section{Vlasov}

$\frac{\partial f_{s}}{\partial t}+\mathbf{v} \bullet \frac{\partial f_{s}}{\partial \mathbf{x}}+\frac{q_{s}}{m_{s}}\left(\mathbf{E}+\frac{\mathbf{v} \times \mathbf{B}}{c}\right) \cdot \frac{\partial f_{s}}{\partial \mathbf{v}}=0$

- Coupled by first 2 moments

\section{Maxwell}

$$
\begin{array}{ll}
\nabla \bullet \mathbf{B}=0 & \nabla \times \mathbf{B}=\frac{4 \pi}{c} \mathbf{J}+\frac{1}{c} \frac{\partial \mathbf{E}}{\partial t} \\
\nabla \bullet \mathbf{E}=4 \pi \rho & \nabla \times \mathbf{E}=-\frac{1}{c} \frac{\partial \mathbf{B}}{\partial t}
\end{array}
$$

- Complete description of collisionless plasma

- Very difficult to solve - 6D phase space!

- Fluid description is much easier 


\section{Fluid Description of Plasma}

$$
\begin{aligned}
& \text { Density } \longrightarrow n_{s}=\int f_{s} d \mathbf{v} \\
& \text { Velocity } \longrightarrow \mathbf{U}_{s}=\int \mathbf{v} f_{s} d \mathbf{v} \\
& \text { Pressure } \longrightarrow \mathbf{P}_{s}=m_{s} \int\left(\mathbf{v}-\mathbf{U}_{s}\right)\left(\mathbf{v}-\mathbf{U}_{s}\right) f_{s} d \mathbf{v}
\end{aligned}
$$

Take velocity space moments of the Vlasov Equation:

$$
\text { Mass conservation } \longrightarrow \frac{\partial n_{s}}{\partial t}+\nabla \bullet\left(n_{s} \mathbf{U}_{s}\right)=0
$$

Momentum conservation

$$
\longrightarrow m_{s} n_{s} \frac{d \mathbf{U}_{s}}{d t}=-\nabla \bullet \mathbf{P}_{s}+q_{s} n_{s}\left(\mathbf{E}+\frac{\mathbf{U}_{s} \times \mathbf{B}}{c}\right)
$$




\section{MHD Model}

$$
\begin{aligned}
& \frac{\partial \rho}{\partial t}+\nabla \bullet(\rho \mathbf{U})=0 \\
& \rho \frac{d \mathbf{U}}{d t}=-\nabla \mathbf{P}+\frac{\mathbf{J} \times \mathbf{B}}{c} \\
& \mathbf{E}+\frac{\mathbf{U} \times \mathbf{B}}{c}=\eta \mathbf{J} \\
& \frac{d}{d t}\left(\frac{P}{\rho^{\gamma}}\right)=0 \\
& \nabla \times \mathbf{B}=\frac{4 \pi}{c} \mathbf{J} \\
& \nabla \times \mathbf{E}=-\frac{1}{c} \frac{\partial \mathbf{B}}{\partial t} \\
& \nabla \bullet \mathbf{B}=0
\end{aligned}
$$

\section{Mass conservation}

Momentum conservation

Ohm's Law

Adiabatic equation of state

Ampere's Law

Faraday's Law

$$
\text { Ideal MHD }-\eta=0 \longrightarrow \mathbf{U}_{\perp}=c \frac{\mathbf{E} \times \mathbf{B}}{B^{2}}
$$


What problems need kinetic treatment?

I. kinetic scales are important?

2. Resonant interactions?

3. Don't have a reasonable fluid closure?

4. Need to predict particle acceleration?

How to solve kinetic equation?

I. Directly discretize kinetic equation 2. Monte-Carlo approach - i.e. Particle-in-cell 


\section{PIC = Particle-in-cell}

- Introduce "super-particles" - Lagrangian tracers

- Create spatial grid (cells)

- Interpolate position and velocity of particle onto grid $\rho \vec{J}$

- Compute resulting $E$ and $B$ fields

- Push particles using these self-consistent fields

- Evolution of this system obeys a kinetic equation

Electron o

Ion 0

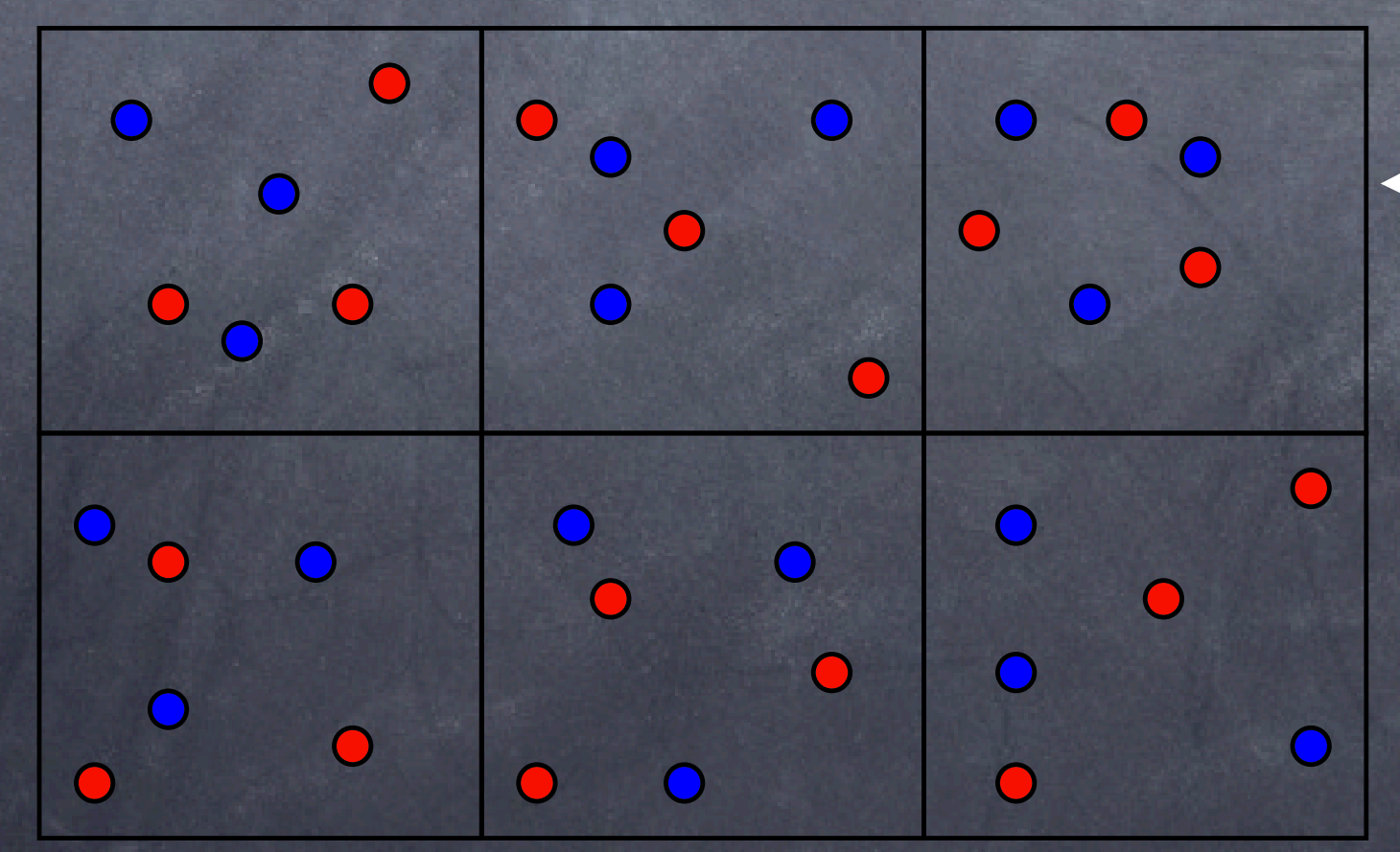

$\longleftarrow$ N particles/cell

For large $\mathrm{N}$, kinetic equation approaches Vlasov 


\section{Easy to make PIC algorithm parallel}
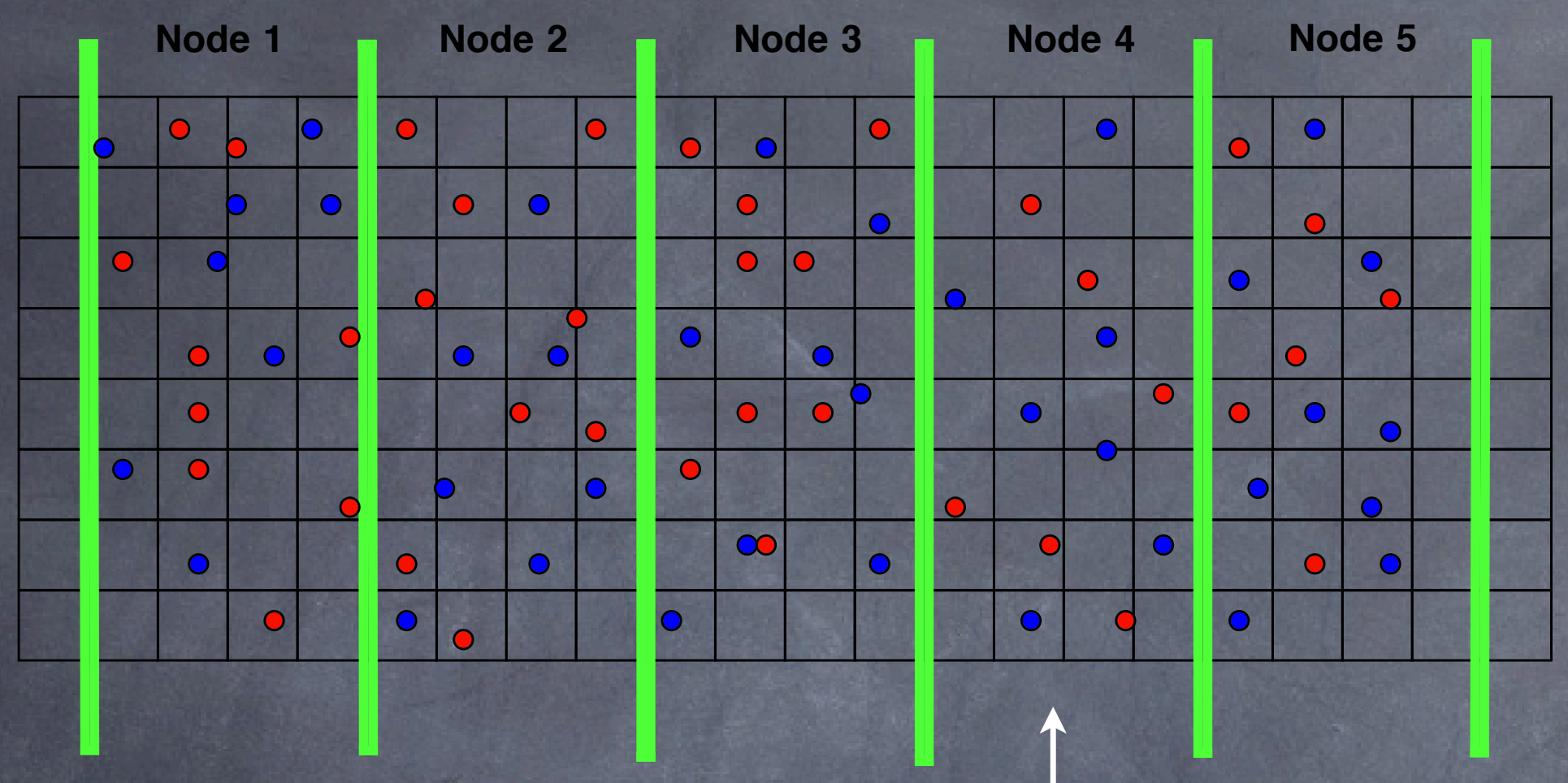

33 - SMP Nodes

2 Opteron CPU's in each 5 GB RAM - 165 GB Total 4 TB Raid Storage Gigabit Ethernet
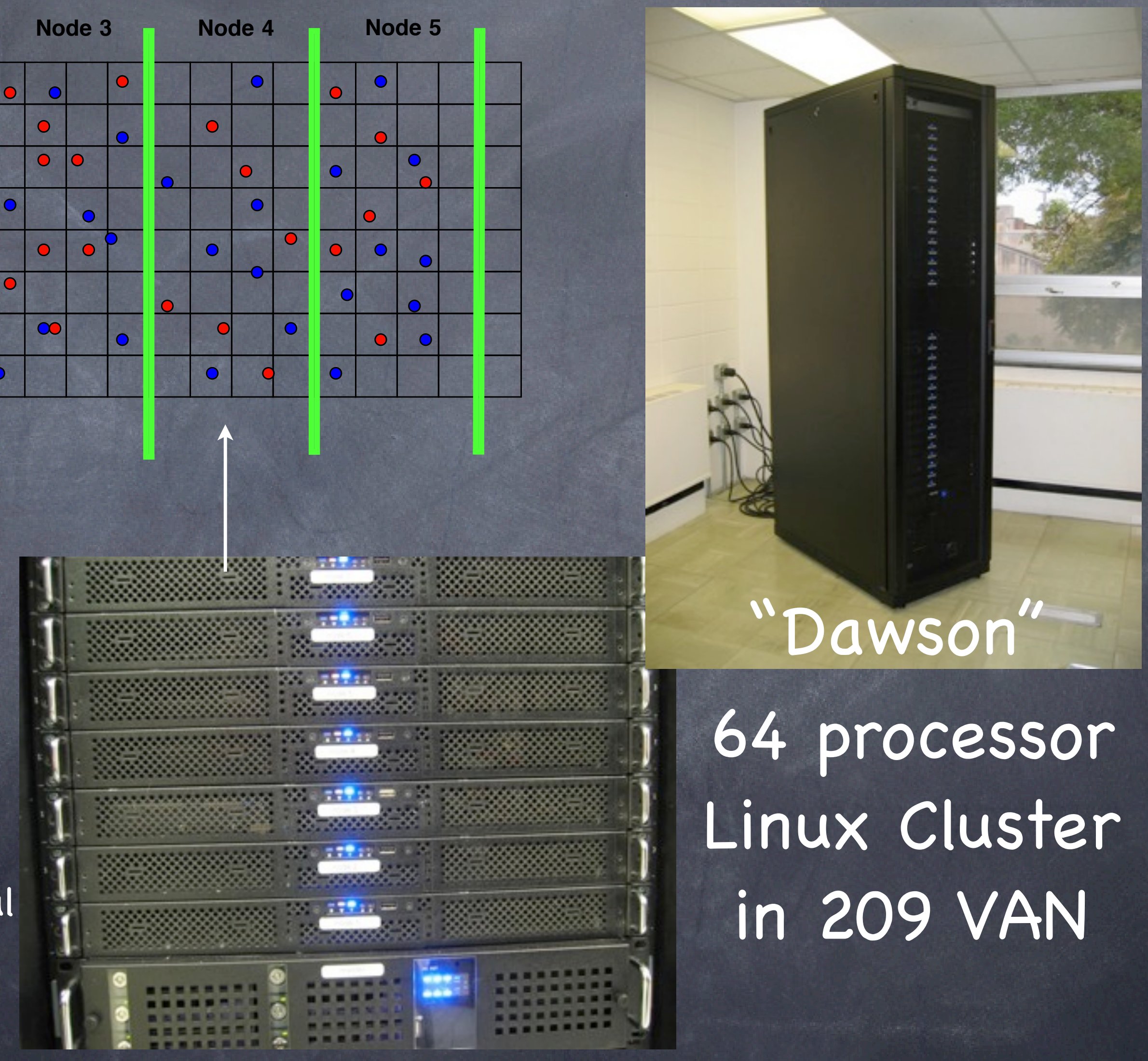

64 processor

Linux Cluster in 209 VAN 


\section{Exponential increase in computing is permitting ever larger kinetic simulations}

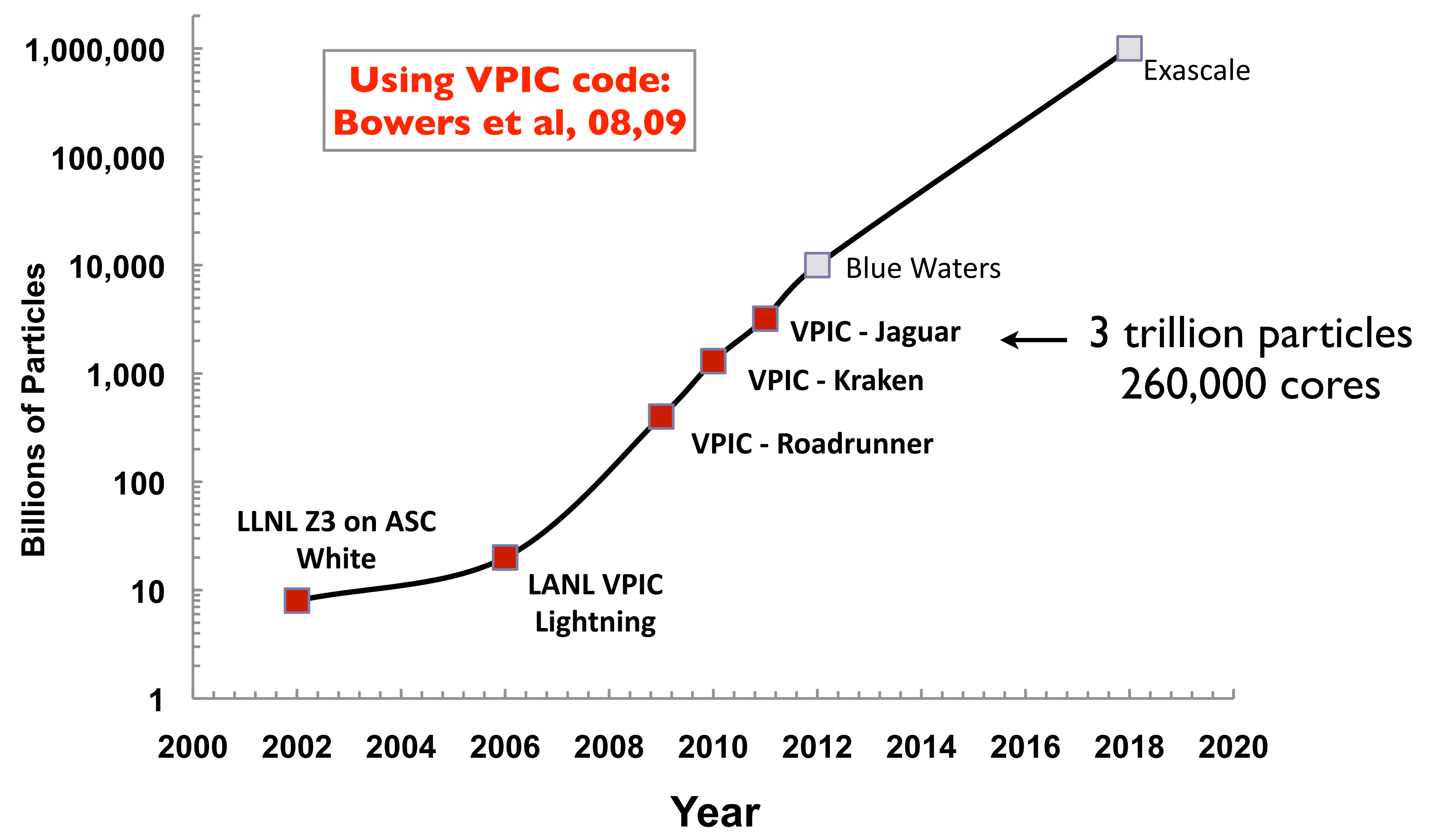




\section{Even so, we usually can't match all experimental parameters}

Depending on the problem - we often choose artificial values for

I. Ion to electron mass ratio

2. Plasma temperature

3. Other dimensionless ratios 


\section{Sample Problem \#I}

Magnetic Reconnection 
At the petascale - global magnetospheric simulations are now feasible with hybrid codes

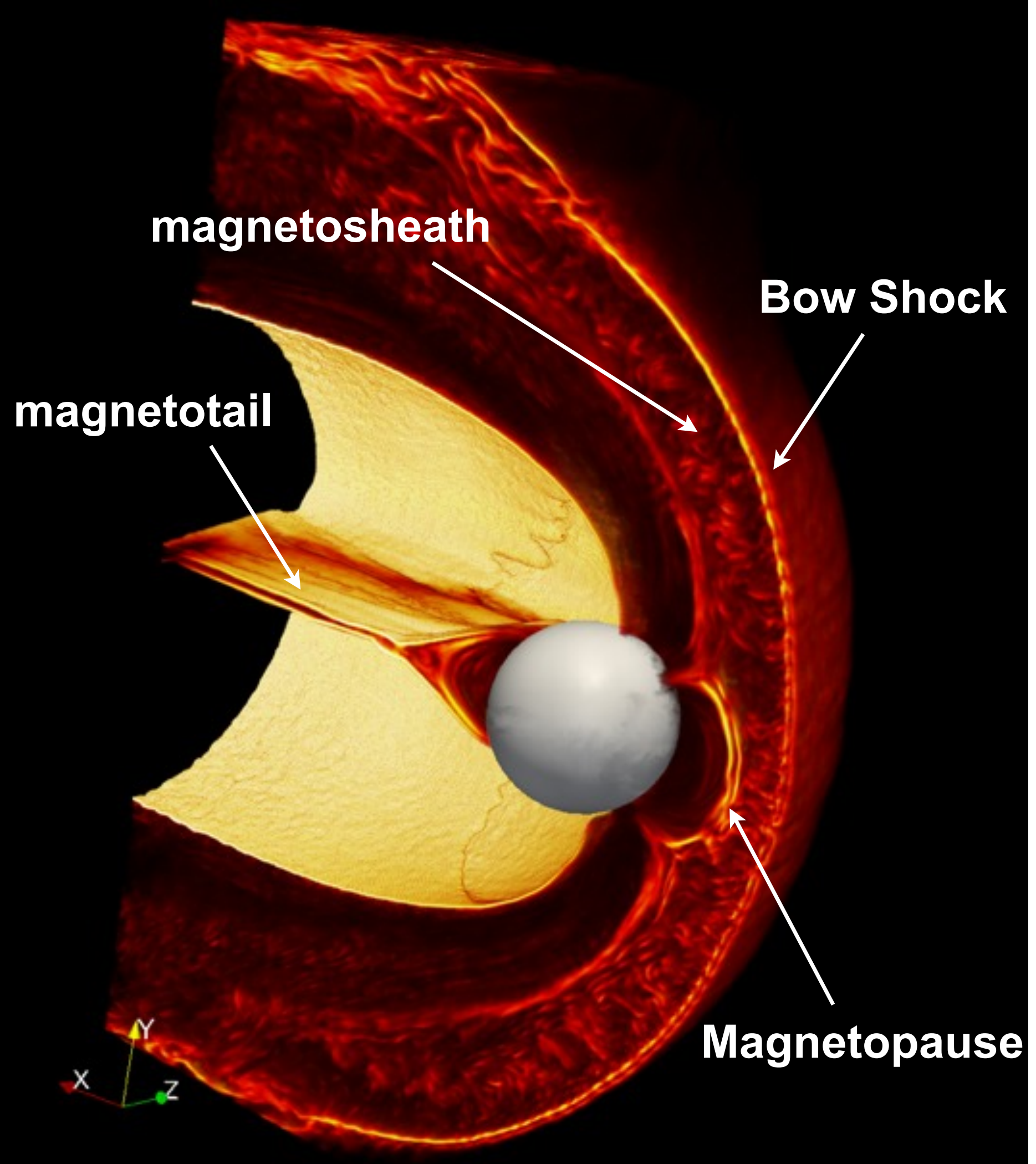

Hybrid offers good description of:

- Collisionless shocks

- Ion kinetic \& FLR effects

- Temperature anisotropy instabilities

- Ion-scale boundary layers

Electrons are massless fluid missing collisionless physics to break frozen-in constraint

Influence of electron physics is the main science goal of NASA's upcoming MMS mission

MMS will launch in 2014 - orbits will be optimized for the magnetopause during first I.5 years of operation

See - Moore, Burch, Daughton, et al, 2012

Hybrid $(2560)^{3}$ cells $\sim 1^{12}$ ions 
High Resolution 3D Hybrid Simulation - $(2560)^{3}$ cells

Fully kinetic simulations possible in smaller region

\section{$100 d_{i} \sim R_{E}$}

$n_{e}$
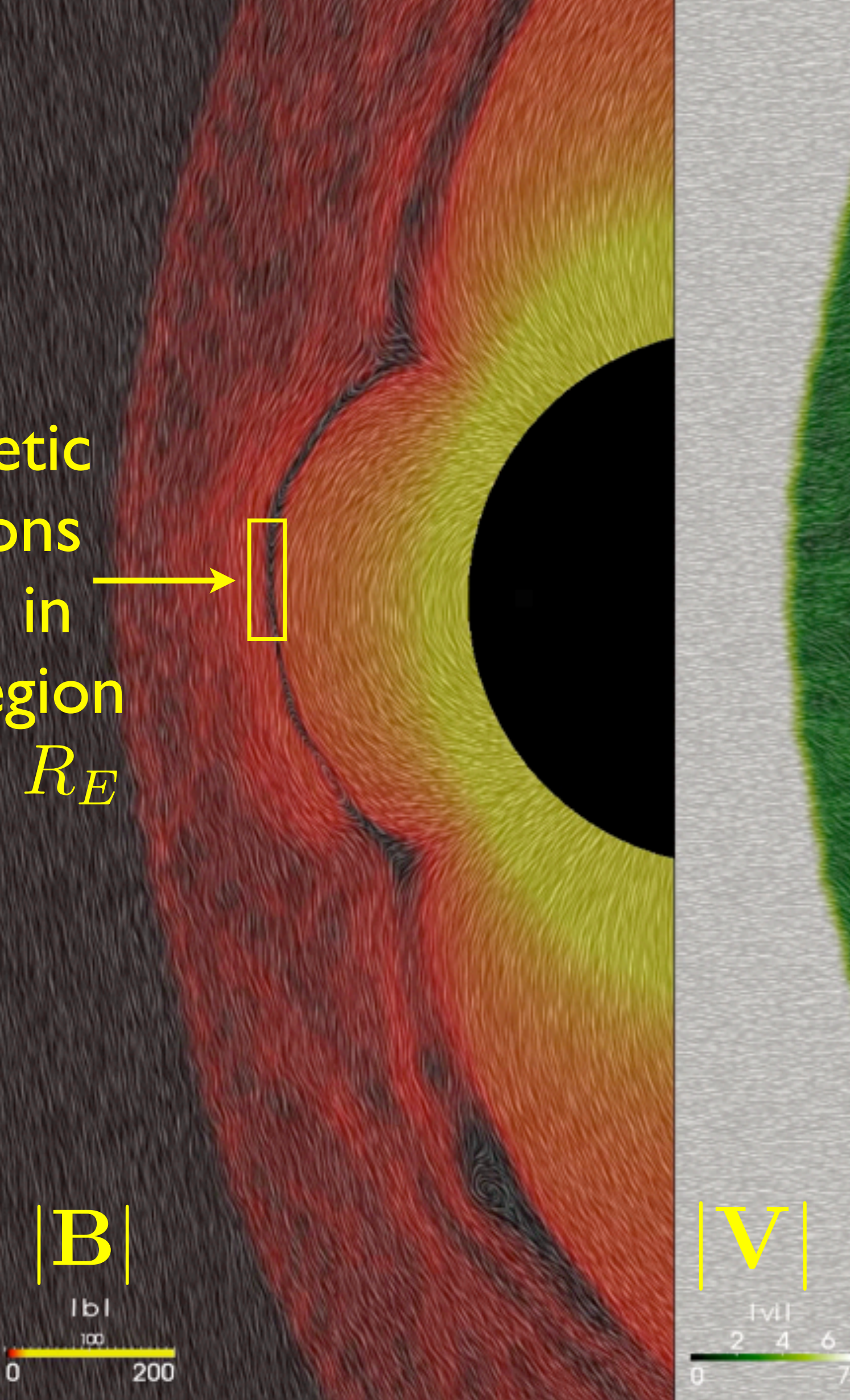


\section{Move 2D slice}

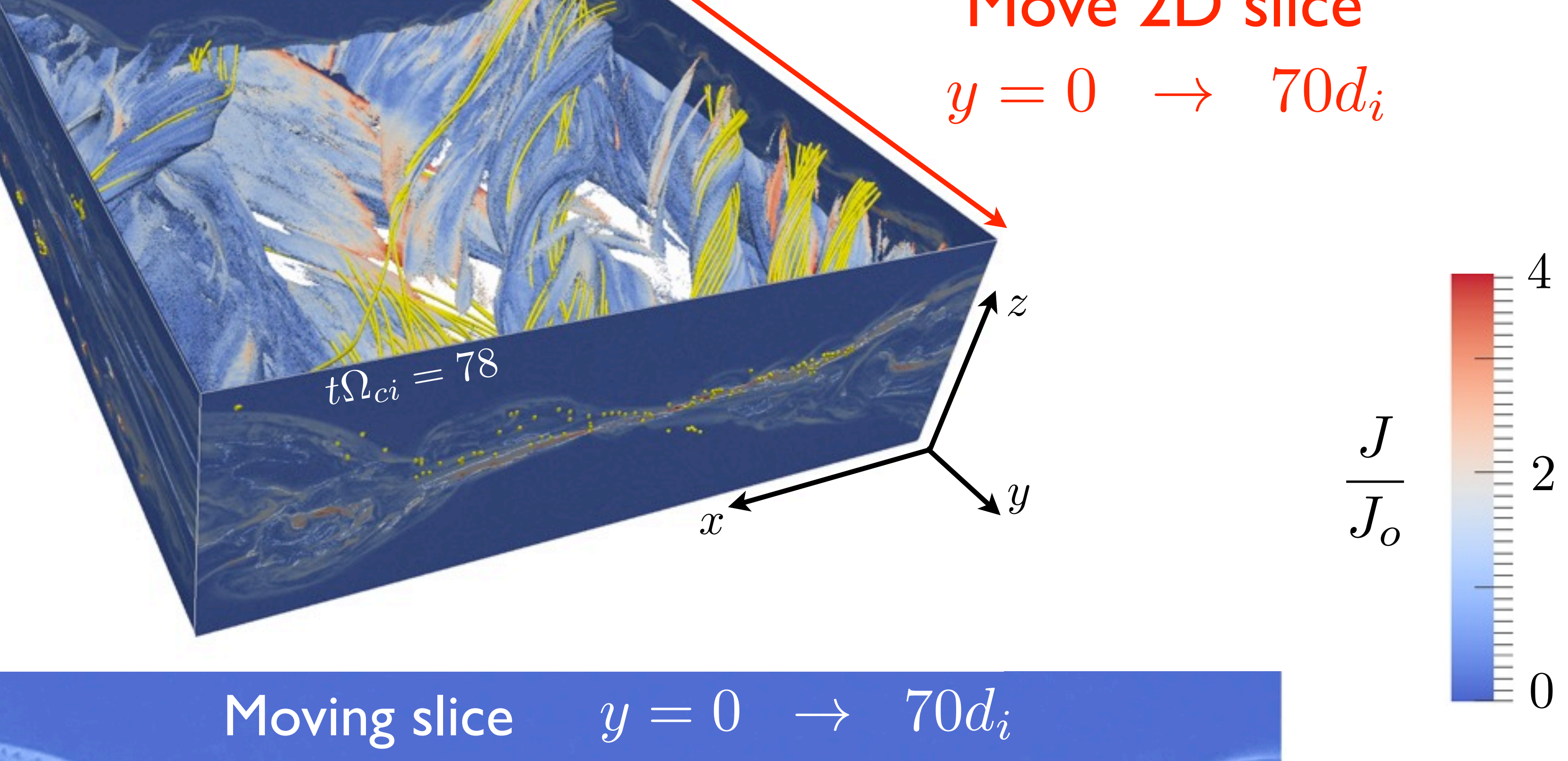

$$
t \Omega_{c i}=78
$$$$
x
$$ 


\section{Sample Problem \#2}

Flow Shear Turbulence 
MHD scale vortices generate current sheets, flux ropes, reconnection \& turbulence

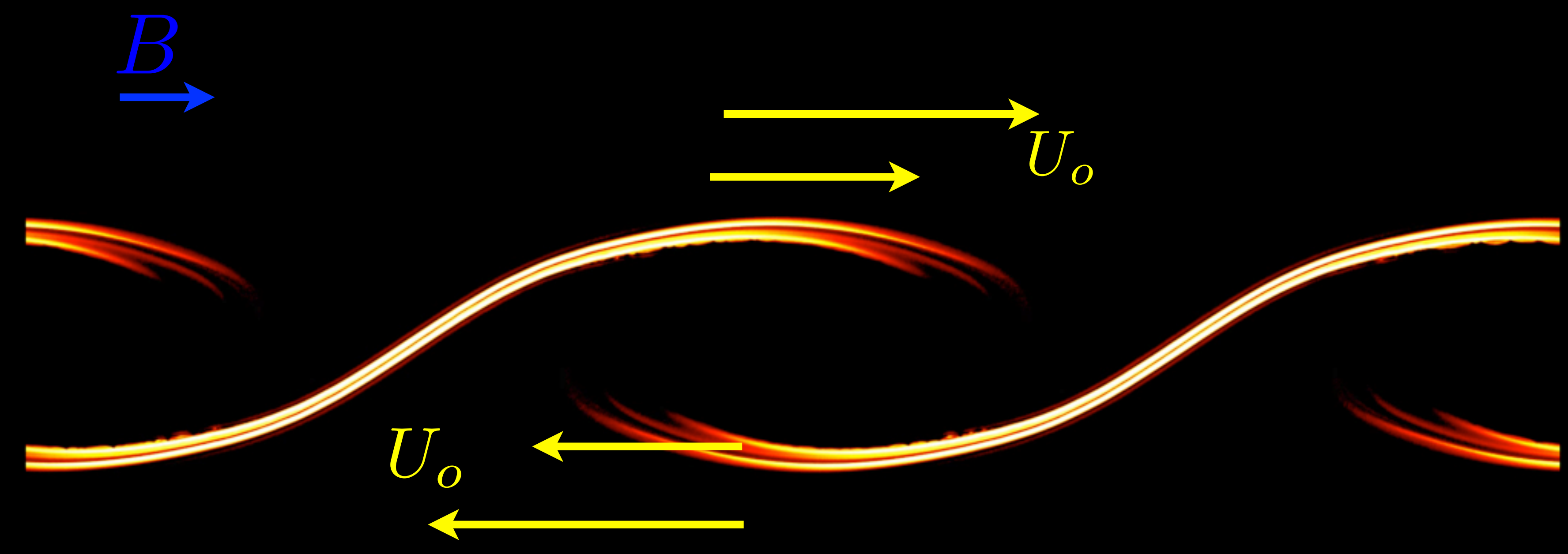

$|\mathrm{J}|$

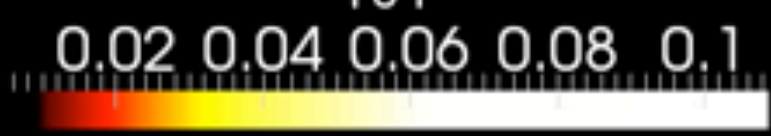
$\begin{array}{ll}0.005 & 0.109\end{array}$

0193

$U_{o}>V_{A} \rightarrow K H \quad m_{i} / m_{e}=100$ 
KH turbulence drives reconnection

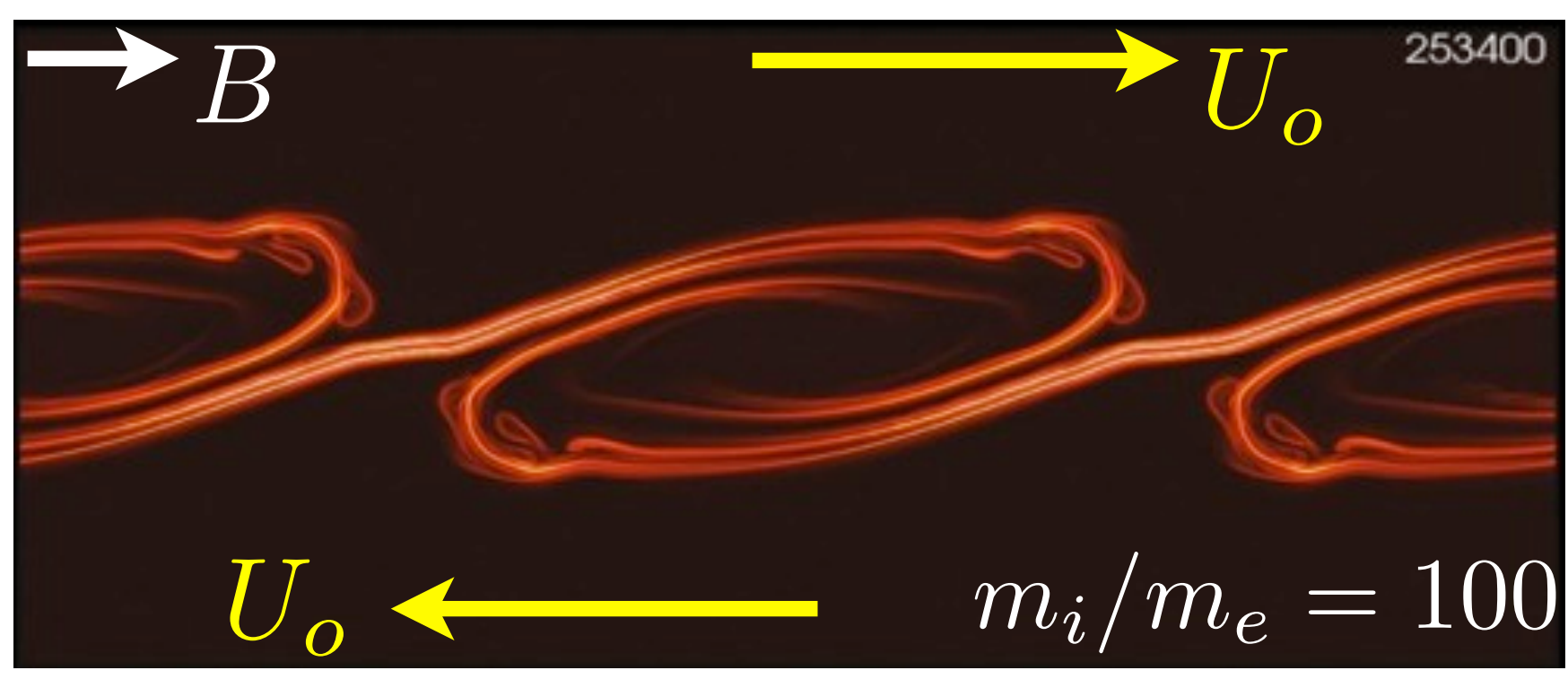

285980
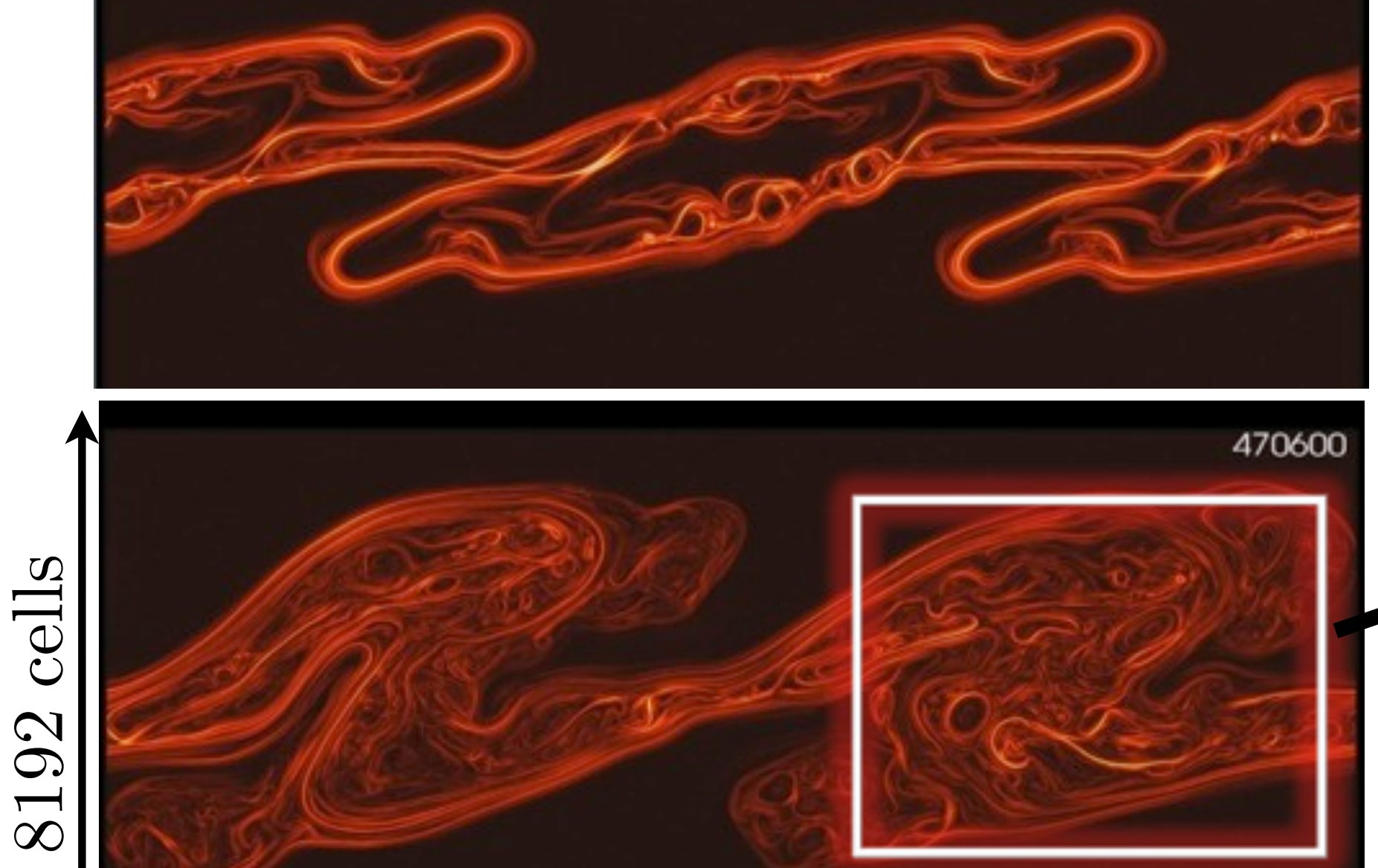

Karimabadi et al, 2012

- Vortex scale $\sim 50 d_{i}$

- Kinetic scale layers

- Secondary tearing \& KH

- Power law spectra

- Electron heating dominant

- In-plane B is crucial
470600

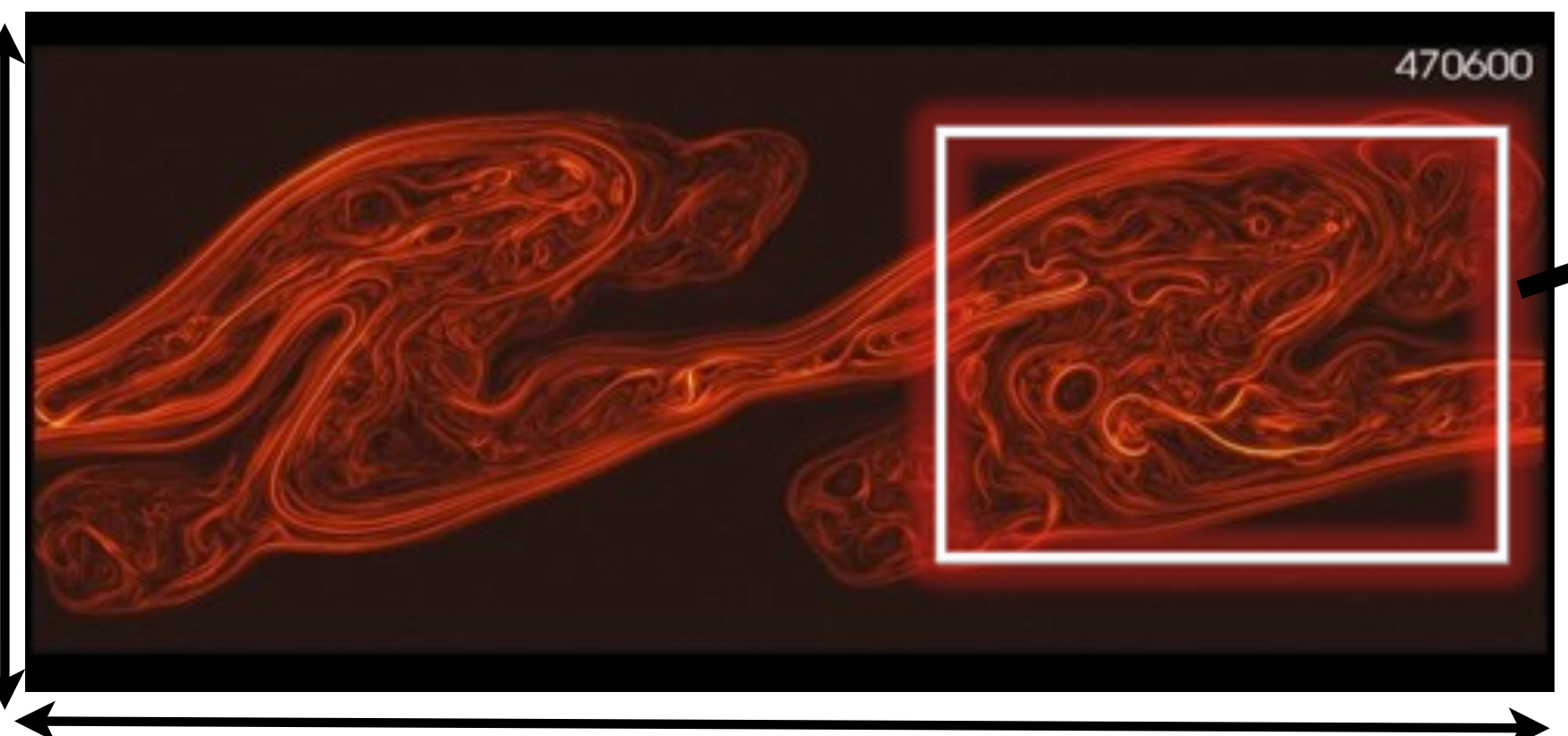

16384 cells $=100 d_{i}$

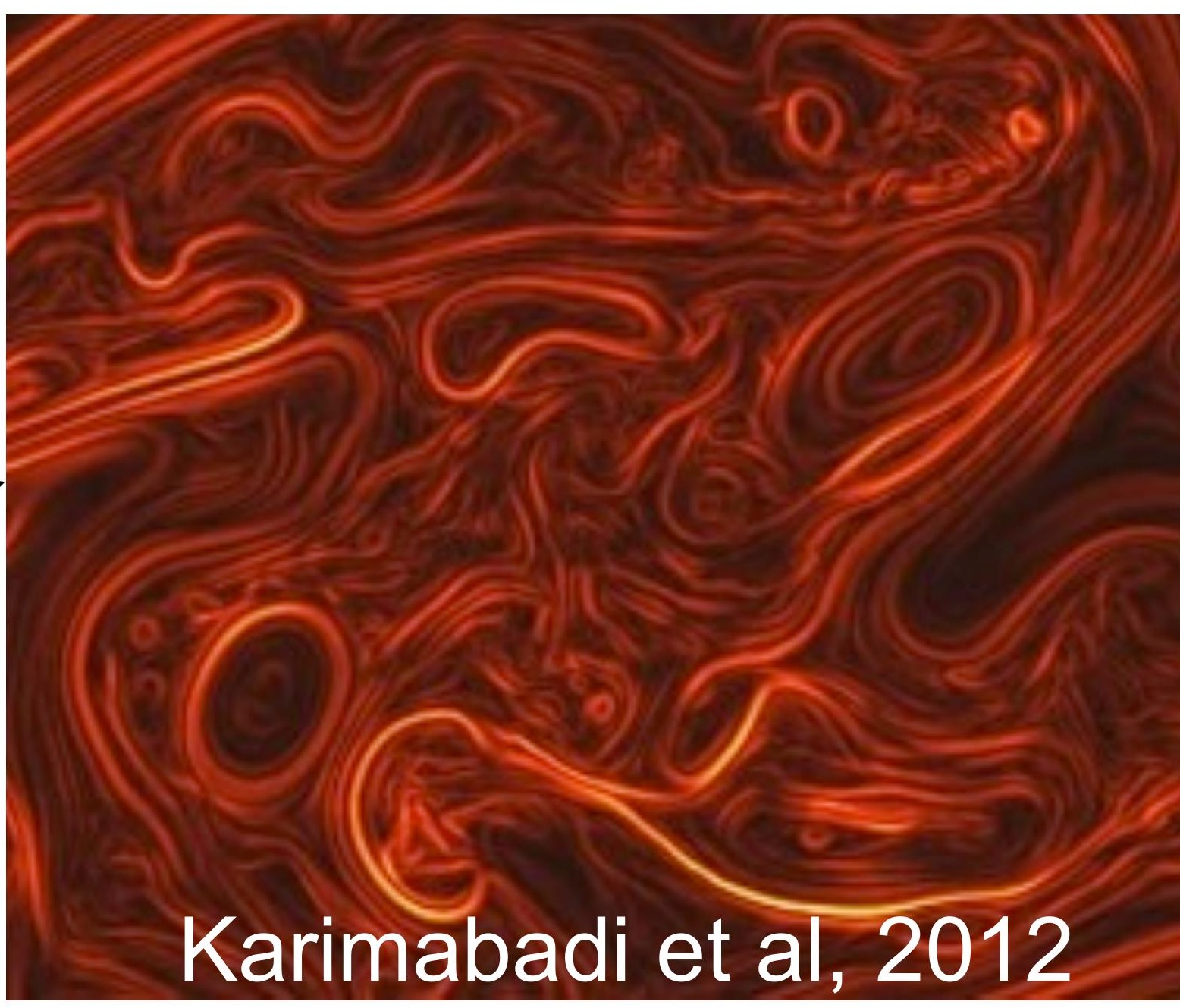


Sample Problem \#3 Laser Wakefield Accelerators 


\section{What is Laser Wakefield Acceleration?}

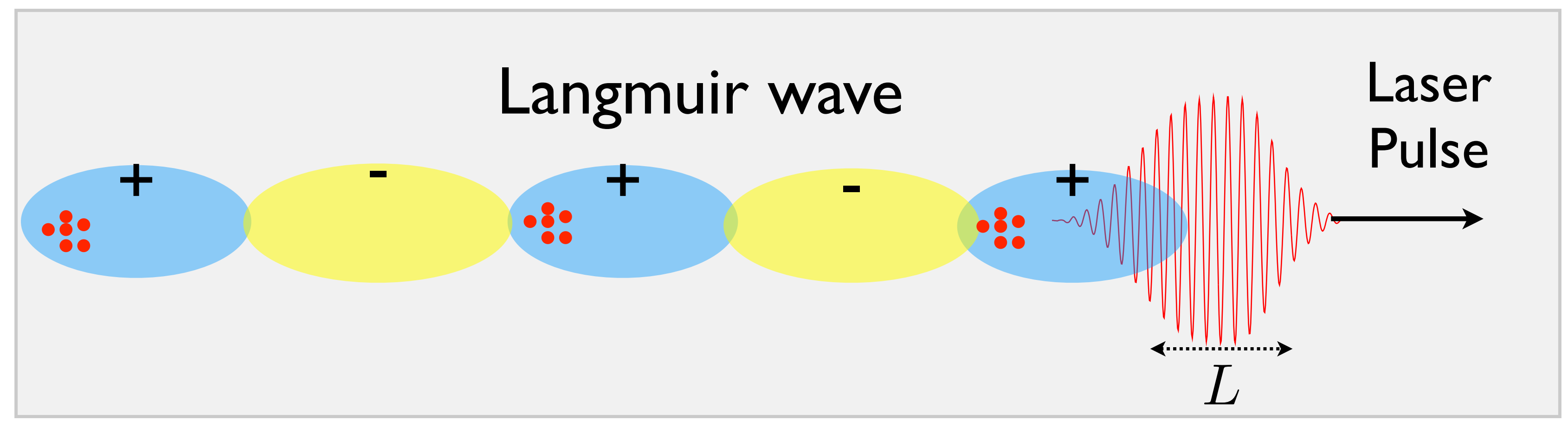

- Plasmas permit huge accelerating fields $(0.1-10) \mathrm{TV} / \mathrm{m}$

- Old idea - Tajima \& Dawson, 1979 - but rapid progress in recent years from experiments, theory \& simulations

- Particularly efficient in the bubble or blowout regime

Two

Requirements:

$$
L<\lambda_{p}=\frac{2 \pi c}{\omega_{p e}}
$$

$a_{o} \gg 1$
Pukhov \& Meyer-Ter-Vehn, 2002

Mangels et al, Nature, 2004

Geddes et al, Nature, 2004

Faure et al, Nature 2004 


\section{Example Simulation}

Dimensionless Amplitude

$$
a_{o}=\frac{e A_{y}}{m_{e} c^{2}}=4
$$

Laser Intensity

$$
I=\frac{m_{e}^{2} c^{3}}{8 \pi e^{2}}\left(\omega_{o} a_{o}\right)^{2}
$$

For $800 \mathrm{~nm}$ laser:

$$
I \approx 2.2 \times 10^{18} a_{o}^{2} \mathrm{~W} / \mathrm{cm}^{2}
$$

Simulation parameters $640 \times 512 \times 512$ cells 8 particles/cell
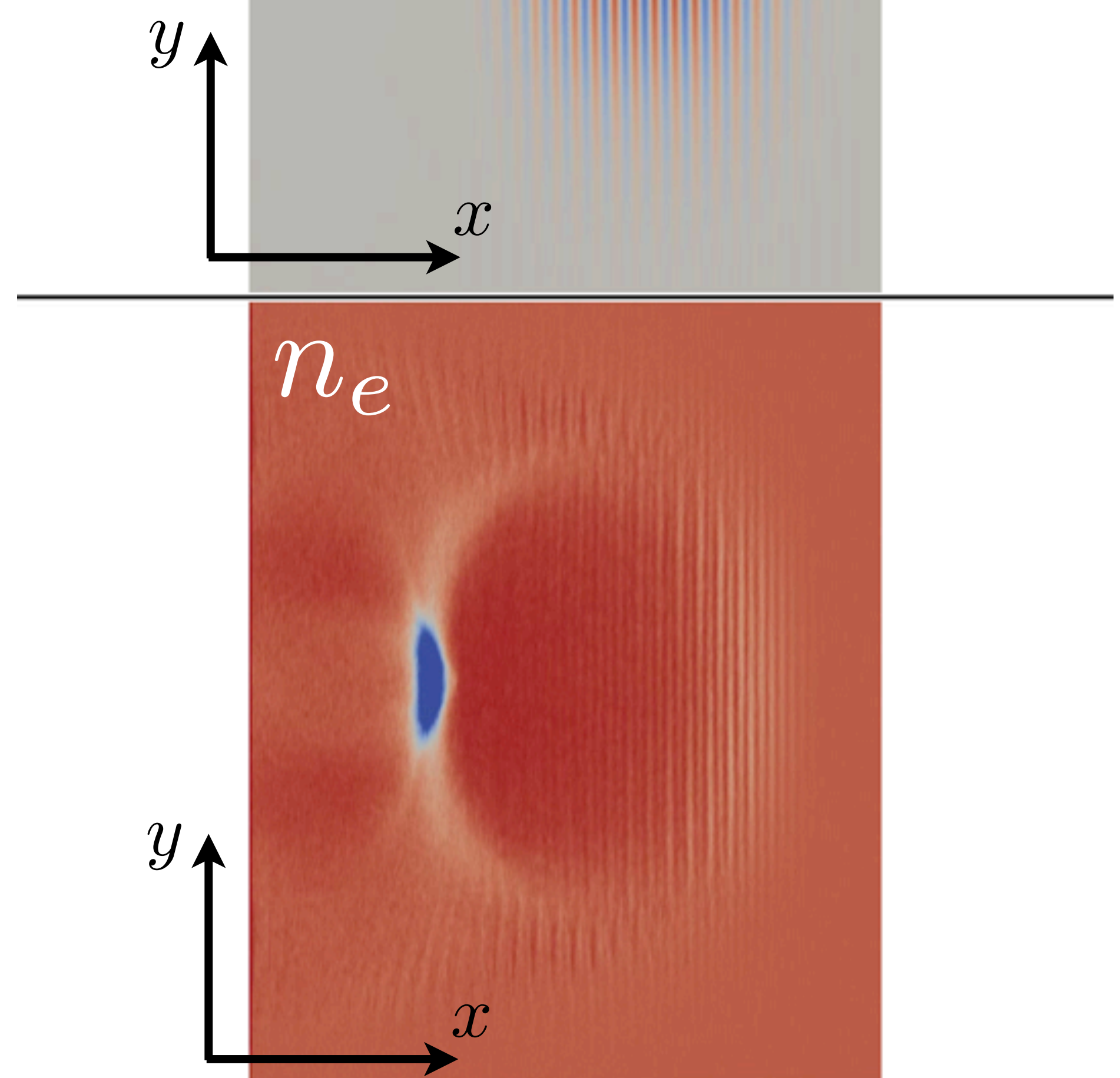


\section{Wakefield acceleration - in the Blowout Regime}




\section{Sample Problem \#4}

Parametric Instabilities

$$
\text { relevant to NIF }
$$

Many recent papers from Lin Yin - XCP-6 


\section{What is Inertial Confinement Fusion?}
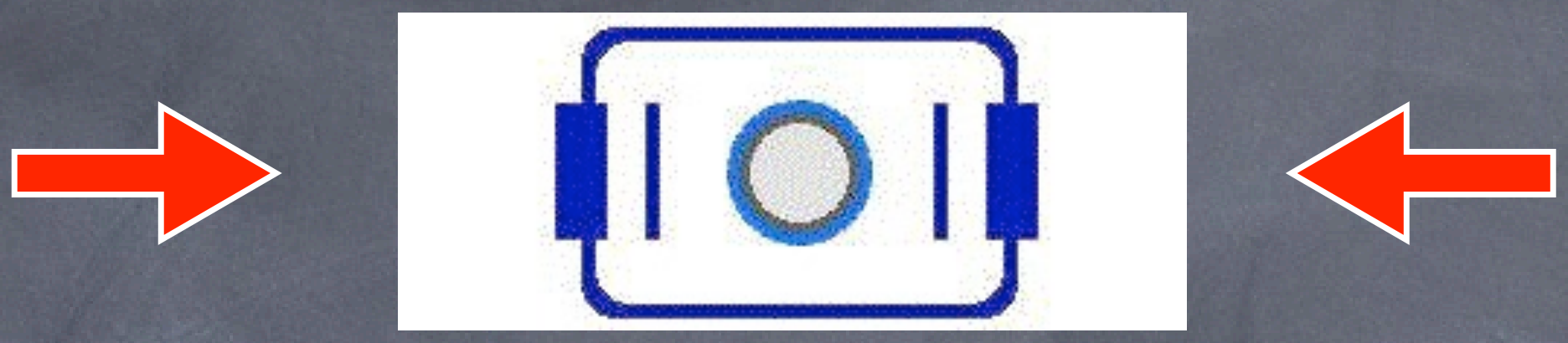

- Not really "confinement" at all

- Laser or ion beam to implode target

- Direct drive vs Indirect Drive

- Goal is to ignite DT fuel and burn significant fraction before capsule blows apart $\longrightarrow$

- ICF Ignition Experiments are being constructed in both U.S. and France 


\section{Significant Challenges Remain}

- Factor of 40 larger in energy than NOVA

- Can it be built and operated?

- Fabrication of capsules, laser glass, etc.

- Can we deliver the energy where we want and when we want? - shock timing

(อ)
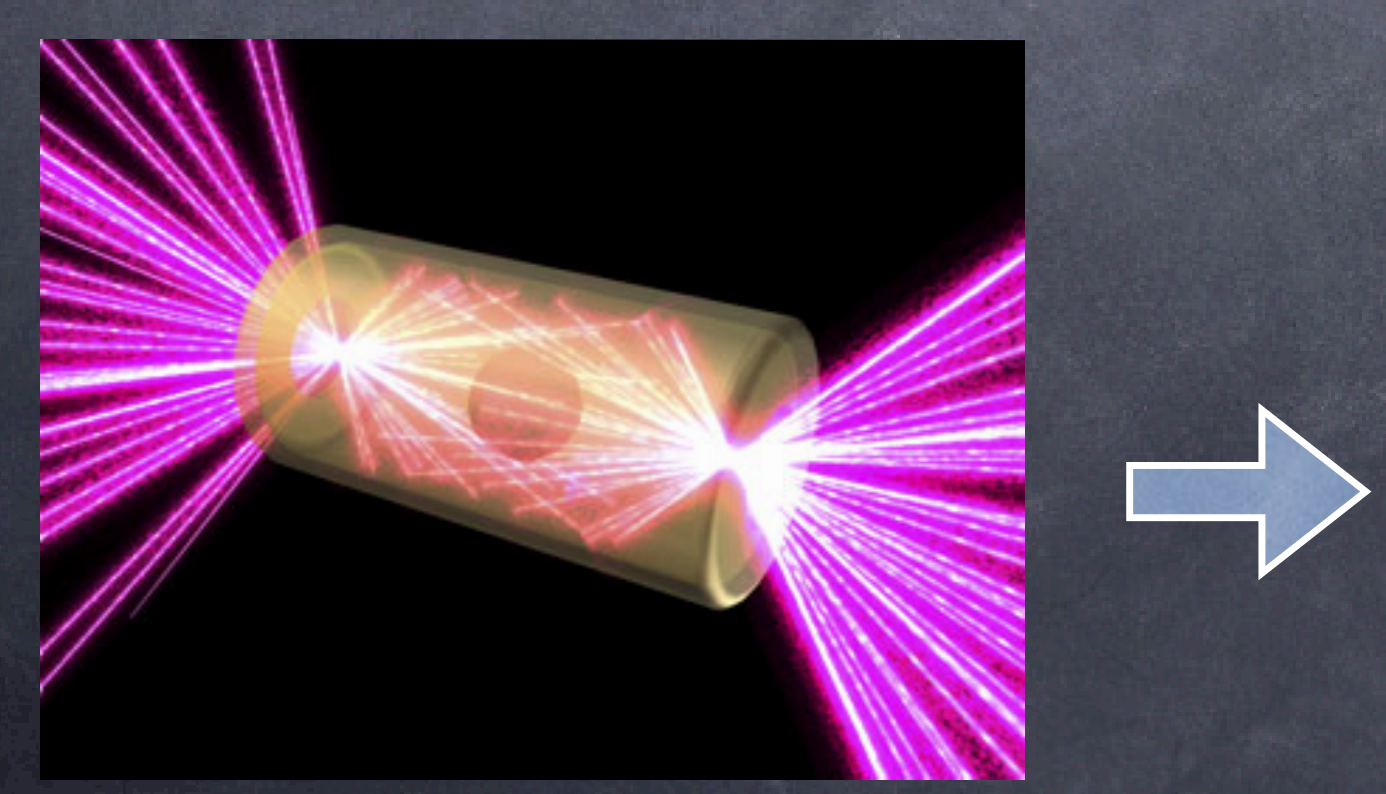

Plasma

$n_{e} \approx n_{c r i t}$

Gold Hohlraum 


\section{Summary}

- Particle-in-cell simulations are conceptually simple, but very powerful method for simulating kinetic plasmas

- Method scales very well on new petascale computers and this is permitting a much wider range of 2D and 3D studies

- Good reference books on PIC simulations:

I. Birdsall \& Langdon - Plasma Physics vis Computer Simulation 2. Hockney \& Eastwood - Computer Simulation using Particles 

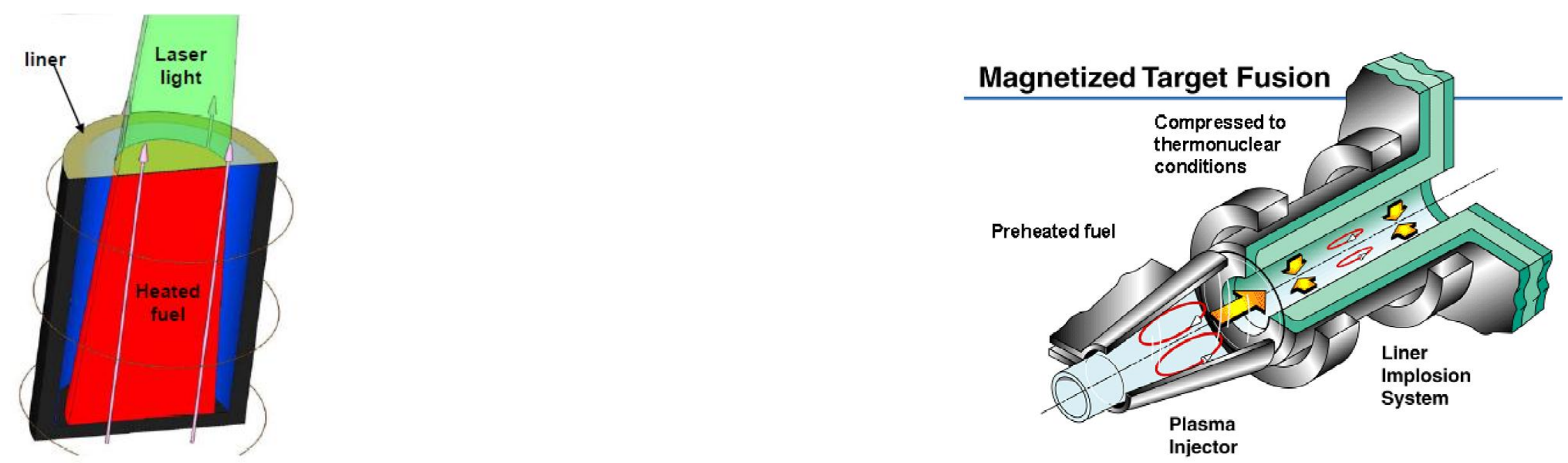

\section{Magneto-Inertial Fusion: Basic Principles}

Thomas J. Awe

Sandia National Laboratories

Presented to the LANL-P24 Plasma Physics Summer School

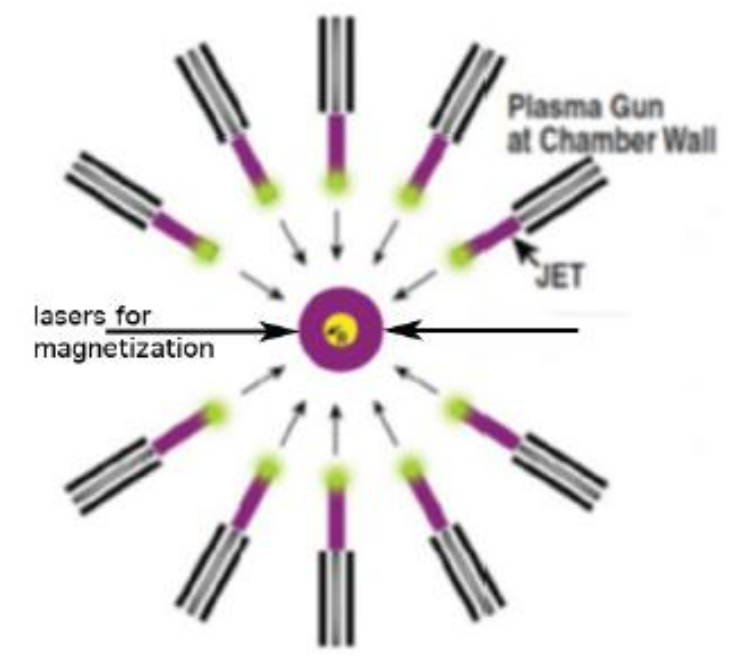

August 8, 2012

SAND Number: 2012-6567 P

Sandia National Laboratories

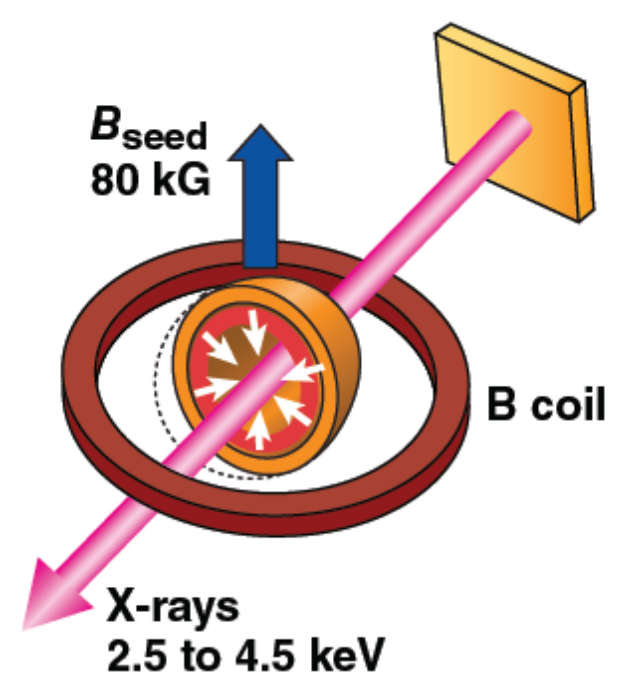




\section{Magneto-inertial fusion: Inertially confined magnetized fuel}
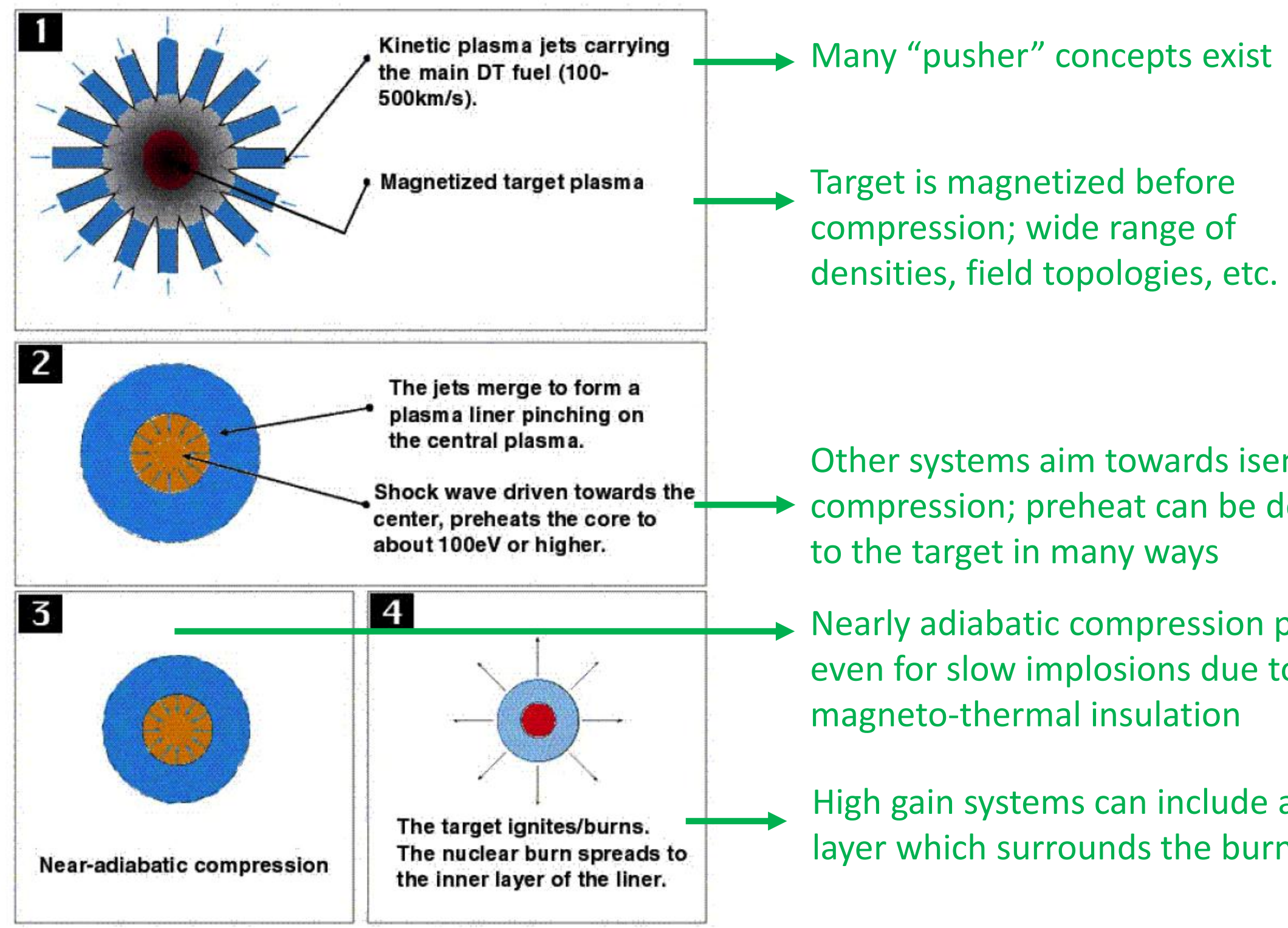

Other systems aim towards isentropic compression; preheat can be delivered to the target in many ways

Nearly adiabatic compression possible even for slow implosions due to magneto-thermal insulation

High gain systems can include a cold fuel layer which surrounds the burning fuel

From Y.C.F. Thio et al., Magnetized Target Fusion in a 


\section{The "ideal ignition temperature" is that temperature where internal heating exceeds losses}

\section{Internal heating}

$\mathrm{D}+\mathrm{T} \rightarrow \alpha(3.5 \mathrm{MeV})+\mathrm{n}(14.1 \mathrm{MeV})$

$\rightarrow$ Requires high areal density $(\rho R)$

$\rightarrow$ Inclusion of a magnetic field enables delivery of a larger fraction of the alpha energy to the fuel

\section{Loss Mechanisms}

Radiation loss $\rightarrow$ Dominated by (free-free) Bremsstrahlung for fully ionized plasma (what is opacity?)

Heat condution (electron or ion) $\rightarrow$ To surrounding cool fuel, or cool material pusher

$\rightarrow$ Heat conduction losses reduced if stagnation time is kept short

$\rightarrow$ Requires high density $\rightarrow$ high implosion velocity $\rightarrow$ expensive driver $\rightarrow O R$, heat conduction can be suppressed by inclusion of a strong magnetic field 


\section{Classical loss from radiation and thermal conduction}

\section{can be evaluated in a straightforward way}

$$
Q_{\text {loss }}=C_{R A D} n_{i}^{2} T^{1 / 2}-\nabla \bullet(K \nabla T)
$$

Both radiation loss and fusion rates depend on $n^{2}$, thus temperature alone dictates the energy balance. The fusion rate exceeds the radiation rate for $T>3 \mathrm{keV}$

$$
Q_{T C} \approx-\frac{1}{V} \int \nabla \cdot(K \nabla T) d V=-\frac{1}{V} \oint_{S} K \nabla T \cdot d \bar{S} \approx-\frac{S}{V} K \nabla T \approx \frac{K T}{\gamma \alpha \mathrm{a}^{2}}(11)
$$

where $V=\varepsilon \mathrm{a}^{3}, \quad \frac{V}{S}=\gamma \mathrm{a}, \nabla T \approx-\frac{T}{\alpha \mathrm{a}}$, a is a characteristic dimension, $V$ the plasma volume, $S$ the plasma surface area, $\varepsilon$ and $\gamma$ are geometric quantities (for example, for spheres, $\varepsilon=4 \pi / 3$ and $\gamma=1 / 3$ ), and $\alpha$ is a temperature-gradient scale factor that, as described later, depends on the form of $K$. 


\section{Magnetized fuel: Reduced cross-field thermal conductivity}

$\rightarrow$ Electrons gyrate around field lines: thermal conductivity is reduced perpendicular to field lines

$\rightarrow$ Hall parameter: $\chi_{\mathrm{e}}=\omega_{\mathrm{ce}} \tau_{\mathrm{ei}}$ indicates the degree of magneto-thermal insulation

$\rightarrow$ Thermal insulation when $\chi_{\mathrm{e}}=\omega_{\mathrm{ce}} \tau_{\mathrm{ei}}>1 \rightarrow \tau_{\mathrm{ei}}>1 / \omega_{\mathrm{ce}} \rightarrow \tau_{\mathrm{ei}}>\tau_{\mathrm{ce}}$

$\rightarrow$ implies time between collisions exceeds time for full gyration, so electrons stay "frozen" to field line

$\rightarrow$ Electron thermal conductivity written in terms of the Hall parameter is:

$K_{c e}=\frac{3.16 n k T}{m_{e}} \tau_{e}\left[\frac{1+0.39 \chi_{e}^{2}}{1+3.9 \chi_{e}^{2}+0.26 \chi_{e}^{4}}\right]$

$\frac{Q}{L}=C_{1} T^{7 / 2}\left[\frac{1+0.39 C_{2}^{2} T^{3}(B / \rho)^{2}}{1+3.9 C_{2}^{2} T^{3}(B / \rho)^{2}+0.26 C_{2}^{4} T^{6}(B / \rho)^{4}}\right] \quad[W / \mathrm{cm}]$

So, for large $\mathrm{B} / \rho \ldots \frac{Q}{L} \propto T^{1 / 2}\left(\frac{\rho}{B}\right)^{2} \quad$ And for $\mathrm{B}=0 \ldots \frac{Q}{L} \propto T^{7 / 2}$ 


\section{Classical loss from radiation and thermal conduction}

\section{can be evaluated in a straightforward way}

$$
Q_{\text {loss }}=C_{R A D} n_{i}^{2} T^{1 / 2}-\nabla \bullet(K \nabla T)
$$

Both radiation loss and fusion rates depend on $n^{2}$, thus temperature alone dictates the energy balance. The fusion rate exceeds the radiation rate for $T>3 \mathrm{keV}$

$$
Q_{T C} \approx-\frac{1}{V} \int \nabla \cdot(K \nabla T) d V=-\frac{1}{V} \oint_{S} K \nabla T \cdot d \bar{S} \approx-\frac{S}{V} K \nabla T \approx \frac{K T}{\gamma \alpha \mathrm{a}^{2}}(11)
$$

where $V=\varepsilon \mathrm{a}^{3}, \quad \frac{V}{S}=\gamma \mathrm{a}, \nabla T \approx-\frac{T}{\alpha \mathrm{a}}$, a is a characteristic dimension, $V$ the plasma volume, $S$ the plasma surface area, $\varepsilon$ and $\gamma$ are geometric quantities (for example, for spheres, $\varepsilon=4 \pi / 3$ and $\gamma=1 / 3$ ), and $\alpha$ is a temperature-gradient scale factor that, as described later, depends on the form of $K$. 


\section{Considering losses in simple geometric terms allows estimates of the required fuel volume for energy gain}

$$
Q_{T C} \approx \frac{K T}{\gamma \alpha \mathrm{a}^{2}} \longrightarrow \mathrm{a}^{2}=\frac{K T}{\gamma \alpha} \frac{1}{\phi Q_{D T}-Q_{R A D}} \quad \text { where } Q_{\text {loss }}=\frac{d E_{\text {loss }}}{d t}=\phi Q_{F U S}
$$

Once the minimum size required to operate at or below a specified value of $\phi$ for a specified density-temperature (and magnetic field) is determined, important physical parameters such as the minimum fuel mass $M$ and the minimum plasma thermal energy $E_{P L A S}$ can be determined:

$$
M=n_{i}\left(m_{i}+m_{e}\right) \varepsilon \mathrm{a}^{3}, \quad E_{P L A S}=3 n_{i} T \varepsilon \mathrm{a}^{3} .
$$

The minimum required heating rate to sustain the plasma, and the corresponding heating intensity, can also be determined:

$$
P_{H E A T}=Q_{T C}+Q_{R A D}, \quad I_{H E A T}=\frac{P_{H E A T}}{S} .
$$

From Section IV of Lindemuth \& Siemon, 


\section{The minimum system size is excessively large unless the density is very large}

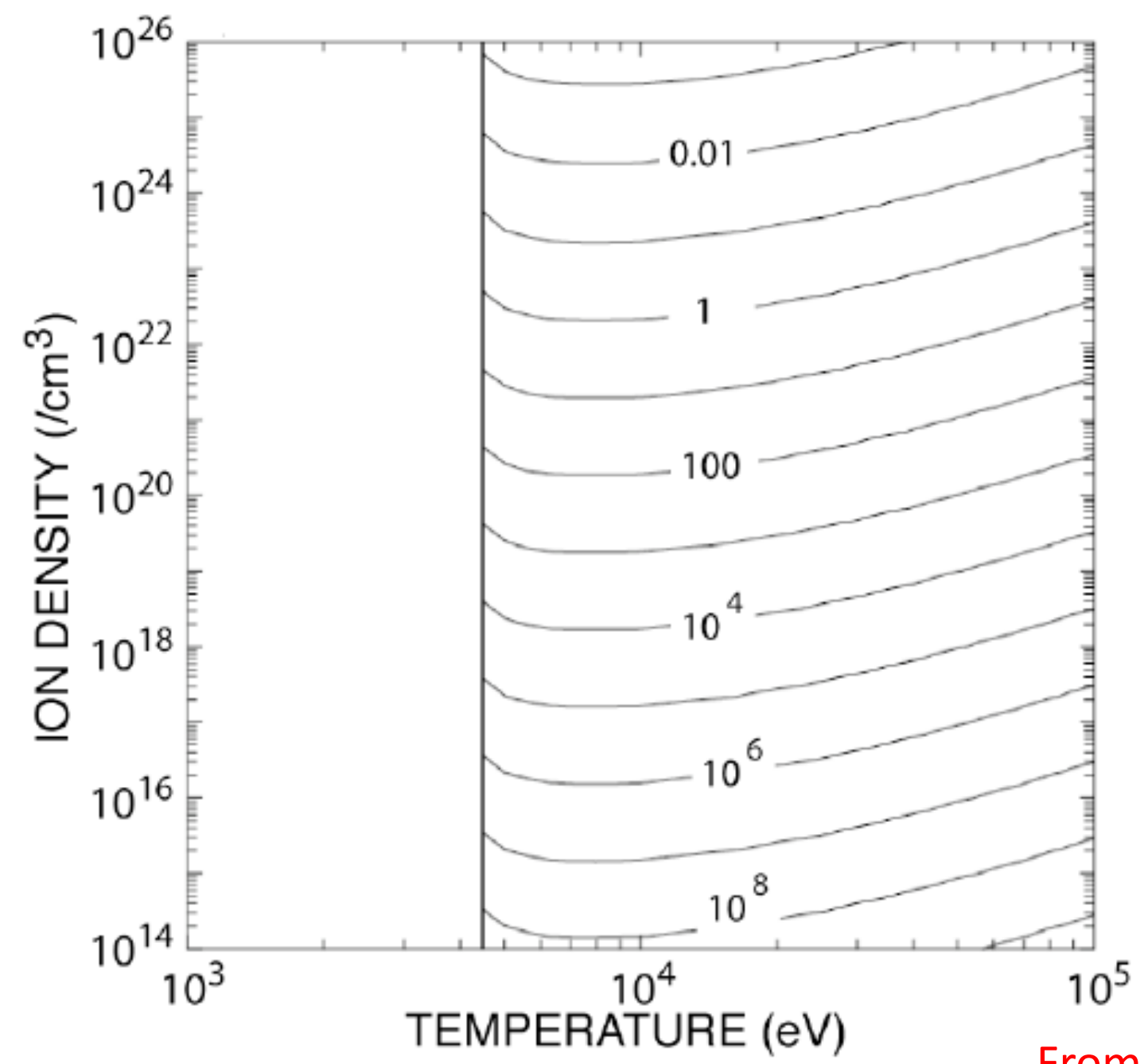

The minimum system size $(\mathrm{cm})$ for unmagnetized fuel (spherical geometry) operating at $\phi=0.2$. 
Extreme density is also required to avoid impossibly high minimum energy and power

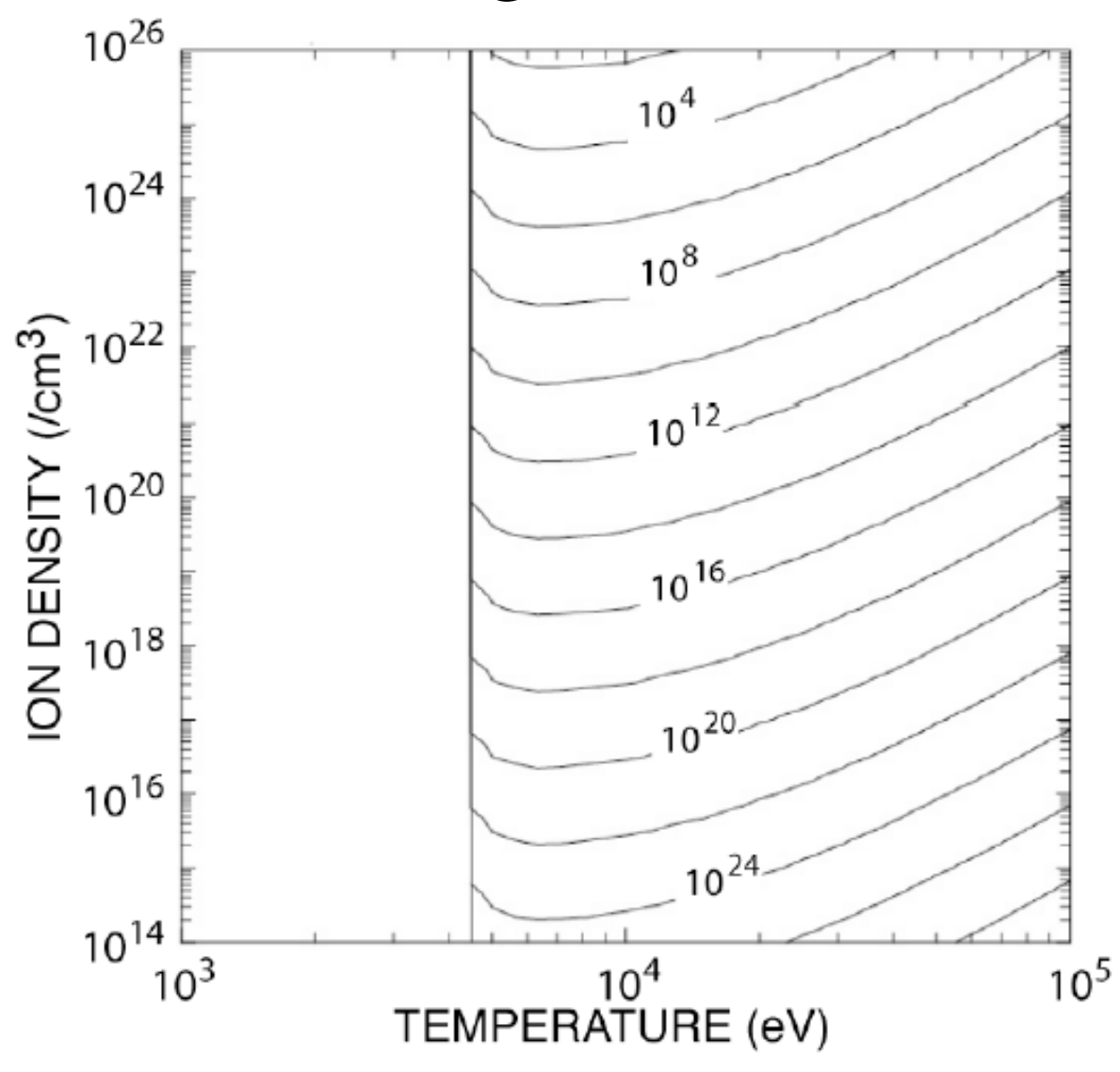

(a)

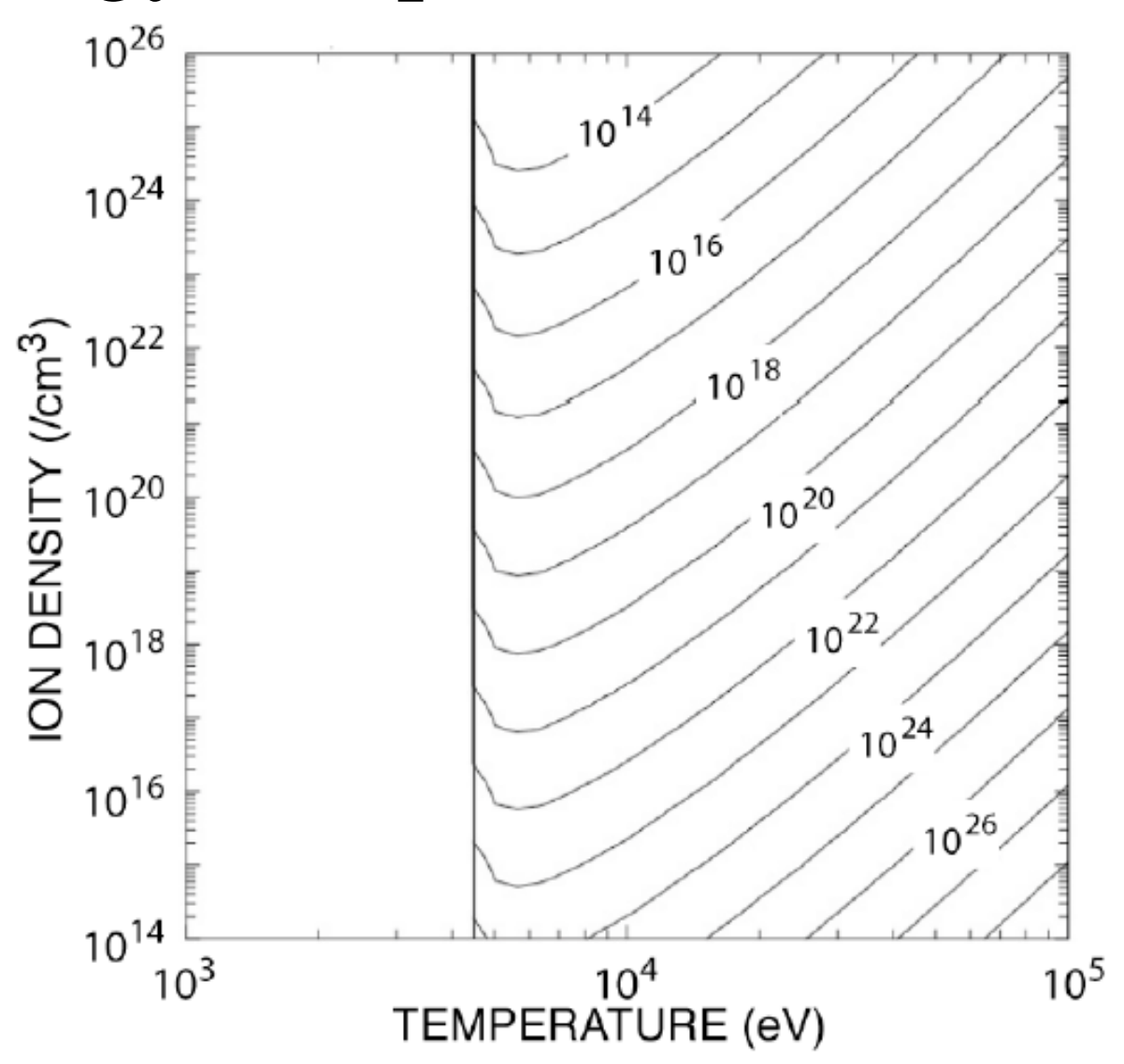

(b)

Figure 2. (a) The minimum energy (J), and (b) the minimum power (W) for unmagnetized fuel (spherical geometry) operating at $\phi \leq 0.2$.

From Section $V$ of Lindemuth \& Siemon, 


\section{For un-magnetized fuels, only extreme fuel}

\section{configurations are interesting for energy production}

High areal density, $\rho R \sim 3 \mathrm{~g} / \mathrm{cm}^{2}$ is required so that:

$\rightarrow$ Alphas deposit their energy in the fuel (self heating)

$\rightarrow$ The fractional burn up is high

See Atzeni \& Meyer-

Ter-Vehn, Ch2

\section{BUT...}

The fuel mass must be kept rather small $(<10 \mathrm{mg}$ ) since, for obvious practical reasons, the energy released per fusion event must be kept to a few GJ

AND...

Mass, $M=\rho V=\rho(4 / 3) \pi R^{3}=(4 / 3) \pi(\rho R)^{3} / \rho^{2}$

SO...

For fixed/minimum $\rho R$ (for reasons above), to accommodate the small mass requirement, $\rho$ must be extremely large:

$\rho^{2}=(4 / 3) \pi(\rho R)^{3} / M \rightarrow \rho \sim 336 \mathrm{~g} / \mathrm{cm}^{3}$

\section{This is $\sim 1500$ times the density of cryogenic DT!!!}




\section{Inclusion of a strong magnetic field reduces the system size, energy and power requirements}

Cross field thermal conductivity is reduced by a factor proportional to $B^{-2}$

In the following plots, $\beta$ (ratio of plasma pressure to magnetic pressure is held equal to 1 , so the field strength changes as a function of $n, T$ as:

$$
B=2.8 \times 10^{-4}\left(n_{i} T_{k e V} / \beta\right)^{1 / 2}
$$

The minimum system

size $(\mathrm{cm})$ for magnetized fuel (spherical geometry) operating at $\phi<0.2$.

From Section VI of Lindemuth \& Siemon, Am. J. Phys., Vol 77, No. 5, May 2009

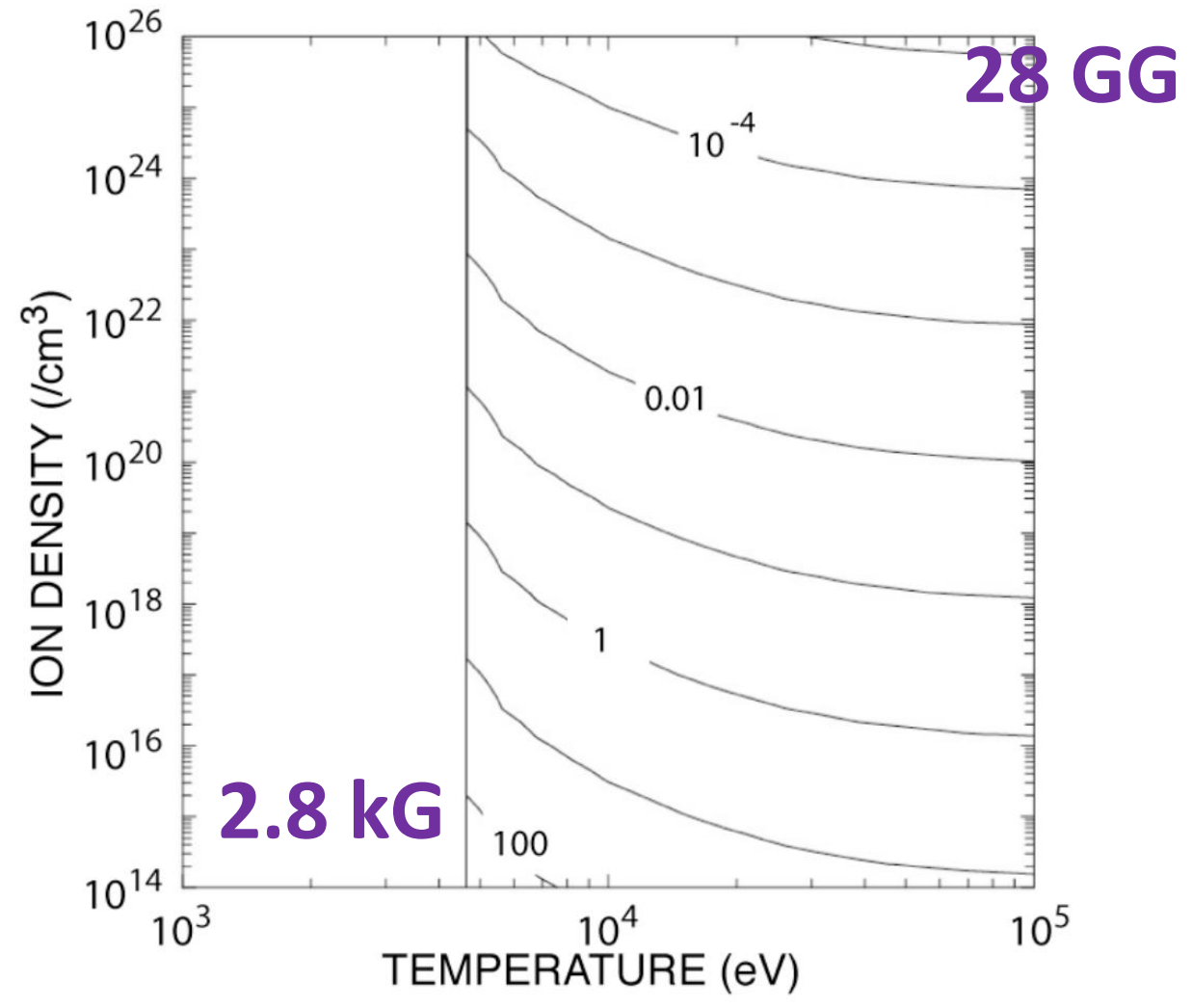




\section{Inclusion of a strong magnetic field reduces the}

system size, energy and power requirements

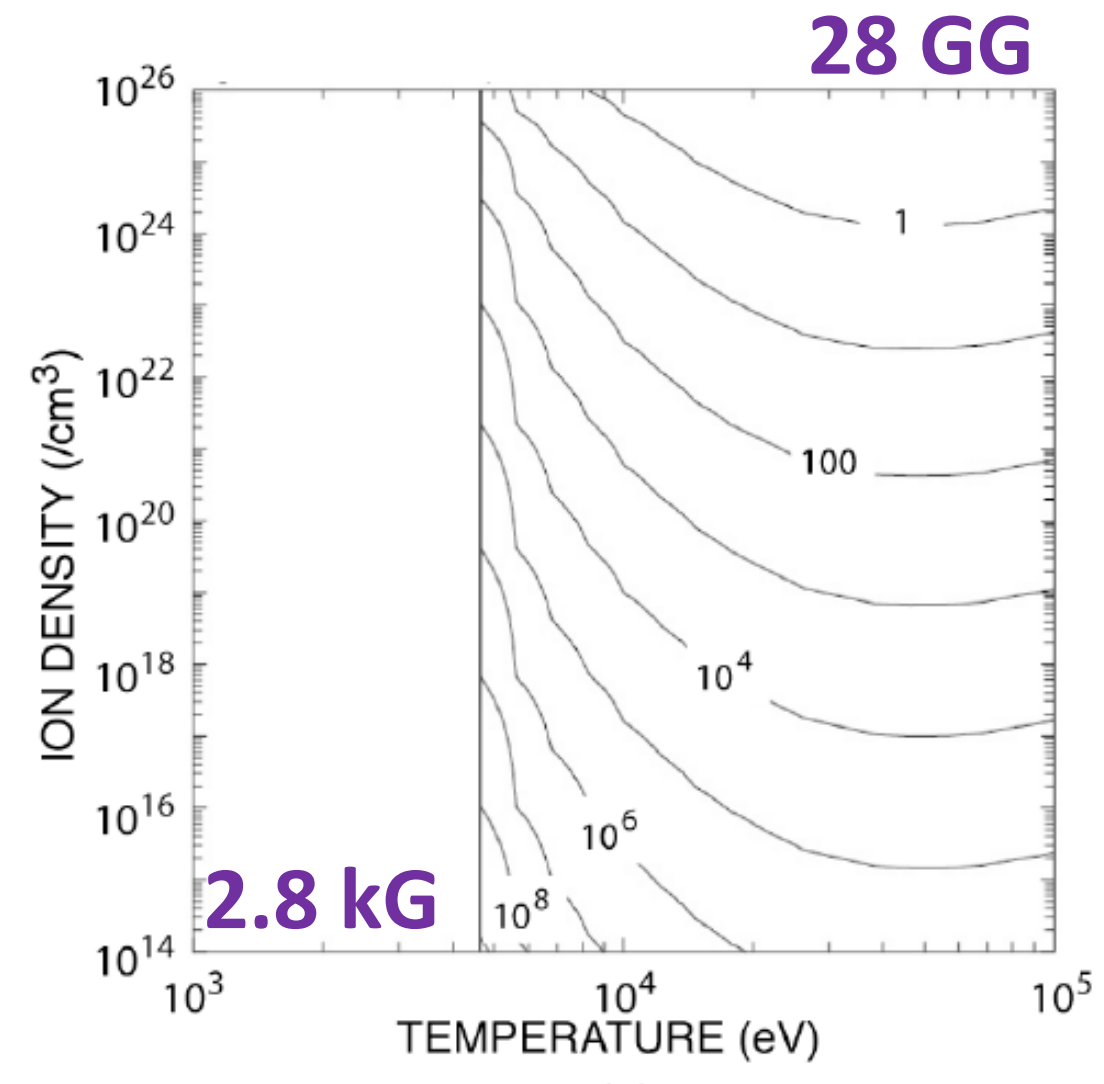

(a)

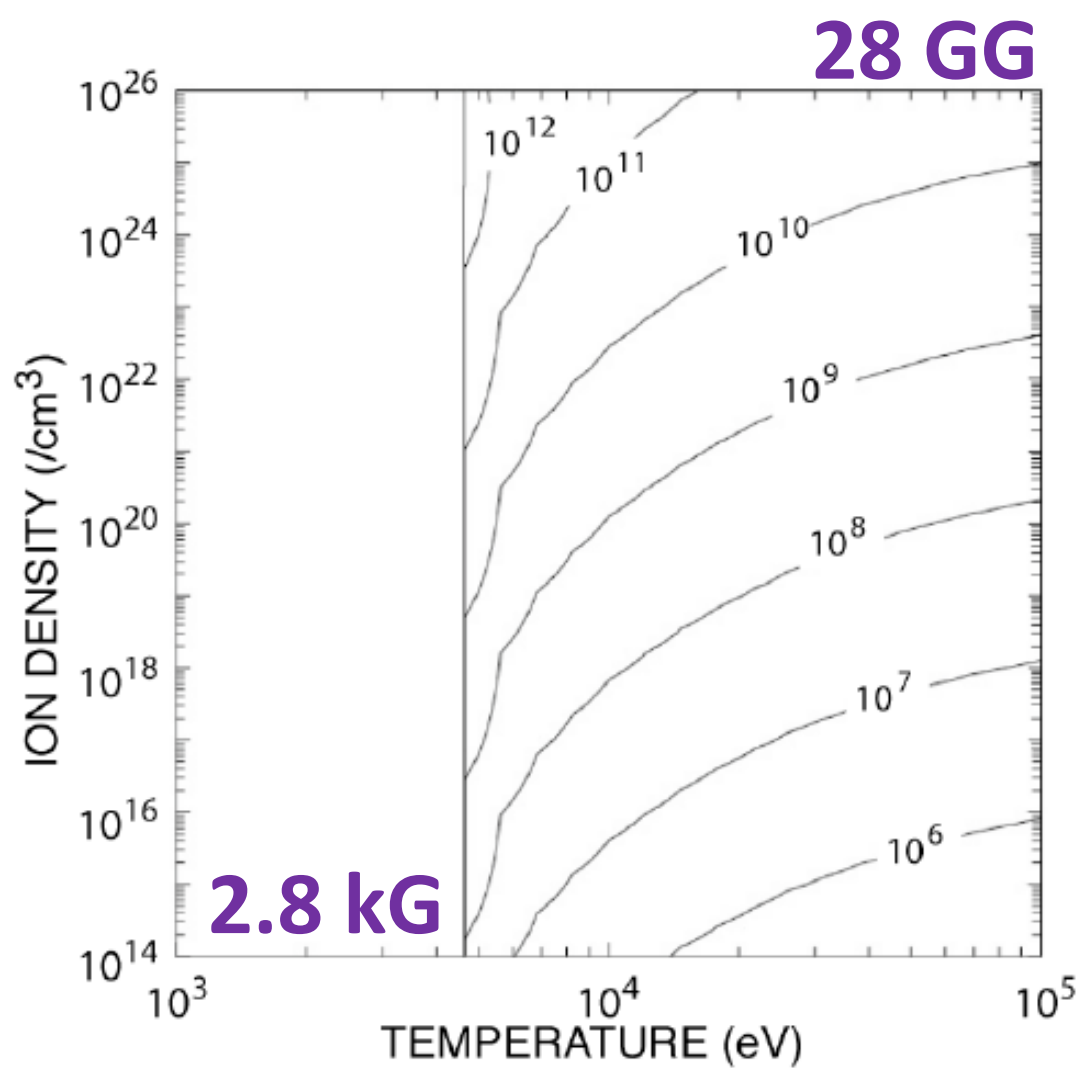

(b)

Figure 4. (a) The minimum energy (J), and (b) the minimum power (W) for magnetized fuel (toroidal geometry, $\beta=1$ ) operating at $\phi \leq 0.2$.

From Section VI of Lindemuth \& Siemon, 


\section{Introduction of a strong magnetic field into the fuel opens up the ignition parameter space}

Ignition conditions for magnetized target fusion in cylindrical geometry

M.M. Baskoa*, A.J. Kemp ${ }^{\text {b }}$, J. Meyer-ter-Vehn ${ }^{\mathrm{b}}$

Nuclear Fusion, Vol. 40, No. 1 (2000)

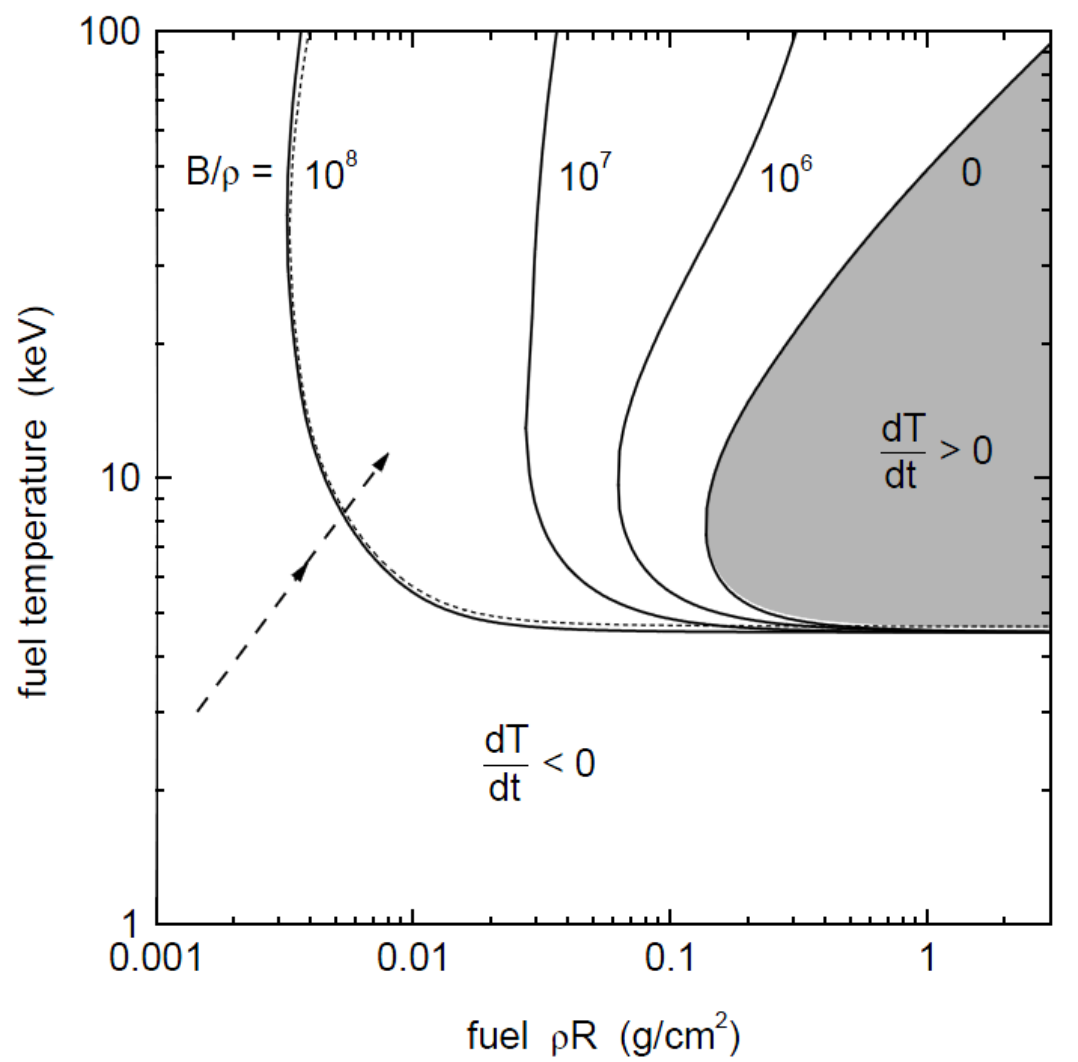

Recall, for large $\mathrm{B} / \rho$ :

$$
\frac{Q}{L} \propto T^{1 / 2}\left(\frac{\rho}{B}\right)^{2}
$$

Figure 3. Lindl-Widner diagram for magnetized DT cylinders at stagnation. Solid curves show a series of ignition boundaries in the $\rho R, T$ plane calculated for four fixed values of the parameter $B / \rho$, given near each curve in units of $\mathrm{G} \mathrm{cm}^{3} / \mathrm{g}$. The shaded area is the pure ICF ignition domain at $B=0$. The dotted curve illustrates the effect of synchrotron radiation losses at $\rho=1 \mathrm{~g} / \mathrm{cm}^{3}$, $B=10^{8} \mathrm{G}$. Dashed arrows indicate how the fuel states advance towards the ignition boundary in the process of a quasi-adiabatic implosion. 


\section{Embedded magnetic fields can be compressed to very}

high values with little effect on implosion dynamics Radial Compression of $B_{z}$

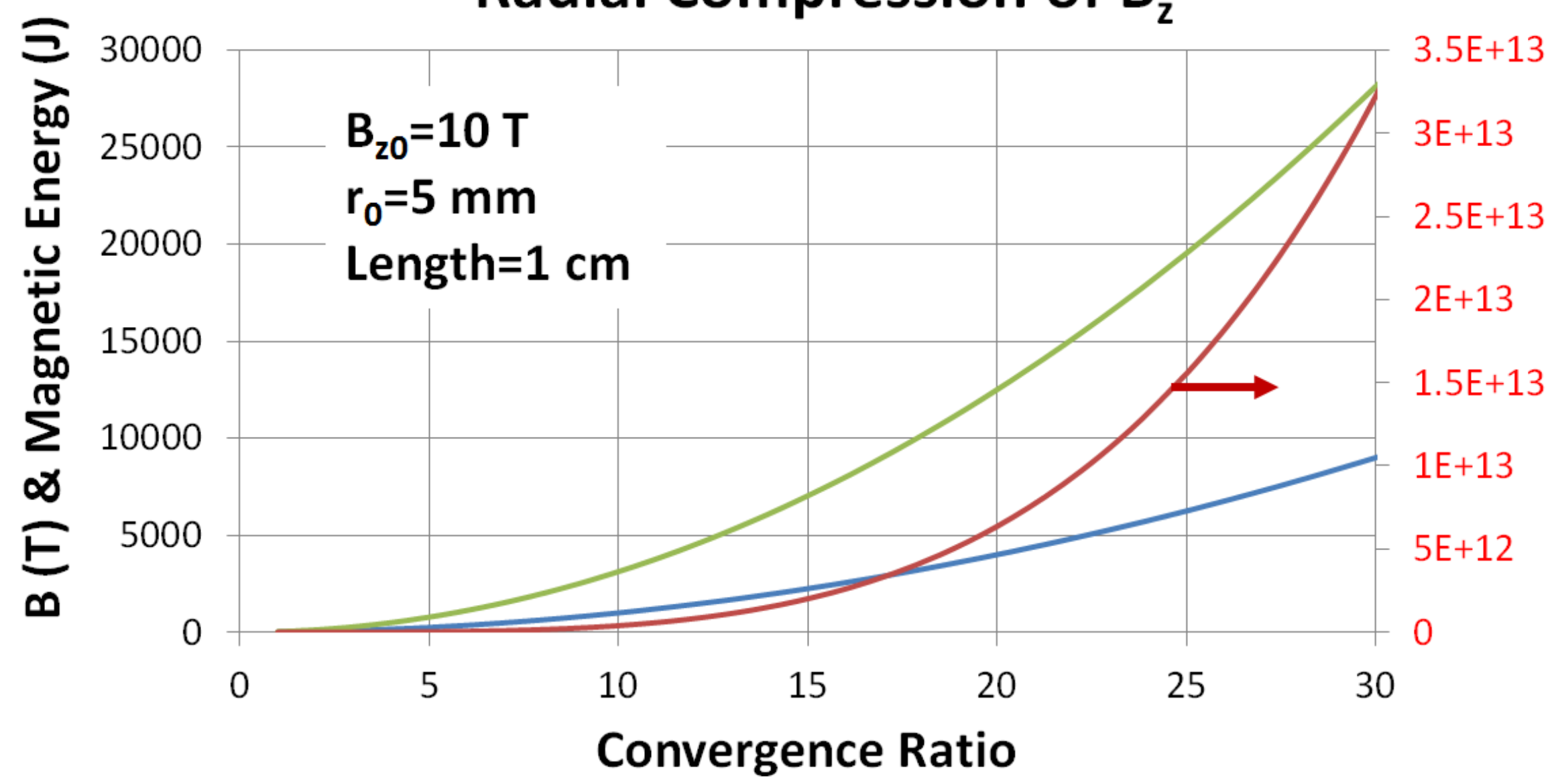

-B -Field Energy —Magnetic Pressure ***Magnetic pressure for an I=25 MA driver on a $\mathbf{3 0 0}$ micron radius liner is $10^{14} \mathrm{~Pa}$ 
The evolution of the liner inner surface is a major unknown physics issue for MIF

- How \& when does liner inner surface change state?

- How well does liner confine magnetic flux?

- How big does RT grow during liner deceleration?

- How much high-Z mixing with DT fuel?

- How accurate is numerical modeling?

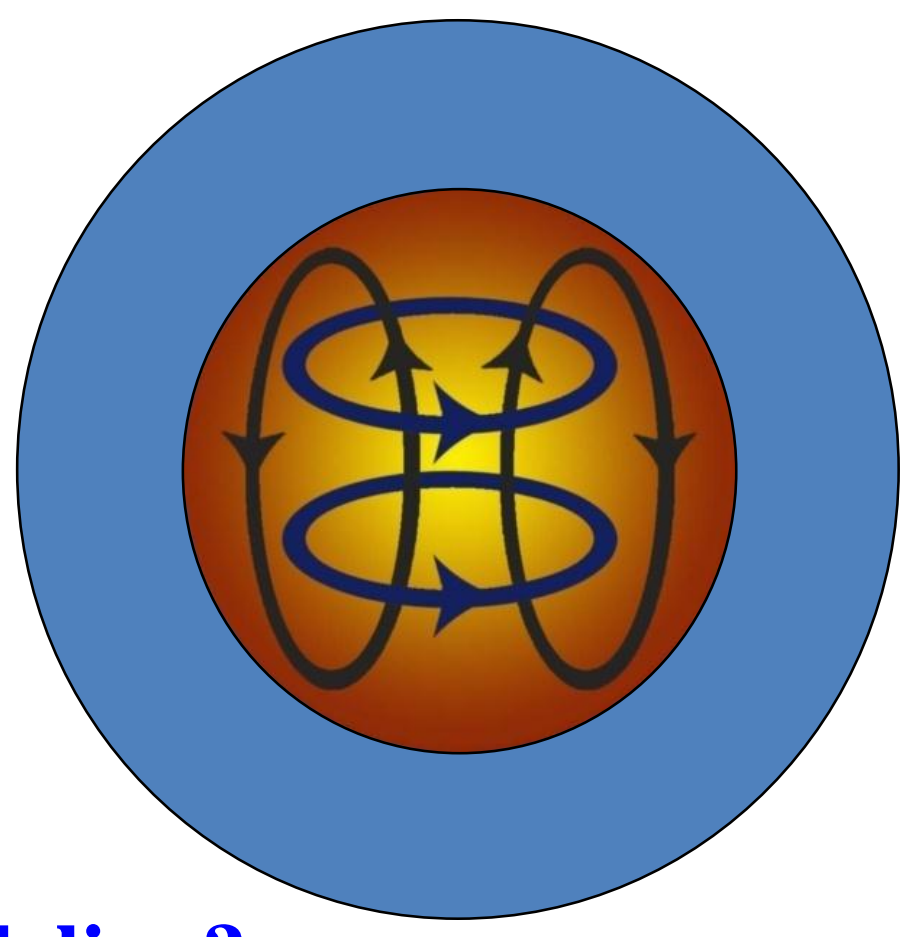




\section{A variety of magneto-inertial fusion efforts leverage the apparent advantages of magnetized fuel}

MIFEDS $\rightarrow$ laser (direct drive) ICF with magnetized target PLX $\rightarrow$ Plasma jets form spherical liner to compress magnetized target

FRCHX $\rightarrow$ FRC formed in theta coils - translated into and compressed by large imploding metallic liner MagLIF $\rightarrow$ Fast (100 ns) metallic liner compresses a preheated and dense fuel with an axial magnetic field

Other MIF related concepts: MAGO LINUS

General Fusion 


\section{Single-coil B field was used for fusion-enhancement measurements in spherical geometry}

FSC

- Single-coil provides stronger seed fields (80 kG), less interference with laser beam

- 40 beams ( $18 \mathrm{~kJ} / \mathbf{1} \mathrm{ns})$ were used for compression

- Implosion uniformity is diagnosed using $x$-ray $B L$ radiography

- nTOF diagnostic was used for $T_{i}$ and neutron-yield measurements

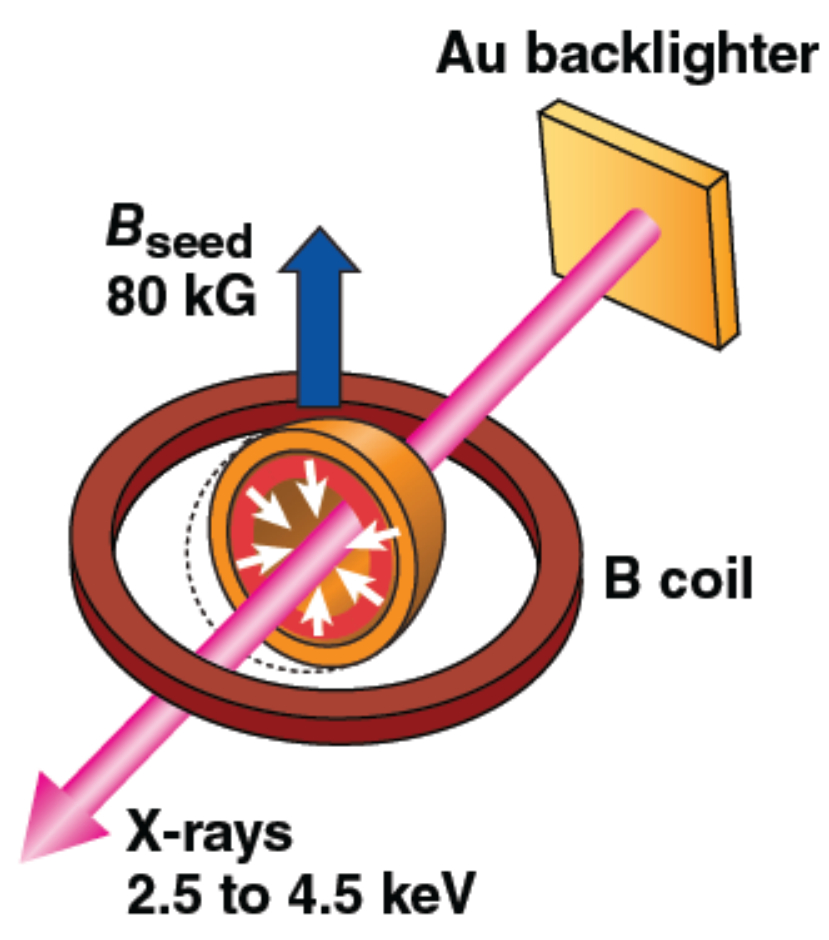




\section{The seed B field is created by a compact, self-contained magnetic field generator}

FSC

UR

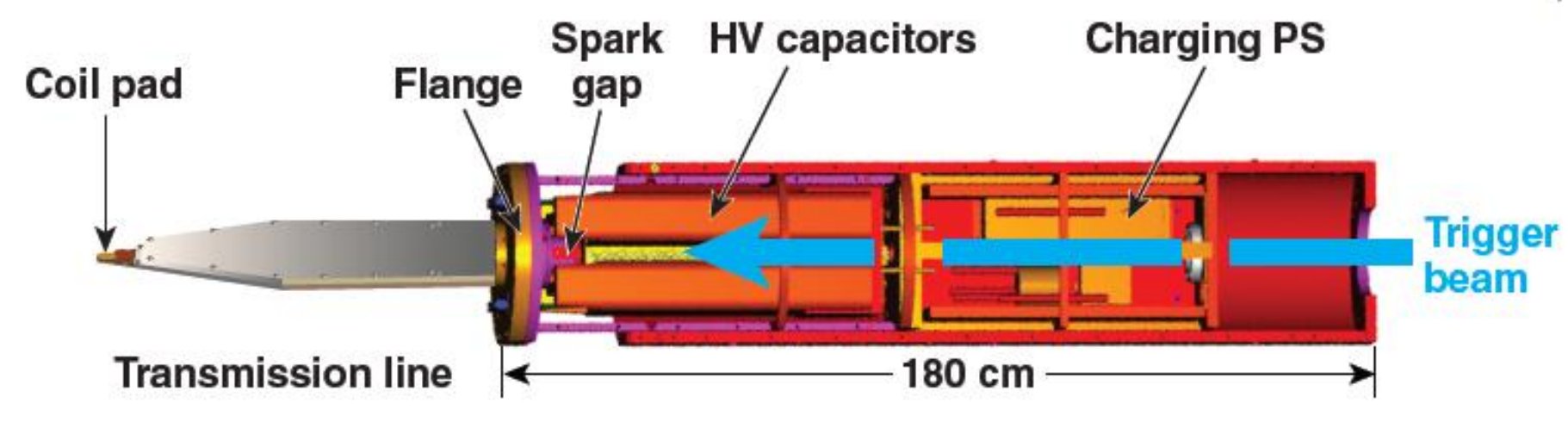

- MIFEDS-Magnetized Inertial Fusion Energy Delivery System

- Various coils were tested

- Seed fields up to $150 \mathrm{kG}$ can be obtained (depends on the coil size and geometry)

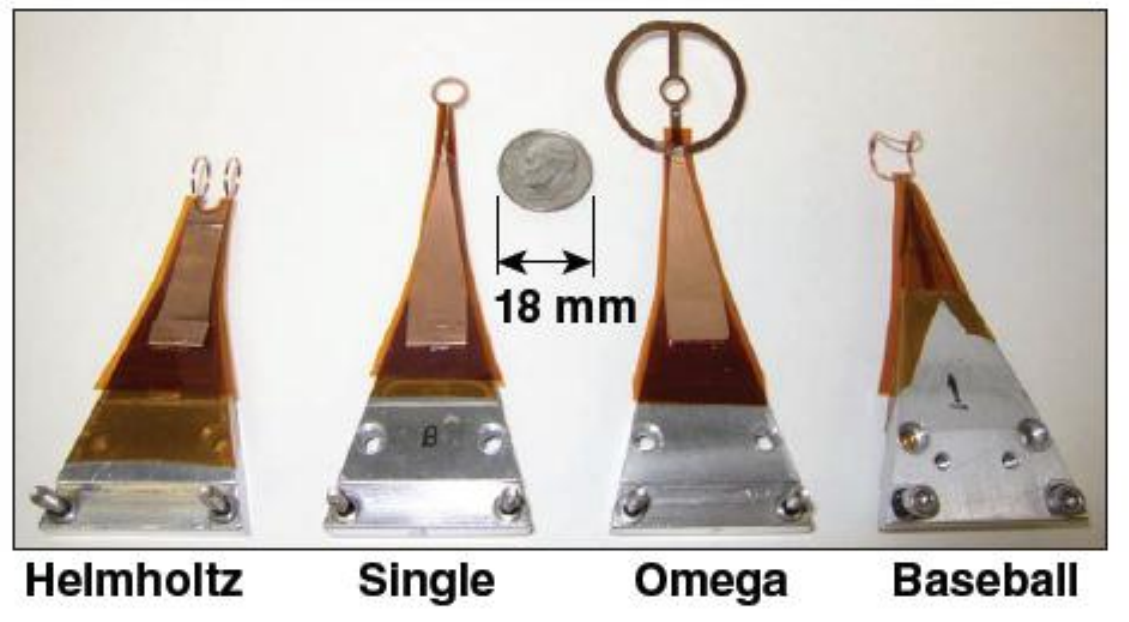

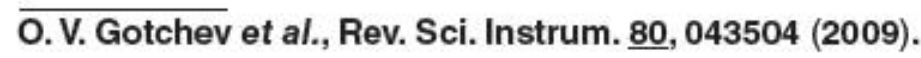


We observe a (30 \pm 12$) \%$ neutron-yield enhancement and $(15 \pm 4) \%$ ion-temperature increase for magnetized targets

\section{FSC}
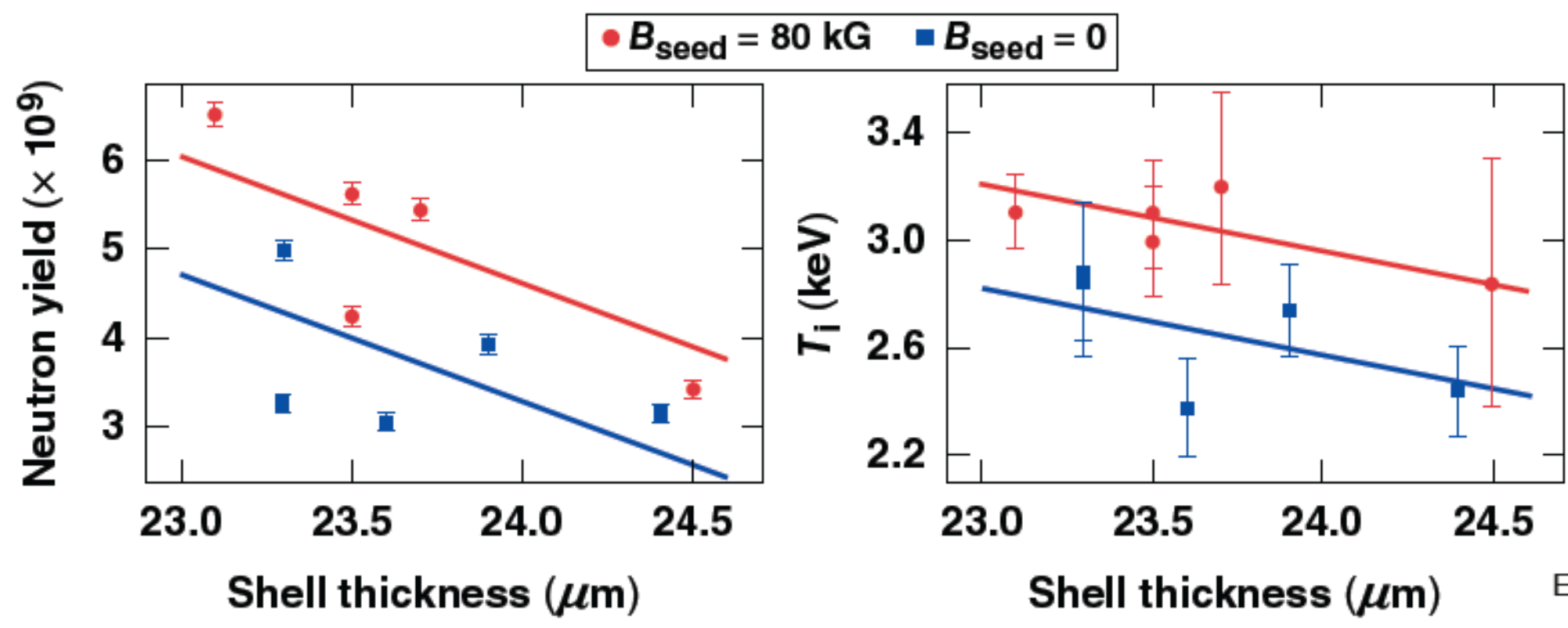

- Diagnostic-nTOF (neutron time-of flight)

- Fusion performance scales with shell thickness

Linear regression fit reveals clear enhancement of magnetized hot-spot performance. 
Motivation: Convergent plasma jets could be an attractive approach to inertial fusion energy (IFE) using magnetic fields
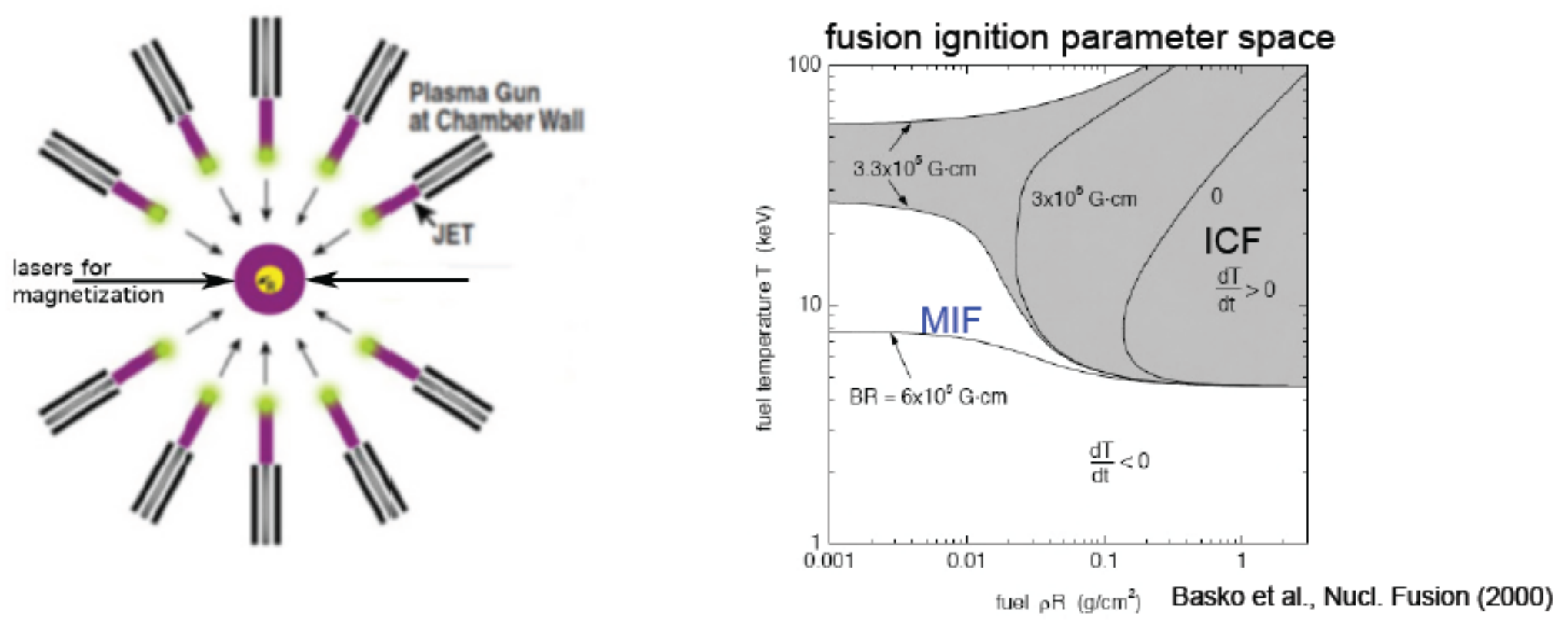

- Composite DT (yellow) and high-Z (purple) jets are imploded to $\rho r_{\mathrm{DT}} \sim 0.01 \mathrm{~g} / \mathrm{cm}^{2}$

- DT is magnetized via lasers just before peak compression

- Implosion speed $\sim 100 \mathrm{~km} / \mathrm{s}$

- Dwell time $\sim 1 \mu \mathrm{s}$

- Batch burn with $\sim \mathbf{1 0 \%}$ fuel burn-up 
First plasma 9/13/11: Single-jet experiments are underway
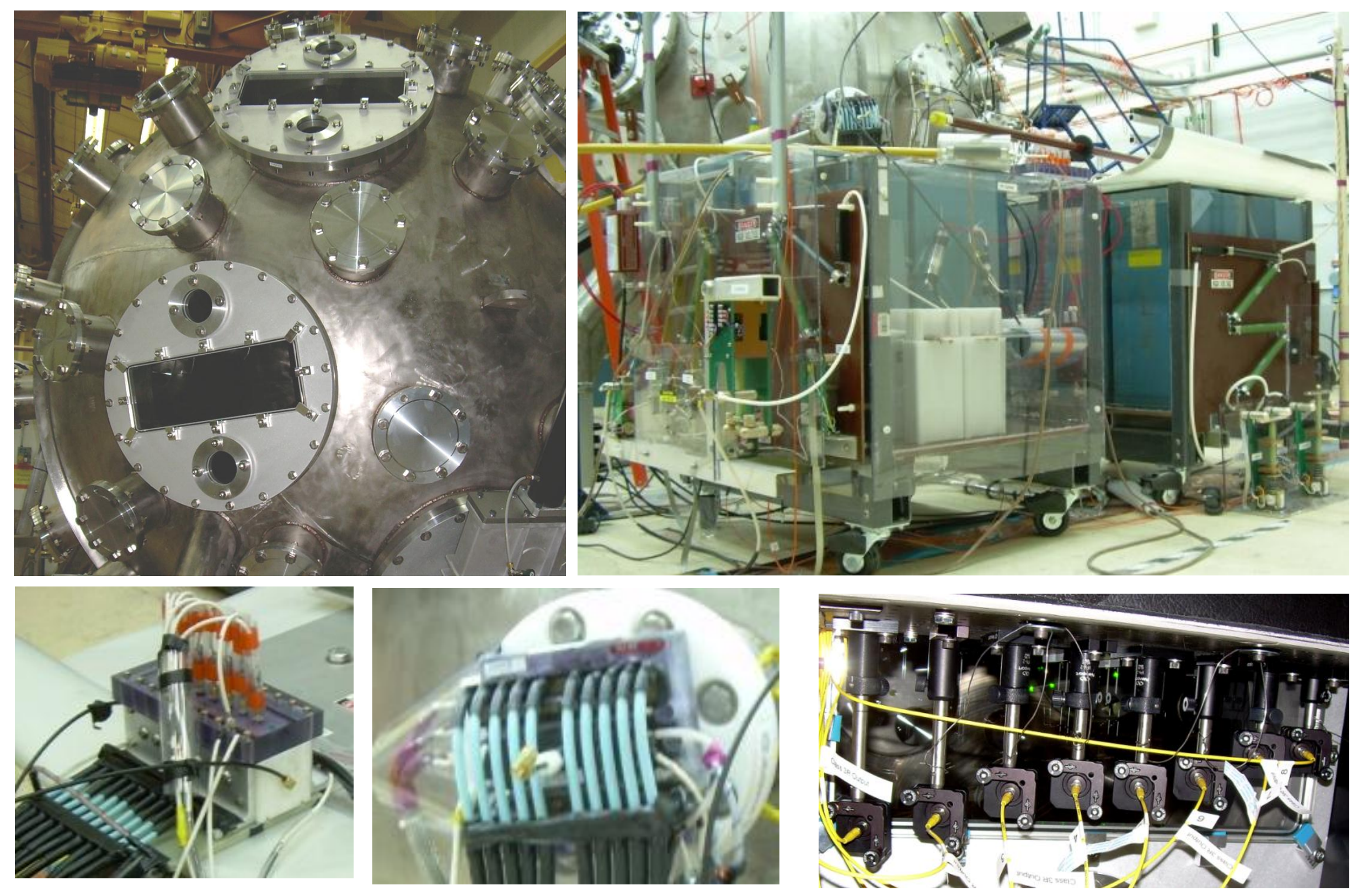


\section{Complex structure exists as the jet exits the gun bore and propagates down the nozzle}

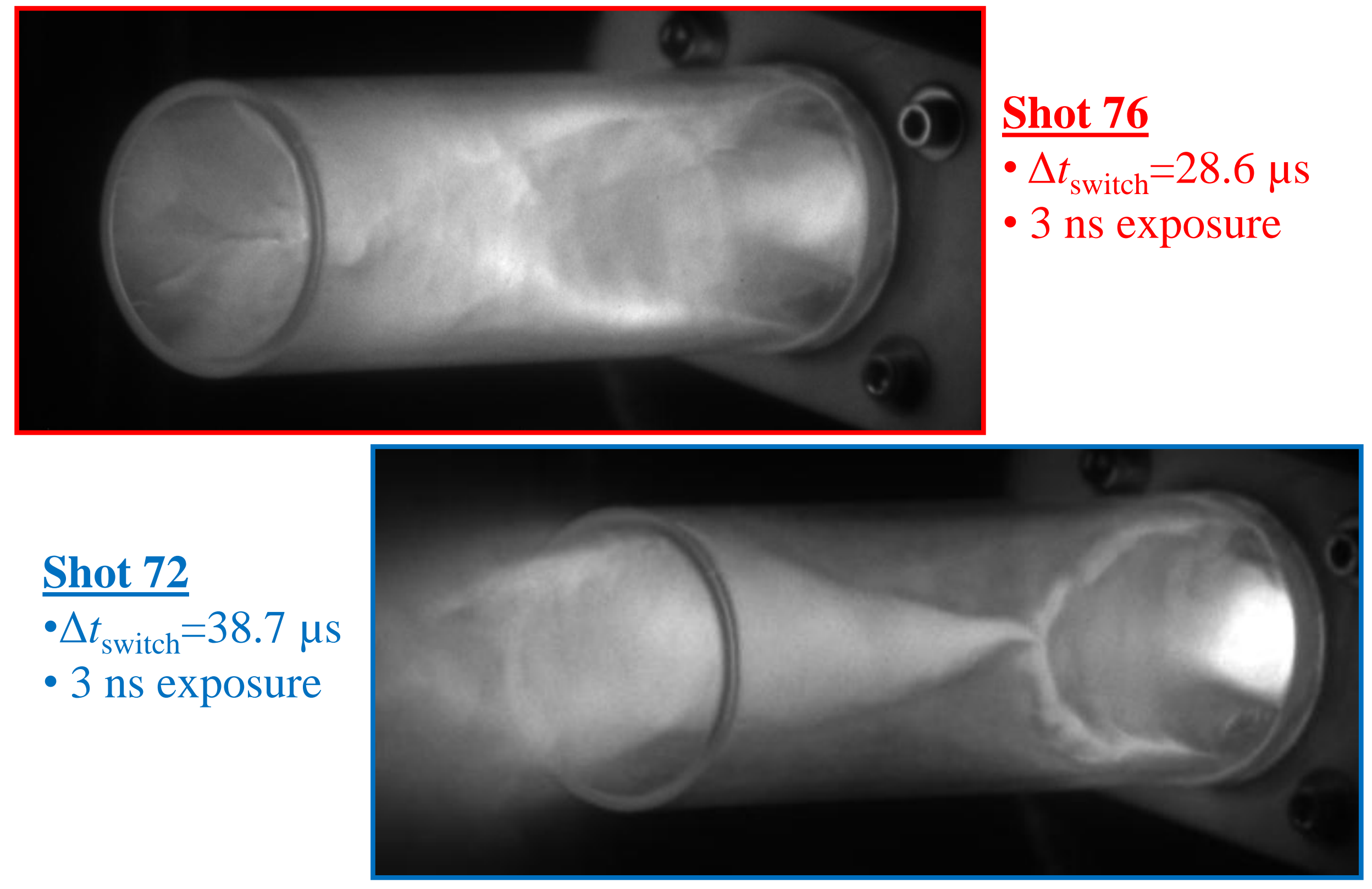


Fine structure in plasma jet persists long after leaving the nozzle $\rightarrow$ Can MHD explain complex plasma dynamics?
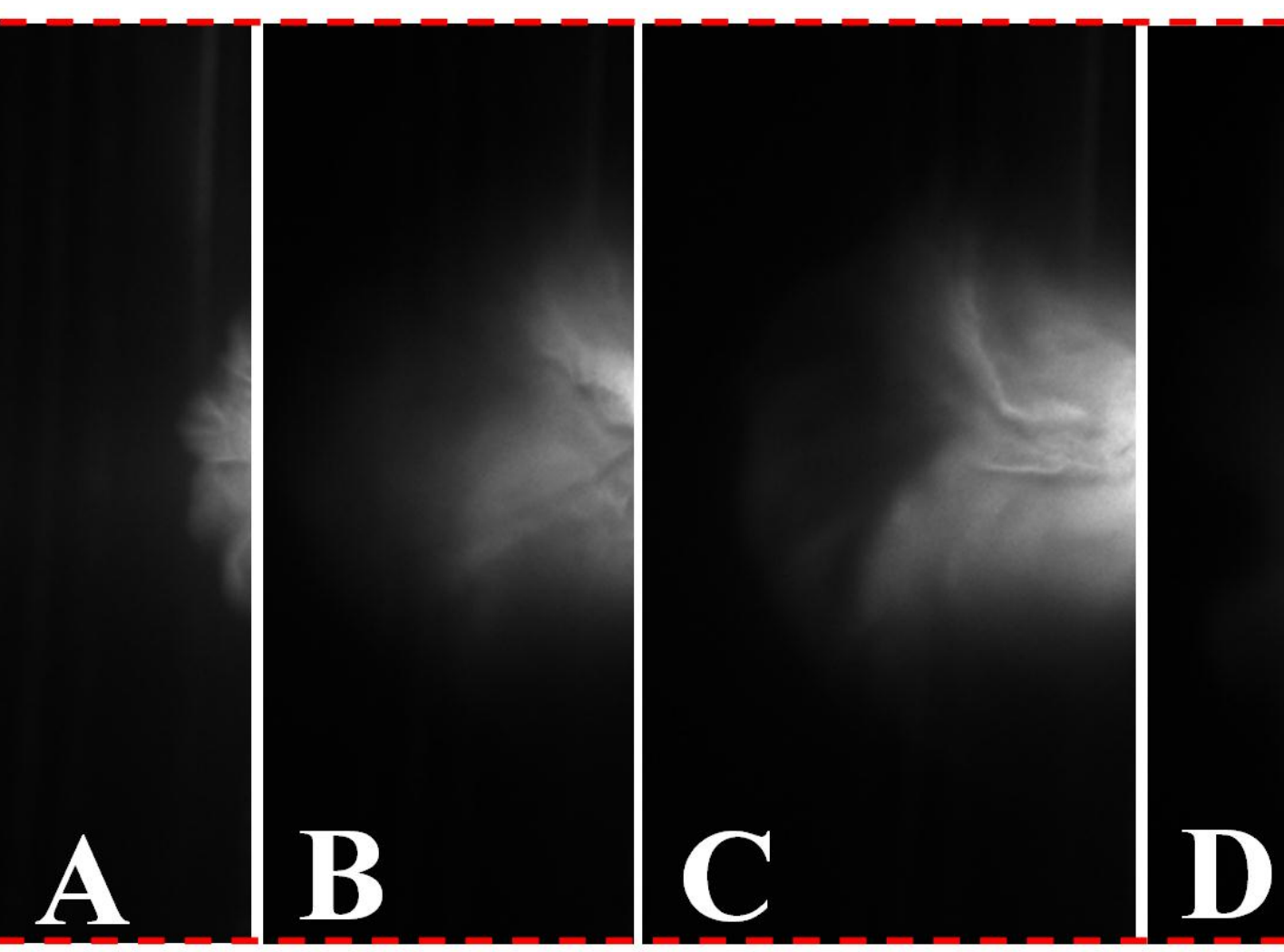

A: Shot 65 (shutter A), $29 \mu$ s after switch closure, $v_{\text {jet }} \sim 11 \mathrm{~km} / \mathrm{s}$ B: Shot 57 (shutter A), $29 \mu \mathrm{s}$ after switch closure, $v_{\text {jet }} \sim 19 \mathrm{~km} / \mathrm{s}$ C: Shot 59 (shutter A), $28.7 \mu \mathrm{s}$ after switch closure, $v_{\text {jet }} 21 \mathrm{~km} / \mathrm{s}$ D: Shot 65 (shutter A), $39 \mu \mathrm{s}$ after switch closure, $v_{\text {jet }} \sim 11 \mathrm{~km} / \mathrm{s}$ 


\section{Formation: LANL}

cusp

\section{Translation}

Compression mirror segmented

coils theta-pinch coil separatrix

@AFRL

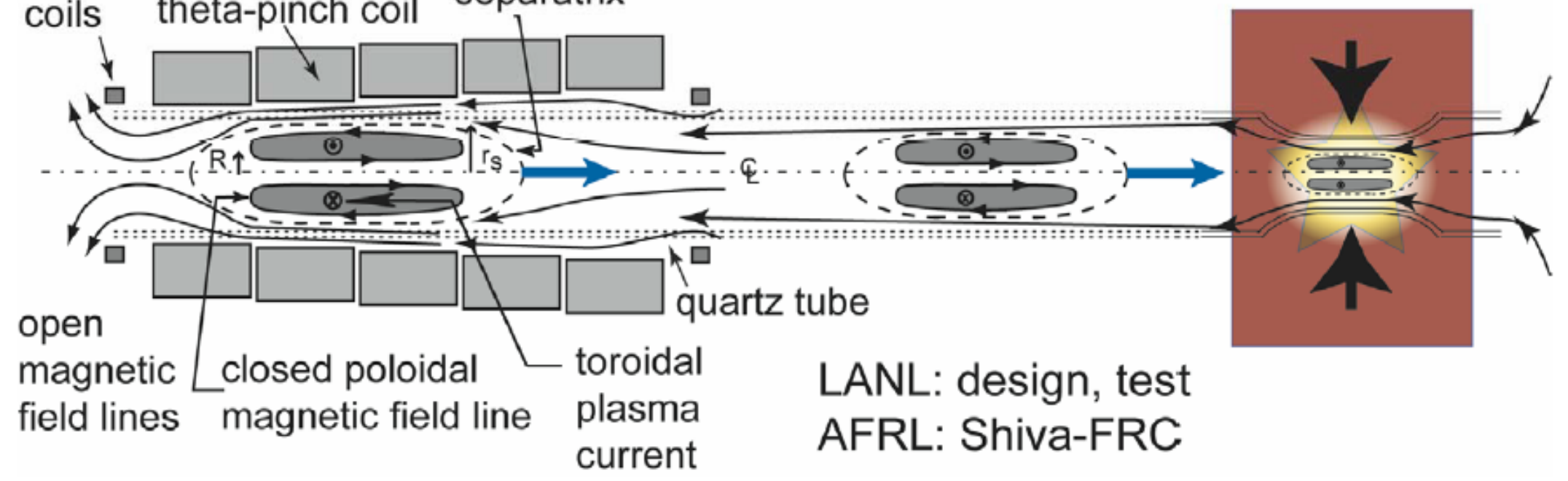

- Pulsed, high pressure approach to fusion

- Inertial + magnetic confinement

- Multi-keV fusion grade plasma 


\section{LANL's FRX-L Field Reversed Configuration (FRC) for target plasma development}

\section{Project/Concept}

Description: Magnetized Target Fusion. Develop a suitable plasma injector using a high density FRC

GOAL: To make the first physics demonstration of MTF by imploding a field reversed configuration plasma with a metal liner

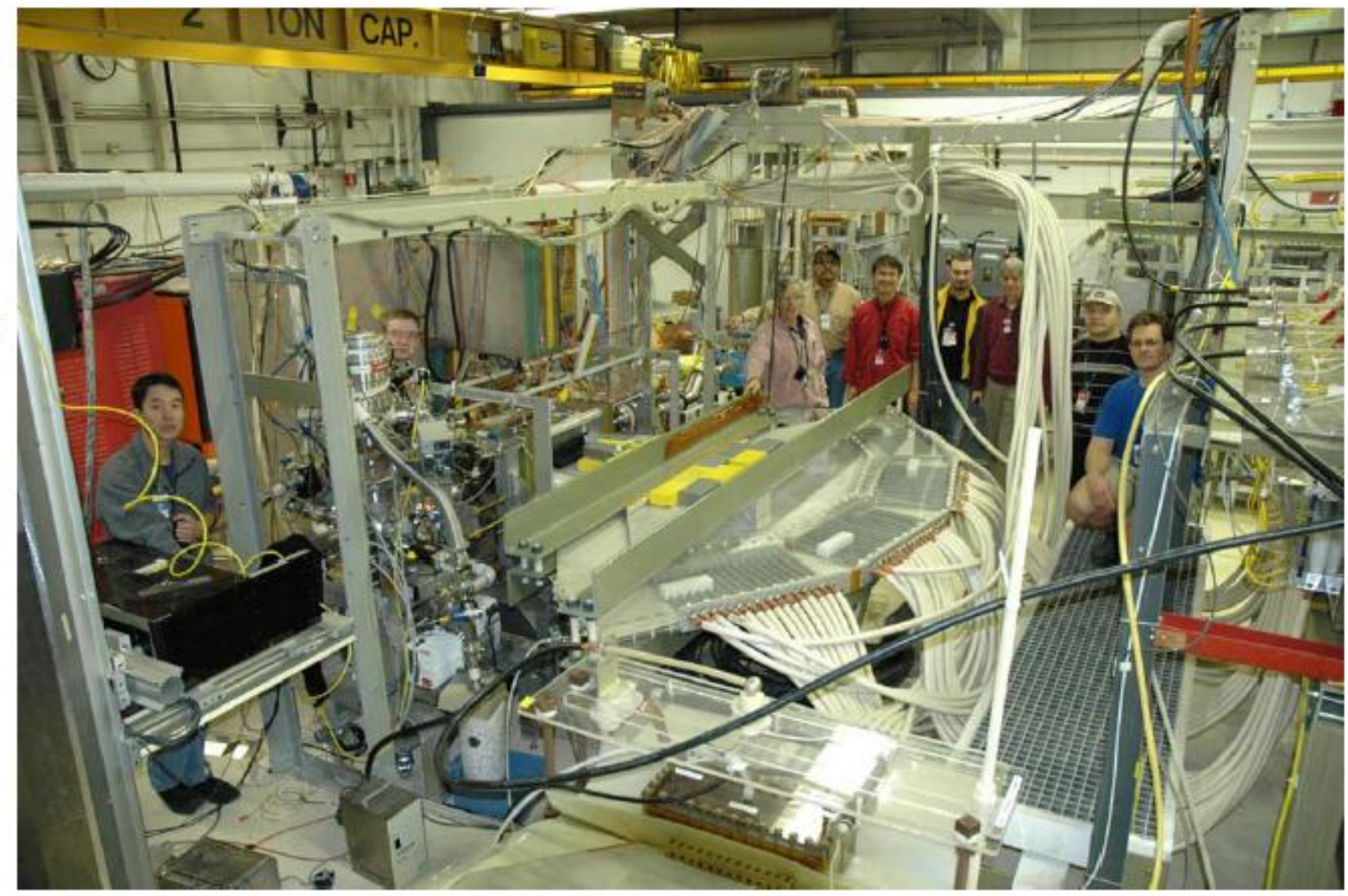

- The FRX-L experiment and team 


\section{Shiva Star Facility at AFRL}

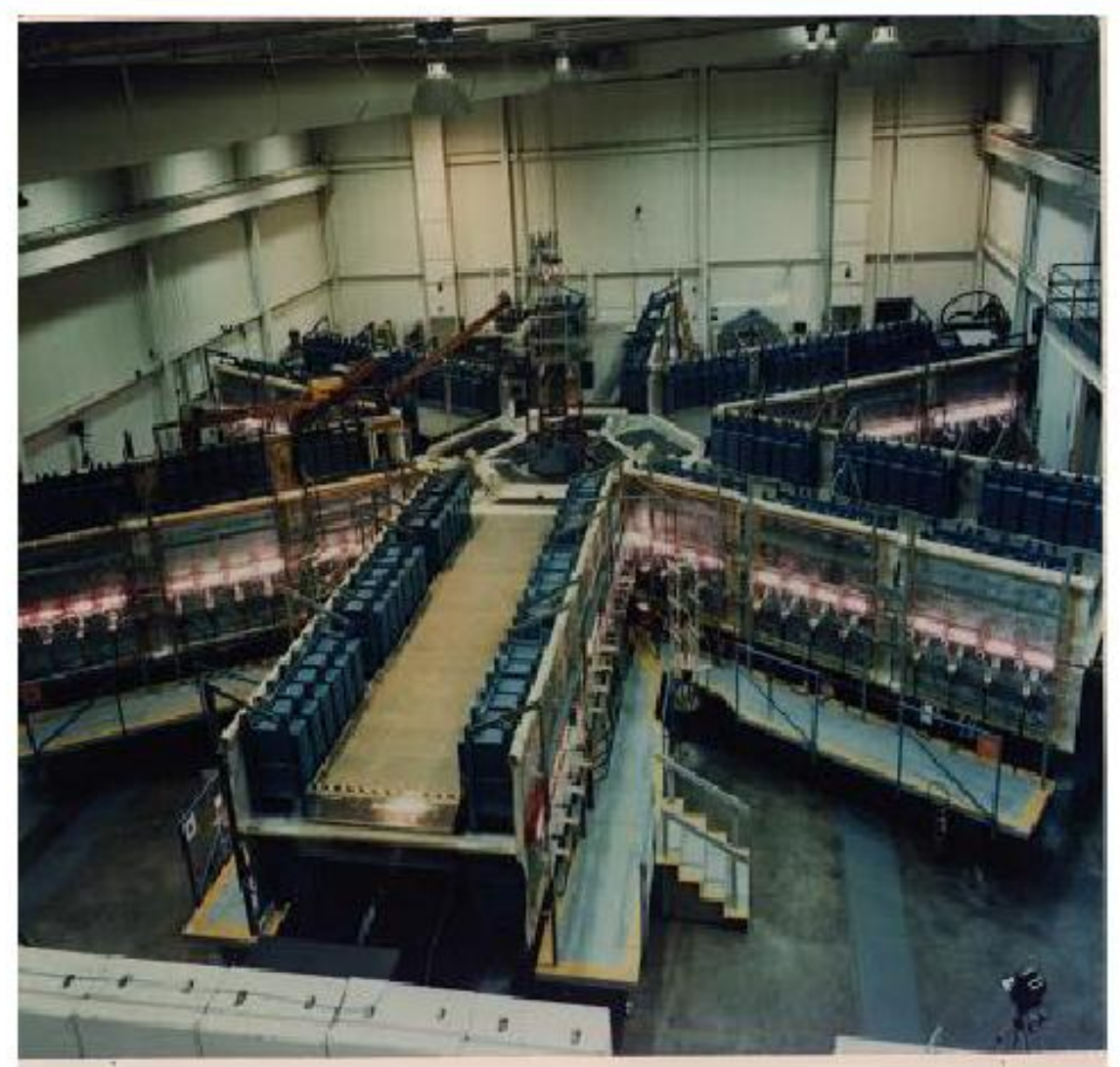

Parameters for magnetic pressure implosions of cylindrical or spherical metal shells (solid liners)

- 80 to $90 \mathrm{kV}, 1300 \mathrm{uF}, 25$ to $45 \mathrm{nH}$

- 11 to 16 Megamp, $\sim 10 \mu \mathrm{sec}$ risetime discharge implodes $10 \mathrm{~cm}$ diameter, $1 \mathrm{~mm}$ thick, 4 to $30 \mathrm{~cm}$ long Al liner in 15 to 24 $\mu \mathrm{sec}$

• e.g., 4.4 MJ energy storage gives $1.5 \mathrm{MJ}$ in liner KE

Shiva Star Capacitor Bank (up to 9 Megajoules, $3 \mu \mathrm{sec}$ ) used for implosion - compression experiments 


\section{The MagLIF concept is a 3 step process*}
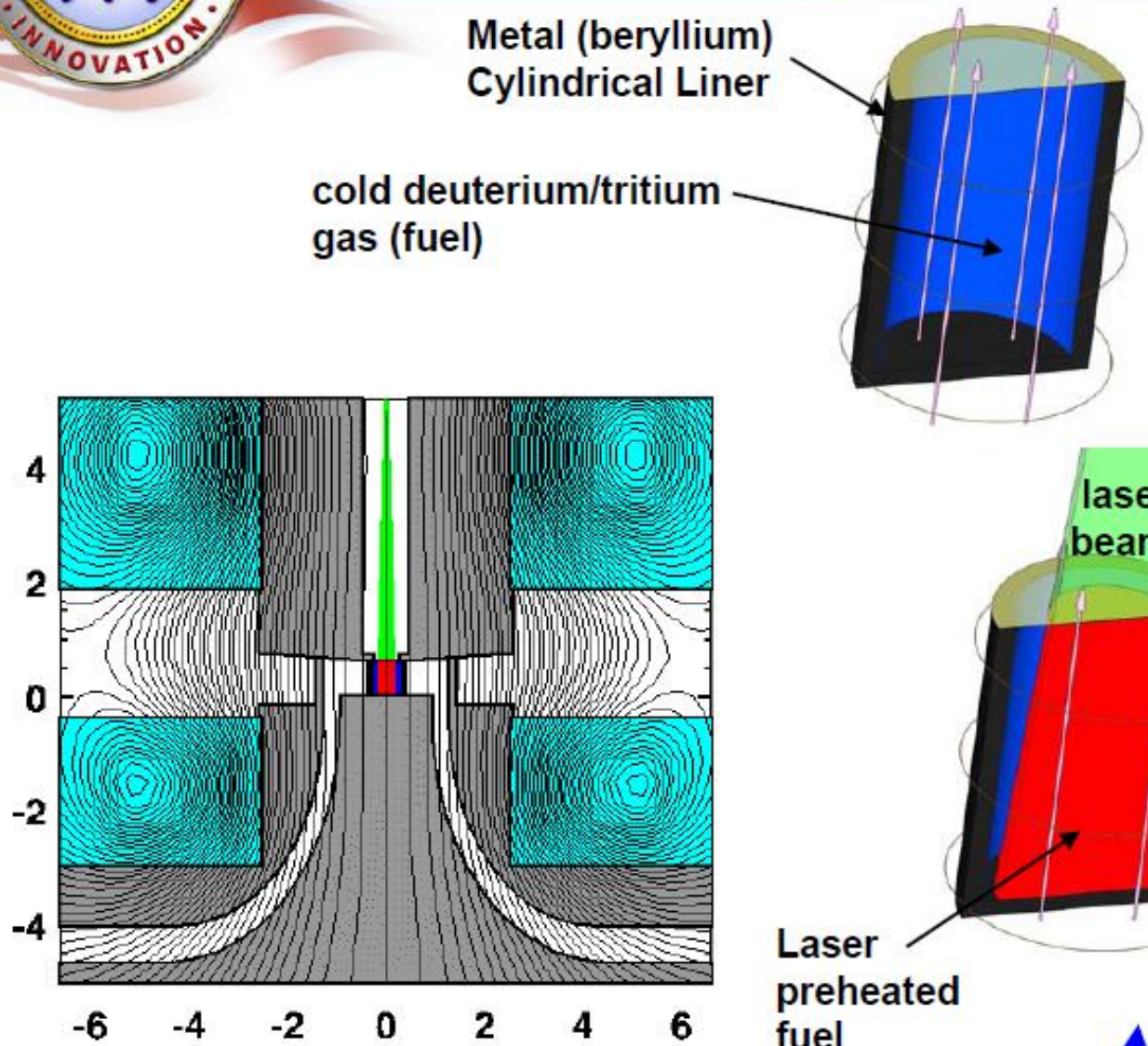

* S.A. Slutz et al. Physics of Plasmas
17, 056303 (2010)

1. An axial magnetic field is applied to inhibit thermal conduction and enhance alpha particle deposition

2. Z Beamlet preheats the fuel

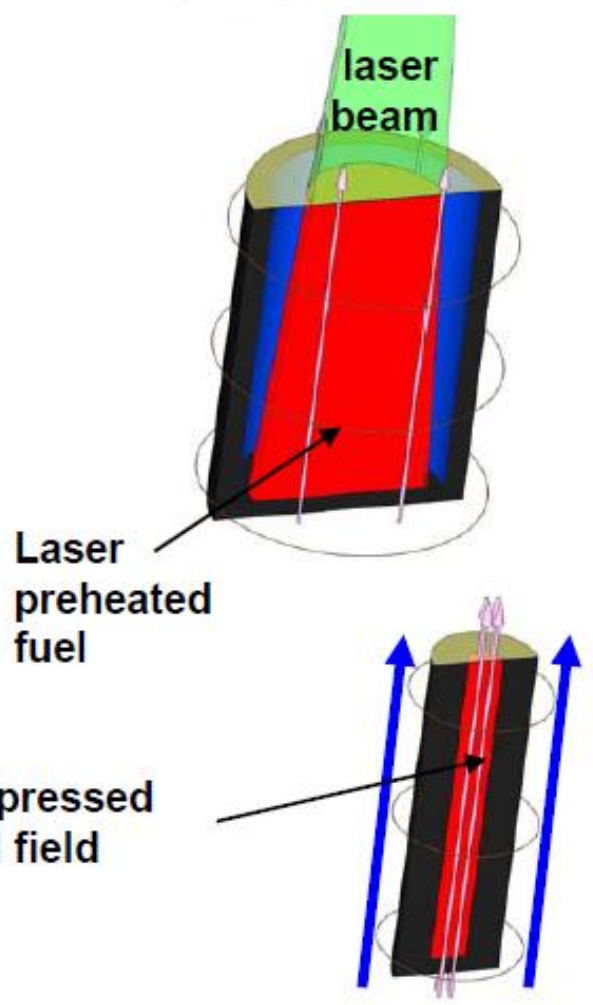

3. The $Z$ accelerator efficiently drives a z-pinch implosion

From S.A. Slutz, 2012 MagLIF Workshop 
For adiabatic compression, preheat reduces the require convergence ratio

For cylindrical system

$$
\begin{aligned}
& V(t)=V_{0}\left[r(t) / r_{0}\right]^{2} \\
& V_{f}=V_{0}\left[r_{f} / r_{0}\right]^{2}=V_{0} C_{R}^{-2}
\end{aligned}
$$

Now, for adiabatic compression: $P V^{r^{r}}=$ constant

$$
\begin{array}{ll}
P_{0} V_{0}=N k T_{0} & P_{f} V_{f}=N k T_{f} \\
\text { so, } &
\end{array}
$$

$$
\frac{T_{f}}{T_{0}}=\frac{P_{f} V_{f}}{P_{i} V_{0}}=\frac{\widehat{P_{f} V_{f}^{5 / 3} \cdot V_{f}^{-2 / 3}}}{P_{0} V_{0}^{5 / 3} \cdot V_{0}^{-2 / 3}}=\frac{V_{0}^{2 / 3}}{V_{f}^{2 / 3}}
$$

and,

$$
\left(\frac{V_{0}}{V_{f}}\right)^{2 / 3}=\left[\left(\frac{r_{0}}{r_{f}}\right)^{2}\right]^{2 / 3}=\left[\frac{r_{0}}{r_{f}}\right]^{4 / 3}
$$

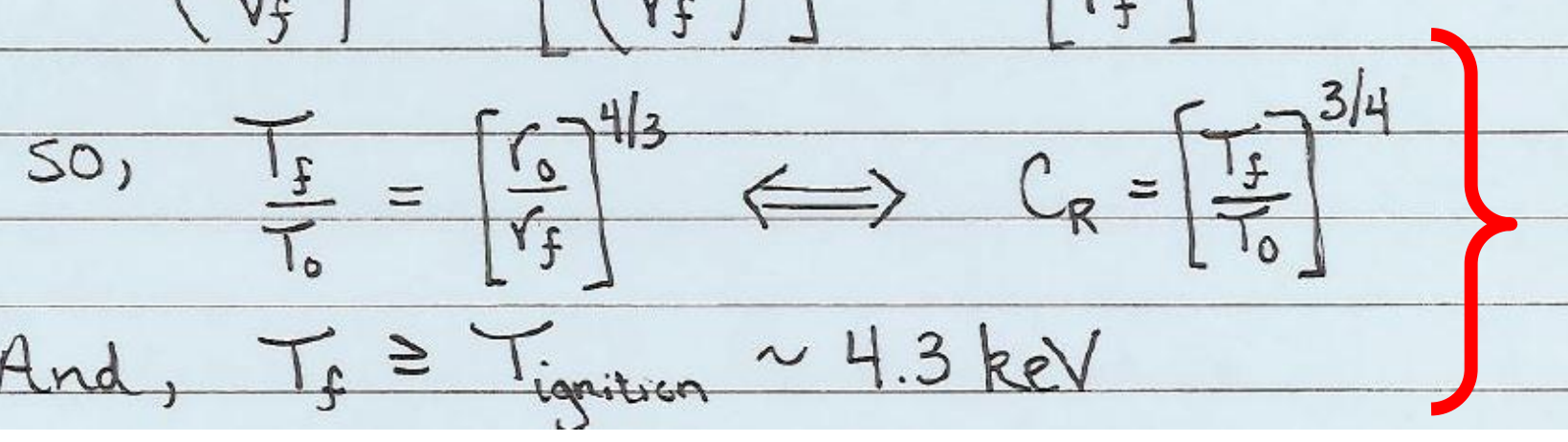

$\mathrm{T}_{0}$ must be 100 's

of $\mathrm{eV}$ or $\mathrm{C}_{\mathrm{R}}$ will

be unobtainable

Candia National Laboratories 


\section{The Z-Beamlet laser could preheat the fuel for experiments on Z}

Conditions for heating of DT gas

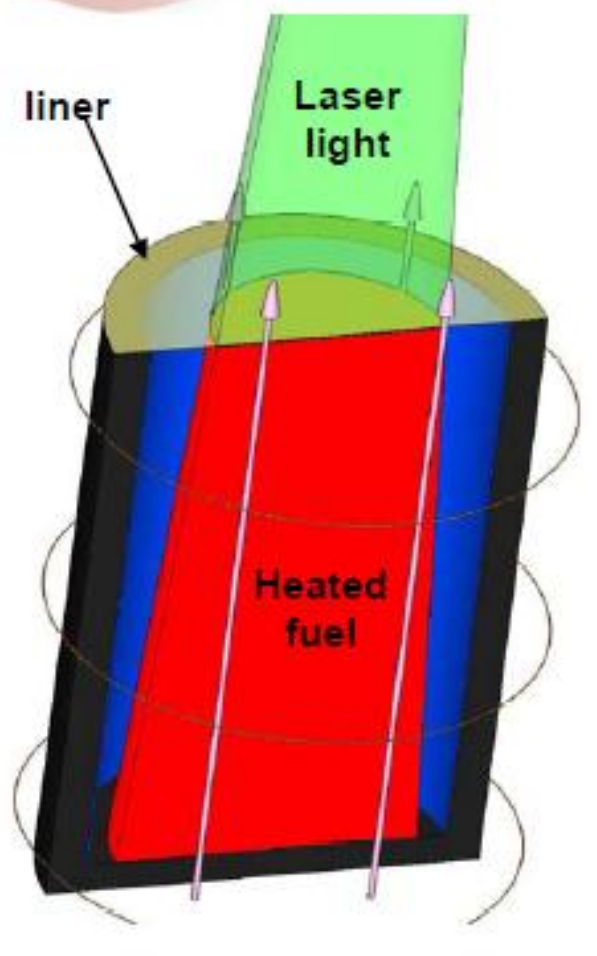

The critical density for green light is $17 \mathrm{mg} / \mathrm{cc}$ in DT

....initial gas density is $2-3 \mathrm{mg} / \mathrm{cc}$ implies absorption by inverse bremsstrahlung

The gas can be held in place by a thin plastic foil ...a $1 \mu$ window will have less areal density than the gas

The total laser energy is modest

$$
\begin{aligned}
& E_{\text {laser }}=\pi r^{2} L \rho C_{V} \theta \quad C_{V} \approx 1.2 \times 10^{8} \mathrm{~J} / \mathrm{g} / \mathrm{keV} \\
& E_{\text {laser }} \approx 3.4\left(\frac{r}{0.3 \mathrm{~cm}}\right)^{2}\left(\frac{L}{1 \mathrm{~cm}}\right)\left(\frac{\rho_{0}}{1 \mathrm{mg} / \mathrm{cc}}\right)\left(\frac{\theta}{0.1 \mathrm{keV}}\right) \mathrm{kJ}
\end{aligned}
$$

From S.A. Slutz, 2012 MagLIF Workshop 


\section{The MRT instability determines performance of MagLIF and other implosion systems}

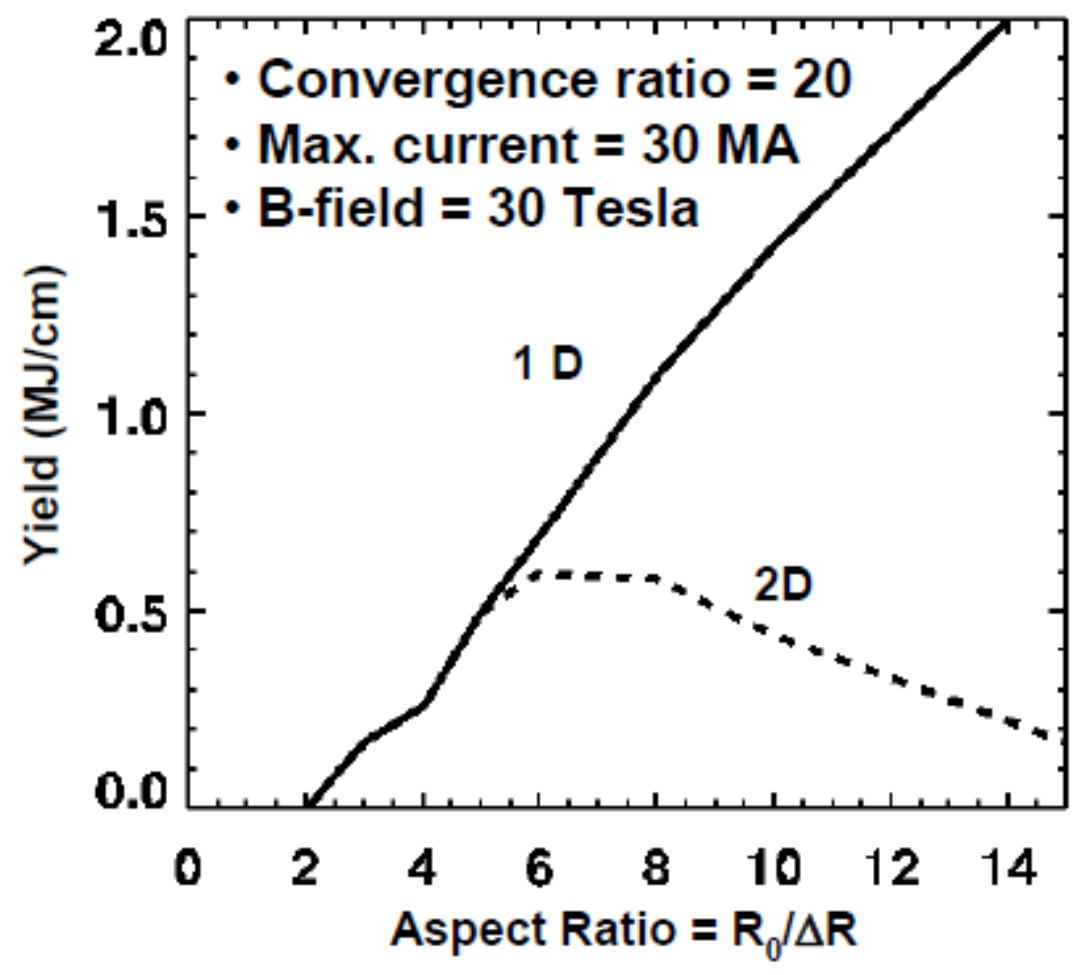

The Magneto-Rayleigh-Taylor instability degrades the yield as the aspect ratio is increased due to decreased liner $\rho r$

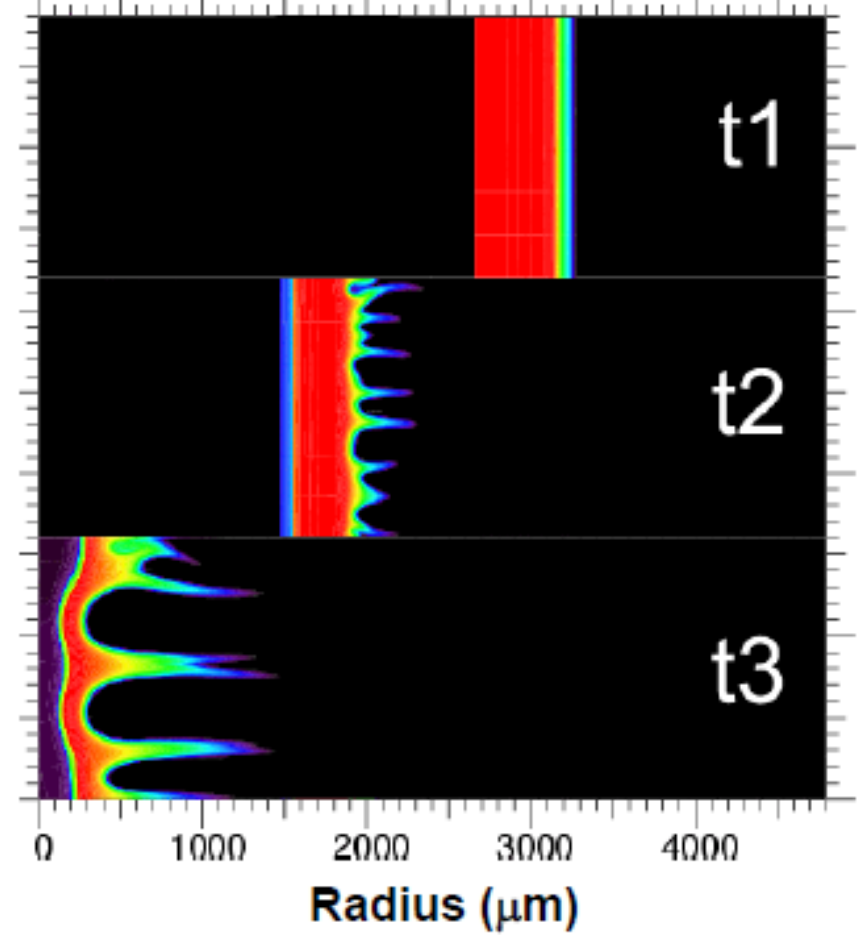

2D Lasnex Be liner simulations $A R=6$ $.60 \mathrm{~nm}$ surface roughness -resolved wavelengths $200-1600 \mu \mathrm{m}$ -wavelengths of 300-600 $\mu \mathrm{m}$ near stagnation 
We have designed, fabricated, and tested a prototype 10-T MagLIF magnetic coil system

10 T MagLIF design with access for 2 frame backlighter

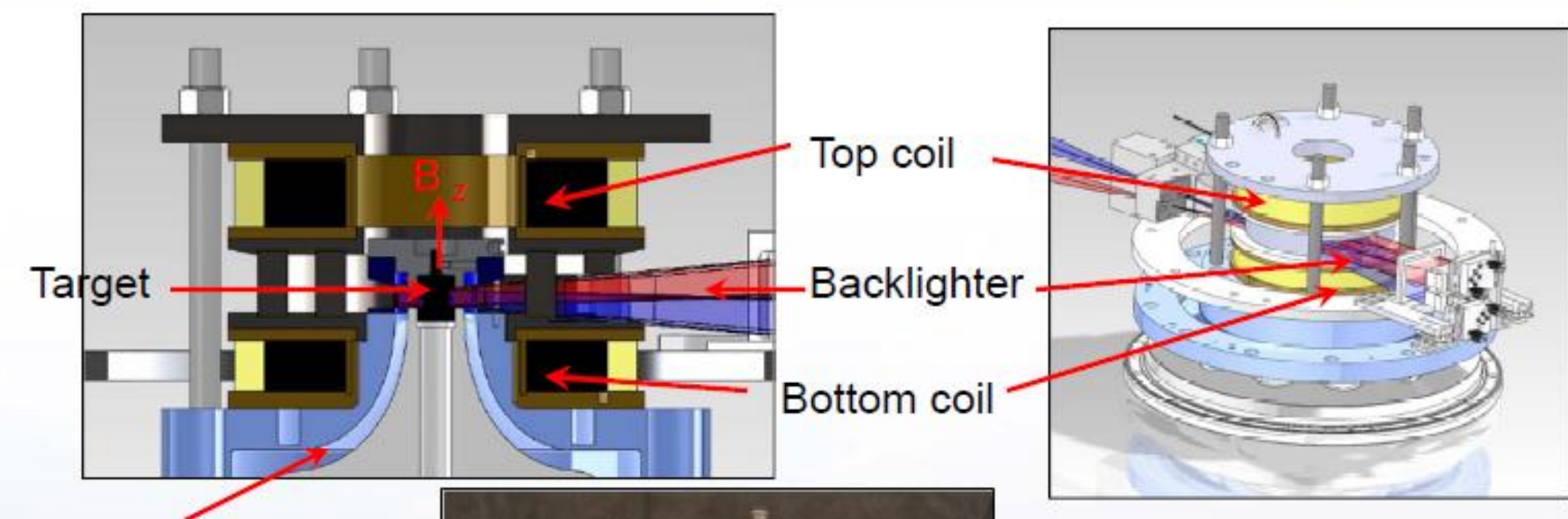

Power feed

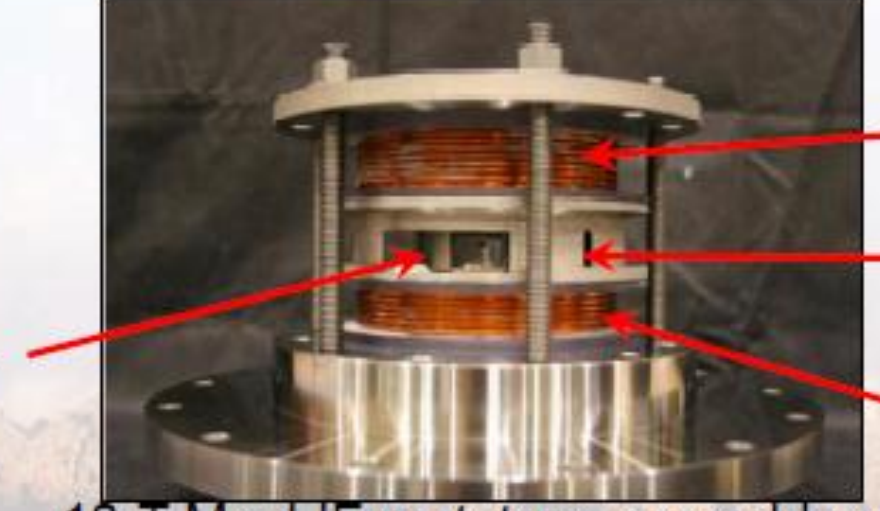

80 turn prototype coil

Backlighter access
10-T MagLIF prototype assembly with

$$
\text { test windings of coils }
$$

VISAR access

60 turn prototype coil SAND2012-0884C 


\section{There is an optimal magnetic field strength for radial propagation}
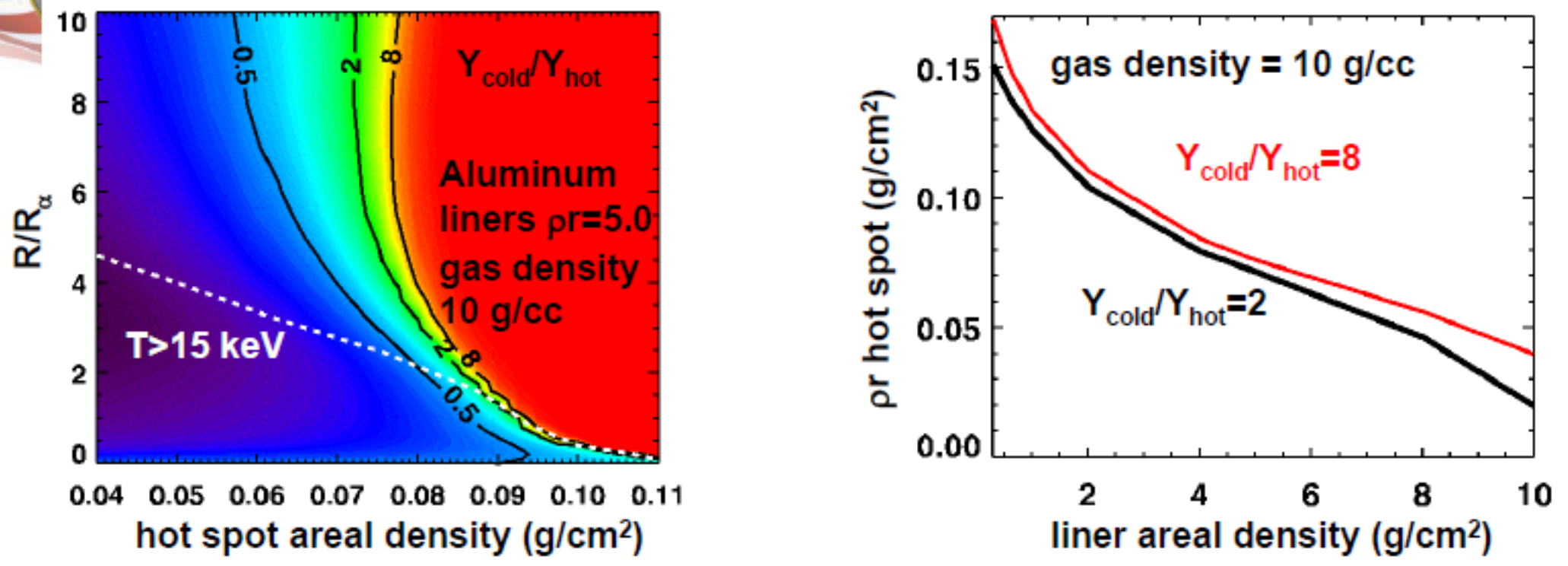

-The inhibition of thermal conduction and alpha transport lowers the hot spot areal density required for for ignition

-Too large a B-field inhibits propagation into the cold fuel

-Minimum hot spot areal density is a weak function of gas density, but is significantly affected by the areal density of the liner

-More confinement time allows slower burn propagation! 


\section{References}

I.R. Lindemuth and R. E. Siemon, Am. J. Phys. 77, 4072009.

M.M. Basko et al., Nuclear Fusion, 40, 59, 2000.

M. Hohenberger et al., Phys. Plasmas, 19, 056306 (2012)

S.A. Slutz et al., Phys. Plasmas, 17, 056303, 2010.

S.A. Slutz and R.A. Vesey, PRL, 108, 025003, 2012.

http://www.sandia.gov/pulsedpower/maglifpres/Agenda.html

S.C. Hsu et al., IEEE Trans. Plasma Sci., 40, No. 5, 1287, 2012.

T.P. Intrator et al., J. Fusion Energy, 27, 57, 2008

S. Atzeni and J. Meryer-Ter-Vehn, "The Physics of Inertial Fusion," Oxford Science Publications, 2004. 


\title{
Electrical Discharge in Gases and LANL's Low-Temperature Plasma Research
}

\author{
Yongho Kim, P-24
}

Aug. 15, 2012 


\section{Outline}

- Early work by Townsend and Paschen

- Electron avalanche ( $\alpha$-mode)

- Breakdown condition ( $\gamma$-mode)

- Breakdown voltage

- Low-temperature plasma properties

- Sheath potential, Vs

- Plasma temperature

- Dynamics of plasma parameters

- LANL's atmospheric-pressure, non-thermal plasma projects

- Atmospheric pressure plasma jet

- Dielectric barrier discharge 


\section{DC low-pressure discharge: the early work by Townsend and Paschen}

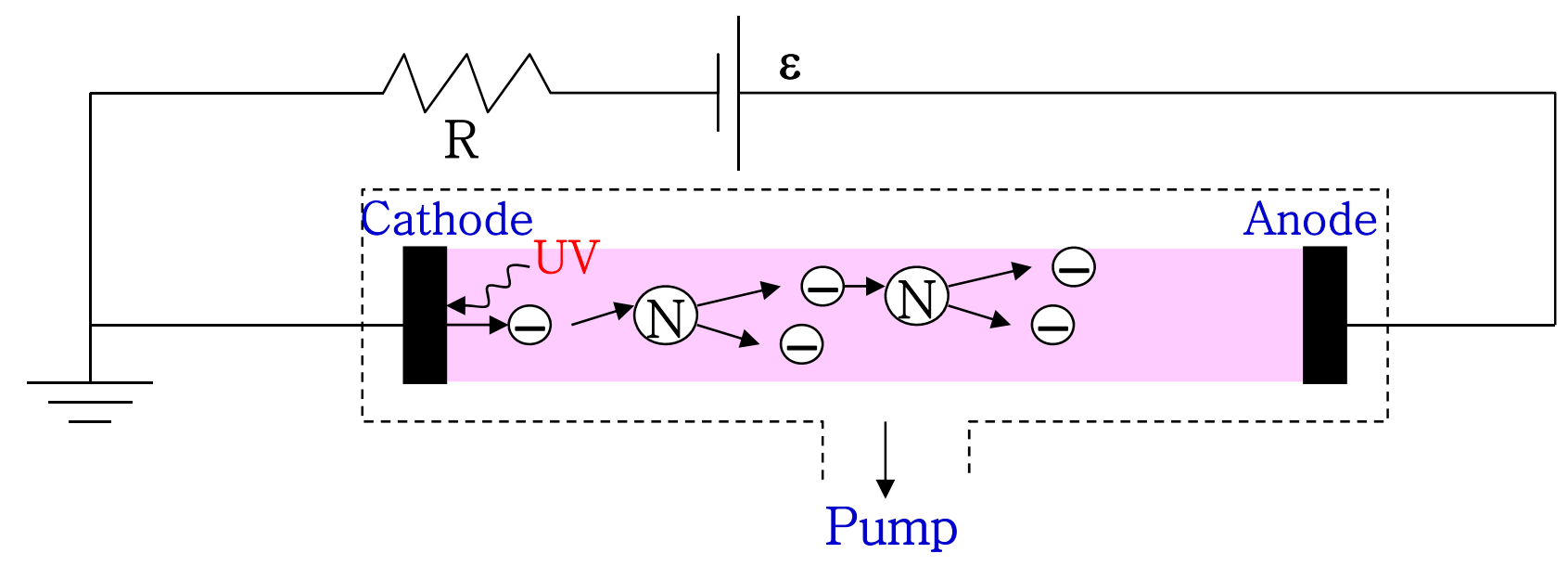

- Electric Circuit and Load Line

$$
\begin{aligned}
& \varepsilon=I R+V \\
& \rightarrow V=-R I+\varepsilon
\end{aligned}
$$

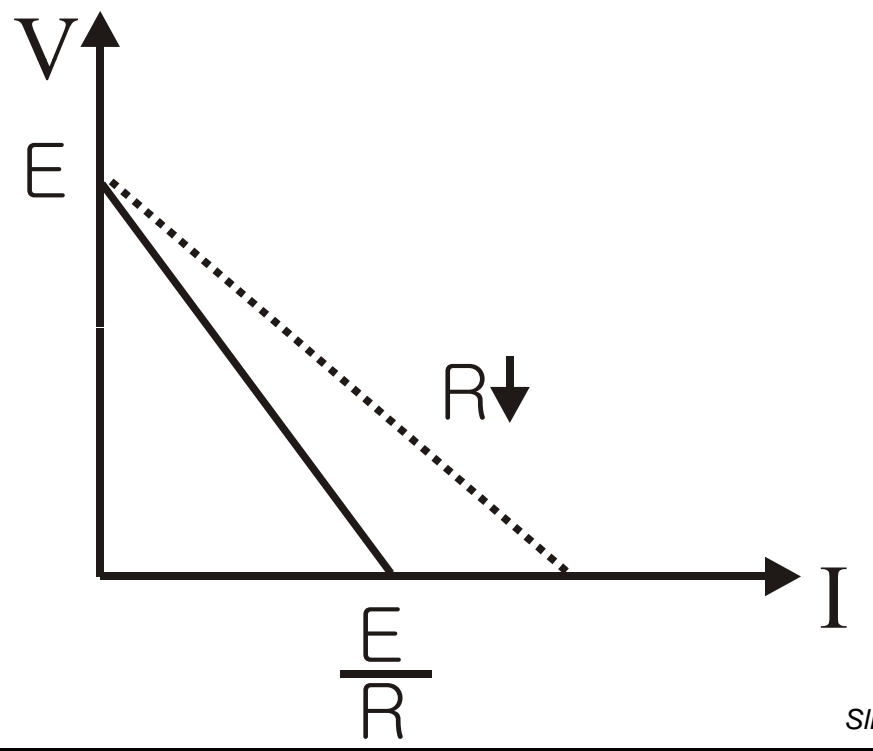




\section{I-V characteristics of DC discharge}

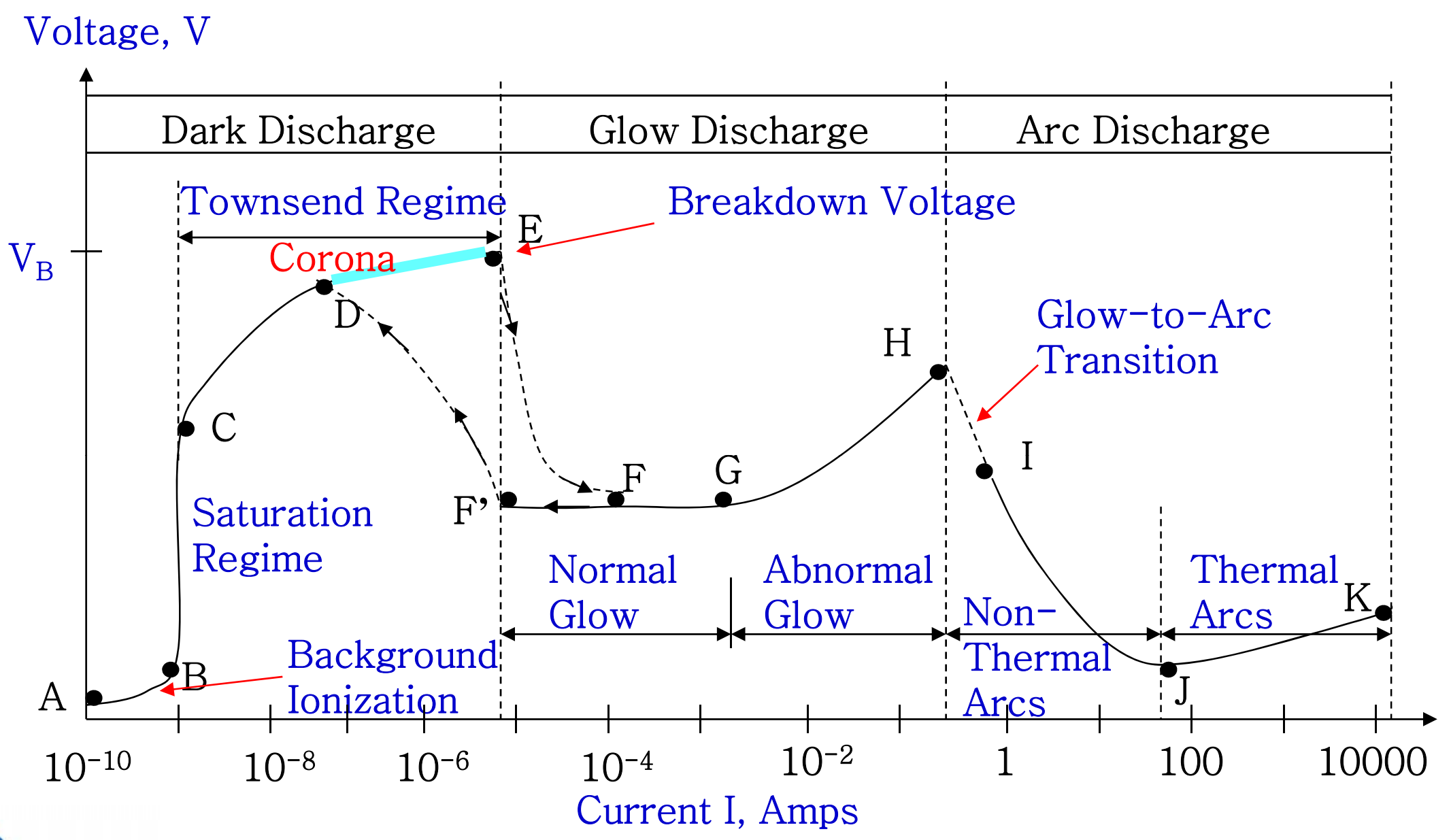




\section{Drift and diffusion of electron in electric field}

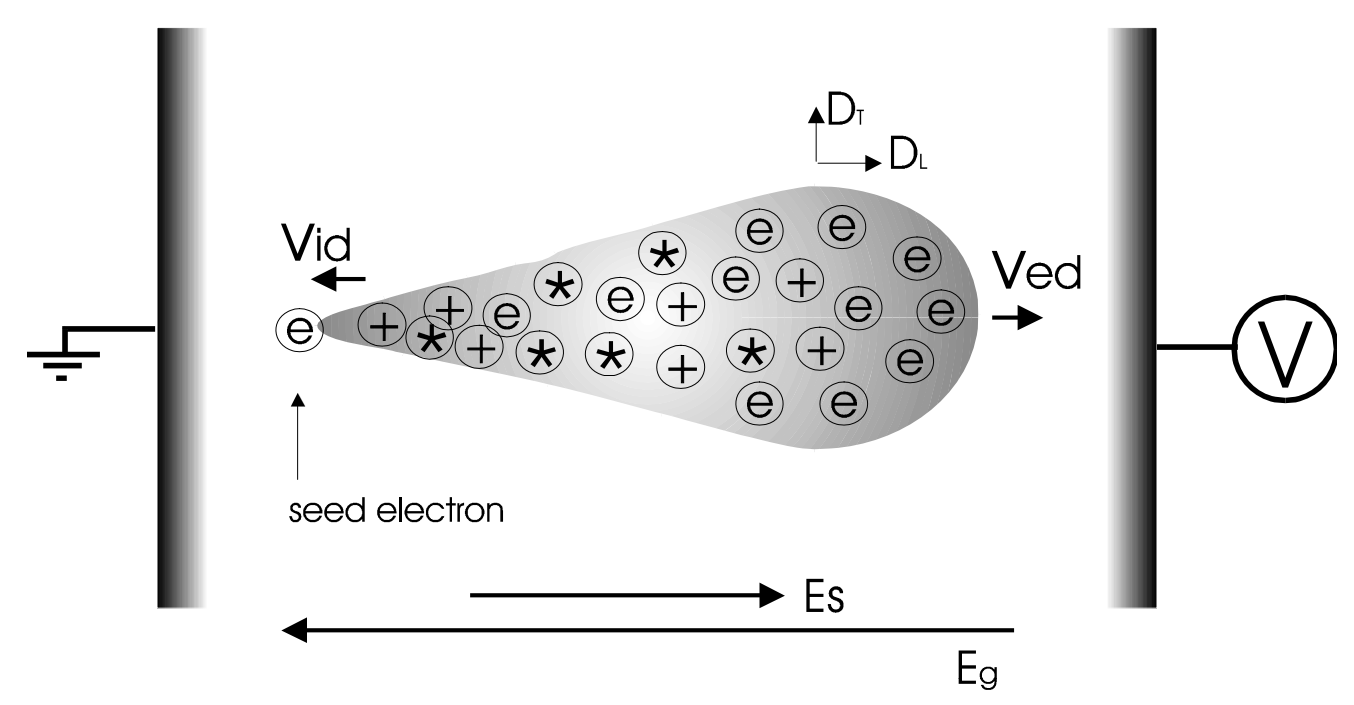

$$
\begin{aligned}
& v_{d}=\mu E=\left(\frac{q}{m v}\right) E=\left(\frac{q}{m}\right)\left(\frac{<v>}{\sigma N}\right) E \quad \propto f(E / p) \\
& D=\frac{k T}{m v}=\left(\frac{k T}{m}\right)\left(\frac{<v>}{\sigma N}\right) \propto f(T / p) \quad \propto \quad f(E / p)
\end{aligned}
$$




\section{Electron collisions in electric field}

\begin{tabular}{|c|c|c|c|}
\hline & electronic reaction & atomic reaction & photon reaction \\
\hline excitation & $\mathrm{e}+\mathrm{A}_{2} \rightarrow \mathrm{A}_{2}{ }^{*}+\mathrm{e}$ & & $\mathrm{hv}+\mathrm{A}_{2} \rightarrow \mathrm{A}_{2}{ }^{*}$ \\
\hline de-excitation & $\mathrm{e}+\mathrm{A}_{2}{ }^{*} \rightarrow \mathrm{A}_{2}+\mathrm{e}$ & & $\mathrm{A}_{2}^{*} \rightarrow \mathrm{A}_{2}+\mathrm{hv}$ \\
\hline dissociation & $\mathrm{e}+\mathrm{A}_{2} \rightarrow 2 \mathrm{~A}+\mathrm{e}$ & $\begin{array}{l}\mathrm{M}^{*}+\mathrm{A}_{2} \rightarrow 2 \mathrm{~A}+\mathrm{M} \\
(\text { Penning dissociation) }\end{array}$ & \\
\hline ionization & $\mathrm{e}+\mathrm{A}_{2} \rightarrow \mathrm{A}_{2}^{+}+2 \mathrm{e}$ & $\begin{array}{c}\mathrm{M}^{*}+\mathrm{A}_{2} \rightarrow \mathrm{A}_{2}^{+}+\mathrm{M}+\mathrm{e} \\
\text { (Penning ionization) }\end{array}$ & $\begin{array}{c}\mathrm{hv}+\mathrm{A}_{2} \rightarrow \mathrm{A}_{2}^{+}+\mathrm{e} \\
\text { (photo ionization) }\end{array}$ \\
\hline attachment & $\mathrm{e}+\mathrm{A}_{2} \rightarrow \mathrm{A}_{2}^{-}$ & & \\
\hline recombination & $\begin{array}{c}\mathrm{e}+\mathrm{A}_{2}^{+} \rightarrow \mathrm{A}_{2} \\
2 \mathrm{e}+\mathrm{A}_{2}^{+} \rightarrow \mathrm{A}_{2}+\mathrm{e}\end{array}$ & $\mathrm{A}^{-}+\mathrm{B}^{+} \rightarrow \mathrm{AB}$ & $\begin{array}{c}\mathrm{A}_{2}^{+}+\mathrm{e} \rightarrow \quad \mathrm{A}_{2}+\mathrm{hv} \\
\text { (radiative recombination) }\end{array}$ \\
\hline
\end{tabular}




\section{Electron avalanche and Townsend coefficient ( $\alpha$-mode)}

- Electron avalanche = rapid electron density growth by electron collisions

- Eqn. of continuity: $\frac{\partial n}{\partial t}+\vec{u} \cdot \nabla n=n n_{a}<\sigma_{i} v>$

- 1-D, steady state case: $\frac{d n}{d x}=\left(\frac{n_{a}<\sigma_{i} v>}{u}\right) n=\alpha n$

$$
\therefore n(x)=n_{o} \exp (\alpha x)
$$

- Townsend coefficient (ionization probability per unit length):

$$
\alpha=n_{a}<\sigma_{i} v>/ u=(A p) \exp (-B p / E)
$$




\section{Townsend breakdown condition}

- Breakdown = onset of self-sustaining discharge produced by secondary emission from cathode by ion bombardments

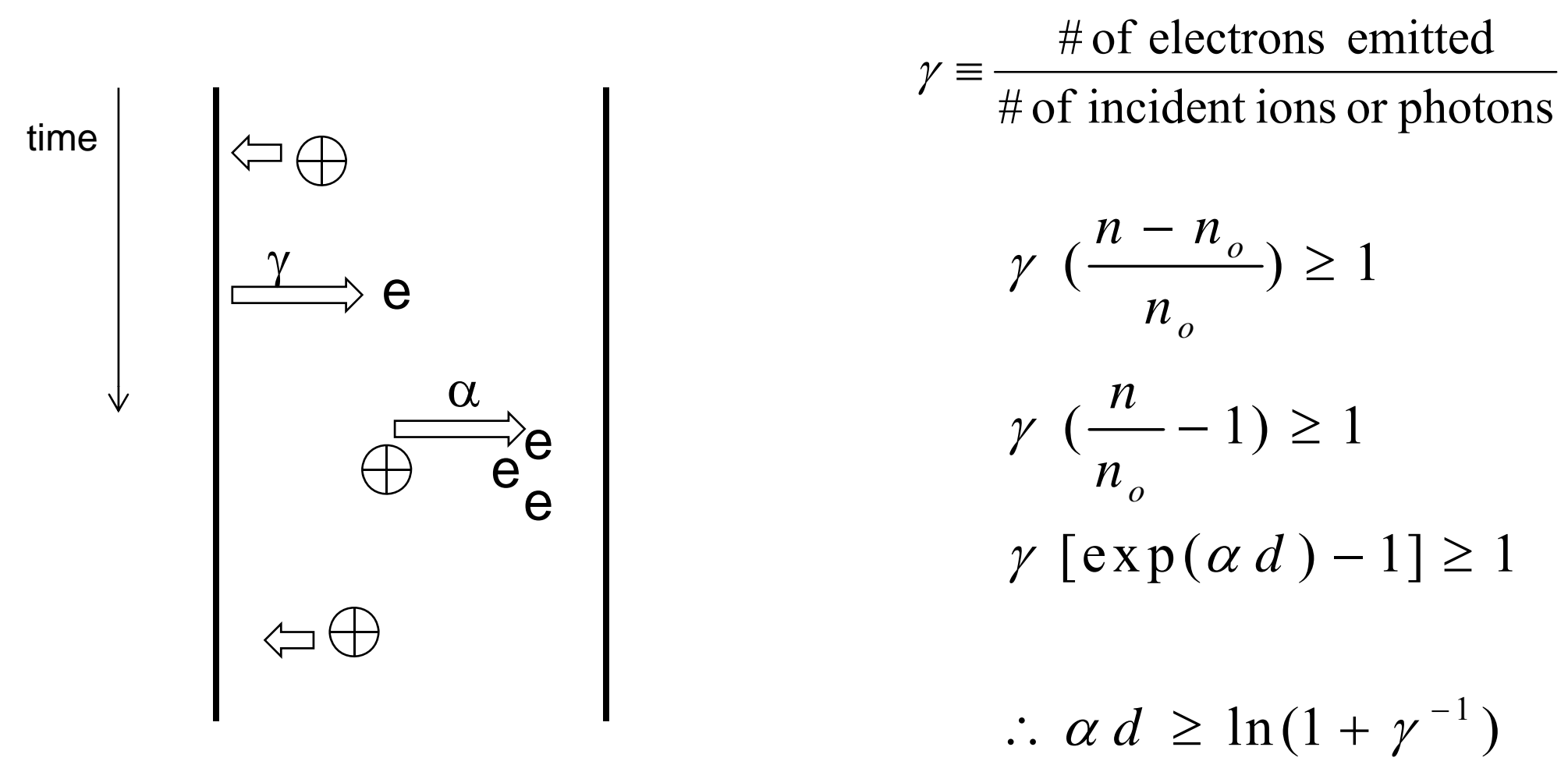




\section{Breakdown voltage and Paschen curve ( $\gamma$-mode)}

$$
\begin{gathered}
\alpha \equiv \frac{N k_{\alpha}}{v_{d}}=A p \exp \left(-\frac{B p}{E}\right) \\
\text { from } \alpha d \geq \ln \left(1+\gamma^{-1}\right)
\end{gathered}
$$

Apd $\exp \left(-\frac{B p}{E}\right) \geq \ln \left(1+\gamma^{-1}\right)$

Apd $\exp \left(-\frac{B p d}{V}\right) \geq \ln \left(1+\gamma^{-1}\right)$

$$
\therefore \quad V \geq \frac{B p d}{\ln \left(\frac{A p d}{\ln \left(1+\gamma^{-1}\right)}\right)}
$$

\begin{tabular}{cccll}
\hline Gas & Cathode $\begin{array}{l}\mathrm{V}_{\text {B,min }} \\
\text { (V) }\end{array}$ & $\begin{array}{c}(\mathrm{pd})_{\min } \\
(\mathrm{mT} \text { m) }\end{array}$ & Reference \\
\hline Air & & 360 & 5.7 & Brown (1966) \\
$\mathrm{H}_{2}$ & $\mathrm{Pt}$ & 295 & 12.5 & Cobine (1958) \\
$\mathrm{He}$ & $\mathrm{Fe}$ & 150 & 25 & Cobine (1958) \\
$\mathrm{N}_{2}$ & $\mathrm{Fe}$ & 275 & 7.5 & Cobine (1958) \\
$\mathrm{O}_{2}$ & $\mathrm{Fe}$ & 450 & 7.0 & Cobine (1958) \\
\hline
\end{tabular}

Relative breakdown voltage, $\mathrm{Y}$

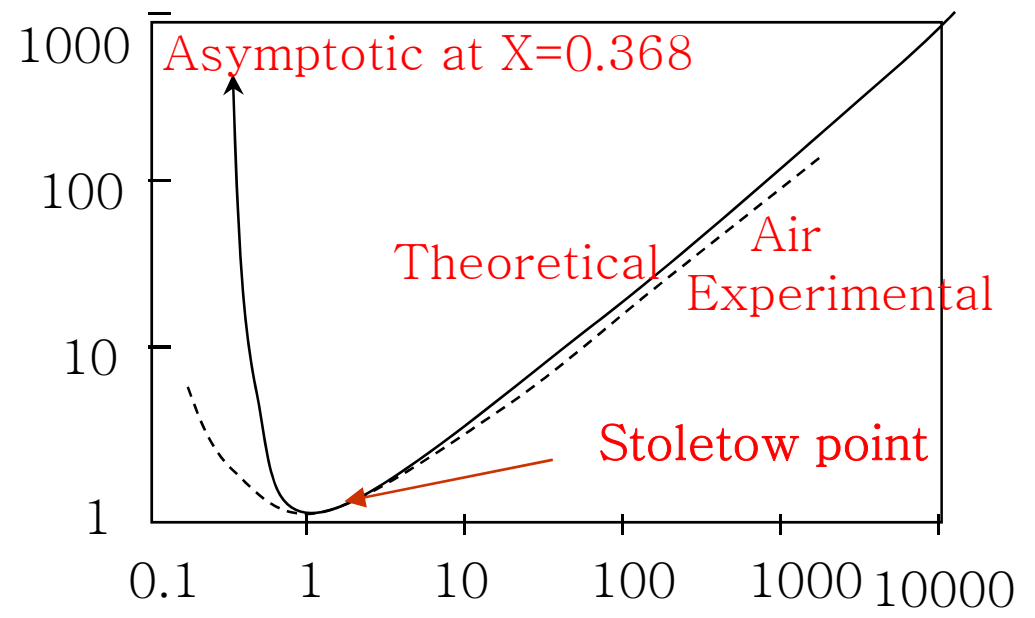

Relative breakdown parameter, $\mathrm{X}$ 


\section{Outline}

- Early work by Townsend and Paschen

- Electron avalanche ( $\alpha$-mode)

- Breakdown condition ( $\gamma$-mode)

- Breakdown voltage

- Low-temperature plasma properties

- Sheath potential, Vs

- Plasma temperature

- Dynamics of plasma parameters

- LANL's atmospheric-pressure, non-thermal plasma projects

- Atmospheric pressure plasma jet

- Dielectric barrier discharge 


\section{How about potential profile inside DC discharge tube}

- - Potential profile before discharge

— Potential profile during discharge

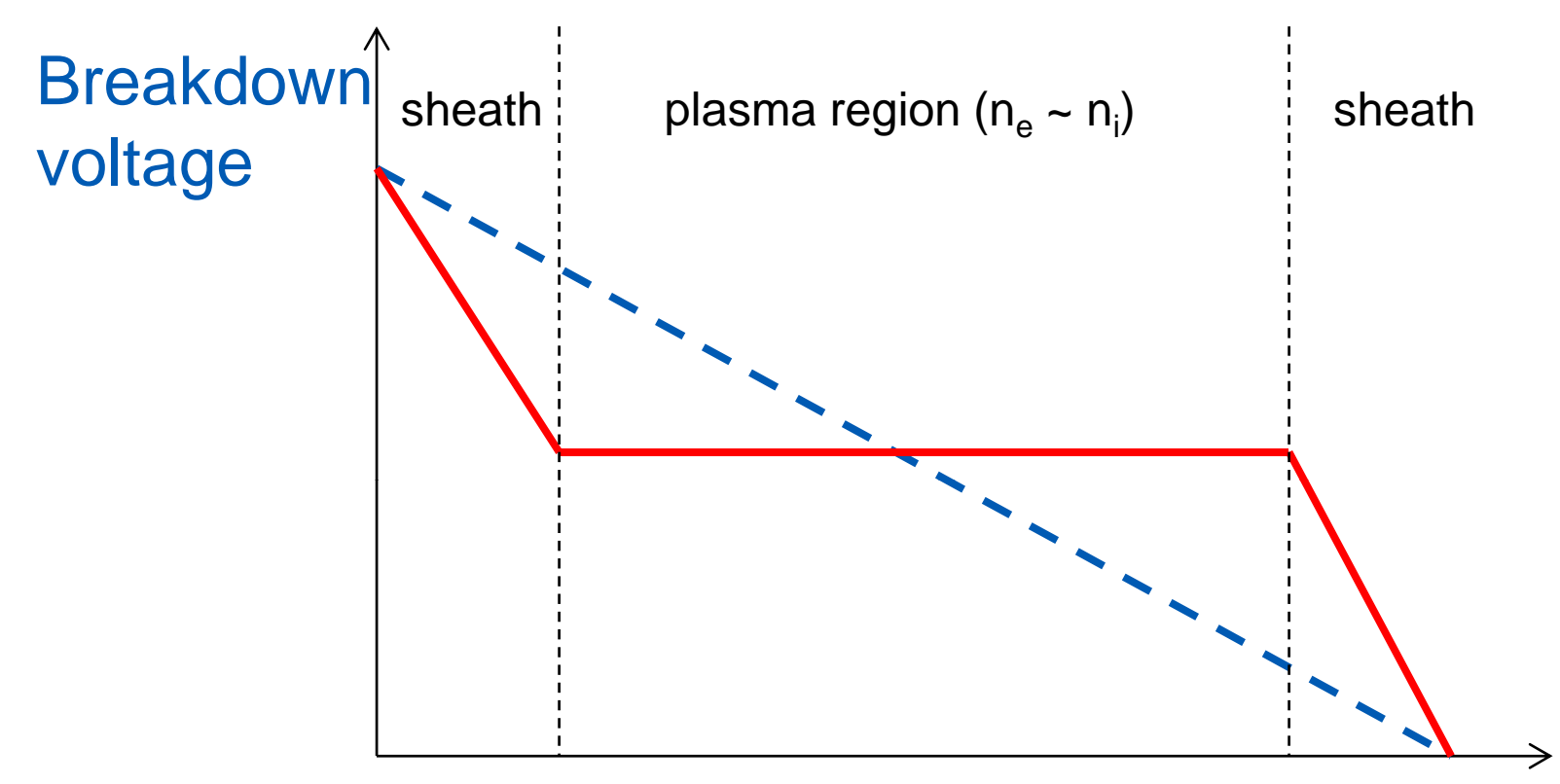

anode

cathode 


\section{How to know plasma sheath potential $\left(\mathrm{V}_{\mathrm{s}}\right)$ ?}

- Ion Flux: The ion flux to a solid object is determined by the Bohm velocity (or sound speed) of the ion,

$$
\Gamma_{i}=u_{B} n_{i}=\sqrt{\frac{k T_{e}}{m_{i}}} n_{i}
$$

- Electron Flux: Only the most energetic electrons can overcome the sheath potential,

$$
\text { - } \Gamma_{e}=1 / \underbrace{\mathrm{n}_{e}<\mathrm{v}_{e}}_{\text {flux to surface }}>\operatorname{exp(q\mathrm {V}_{\mathrm {s}}} / \underbrace{\left.p \mathrm{~T}_{\mathrm{e}}\right)}_{\text {Boltzmann factor }}
$$

- $\mathbf{V}_{\mathbf{s}}$ is determined by ion flux $=$ electron flux, $V_{S}=-T_{e} \ln \left(\frac{m_{i}}{2 m_{e}}\right) \sim-5 T_{e}$ 


\section{After breakdown, plasma temperature is determined}

- kinetic energy exchange $=$ energy gain by field

$$
\begin{aligned}
& \frac{3}{2} k\left(T_{e}-T_{h}\right) \frac{2 m_{e}}{m_{h}}=e E v_{d} t_{e} \\
&=e E\left(\frac{e}{m v} E\right)\left(\frac{\lambda_{e}}{v_{e}}\right) \\
& \Rightarrow \frac{T_{e}-T_{h}}{T_{e}}=\frac{\pi m_{h}}{24 m_{e}} \frac{\left(\lambda_{e} e E\right)^{2}}{\left(k T_{e}\right)^{2}} \propto f(E / p)
\end{aligned}
$$

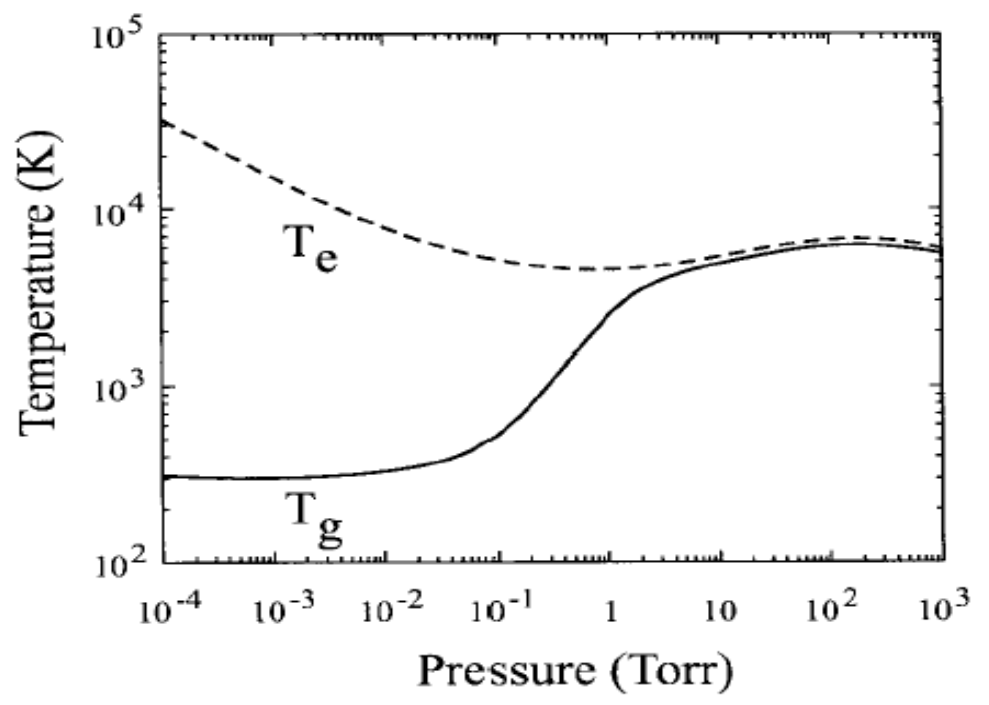




\section{In reality, RF discharge replaces DC discharge to minimize electrode contamination and other issues}

Capacitively-coupled rf discharge (before breakdown)

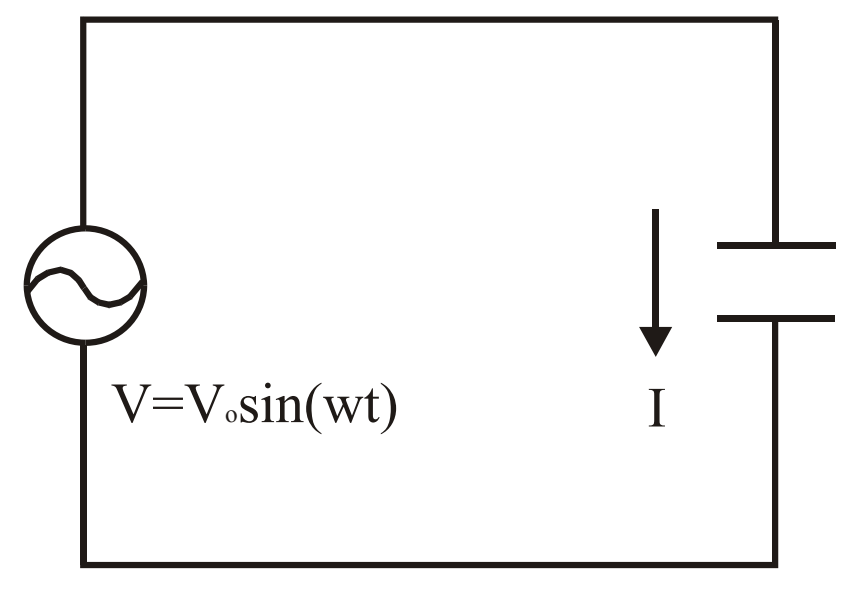

$$
\begin{aligned}
& I=\frac{d Q}{d t}=\frac{d}{d t}\left(C_{g} V\right) \\
& =C_{g} w V_{0} \cos (w t) \\
& =C_{g} w V_{0} \sin \left(w t+\frac{\pi}{2}\right) \\
& =j w C_{g} V
\end{aligned}
$$

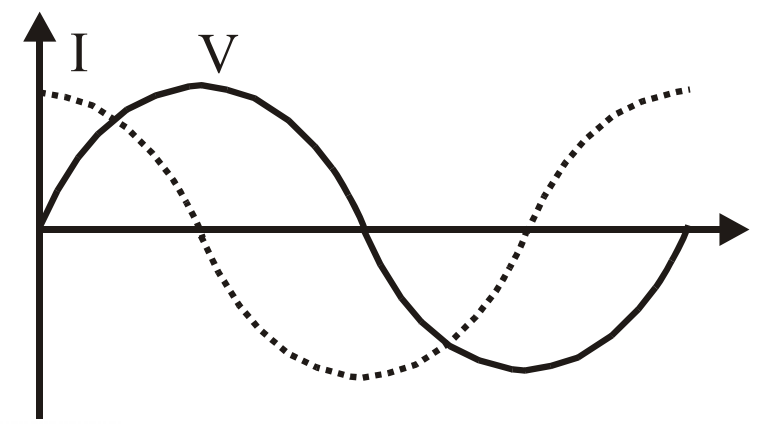

$$
Z_{c}=\frac{V}{I}=\frac{V}{j w C_{g} V}=-j \frac{1}{w C_{g}}
$$




\section{After breakdown, impedance of rf discharge is changed by plasma resistance}

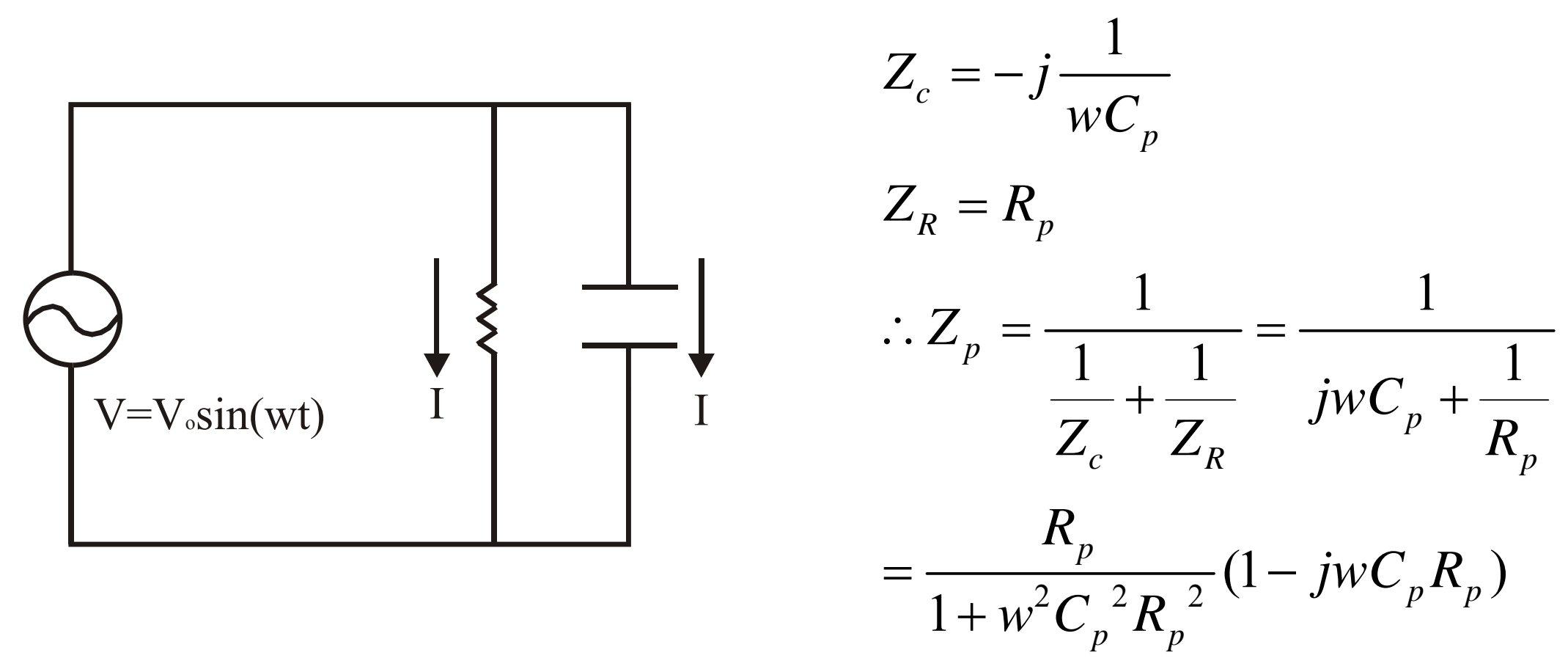




\section{Dynamics of rf discharge operation is that plasma properties are varied as a function of electron density}

Plasma capacitance $\quad C_{p}=\varepsilon_{p} \frac{A_{g}}{d_{g}}=\varepsilon_{o}\left(1-\sigma_{p}\right) \frac{A_{g}}{d_{g}}$
$\sigma_{p}=\frac{w_{p}^{2}}{w^{2}+v_{m}^{2}} \quad, w p=\sqrt{\frac{n e^{2}}{m \varepsilon_{o}}}, v_{m}=N \sigma(v) v$

Plasma resistance

$$
R_{p}=\frac{d_{g}}{\sigma_{p} A_{g}}
$$




\section{Outline}

- Early work by Townsend and Paschen

- Electron avalanche ( $\alpha$-mode)

- Breakdown condition ( $\gamma$-mode)

- Breakdown voltage

- Low-temperature plasma properties

- Sheath potential, Vs

- Plasma temperature

- Dynamics of plasma parameters

- LANL's atmospheric-pressure, non-thermal plasma projects

- Atmospheric pressure plasma jet

- Dielectric barrier discharge 


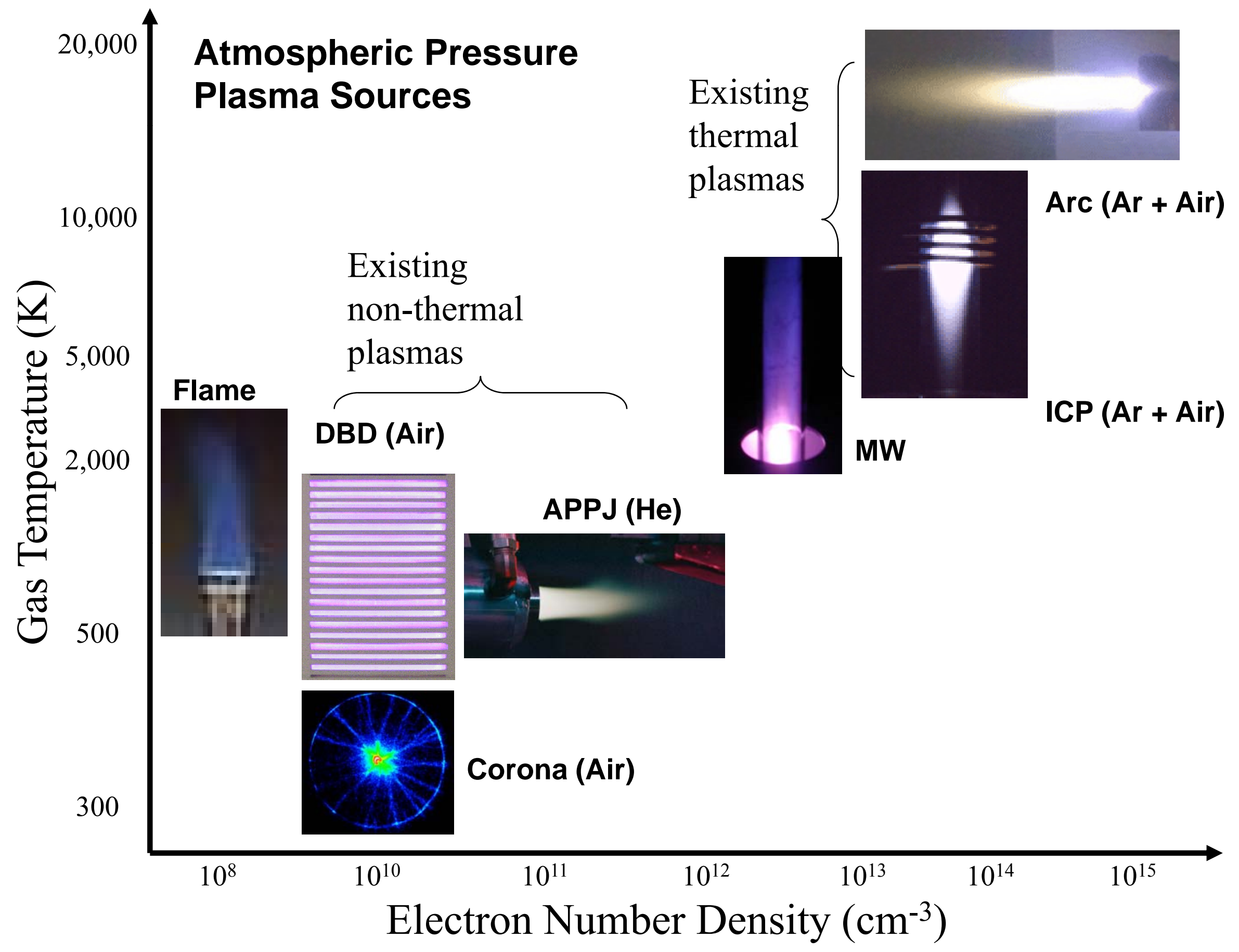




\section{Existing Atmospheric Pressure Plasmas}

\begin{tabular}{|c|c|c|c|}
\hline \multirow{3}{*}{ Electron Pressure } & \multirow{2}{*}{$\begin{array}{l}\text { Thermal } \\
\text { Plasma }\end{array}$} & \multicolumn{2}{|c|}{ Non-Thermal Plasma } \\
\hline & & Transient & Stationary \\
\hline & & & \\
\hline $\begin{array}{l}\text { Electron } \\
\text { Temperature } \\
(\mathrm{eV})\end{array}$ & $\sim 1$ & $\sim 10$ & $\sim 2$ \\
\hline $\begin{array}{l}\text { Gas Temperature } \\
(\mathrm{K})\end{array}$ & $5000 \sim 15000$ & $\sim 300$ & $\sim 300$ \\
\hline $\begin{array}{l}\text { Electron Density } \\
\left(\mathrm{cm}^{-3}\right)\end{array}$ & $10^{16} \sim 10^{19}$ & $10^{10} \sim 10^{12}$ & $10^{11} \sim 10^{12}$ \\
\hline \multirow{3}{*}{ Sources } & DC Arc Torch & \multirow{3}{*}{$\begin{array}{l}\text { PCD } \\
\text { DBD }\end{array}$} & APPJ \\
\hline & RF-ICP & & MHCD \\
\hline & Microwave & & OAUGDP \\
\hline
\end{tabular}

DBD: Dielectric Barrier Discharge, APPJ: Atmospheric Pressure Plasma Jet 


\section{Atmospheric Pressure Plasma Jet (APPJ)}
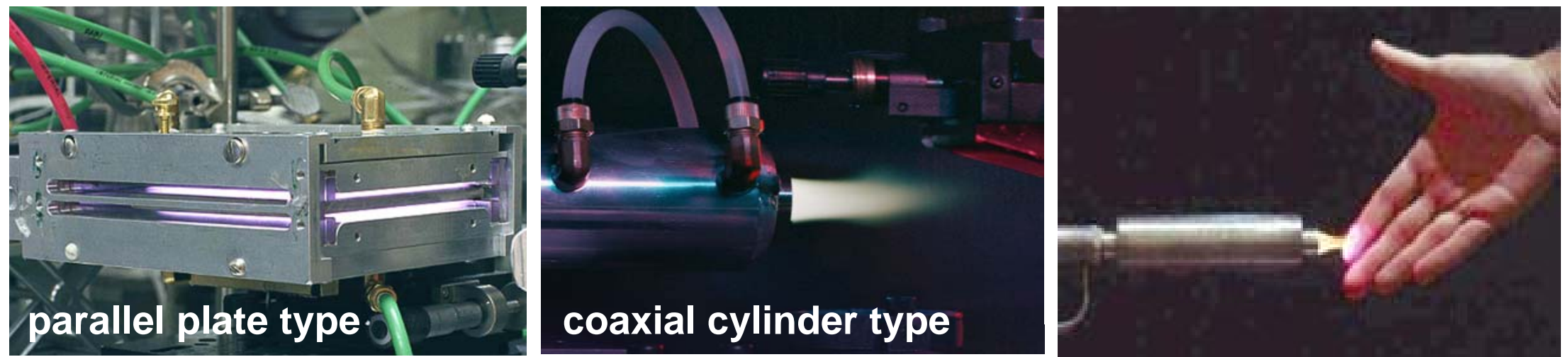

- Capacitively coupled if discharge operated at atmospheric pressure

- free of arcing (streamers) without dielectric barrier

- rf $\alpha$-mode operation at $13.56 \mathrm{MHz}$

- helium based $(\sim 96 \%)+$ additive gas $\left(\sim 4 \% \mathrm{O}_{2}, \mathrm{CF}_{4}\right)$

- narrow gap $(1 \sim 3 \mathrm{~mm})$ operation

- Volumetric non-equilibrium plasma

- oscillating steady state discharge with $\mathrm{N}_{\mathrm{e}} \sim 10^{11} \mathrm{~cm}^{-3}$

- $\mathrm{T}_{\mathrm{e}}(1 \sim 2 \mathrm{eV})>>\mathrm{T}_{\text {gas }}\left(50 \sim 300{ }^{\circ} \mathrm{C}\right)$

- abundant radicals/metastables in plasma and effluent 


\section{APPJ is designed for Decontamination of Actinides (Pu and $U$ )}

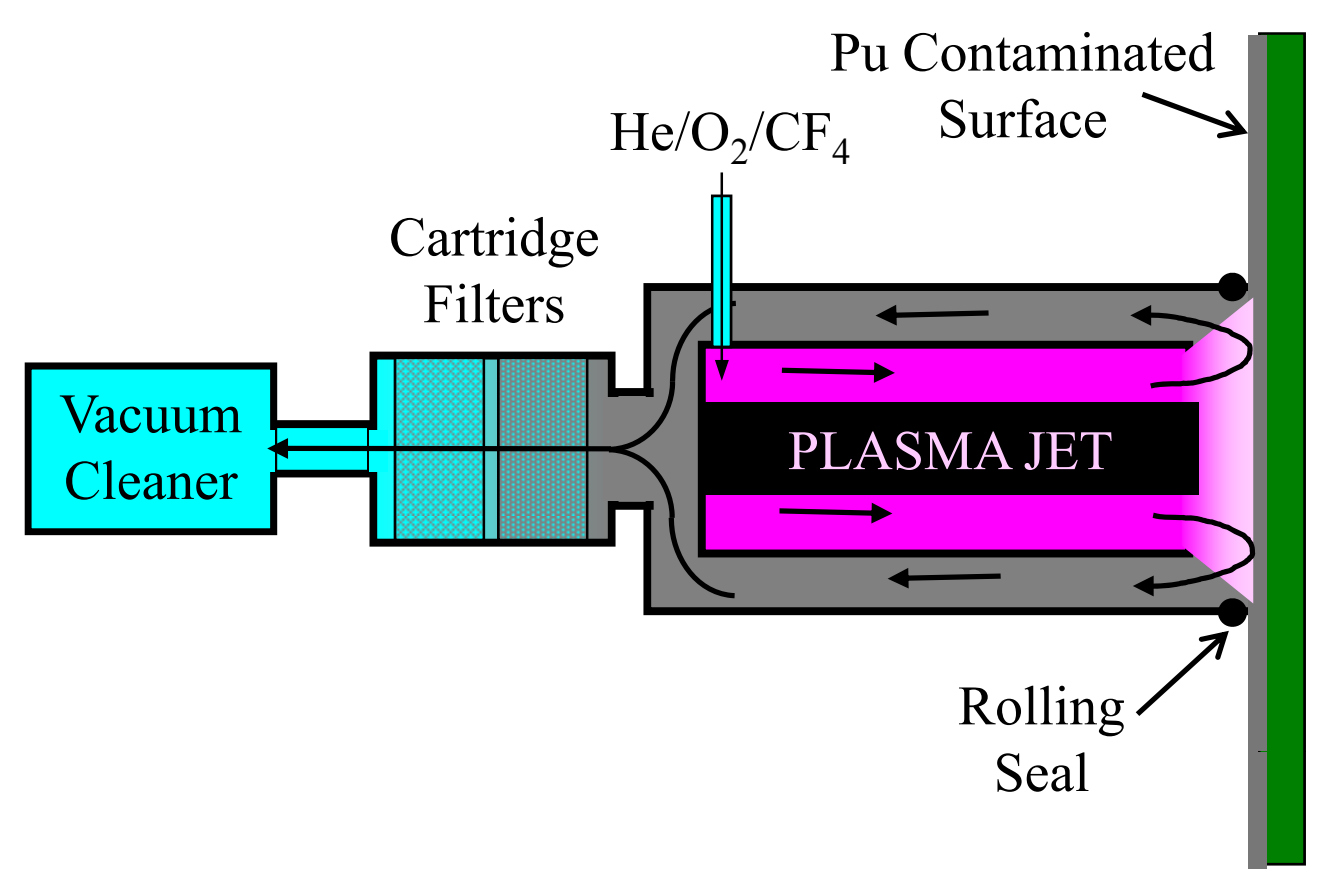

(1) In plasma, energetic electrons dissociate $\mathrm{CF}_{4}$ forming $\mathrm{F}$ atoms

(2) Atomic Fluorine may etch contamination: $\mathrm{Pu}(\mathrm{s})+6 \mathrm{~F}(\mathrm{~g}) \Rightarrow \mathrm{PuF}_{6}(\mathrm{~g})$

(3) Volatile byproducts (e.g., $\mathrm{PuF}_{6}$ ) can be captured in adsorbent filters

(4) We can re-cycle Pu and reduce Contaminated Volume 


\section{Dielectric Barrier Discharge (DBD)}

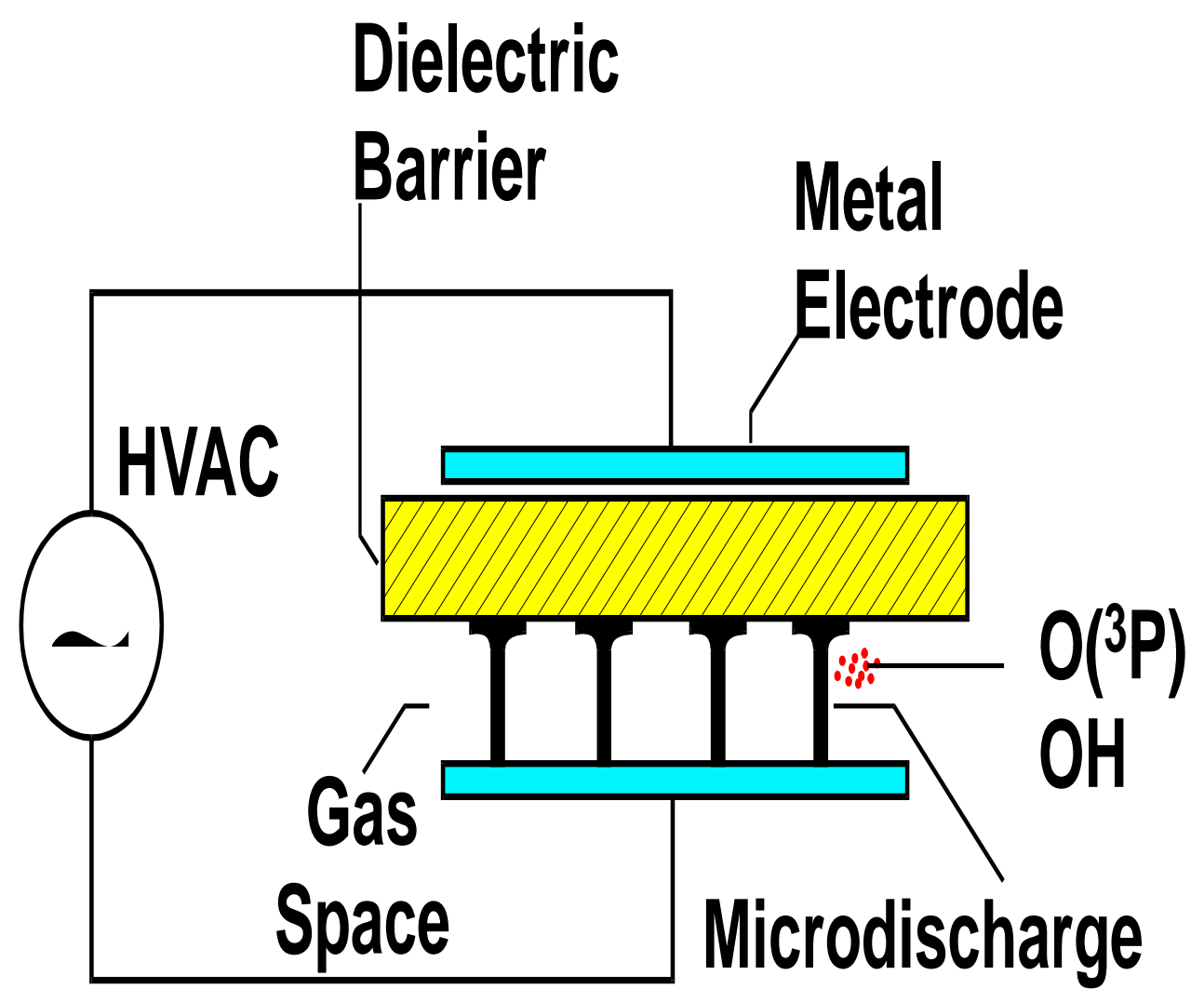

Schematic Drawing by Louis Rosocha
Top view

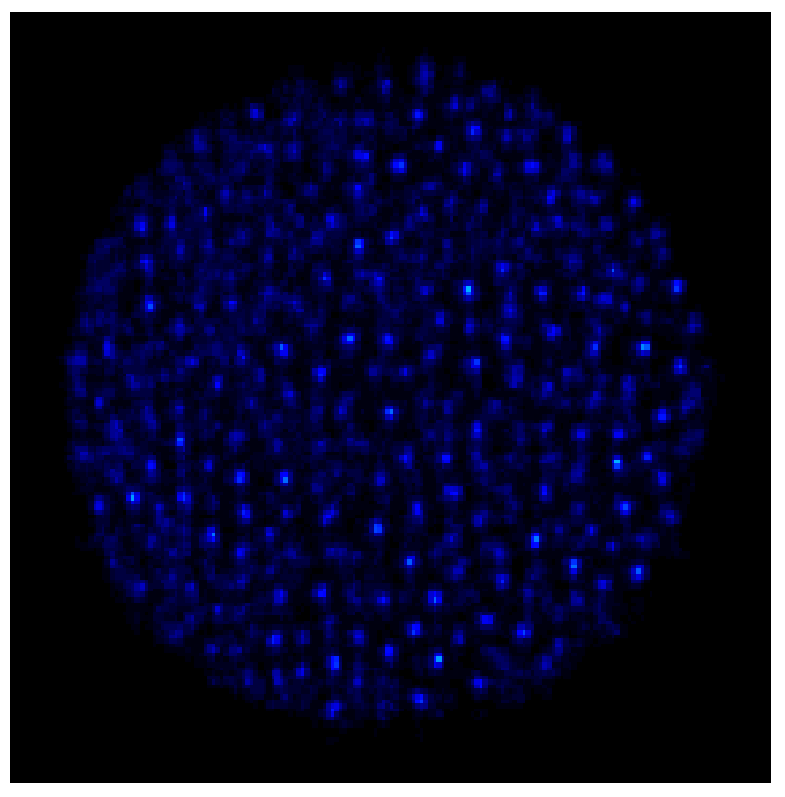

ICCD image of microdischarge channels taken by Yongho Kim in 2002 


\section{Can Plasma improve Combustion Efficiency?}

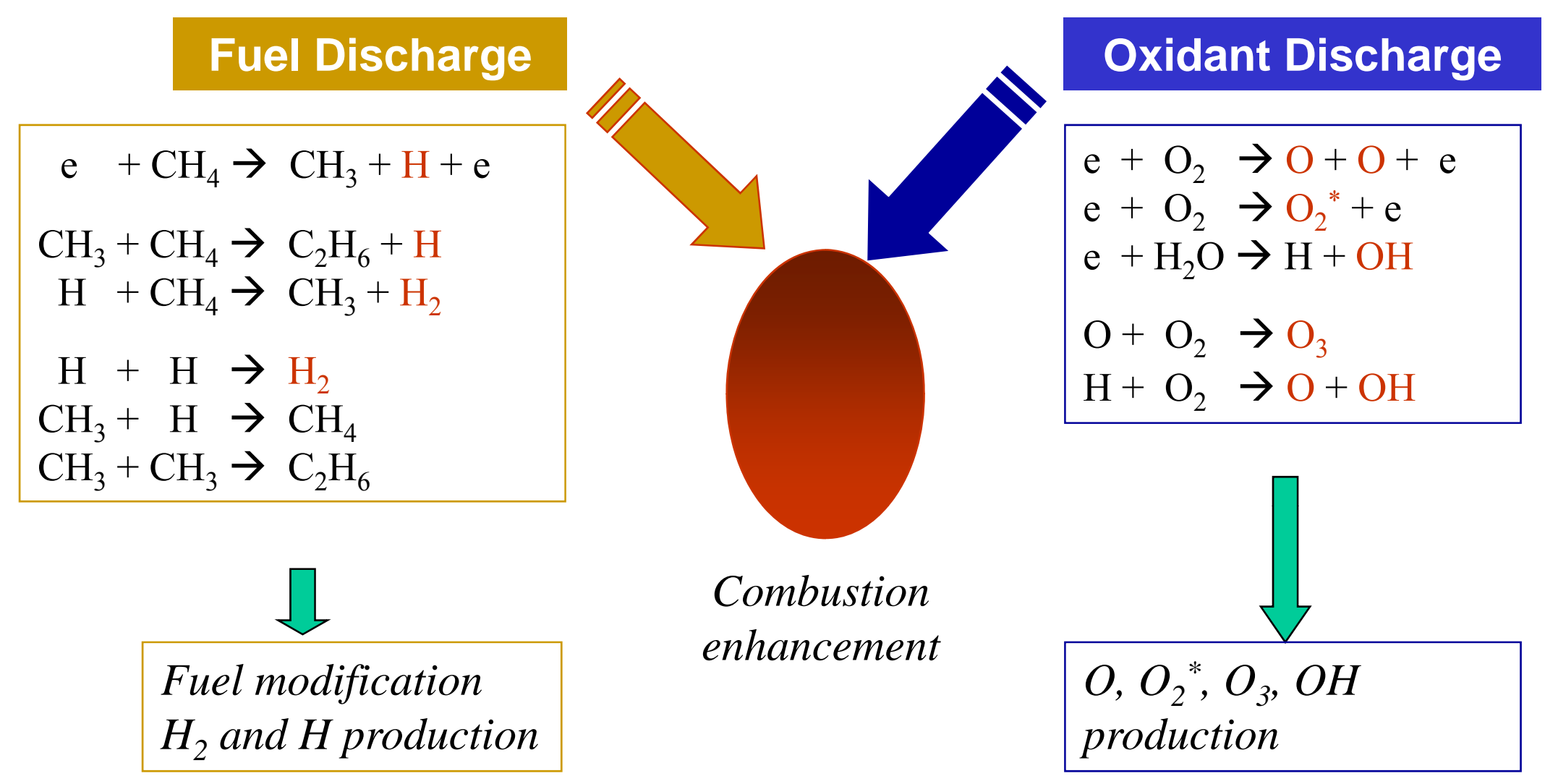

- plasma ignition inside combustion chamber

- plasma injection into combustion chamber

- plasma treatment of fuel 